Supporting Information

\title{
Cobalt-Catalyzed (E)-Selective Hydrosilylation of 1,3-Enynes for the Synthesis of 1,3-Dienylsilanes
}

\author{
Degong Kong ${ }^{\dagger, \dagger}$ Bowen $\mathrm{Hu},{ }^{\dagger}$ Min Yang ${ }^{\dagger}$ Haiping Xia, ${ }^{\star},{ }^{\dagger}$ and Dafa Chen ${ }^{*},{ }^{\dagger}$ \\ 'Shenzhen Grubbs Institute, Department of Chemistry, Southern University of Science and Technology, \\ Shenzhen 518055, People's Republic of China \\ ${ }^{\ddagger}$ MIIT Key Laboratory of Critical Materials Technology for New Energy Conversion and Storage, \\ School of Chemistry and Chemical Engineering, Harbin Institute of Technology, Harbin 150001, \\ People's Republic of China \\ Email for H. X.: xiahp@ @sustech.edu.cn; \\ Email for D. C.: chendf@ sustech.edu.cn.
}

\section{Contents}

General Procedure for the Preparation of Cobalt Complexes ................................................ S2

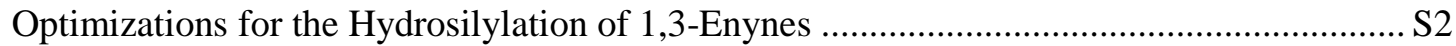

Procedures for the Deuterium-Labelling Experiments ........................................................... 4

Procedure for the Synthesis of the Cobalt(I) Hydride Species ............................................... S6

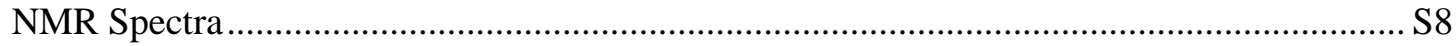




\section{General Procedure for the Preparation of Cobalt Complexes}

A $10 \mathrm{~mL}$ Schlenk flask was charged with the ligands $(0.4 \mathrm{mmol}), \mathrm{CoX}_{2}(0.4 \mathrm{mmol})$ and $5 \mathrm{~mL}$ THF under the atmosphere of nitrogen, the mixture was stirred at room temperature for $12 \mathrm{~h}$. Removing the solvent by filtration afforded powder. The cake was washed with ether and dried in vacuum to give the corresponding cobalt complexes.

\section{Optimizations for the Hydrosilylation of 1,3-Enynes}

Table S1 Optimization for the Hydrosilylation of 4-Aryl-1,3-Enynes

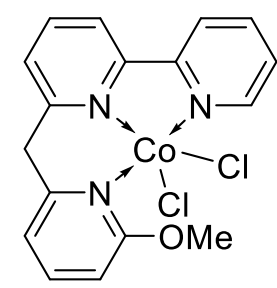

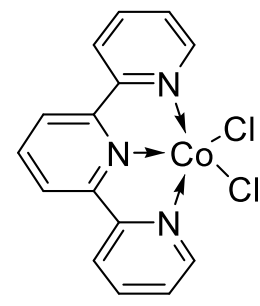<smiles>ClP1CC[Pb](Cl)(Cl)[Pb]1</smiles><smiles>P[Pb]1CCC[Pb](Cl)[Pb]1Cl</smiles><smiles>PP1(Cl)(Cl)CCCC[Pb]1(Cl)[Pb]</smiles><smiles>[Te]C=[Te]</smiles>

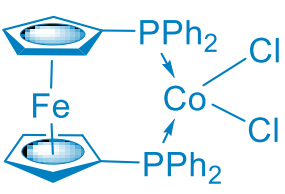

Dppf-CoCl 2 $\mathrm{L}_{2}-\mathrm{CoCl}_{2}$<smiles>[2H][Pb](=O)[O-]</smiles>

Dppp-CoCl 2

Dppb-CoCl

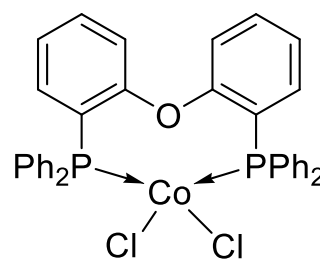<smiles>CC1(C)c2cccc(P)c2Oc2c(P(O)c3ccccc3)cccc21</smiles>

Xantphos-CoCl 2

Dppf-CoBr

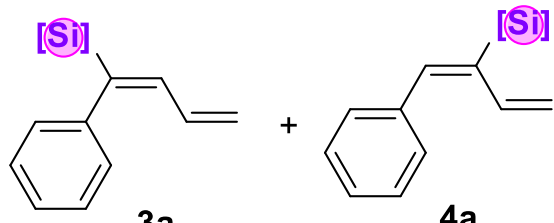

$3 a$

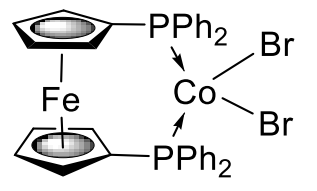

(Si)

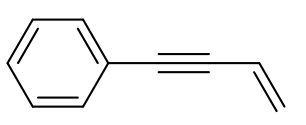

$1 a$
2

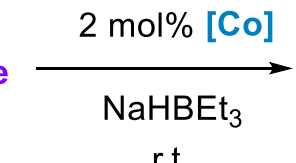

\begin{tabular}{|c|c|c|c|c|c|c|}
\hline Entry & Cat. & Silane & Additive & Solvent & 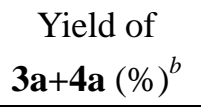 & $\begin{array}{c}\text { Ratio of } \\
\mathbf{3 a}: \mathbf{4 a}^{b}\end{array}$ \\
\hline 1 & $\mathrm{~L}_{1}-\mathrm{CoCl}_{2}$ & $\mathrm{PhSiH}_{3}$ & $\mathrm{NaHBEt}_{3}$ & Toluene & 61 & $86: 14$ \\
\hline 2 & $\mathrm{~L}_{2}-\mathrm{CoCl}_{2}$ & $\mathrm{PhSiH}_{3}$ & $\mathrm{NaHBEt}_{3}$ & Toluene & trace & -- \\
\hline 3 & Dppe-CoCl${ }_{2}$ & $\mathrm{PhSiH}_{3}$ & $\mathrm{NaHBEt}_{3}$ & Toluene & $<10$ & -- \\
\hline 4 & Dppp-CoCl 2 & $\mathrm{PhSiH}_{3}$ & $\mathrm{NaHBEt}_{3}$ & Toluene & 21 & $80: 20$ \\
\hline 5 & $\mathrm{Dppb}-\mathrm{CoCl}_{2}$ & $\mathrm{PhSiH}_{3}$ & $\mathrm{NaHBEt}_{3}$ & Toluene & 27 & $79: 21$ \\
\hline 6 & Dppf-CoCl${ }_{2}$ & $\mathrm{PhSiH}_{3}$ & $\mathrm{NaHBEt}_{3}$ & Toluene & 91 & $97: 3$ \\
\hline 7 & Dppf-CoBr 2 & $\mathrm{PhSiH}_{3}$ & $\mathrm{NaHBEt}_{3}$ & Toluene & 89 & $95: 5$ \\
\hline 8 & Dpephos- $\mathrm{CoCl}_{2}$ & $\mathrm{PhSiH}_{3}$ & $\mathrm{NaHBEt}_{3}$ & Toluene & 88 & $94: 6$ \\
\hline 9 & Xantphos- $\mathrm{CoCl}_{2}$ & $\mathrm{PhSiH}_{3}$ & $\mathrm{NaHBEt}_{3}$ & Toluene & 71 & $92: 8$ \\
\hline
\end{tabular}




\begin{tabular}{|c|c|c|c|c|c|c|}
\hline 10 & Dppf-CoCl ${ }_{2}$ & $\mathrm{PhSiH}_{3}$ & $\mathrm{NaHBEt}_{3}$ & n-hexane & 90 & $97: 3$ \\
\hline 11 & Dppf-CoCl ${ }_{2}$ & $\mathrm{PhSiH}_{3}$ & $\mathrm{NaHBEt}_{3}$ & THF & 88 & $95: 5$ \\
\hline 12 & Dppf-CoCl ${ }_{2}$ & $\mathrm{PhSiH}_{3}$ & $\mathrm{NaHBEt}_{3}$ & $\mathrm{Et}_{2} \mathrm{O}$ & 83 & $96: 4$ \\
\hline 13 & Dppf-CoCl ${ }_{2}$ & $\mathrm{PhSiH}_{3}$ & $\mathrm{NaHBEt}_{3}$ & $\mathrm{CH}_{2} \mathrm{Cl}_{2}$ & 47 & $93: 7$ \\
\hline 14 & Dppf-CoCl ${ }_{2}$ & $\mathrm{Ph}_{2} \mathrm{SiH}_{2}$ & $\mathrm{NaHBEt}_{3}$ & Toluene & 90 & $94: 6$ \\
\hline 15 & Dppf-CoCl ${ }_{2}$ & $\mathrm{Et}_{3} \mathrm{SiH}$ & $\mathrm{NaHBEt}_{3}$ & Toluene & n.r. ${ }^{c}$ & -- \\
\hline 16 & Dppf-CoCl ${ }_{2}$ & $\mathrm{Ph}_{3} \mathrm{SiH}$ & $\mathrm{NaHBEt}_{3}$ & Toluene & trace & -- \\
\hline 17 & Dppf-CoCl ${ }_{2}$ & $\mathrm{PhSiH}_{3}$ & $\mathrm{KN}\left(\mathrm{SiCH}_{3}\right)_{2}$ & Toluene & 23 & $96: 4$ \\
\hline 18 & Dppf-CoCl ${ }_{2}$ & $\mathrm{PhSiH}_{3}$ & $\mathrm{NaO}^{t} \mathrm{Bu}$ & Toluene & 83 & $97: 3$ \\
\hline 19 & $\mathrm{Co}(\mathrm{acac})_{2} / \mathrm{Dppf}$ & $\mathrm{PhSiH}_{3}$ & -- & Toluene & 13 & -- \\
\hline 20 & $\begin{array}{l}\mathrm{Co}(\mathrm{acac})_{2} / \\
\text { Xantphos }\end{array}$ & $\mathrm{PhSiH}_{3}$ & -- & Toluene & 15 & -- \\
\hline
\end{tabular}

${ }^{a}$ Reaction conditions: But-3-en-1-yn-1-ylbenzene $(0.5 \mathrm{mmol})$, silane $(0.5 \mathrm{mmol})$, [Co] (2 mol\%), $\mathrm{NaHBEt}_{3}(6 \mathrm{~mol} \%)$, solvent $(2 \mathrm{~mL})$, room temperature, $1 \mathrm{~h} .{ }^{b}$ Yield and ratio were determined by ${ }^{1} \mathrm{H}$ NMR using 1,3,5-methoxybenzene as an internal standard. ${ }^{c}$ n.r. $=$ No reaction.

Table S2 Optimization for the Hydrosilylation of 4-Alkyl-1,3-Enynes

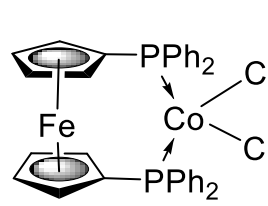

Dppf-CoCl 2<smiles>Pc1ccccc1Oc1ccccc1P(c1ccccc1)[Ge](Cl)(Cl)Cl</smiles>

DPEphos- $\mathrm{CoCl}_{2}$<smiles>CC1(C)c2cccc(P)c2Oc2c1cccc2P(Cl)(Cl)(Cl)Cl</smiles>

Xantphos- $\mathrm{CoCl}_{2}$

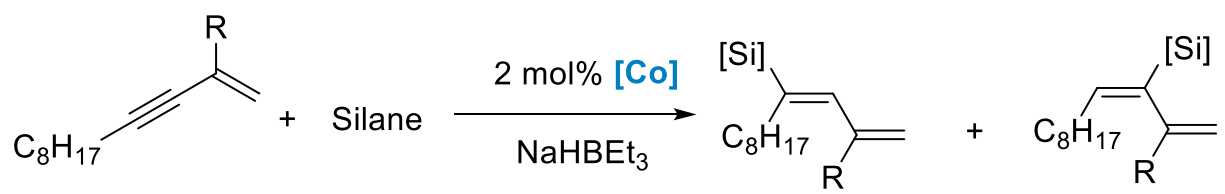

1t: $\mathrm{R}=\mathrm{H}$

1aa: $R=M e$
2

(20)

3t: $\mathrm{R}=\mathrm{H},[\mathrm{Si}]=\mathrm{SiH}_{2} \mathrm{Ph}$ 3aa: $\mathrm{R}=\mathrm{Me},[\mathrm{Si}]=\mathrm{SiH}_{2} \mathrm{Ph}$ 3t': $\mathrm{R}=\mathrm{H}$, $[\mathrm{Si}]=\mathrm{SiHPh}_{2}$ 3aa': $\mathrm{R}=\mathrm{Me}$, $[\mathrm{Si}]=\mathrm{SiHPh}_{2}$ 4t: $\mathrm{R}=\mathrm{H}$, $[\mathrm{Si}]=\mathrm{SiH}_{2} \mathrm{Ph}$

4aa: $\mathrm{R}=\mathrm{Me},[\mathrm{Si}]=\mathrm{SiH}_{2} \mathrm{Ph}$

4t': $\mathrm{R}=\mathrm{H}$, [Si] $=\mathrm{SiHPh}_{2}$

4aa': $\mathrm{R}=\mathrm{Me},[\mathrm{Si}]=\mathrm{SiHPh}_{2}$

\begin{tabular}{cccccccc}
\hline Entry & Cat. & Solvent & Silane & $\begin{array}{c}\text { Enyne/ } \\
\text { Silane }\end{array}$ & $\mathrm{R}$ & $\begin{array}{c}\text { Yield of } \\
\mathbf{3 + 4}(\%)^{b}\end{array}$ & $\begin{array}{c}\text { Ratio of } \\
\mathbf{3} \mathbf{4}^{b}\end{array}$ \\
1 & Dppf-CoCl & Toluene & $\mathrm{PhSiH}_{3}$ & $1: 1$ & $\mathrm{H}$ & 88 & $47: 53$ \\
2 & $\mathrm{DPEphos}-\mathrm{CoCl}_{2}$ & Toluene & $\mathrm{PhSiH}_{3}$ & $1: 1$ & $\mathrm{H}$ & 80 & $45: 55$ \\
3 & $\mathrm{Xantphos-CoCl}_{2}$ & Toluene & $\mathrm{PhSiH}_{3}$ & $1: 1$ & $\mathrm{H}$ & 91 & $29: 71$ \\
4 & $\mathrm{Xantphos-CoCl}_{2}$ & Toluene & $\mathrm{Ph}_{2} \mathrm{SiH}_{2}$ & $1: 1$ & $\mathrm{H}$ & 93 & $31: 69$ \\
5 & $\mathrm{Xantphos-CoCl}_{2}$ & Toluene & $\mathrm{Ph}_{2} \mathrm{SiH}_{2}$ & $1: 1.5$ & $\mathrm{H}$ & 91 & $28: 72$ \\
6 & $\mathrm{Xantphos-CoCl}_{2}$ & Toluene & $\mathrm{Ph}_{2} \mathrm{SiH}_{2}$ & $1: 2$ & $\mathrm{H}$ & 86 & $21: 79$ \\
$7^{c}$ & $\mathrm{Xantphos-CoCl}_{2}$ & -- & $\mathrm{Ph}_{2} \mathrm{SiH}_{2}$ & $1: 1$ & $\mathrm{H}$ & 87 & $25: 75$ \\
\hline
\end{tabular}




\begin{tabular}{cccccccc}
\hline $8^{c}$ & Xantphos-CoCl & -- & $\mathrm{Ph}_{2} \mathrm{SiH}_{2}$ & $1: 2$ & $\mathrm{H}$ & 58 & $4: 96$ \\
$9^{c}$ & Xantphos-CoCl & -- & $\mathrm{PhSiH}_{3}$ & $1: 1$ & $\mathrm{H}$ & 77 & $22: 78$ \\
$10^{c}$ & Xantphos-CoCl & -- & $\mathrm{PhSiH}_{3}$ & $1: 2$ & $\mathrm{H}$ & 52 & $6: 94$ \\
$11^{c}$ & Dppf-CoCl & -- & $\mathrm{PhSiH}_{3}$ & $1: 2$ & $\mathrm{H}$ & 48 & $48: 52$ \\
12 & Xantphos-CoCl & Toluene & $\mathrm{PhSiH}_{3}$ & $1: 1$ & $\mathrm{Me}$ & 91 & $3: 97$ \\
13 & Xantphos-CoCl & Toluene & $\mathrm{Ph}_{2} \mathrm{SiH}_{2}$ & $1: 1$ & $\mathrm{Me}$ & 90 & $6: 94$ \\
\hline
\end{tabular}

${ }^{a}$ Reaction conditions: 4-Alkyl-1,3-enyne $(0.25 \mathrm{mmol})$, silane $(0.25 \mathrm{mmol}),[\mathrm{Co}](2 \mathrm{~mol} \%)$, $\mathrm{NaHBEt}_{3}\left(6 \mathrm{~mol} \%, 1 \mathrm{~mol} / \mathrm{L}\right.$ in THF), room temperature, $1 \mathrm{~h} .{ }^{b}$ Yield and ratio were determined by ${ }^{1} \mathrm{H}$ NMR using 1,3,5-methoxybenzene as an internal standard. ${ }^{c}$ Solvent-free.

\section{Procedures for the Deuterium-Labelling Experiments}

(A)

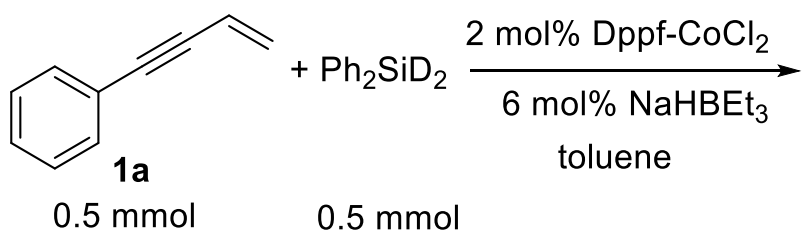

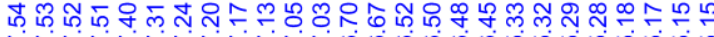

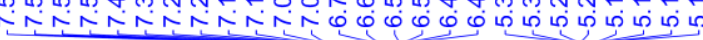

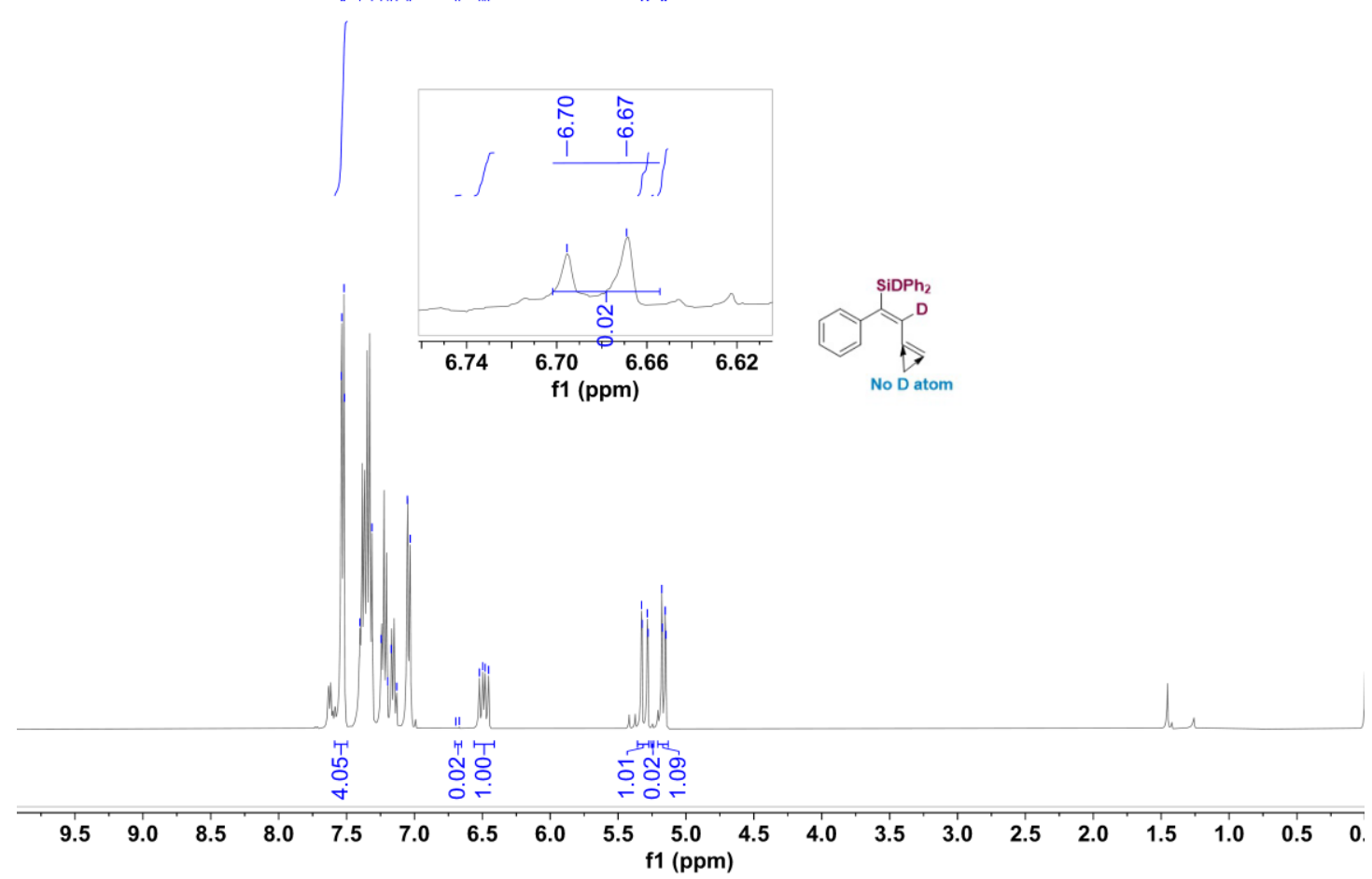

Figure S1 ${ }^{1} \mathrm{H}$ NMR $\left(\mathrm{CDCl}_{3}, 400 \mathrm{M}\right)$ spectrum of 3a'-D (Relaxation delay D1 = 25

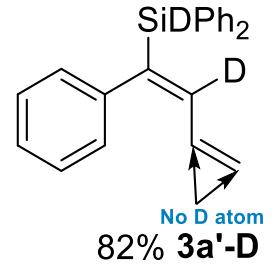

$82 \% 3 a '-D$ 
In a nitrogen filled glovebox, the cobalt complex Dppf- $\mathrm{CoCl}_{2}(6.8 \mathrm{mg}, 2 \mathrm{~mol} \%)$ and anhydrous toluene $(2 \mathrm{~mL})$ were added into a $20 \mathrm{~mL}$ Schlenk tube with a magnetic stirring bar. The mixture was injected with $\mathrm{NaHBEt}_{3}(1.0 \mathrm{M})(30 \mu \mathrm{L}, 0.03 \mathrm{mmol})$ by dropwise, and then the mixture of $\mathrm{Ph}_{2} \mathrm{SiD}_{2}(0.5 \mathrm{mmol})$ and 1,3 -enyne $(0.5 \mathrm{mmol})$ was added to the Schlenk tube. After 1 hour, the resulting solution was concentrated in vacuum and the crude product was purified directly by silica gel column chromatography eluting with petroleum ether to afford the corresponding product.

(B)
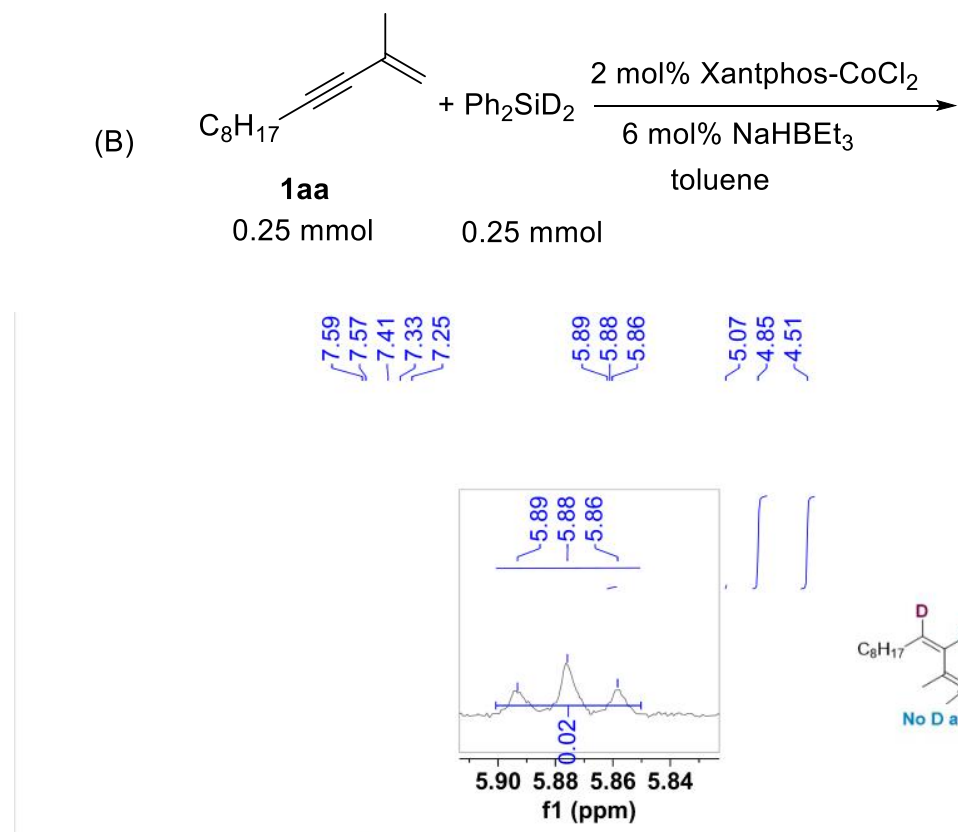
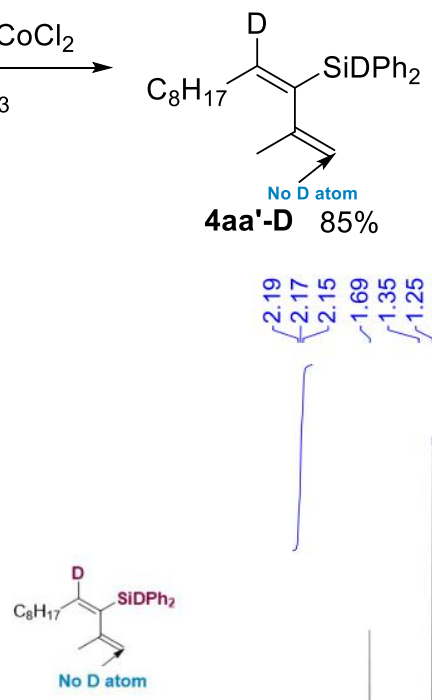

4aa'-D $85 \%$

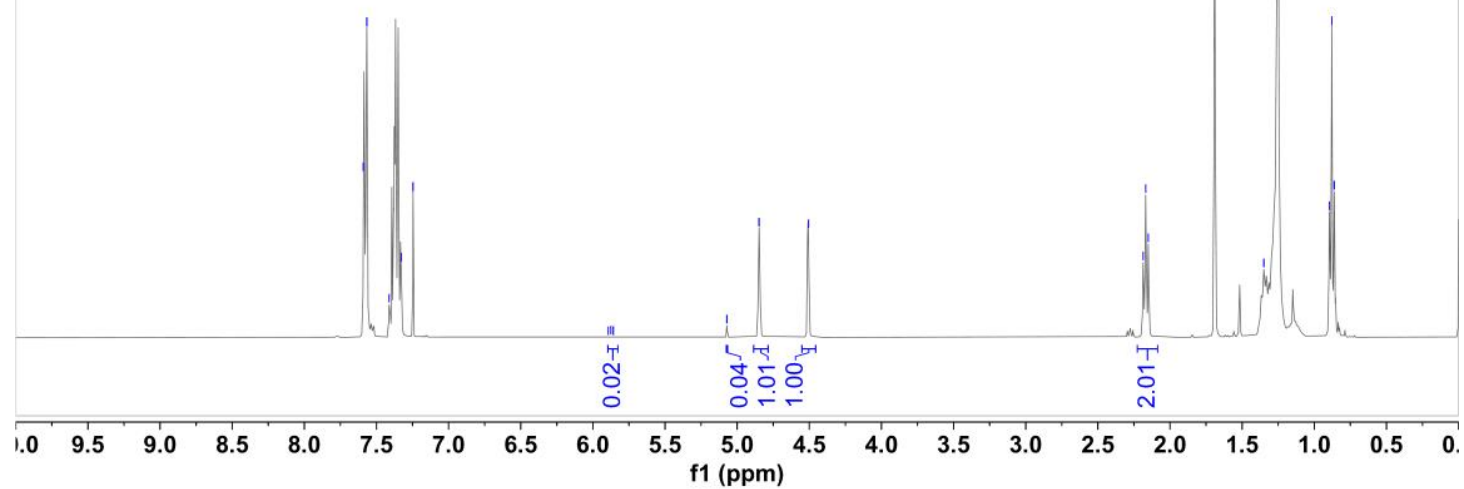

Figure S2 ${ }^{1} \mathrm{H}$ NMR $\left(\mathrm{CDCl}_{3}, \mathbf{4 0 0 M}\right)$ spectrum of 4aa'-D (Relaxation delay D1 = 25 s).

In a nitrogen filled glovebox, the cobalt complex Xantphos- $\mathrm{CoCl}_{2}(3.5 \mathrm{mg}, 2 \mathrm{~mol} \%)$ and anhydrous toluene $(1 \mathrm{~mL})$ were added into a $10 \mathrm{~mL}$ Schlenk tube with a magnetic stirring bar. The mixture was injected with $\mathrm{NaHBEt}_{3}(1.0 \mathrm{M})(15 \mu \mathrm{L}, 0.015 \mathrm{mmol})$ by dropwise, and then the mixture of $\mathrm{Ph}_{2} \mathrm{SiD}_{2}(0.25 \mathrm{mmol})$ and alkyl-1,3-enyne (1aa) $(0.25 \mathrm{mmol})$ was added to the Schlenk tube. After 1 hour, the resulting solution was concentrated in vacuum and the crude product was purified directly by silica gel column chromatography eluting with petroleum ether to afford the corresponding product. 


\section{Procedure for the Synthesis of the Cobalt(I) Hydride Species}

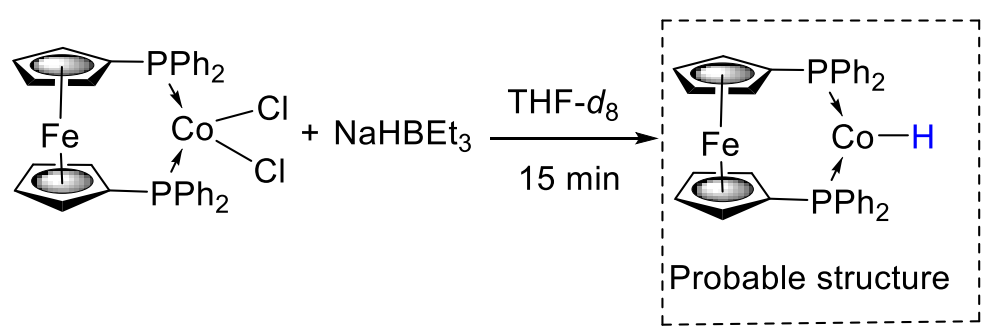

Figure S3 Synthesis of the Cobalt(I) Hydride Species

In a nitrogen filled glovebox, the cobalt complex Dppf-CoCl $2(6.8 \mathrm{mg}, 0.01 \mathrm{mmol})$ and $0.6 \mathrm{~mL}$ THF- $d_{8}$ were added into an NMR tube equipped with Teflon valve at $-20{ }^{\circ} \mathrm{C}$, the mixture was injected with $0.03 \mathrm{mmol} \mathrm{NaHBEt}_{3}(30 \mathrm{uL}, 1 \mathrm{~mol} / \mathrm{L}$ in THF). After $15 \mathrm{~min}$, the tube was placed to an NMR machine to analyze ${ }^{1} \mathrm{H}$ and ${ }^{31} \mathrm{P}$ NMR data ( $\delta \mathrm{Co}-\mathrm{H}=-11.89 \mathrm{ppm}, \delta \mathrm{P}=60.96 \mathrm{ppm}$ ).

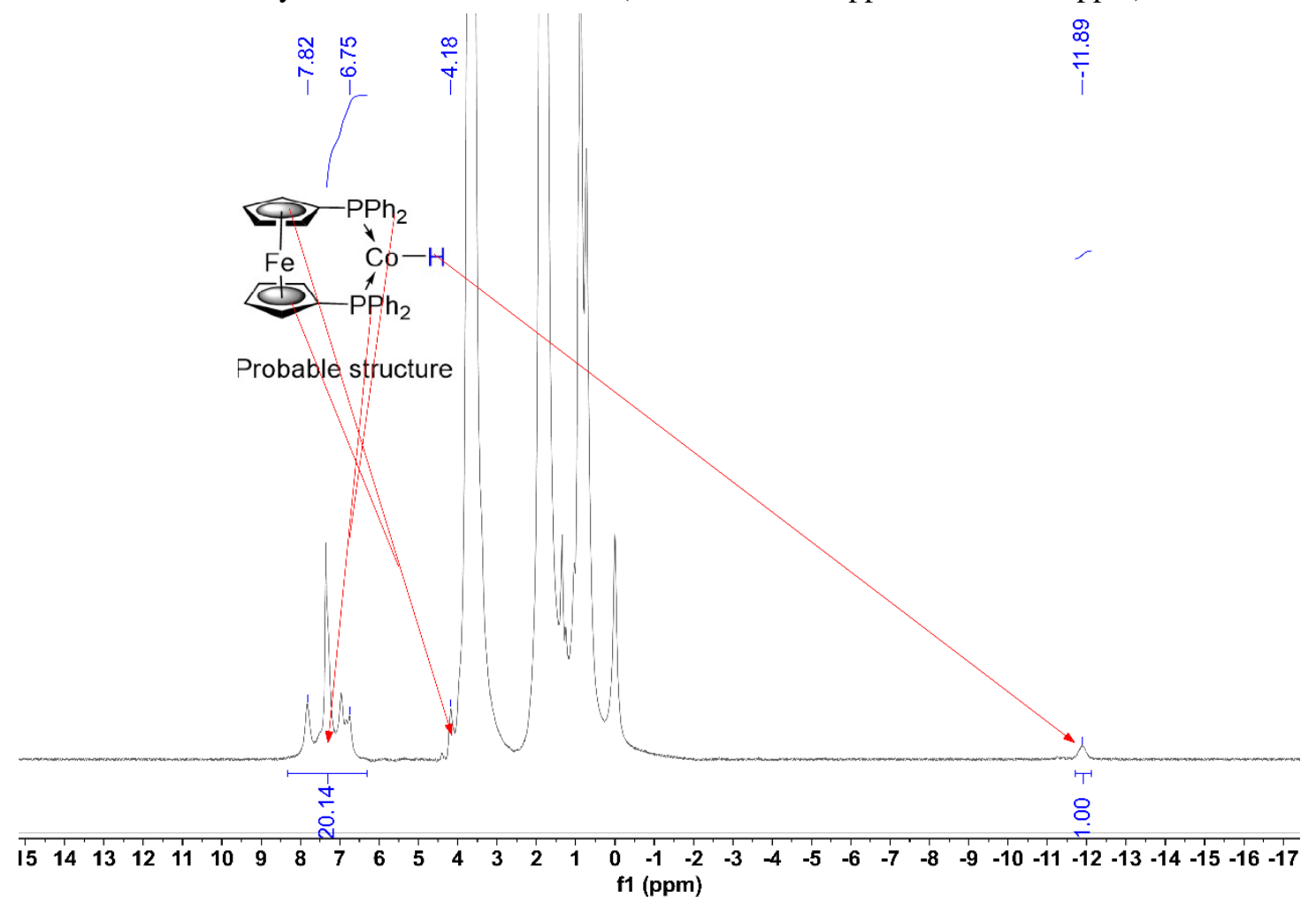

Figure S4 ${ }^{1} \mathrm{H}$ NMR (THF- $\left.d_{8}, 600 \mathrm{M}\right)$ spectrum of the Cobalt(I) Hydride Species 


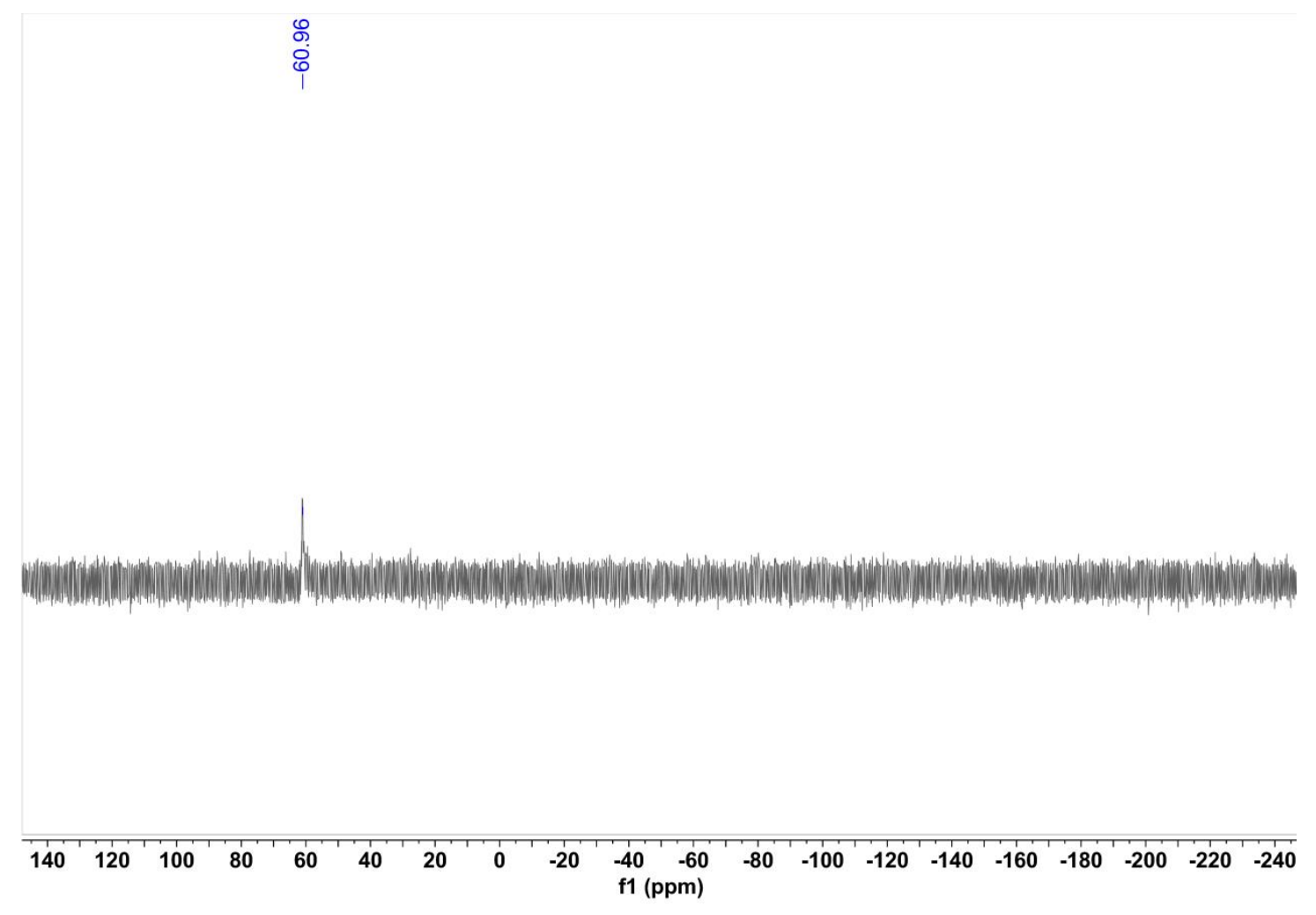

Figure $S 5{ }^{31}$ P NMR (THF- $\left.d_{8}, 243 M\right)$ spectrum of the Cobalt(I) Hydride Species 
NMR Spectra

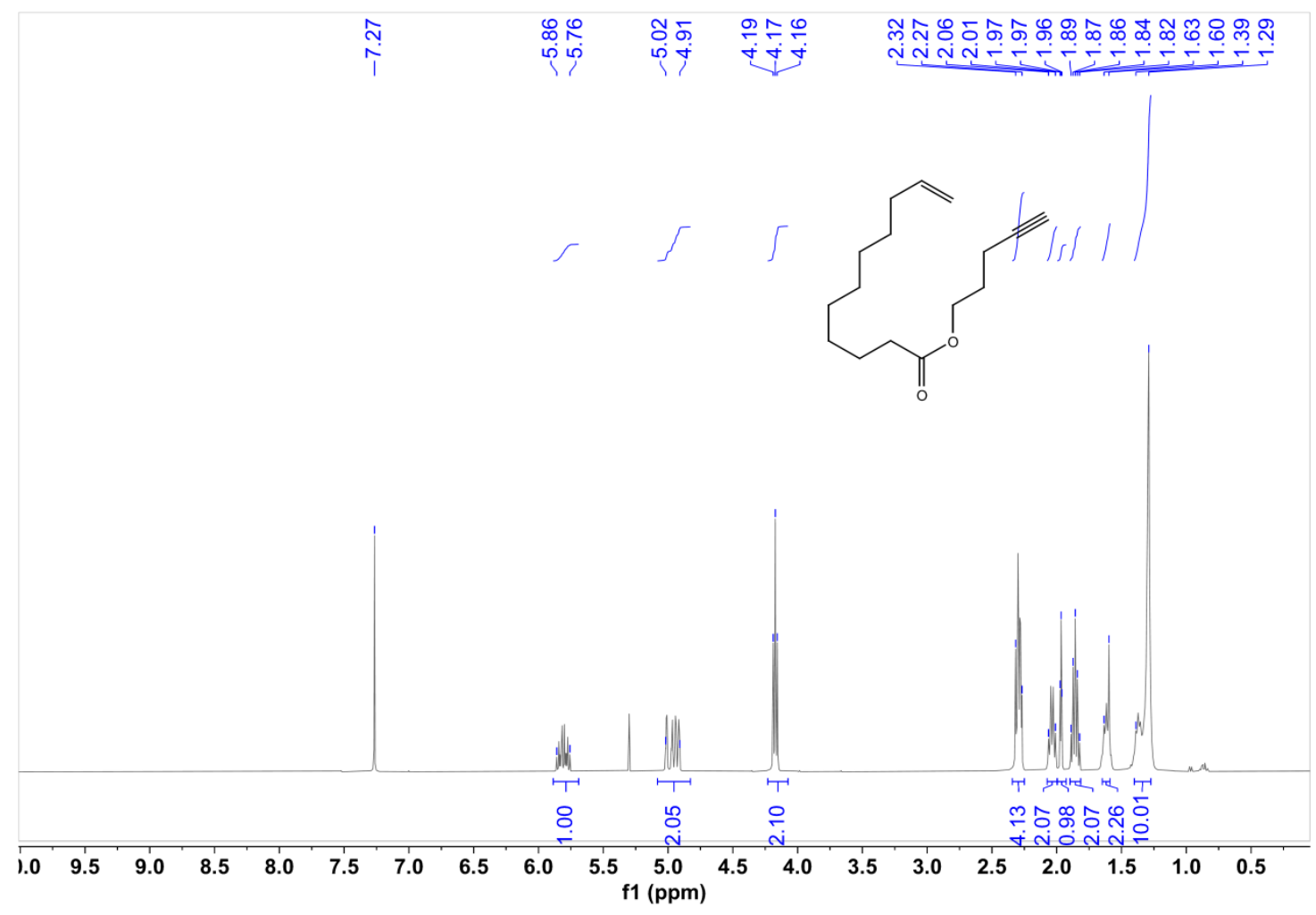

Figure S6 ${ }^{1} \mathrm{H}$ NMR $\left(\mathrm{CDCl}_{3}, 400 \mathrm{M}\right)$ spectrum of S-1ah

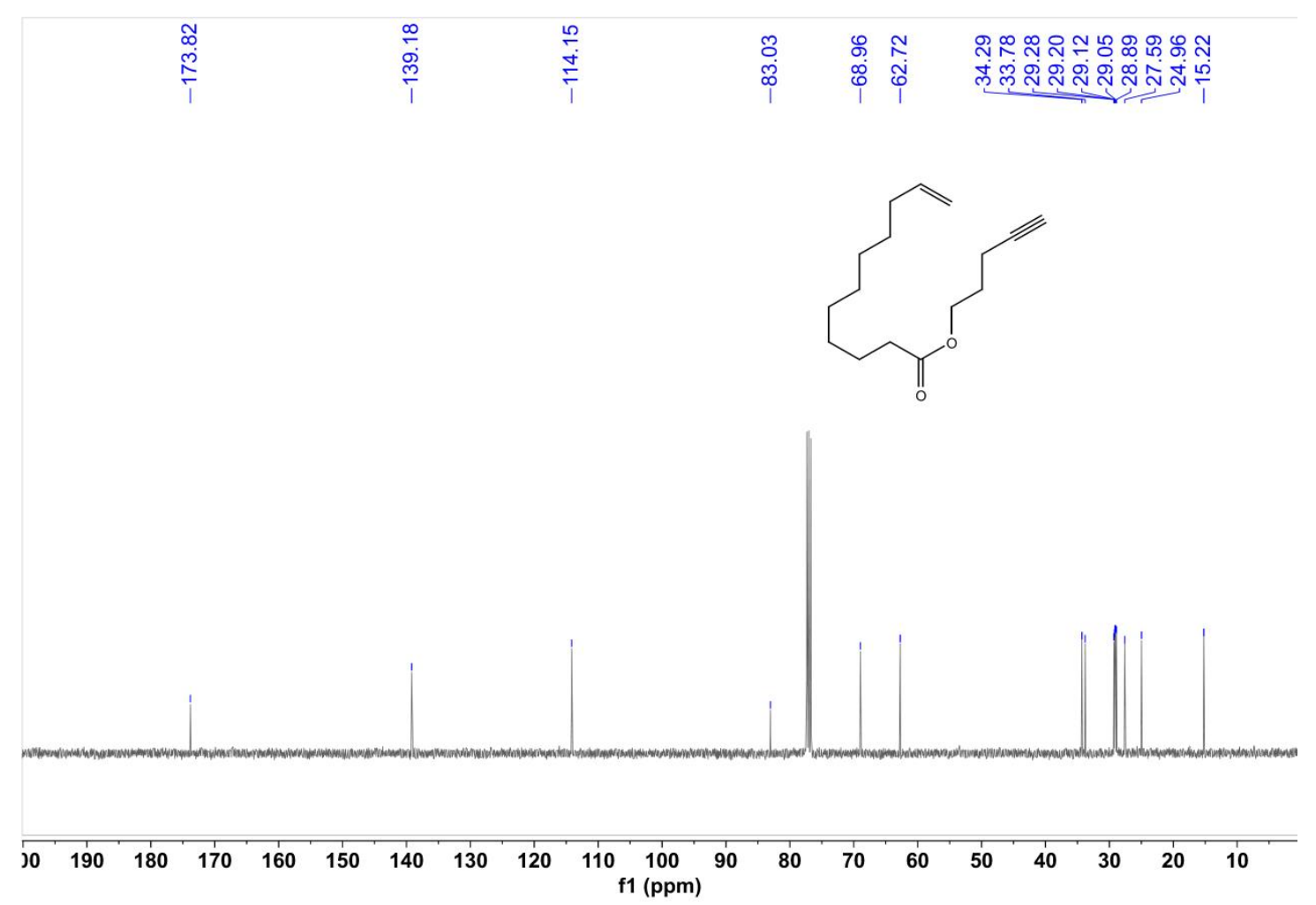

Figure $\mathrm{S} 7{ }^{13} \mathrm{C} \mathrm{NMR}\left(\mathrm{CDCl}_{3}, 100 \mathrm{M}\right)$ spectrum of S-1ah 


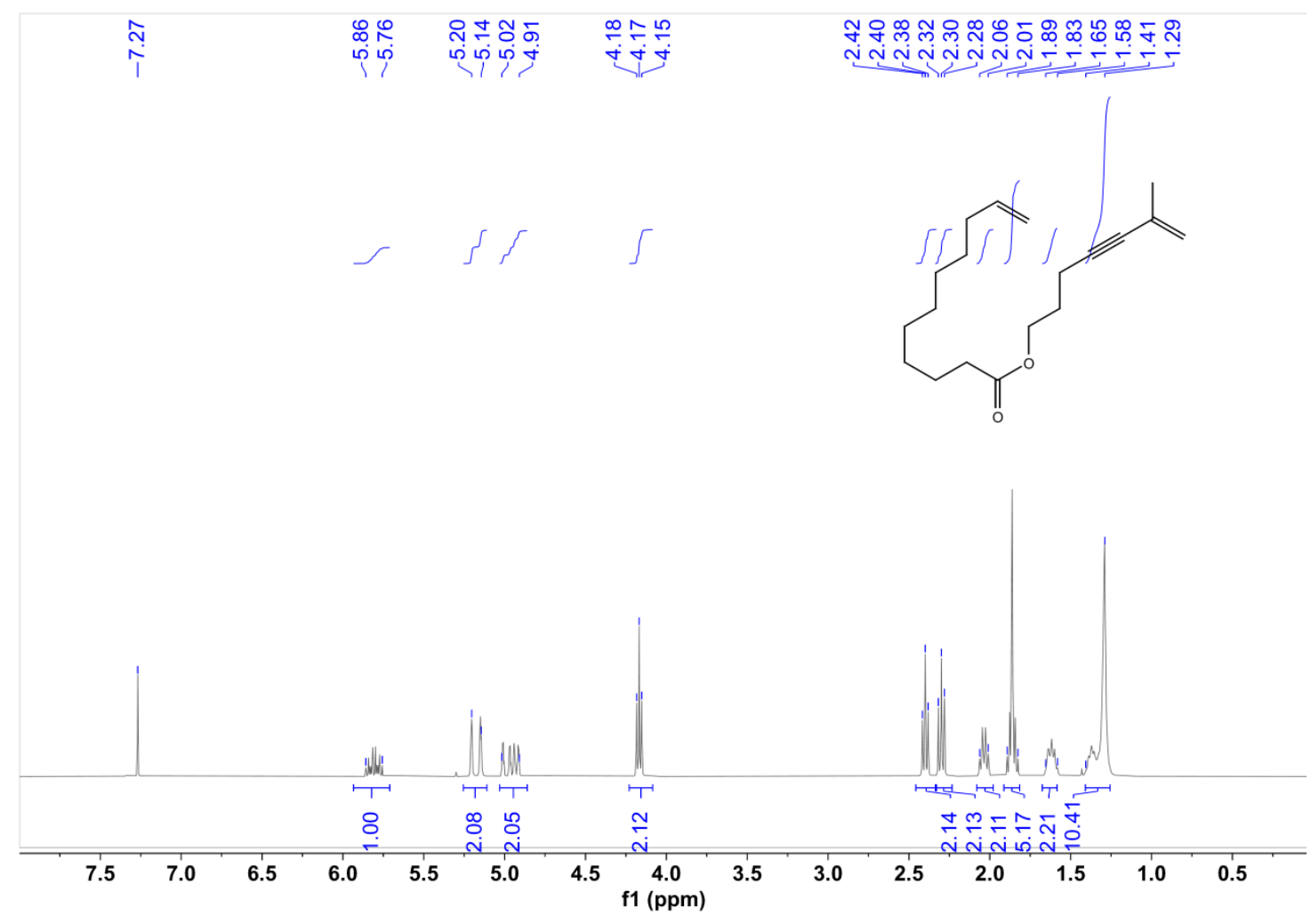

Figure S8 ${ }^{1} \mathrm{H}$ NMR $\left(\mathrm{CDCl}_{3}, 400 \mathrm{M}\right)$ spectrum of $1 \mathrm{ah}$

\begin{tabular}{|c|c|c|c|c|c|}
\hline & $\stackrel{\infty}{\frac{\infty}{\sigma}}$ & 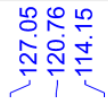 & $\begin{array}{ll}\infty & \stackrel{g}{+} \\
\substack{0 \\
\infty} & \infty \\
1 & 1\end{array}$ & $\begin{array}{l}\stackrel{2}{\mathrm{~S}} \\
\text { త్ర } \\
1\end{array}$ & 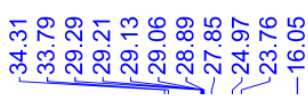 \\
\hline
\end{tabular}
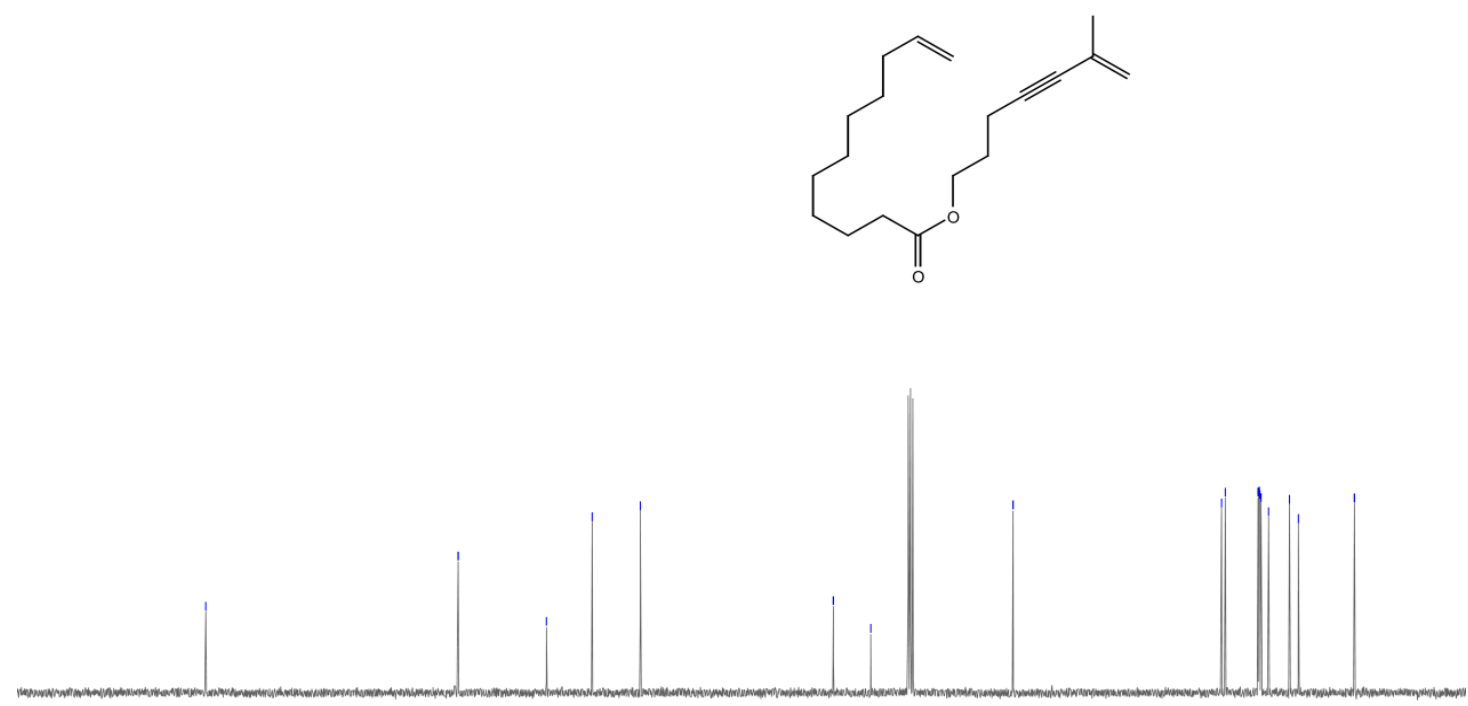

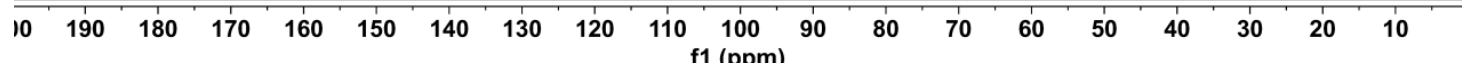

Figure S9 ${ }^{13} \mathrm{C}$ NMR $\left(\mathrm{CDCl}_{3}, 100 \mathrm{M}\right)$ spectrum of 1 ah 


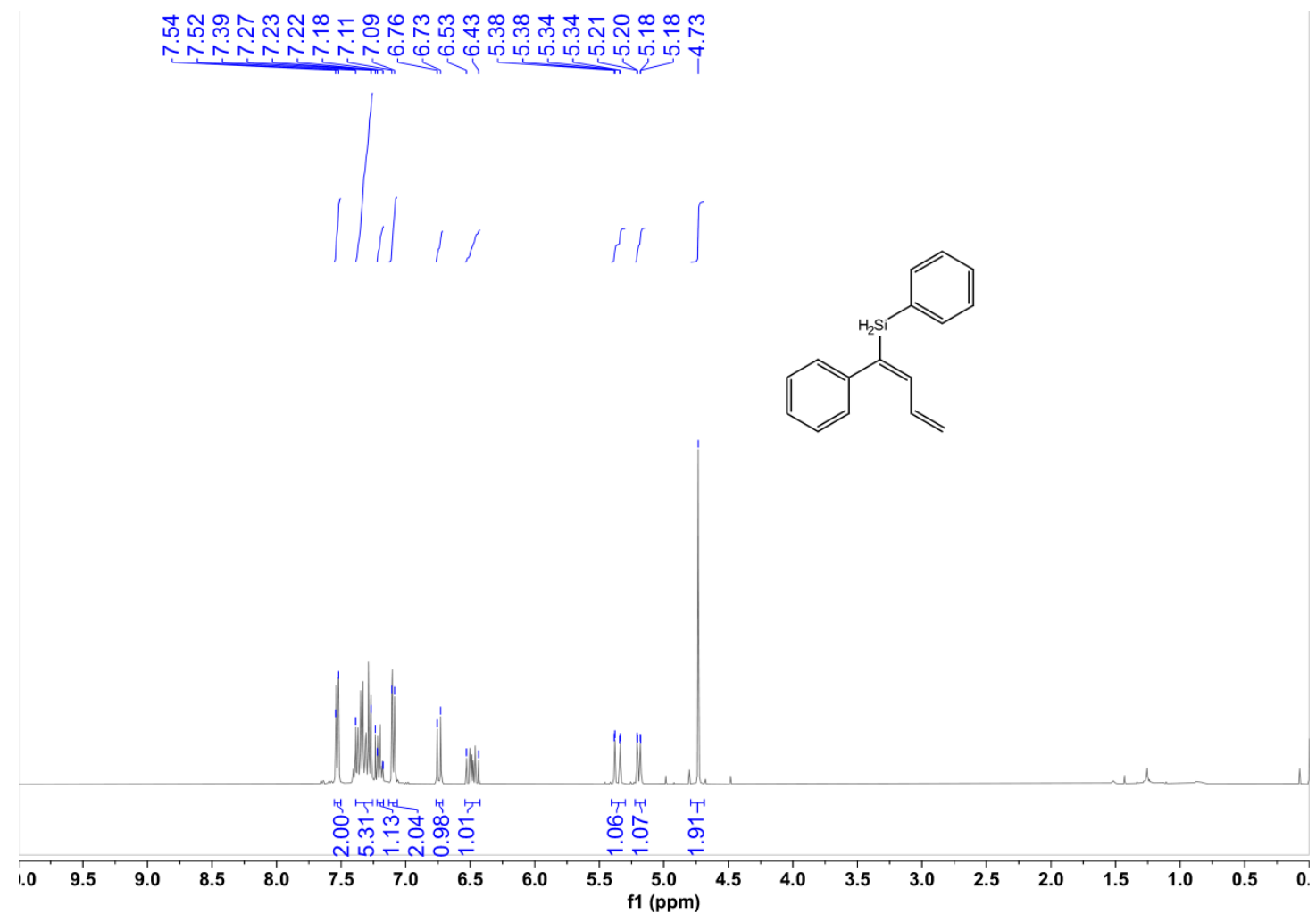

Figure $\mathrm{S10}^{1} \mathrm{H} \mathrm{NMR}\left(\mathrm{CDCl}_{3}, 400 \mathrm{M}\right)$ spectrum of $3 \mathrm{a}$

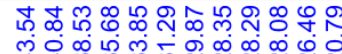

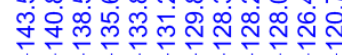

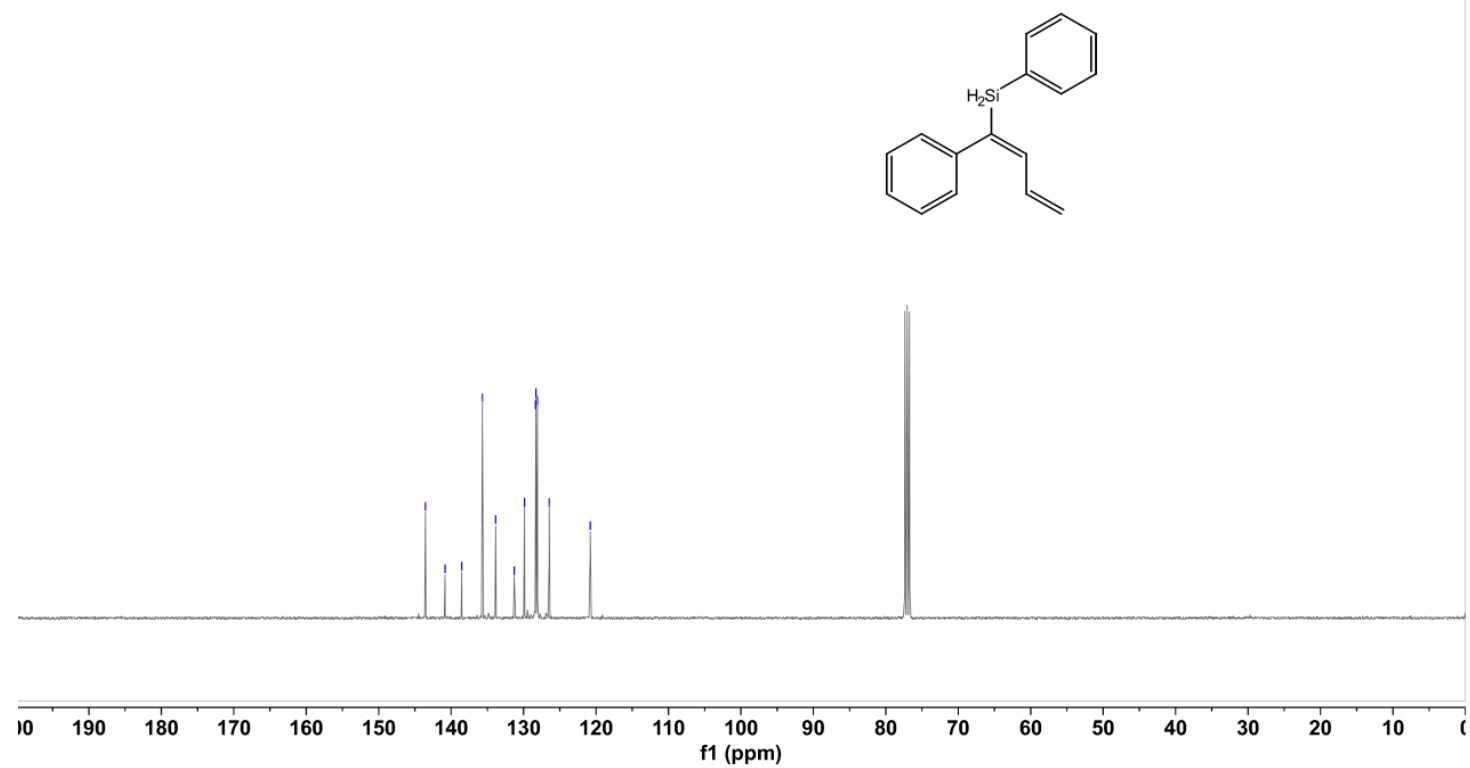

Figure $\mathrm{S}_{11}{ }^{13} \mathrm{C} \mathrm{NMR}\left(\mathrm{CDCl}_{3}, 100 \mathrm{M}\right)$ spectrum of 3a 


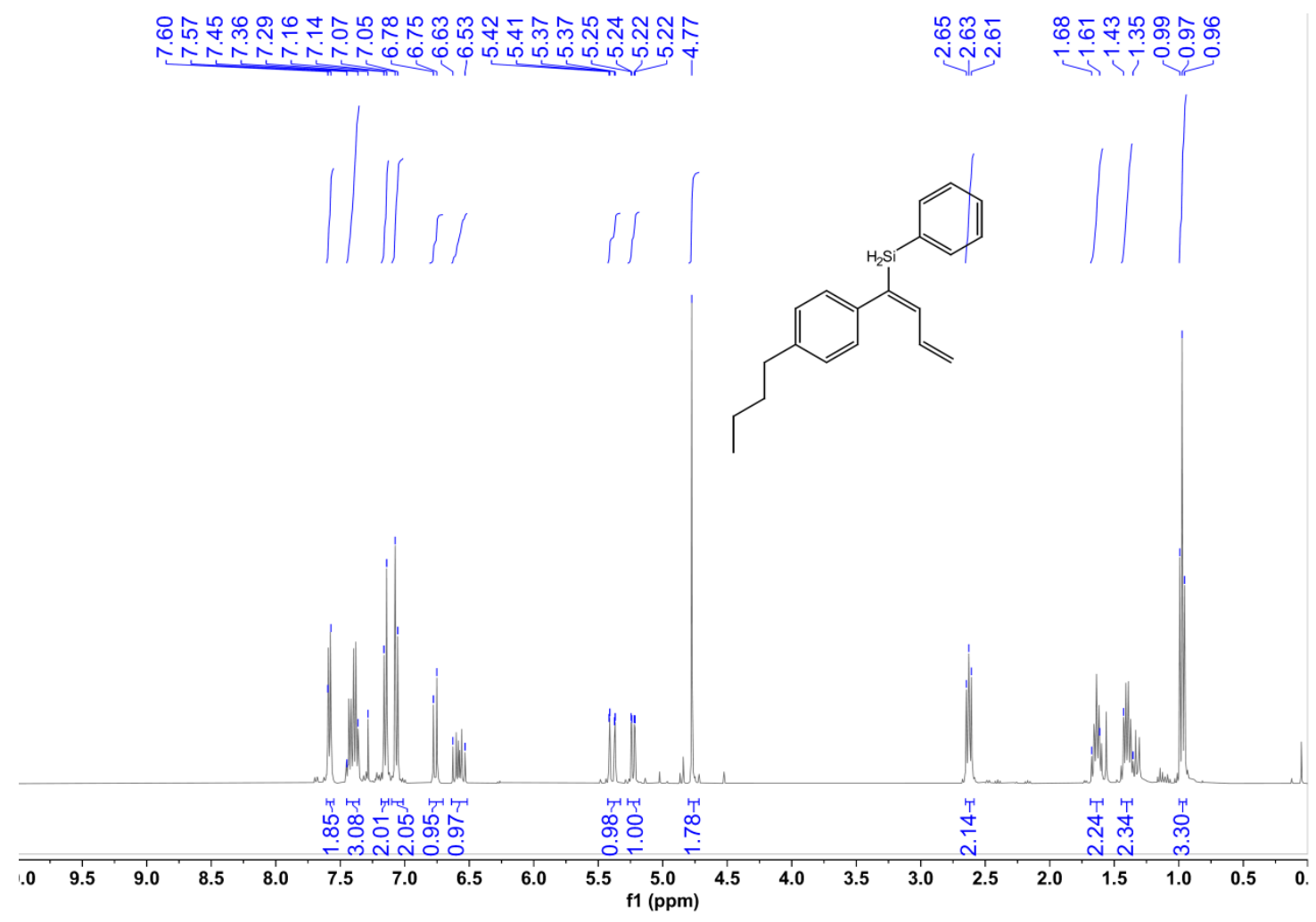

Figure $\mathrm{S12}^{1} \mathrm{H} \mathrm{NMR}\left(\mathrm{CDCl}_{3}, 400 \mathrm{M}\right)$ spectrum of $3 \mathrm{~b}$

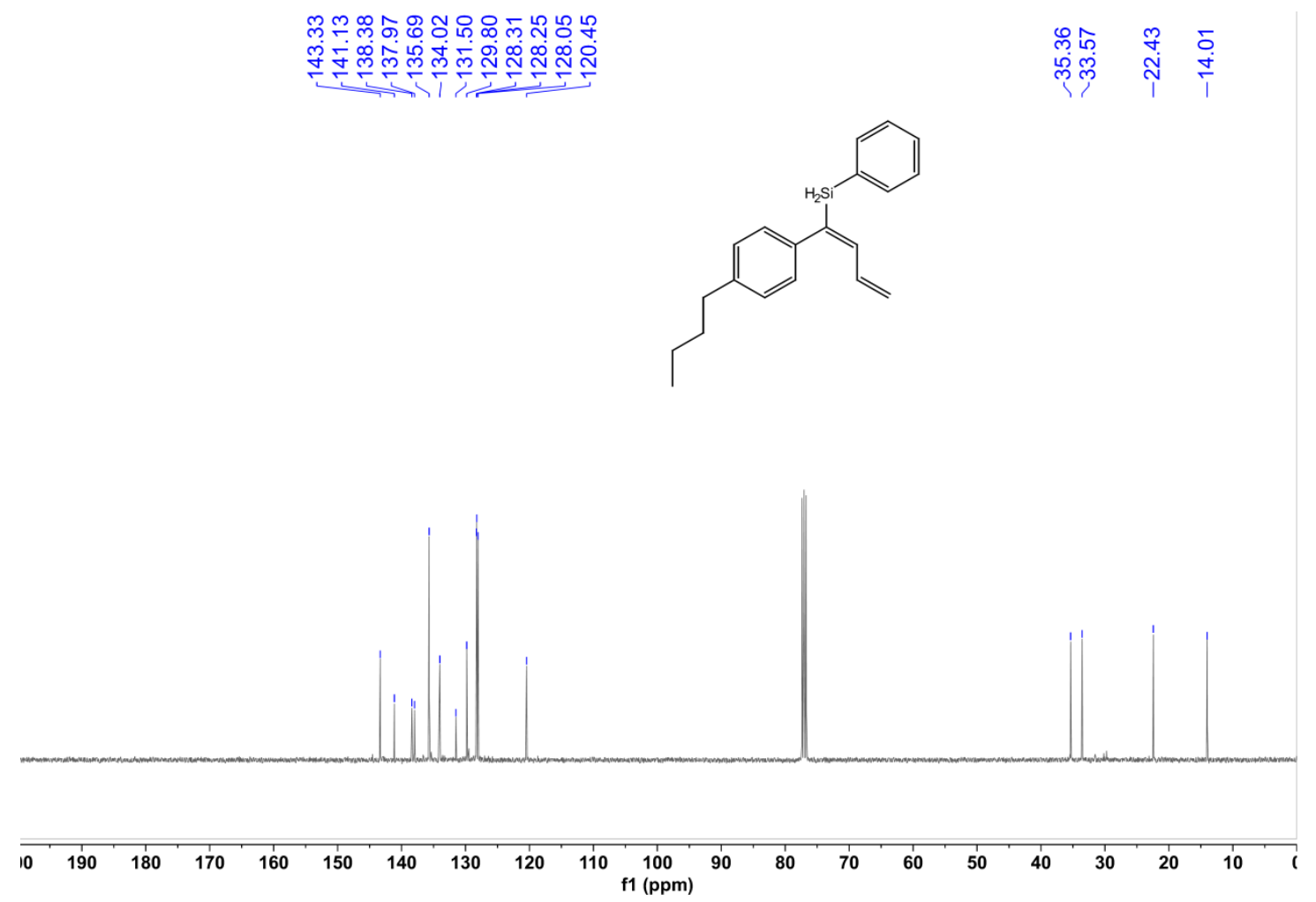

Figure $\mathrm{S}^{13}{ }^{13} \mathrm{C} \mathrm{NMR}\left(\mathrm{CDCl}_{3}, 100 \mathrm{M}\right)$ spectrum of $3 \mathrm{~b}$ 

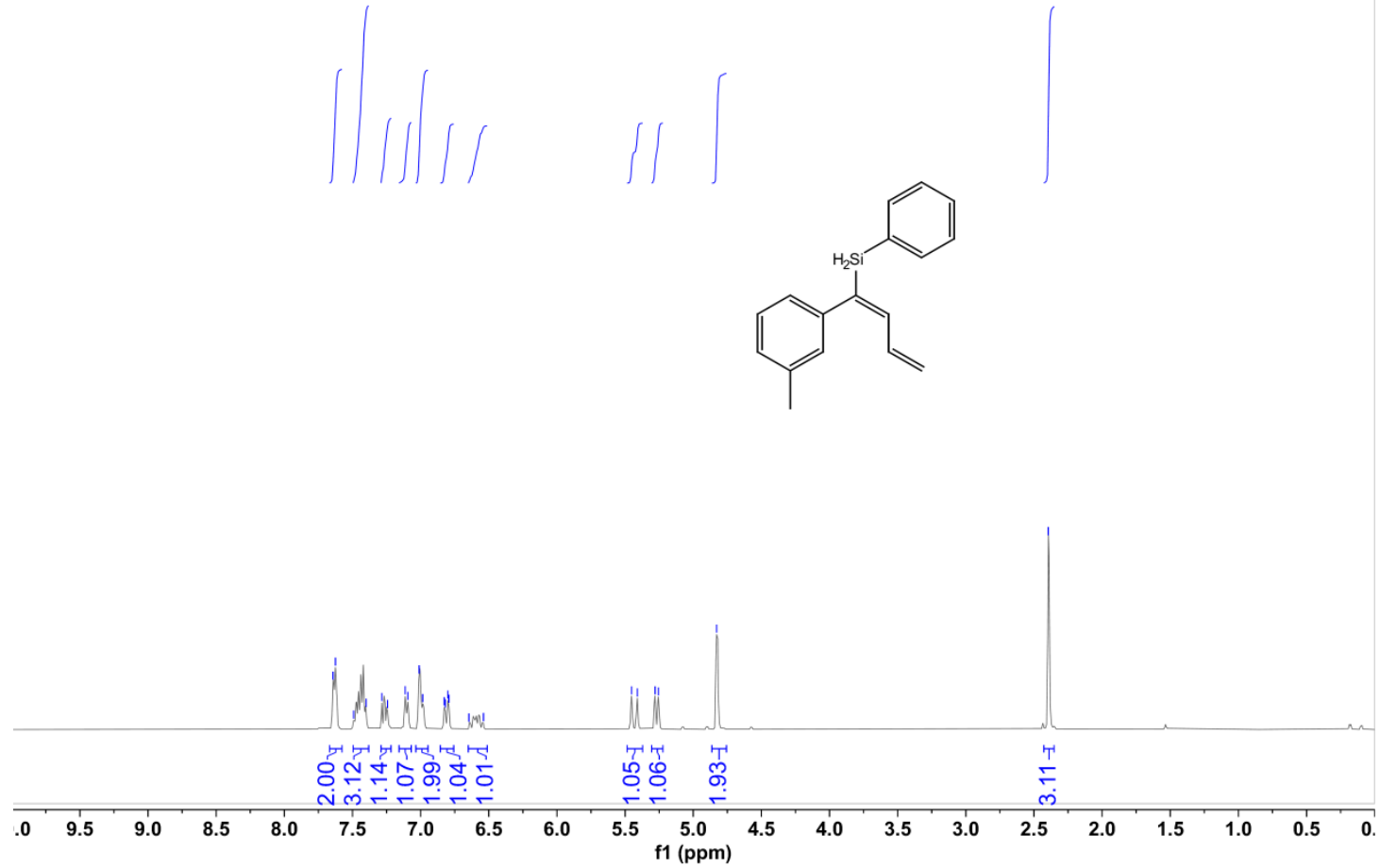

Figure $\mathrm{S} 14{ }^{1} \mathrm{H}$ NMR $\left(\mathrm{CDCl}_{3}, 400 \mathrm{M}\right)$ spectrum of $3 \mathrm{c}$

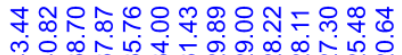

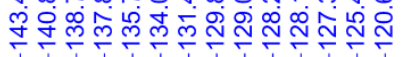

$\stackrel{i n}{i}$

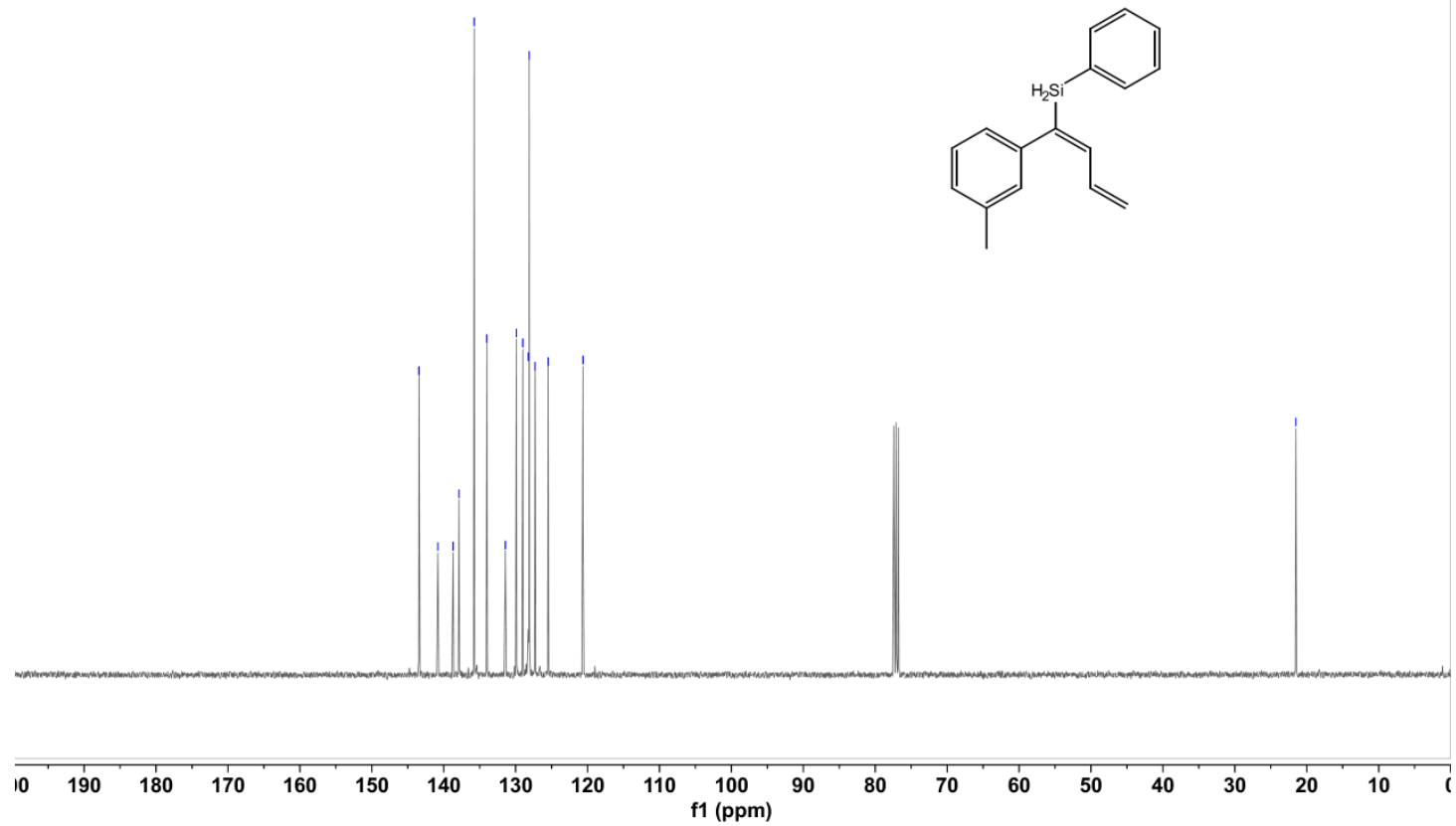

Figure $\mathrm{S15}^{13} \mathrm{C} \mathrm{NMR}\left(\mathrm{CDCl}_{3}, 100 \mathrm{M}\right)$ spectrum of $3 \mathrm{c}$ 


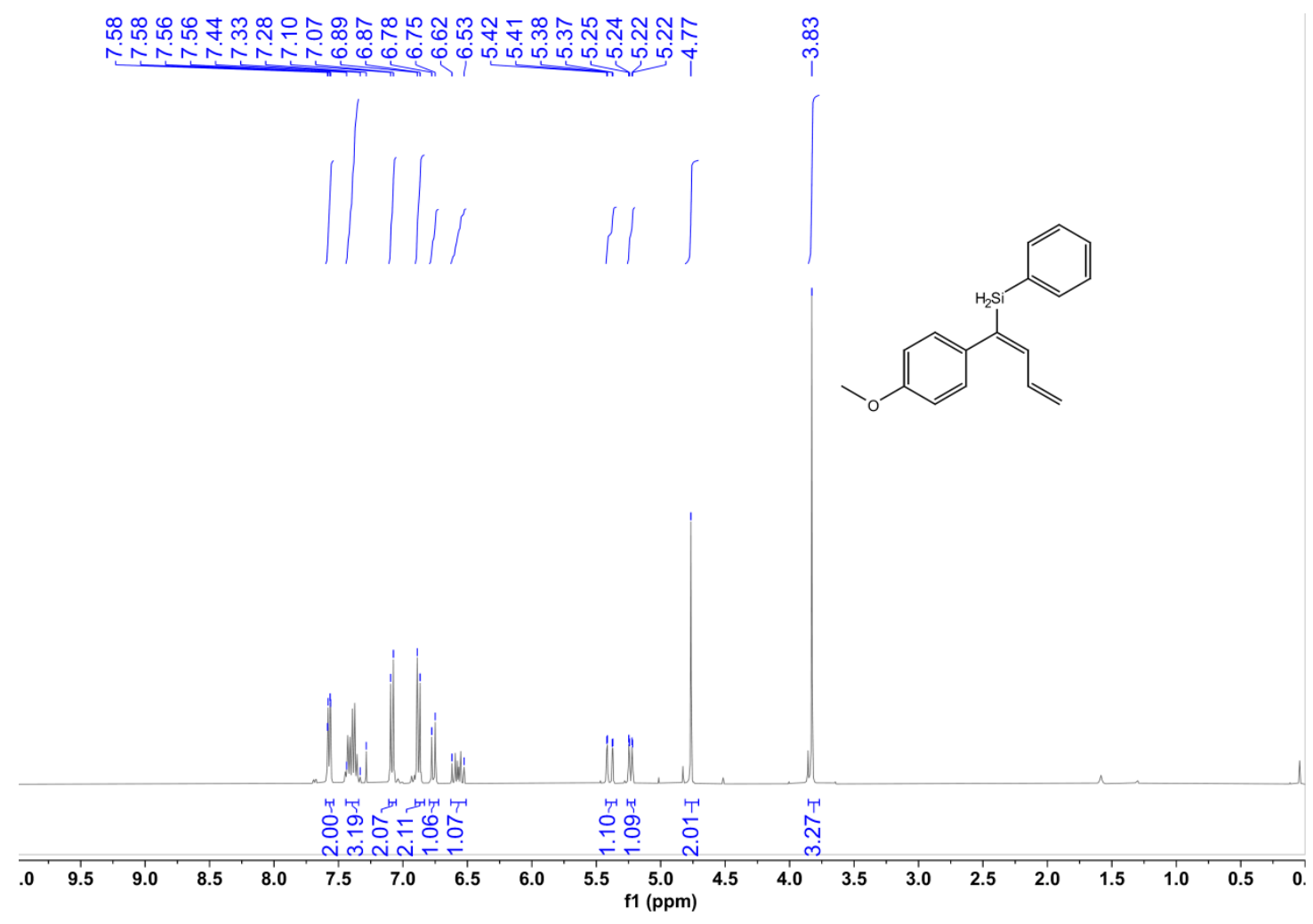

Figure $\mathrm{S16}^{1} \mathrm{H} \mathrm{NMR}\left(\mathrm{CDCl}_{3}, 400 \mathrm{M}\right)$ spectrum of $3 \mathrm{~d}$
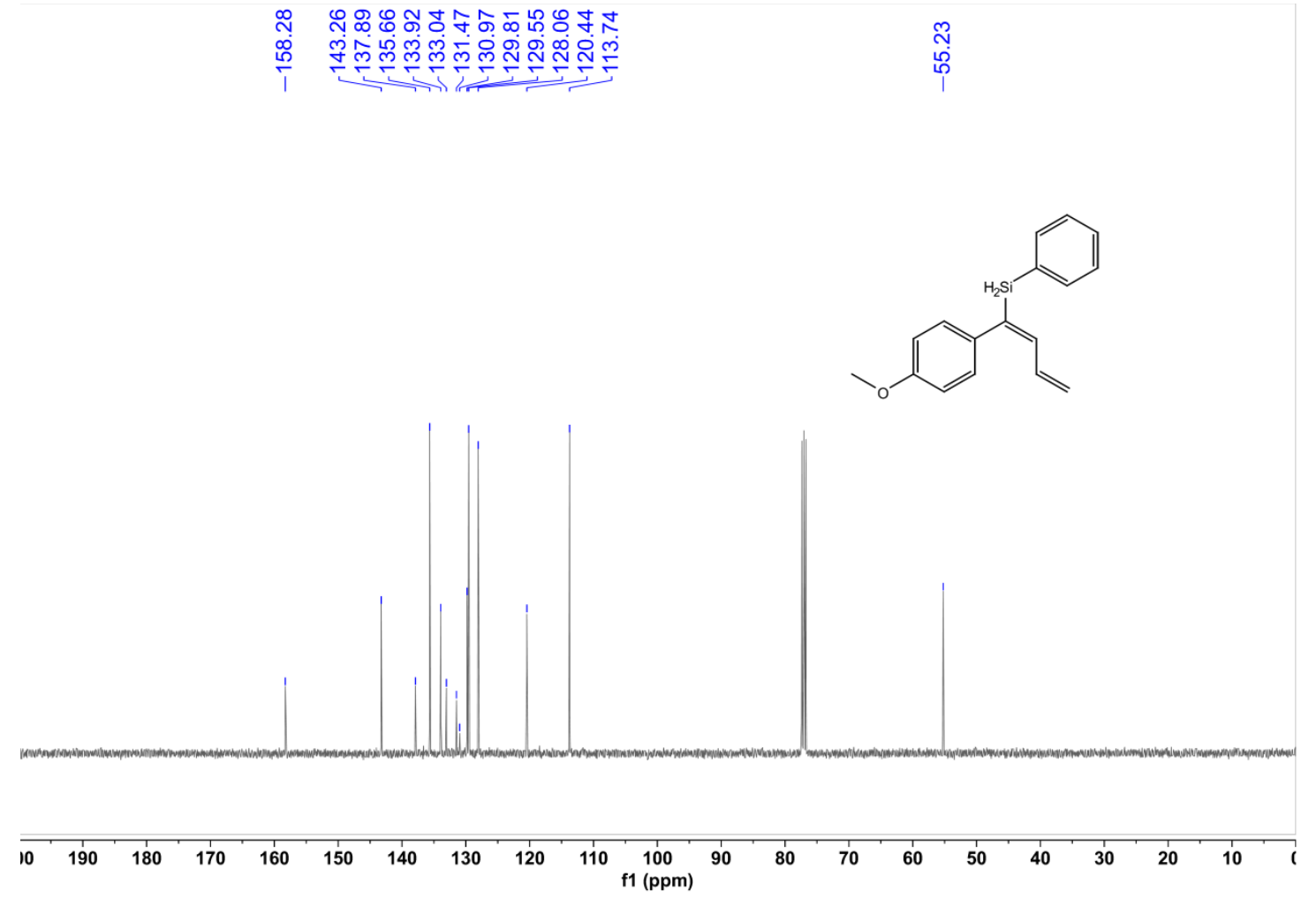

Figure $\mathrm{S17}^{13} \mathrm{C} \mathrm{NMR}\left(\mathrm{CDCl}_{3}, 100 \mathrm{M}\right)$ spectrum of 3d 


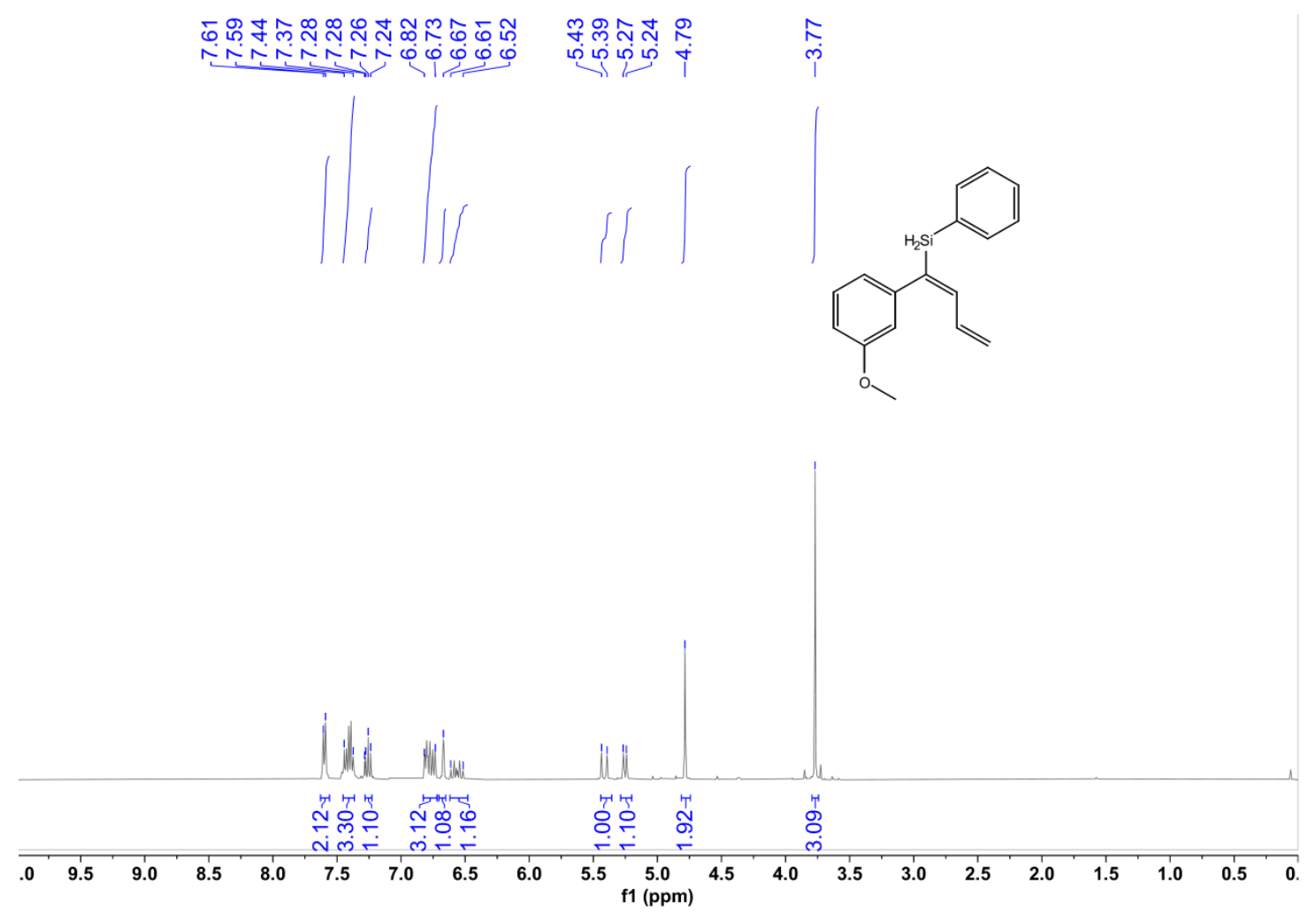

Figure $\mathrm{S18}^{1} \mathrm{H} \mathrm{NMR}\left(\mathrm{CDCl}_{3}, 400 \mathrm{M}\right)$ spectrum of $3 \mathrm{e}$

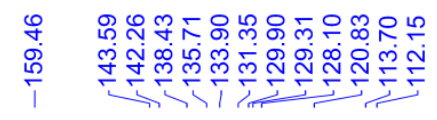

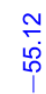

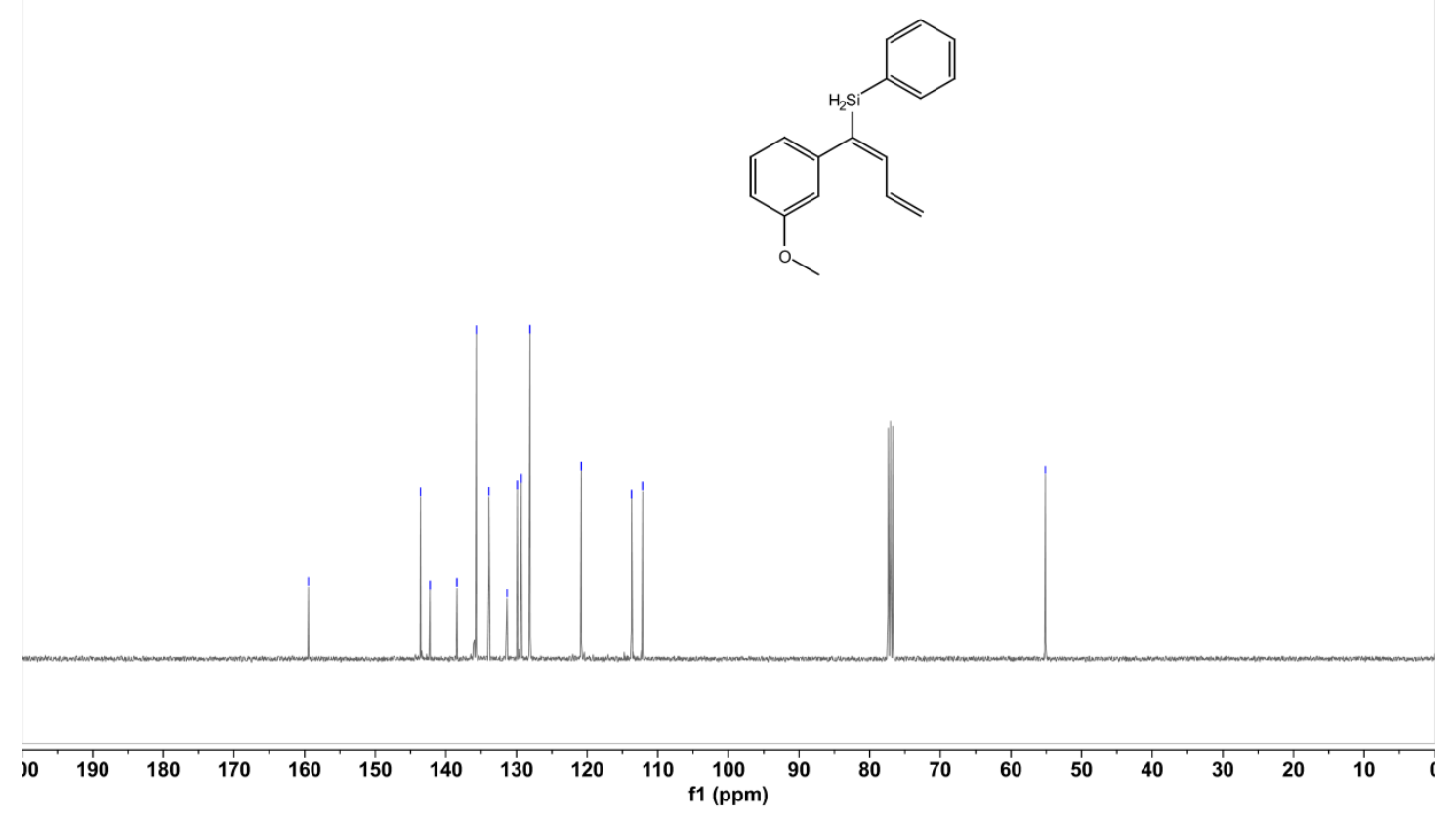

Figure $\mathrm{S19}{ }^{13} \mathrm{C} \mathrm{NMR}\left(\mathrm{CDCl}_{3}, 100 \mathrm{M}\right)$ spectrum of $3 \mathrm{e}$ 


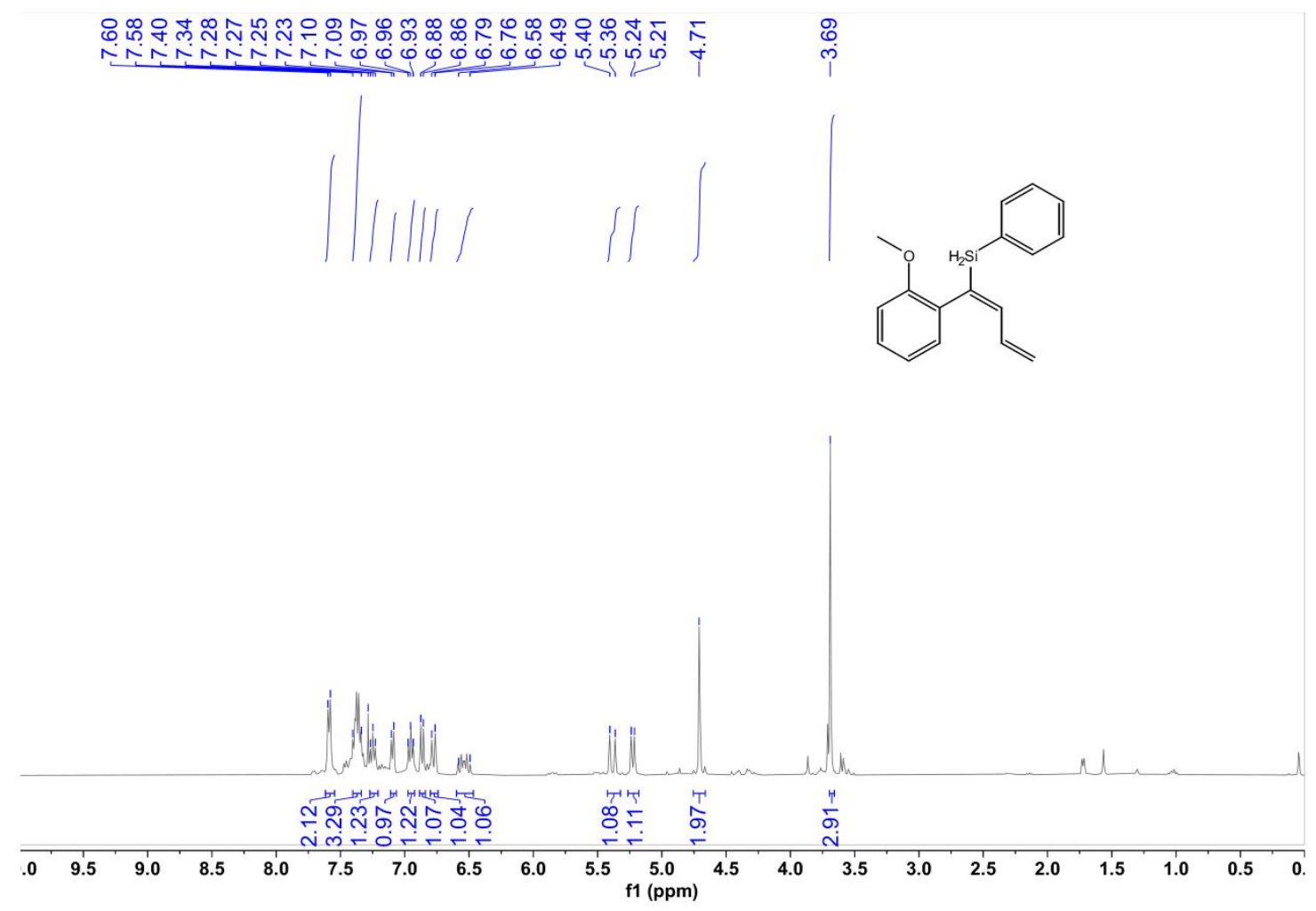

Figure $\mathrm{S20}^{1} \mathrm{H} \mathrm{NMR}\left(\mathrm{CDCl}_{3}, 400 \mathrm{M}\right)$ spectrum of $3 \mathrm{f}$
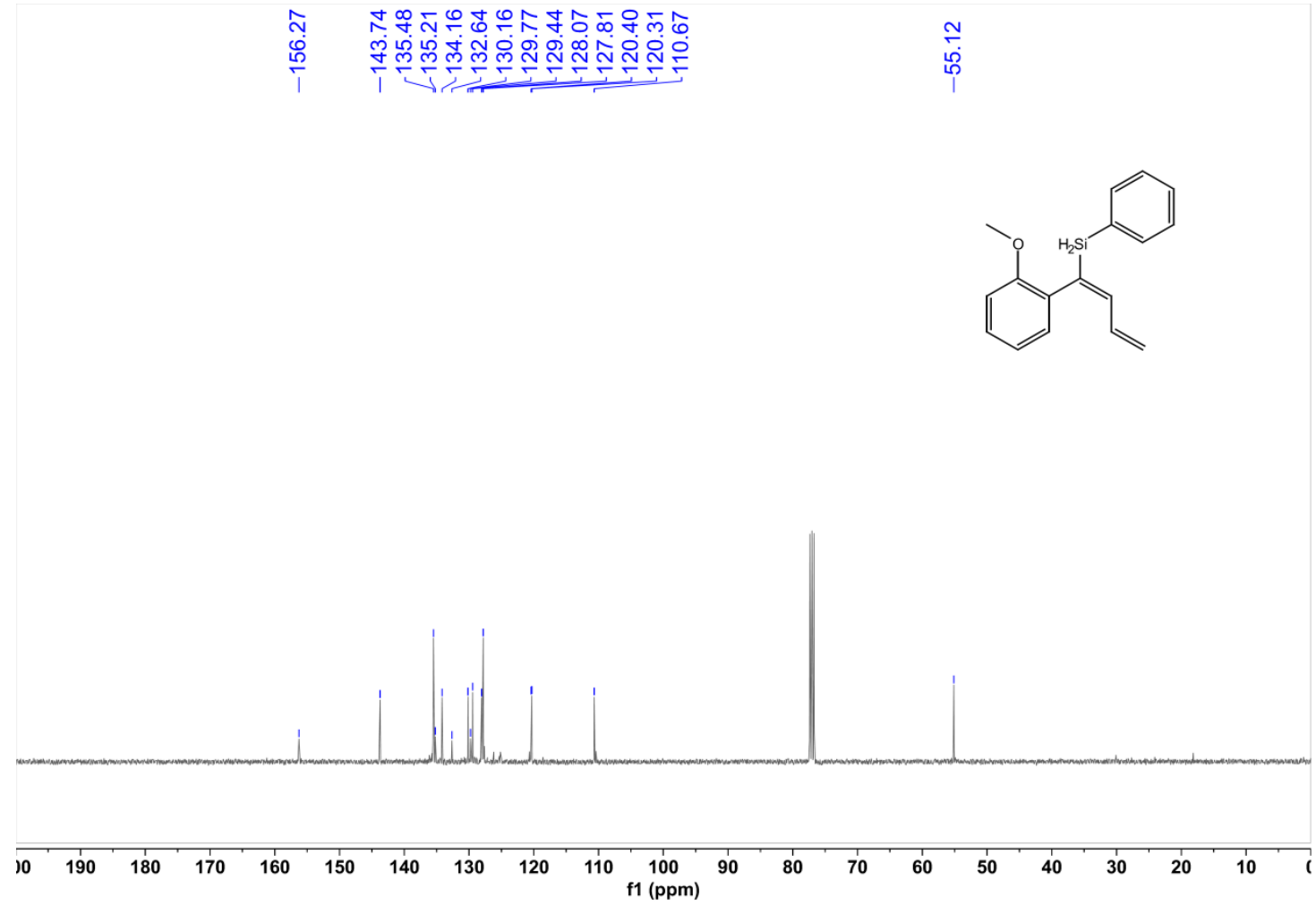

Figure $\mathrm{S} 21{ }^{13} \mathrm{C} \mathrm{NMR}\left(\mathrm{CDCl}_{3}, 100 \mathrm{M}\right)$ spectrum of $3 f$ 


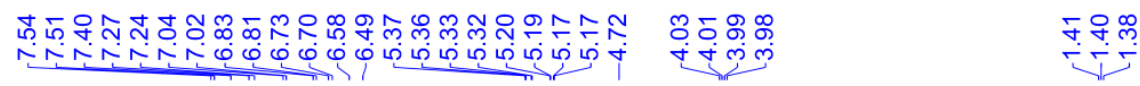
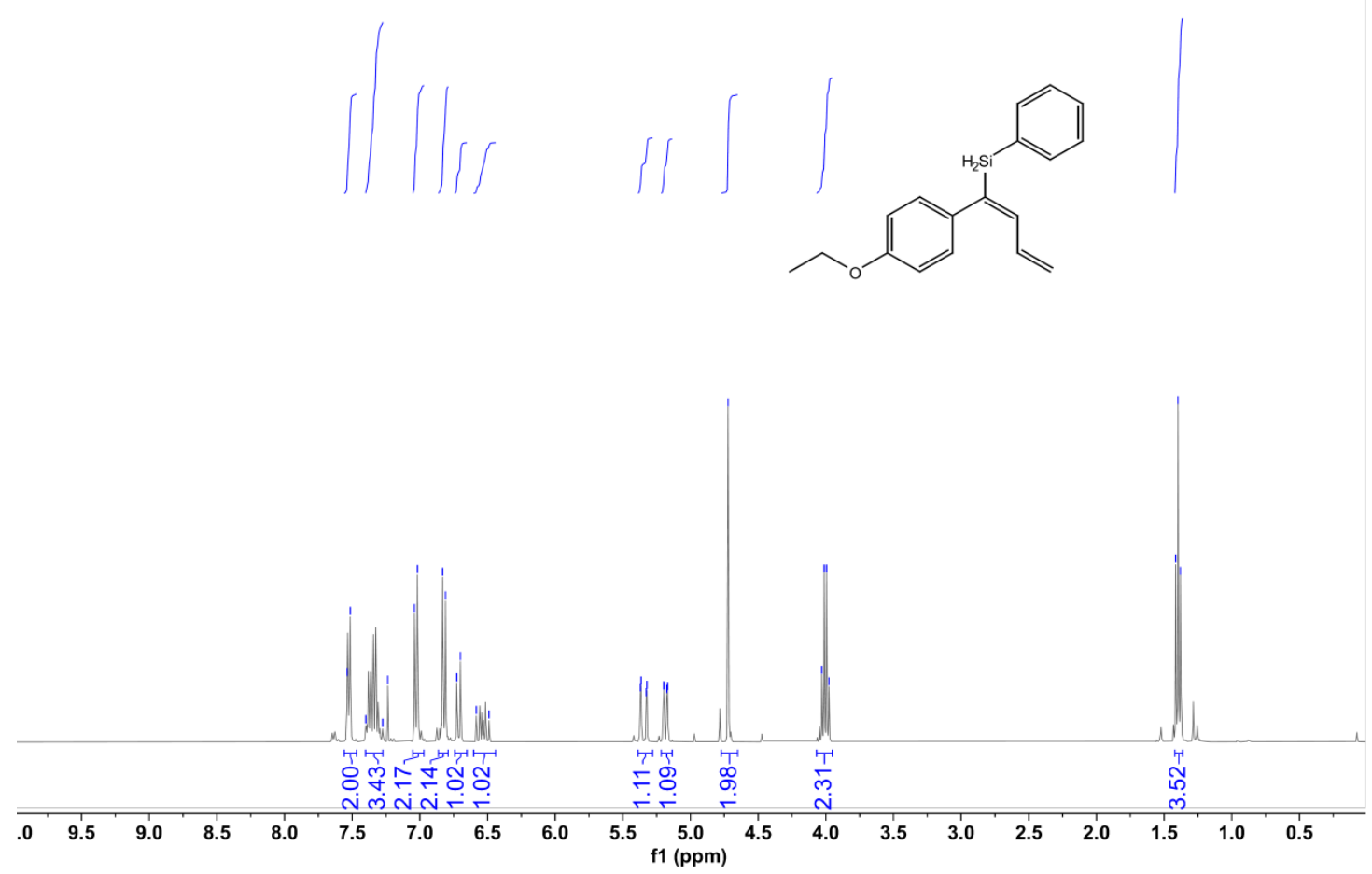

Figure S22 ${ }^{1} \mathrm{H}$ NMR $\left(\mathrm{CDCl}_{3}, 400 \mathrm{M}\right)$ spectrum of $3 \mathrm{~g}$

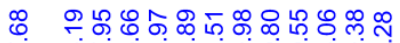

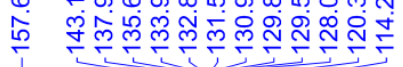
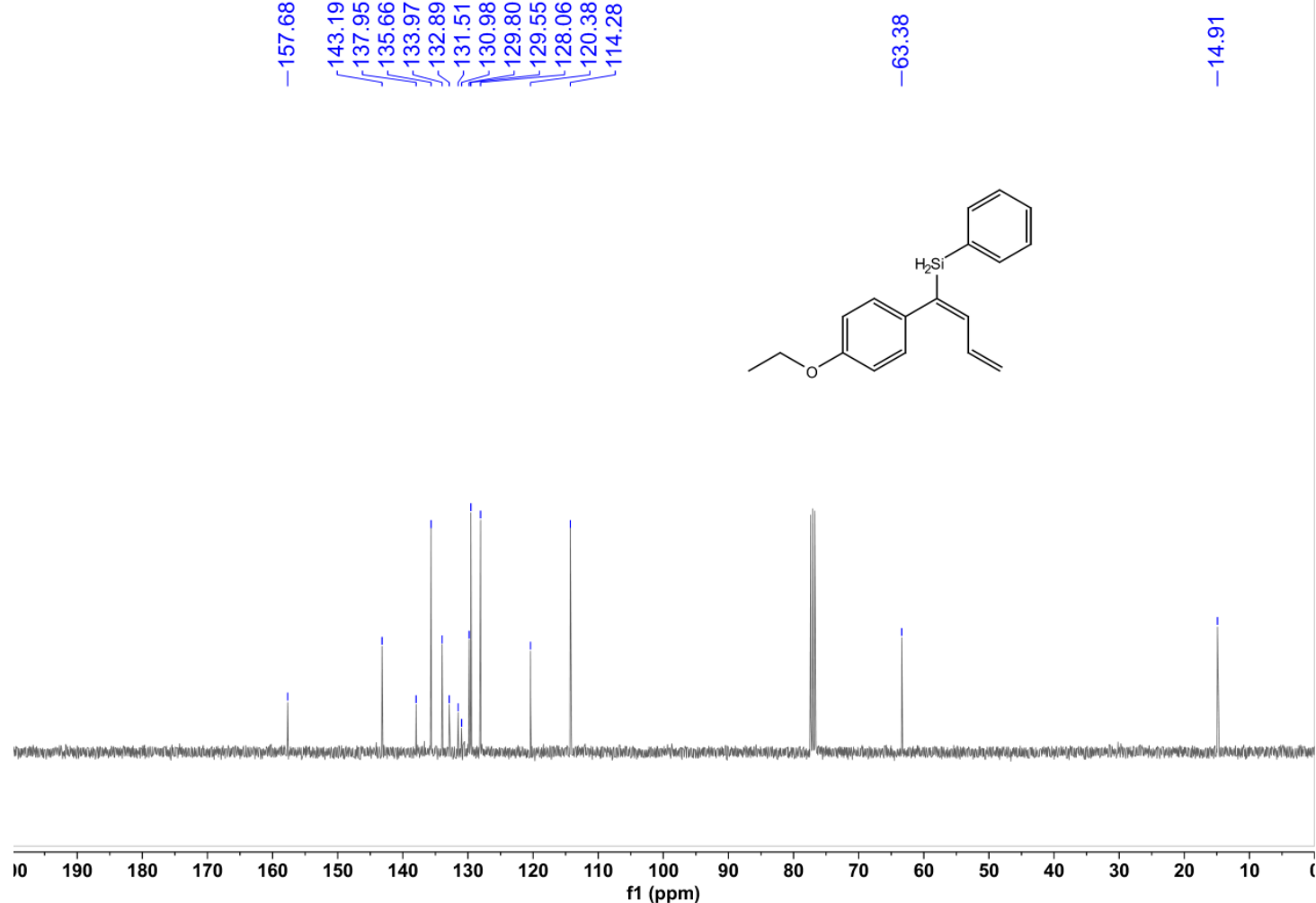

Figure $\mathrm{S23}^{13} \mathrm{C} \mathrm{NMR}\left(\mathrm{CDCl}_{3}, 100 \mathrm{M}\right)$ spectrum of $3 \mathrm{~g}$ 


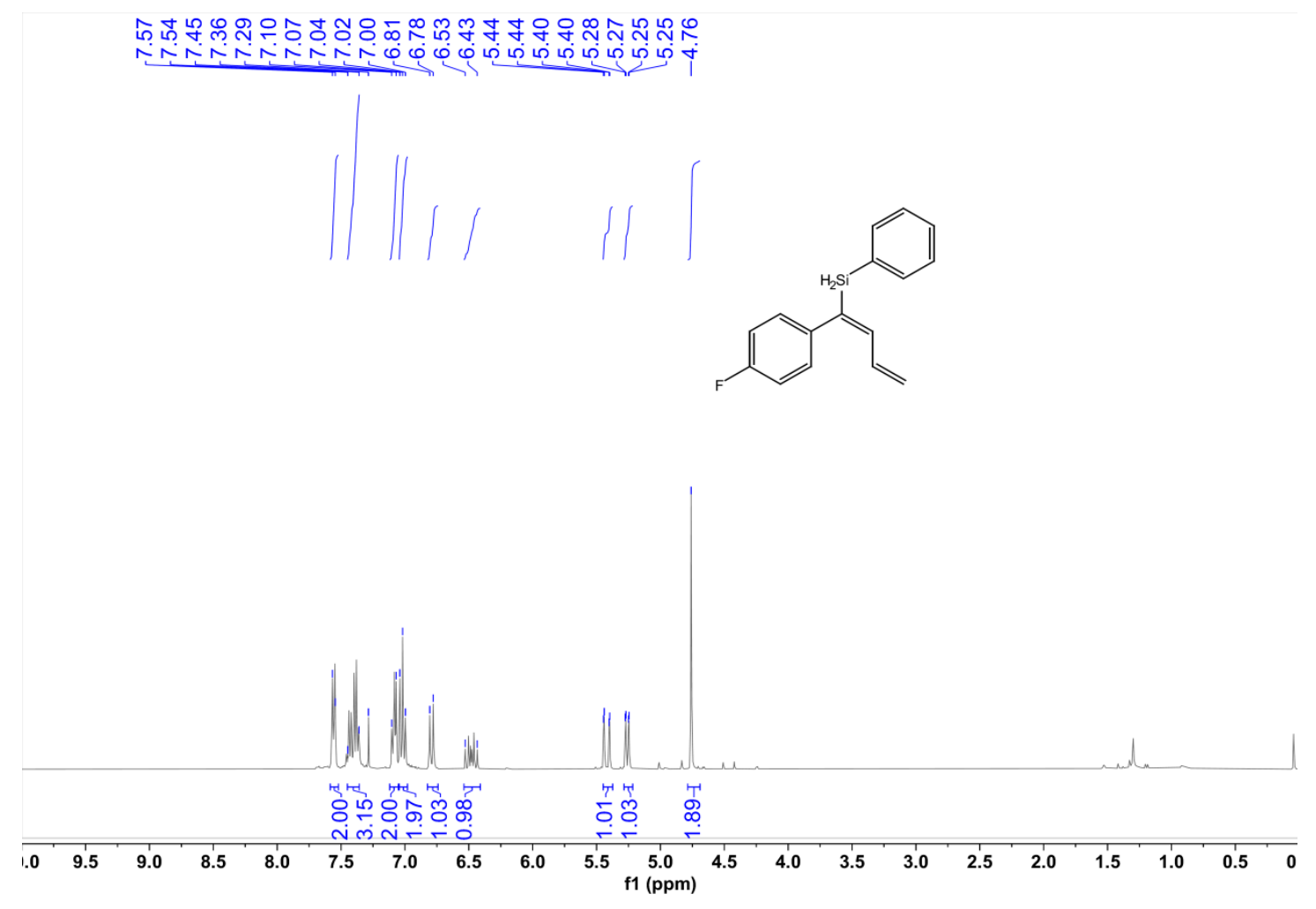

Figure $\mathrm{S} 24{ }^{1} \mathrm{H}$ NMR $\left(\mathrm{CDCl}_{3}, 400 \mathrm{M}\right)$ spectrum of $3 \mathrm{~h}$

œ

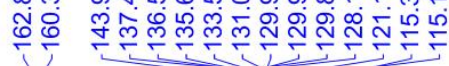
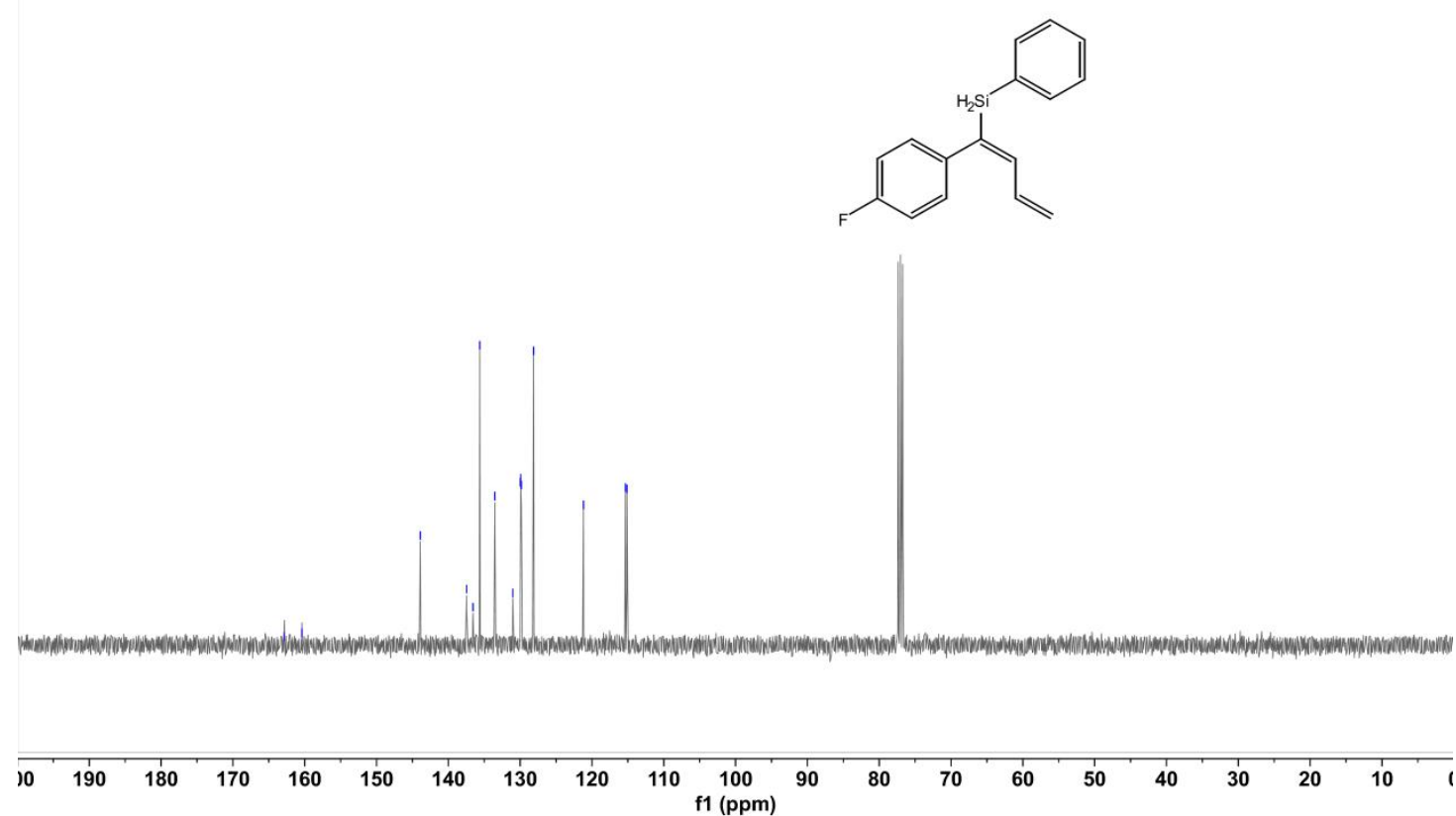

Figure $\mathrm{S25}{ }^{13} \mathrm{C} \mathrm{NMR}\left(\mathrm{CDCl}_{3}, 100 \mathrm{M}\right)$ spectrum of $3 \mathrm{~h}$ 


\section{$\frac{\text { D }}{\frac{0}{5}}$}<smiles>C=C/C=C(/Nc1ccccc1)c1ccc(F)cc1</smiles>

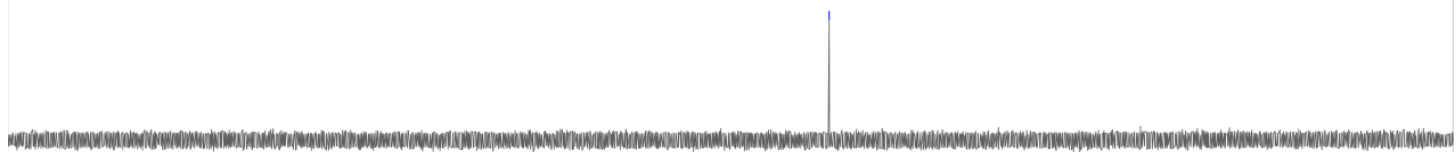

\begin{tabular}{rlllllllllllllllllllllllllllll}
\hline 10 & 0 & -10 & -20 & -30 & -40 & -50 & -60 & -70 & -80 & -90 & -100 & -110 & -120 & -130 & -140 & -150 & -160 & -170 & -180 & -190 & -200 & -210 \\
\hline
\end{tabular}

Figure S26 ${ }^{19}$ F NMR $\left(\mathrm{CDCl}_{3}, 376 \mathrm{MHz}\right)$ spectrum of $3 \mathrm{~h}$

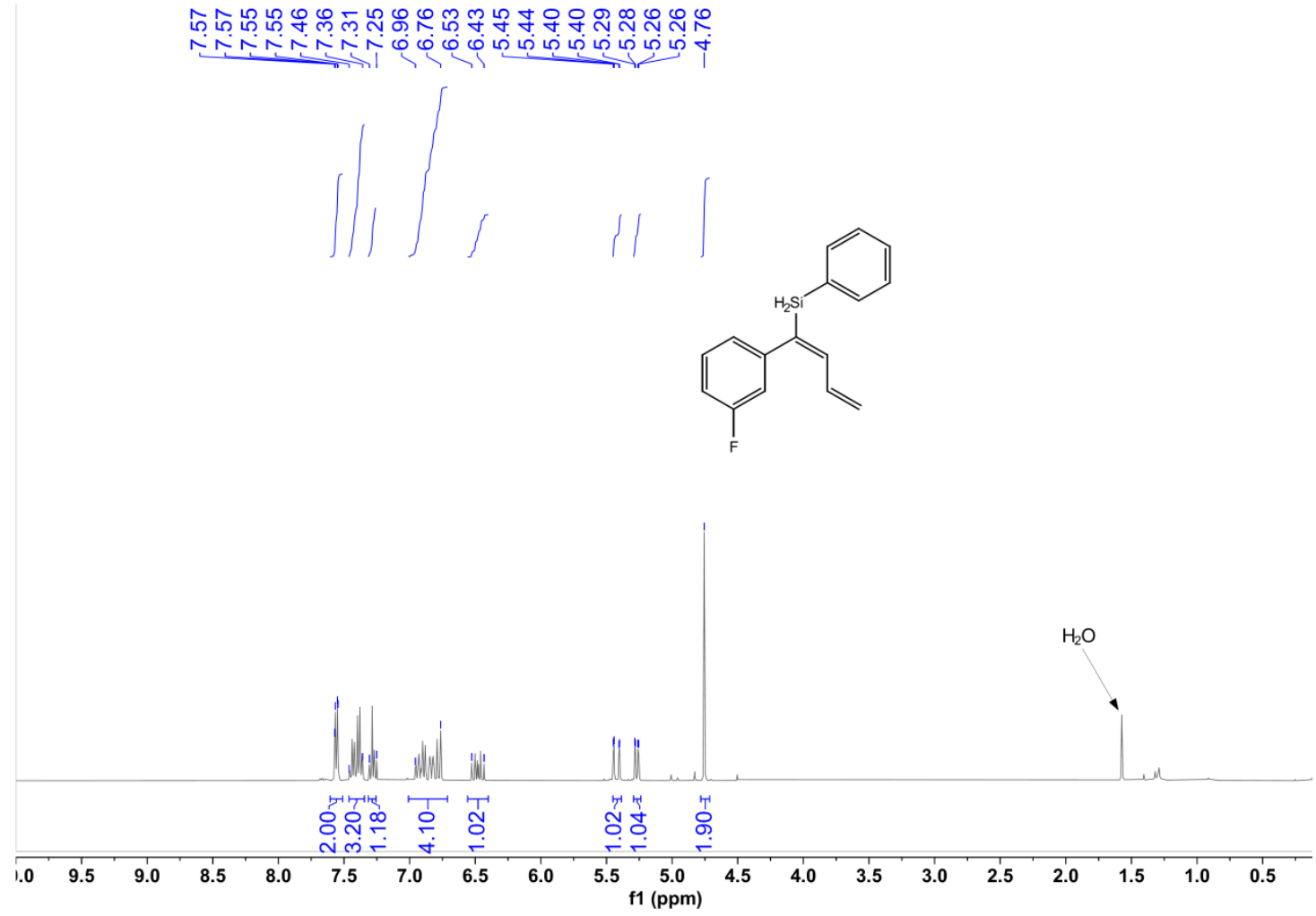

Figure $\mathrm{S} 27{ }^{1} \mathrm{H}$ NMR $\left(\mathrm{CDCl}_{3}, 400 \mathrm{M}\right)$ spectrum of $3 \mathrm{i}$ 


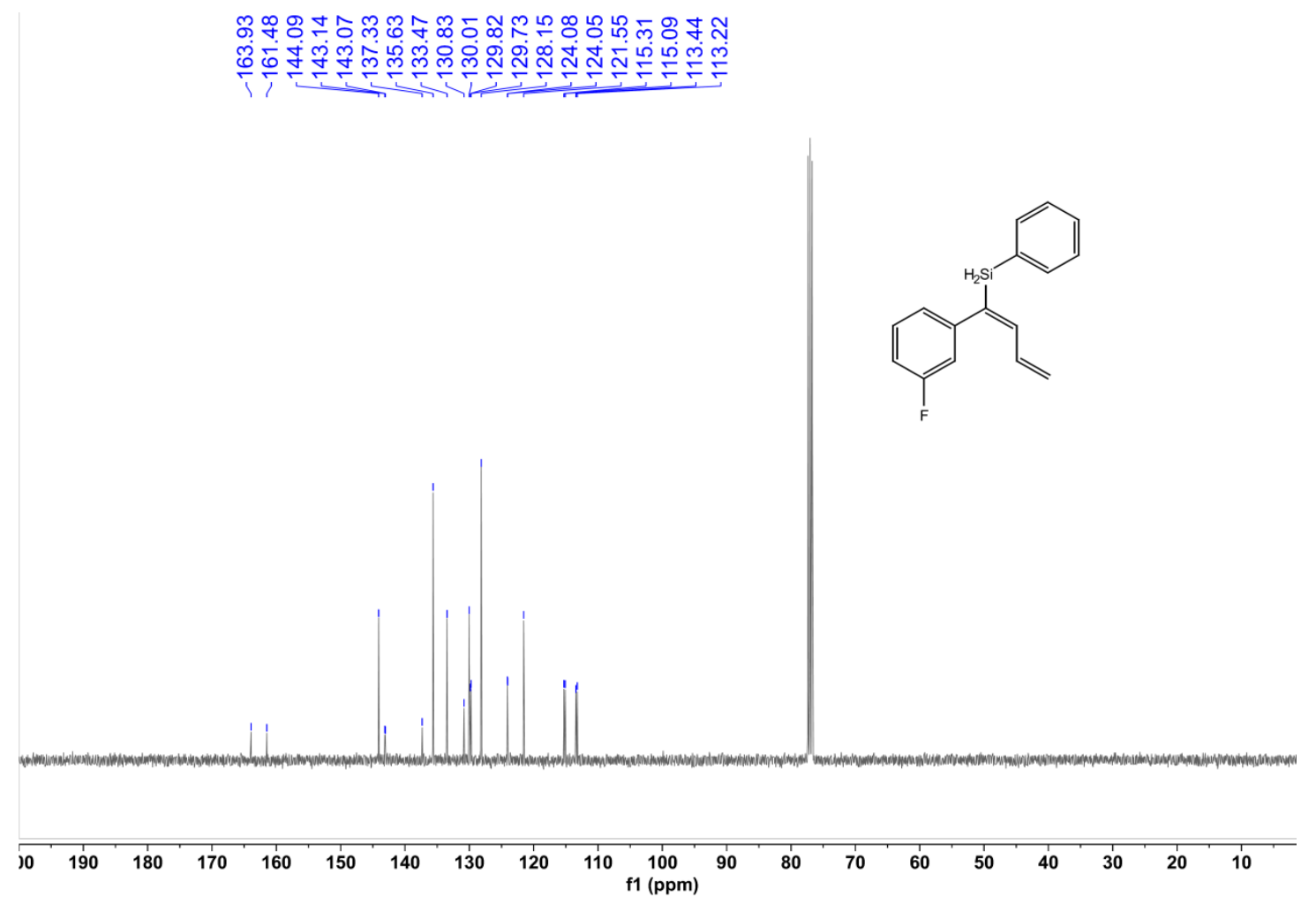

Figure $\mathrm{S} 28{ }^{13} \mathrm{C}$ NMR $\left(\mathrm{CDCl}_{3}, 100 \mathrm{M}\right)$ spectrum of $3 \mathrm{i}$

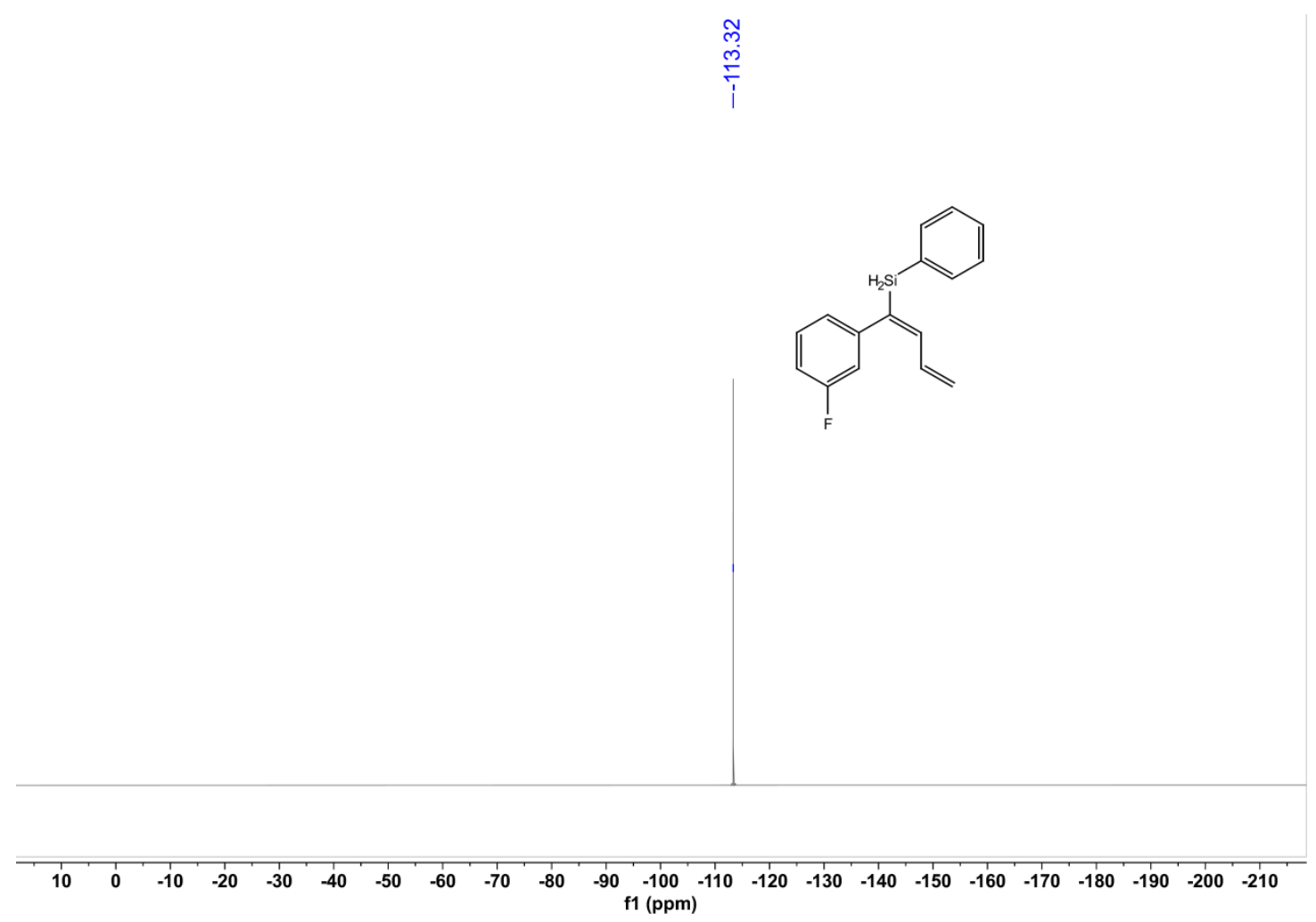

Figure $\mathrm{S29}^{19} \mathrm{~F}$ NMR $\left(\mathrm{CDCl}_{3}, 376 \mathrm{MHz}\right)$ spectrum of 3i 


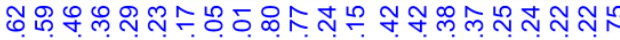

个.r.
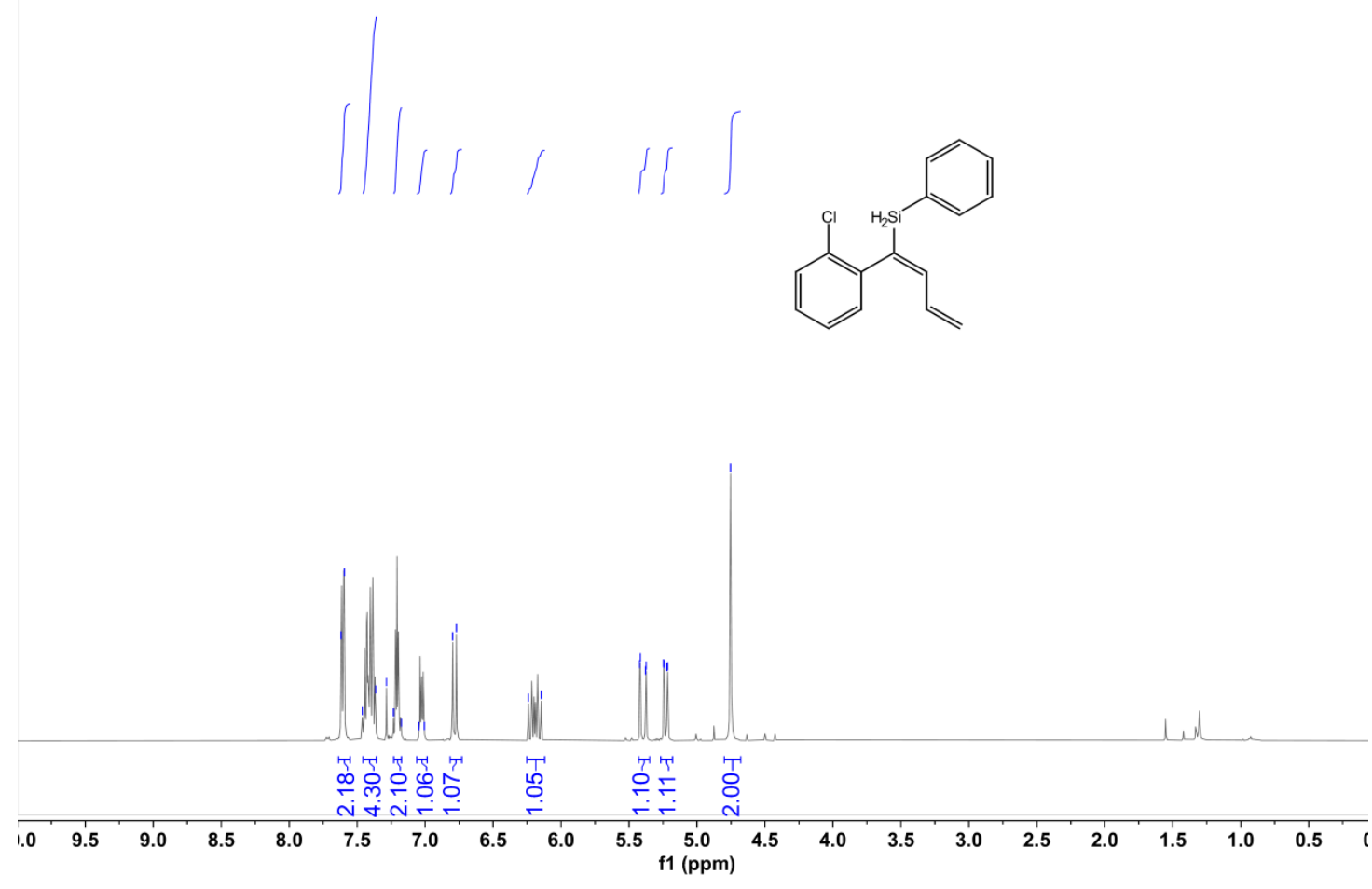

Figure $\mathrm{S30}{ }^{1} \mathrm{H}$ NMR $\left(\mathrm{CDCl}_{3}, 400 \mathrm{M}\right)$ spectrum of $3 \mathrm{j}$
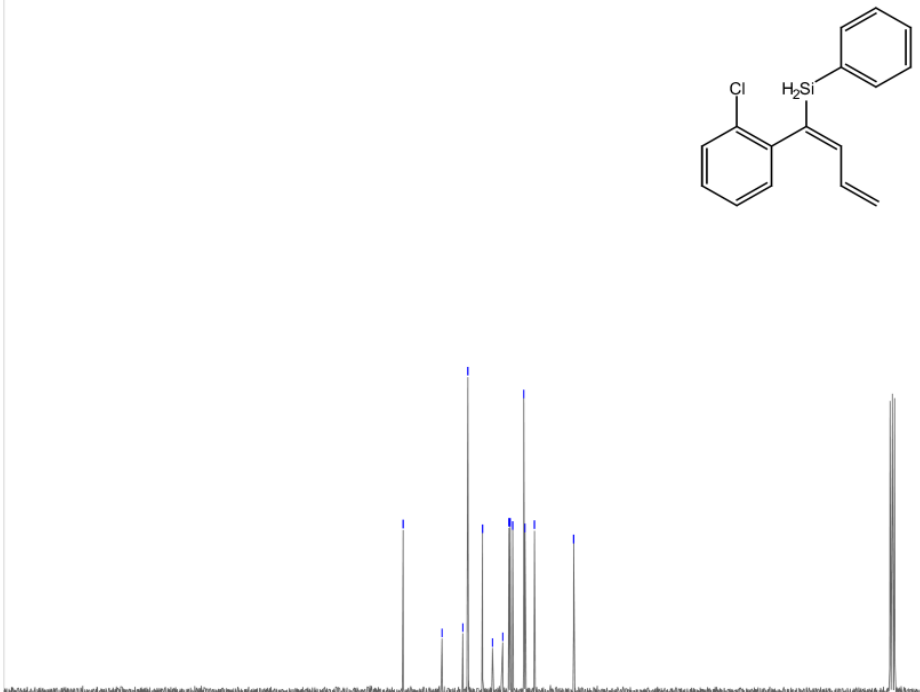

30
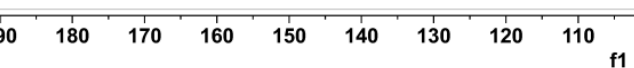

100
$\mathrm{f} 1(\mathrm{ppm})$

Figure $\mathrm{S31}{ }^{13} \mathrm{C} \mathrm{NMR}\left(\mathrm{CDCl}_{3}, 100 \mathrm{M}\right)$ spectrum of $3 \mathrm{j}$ 


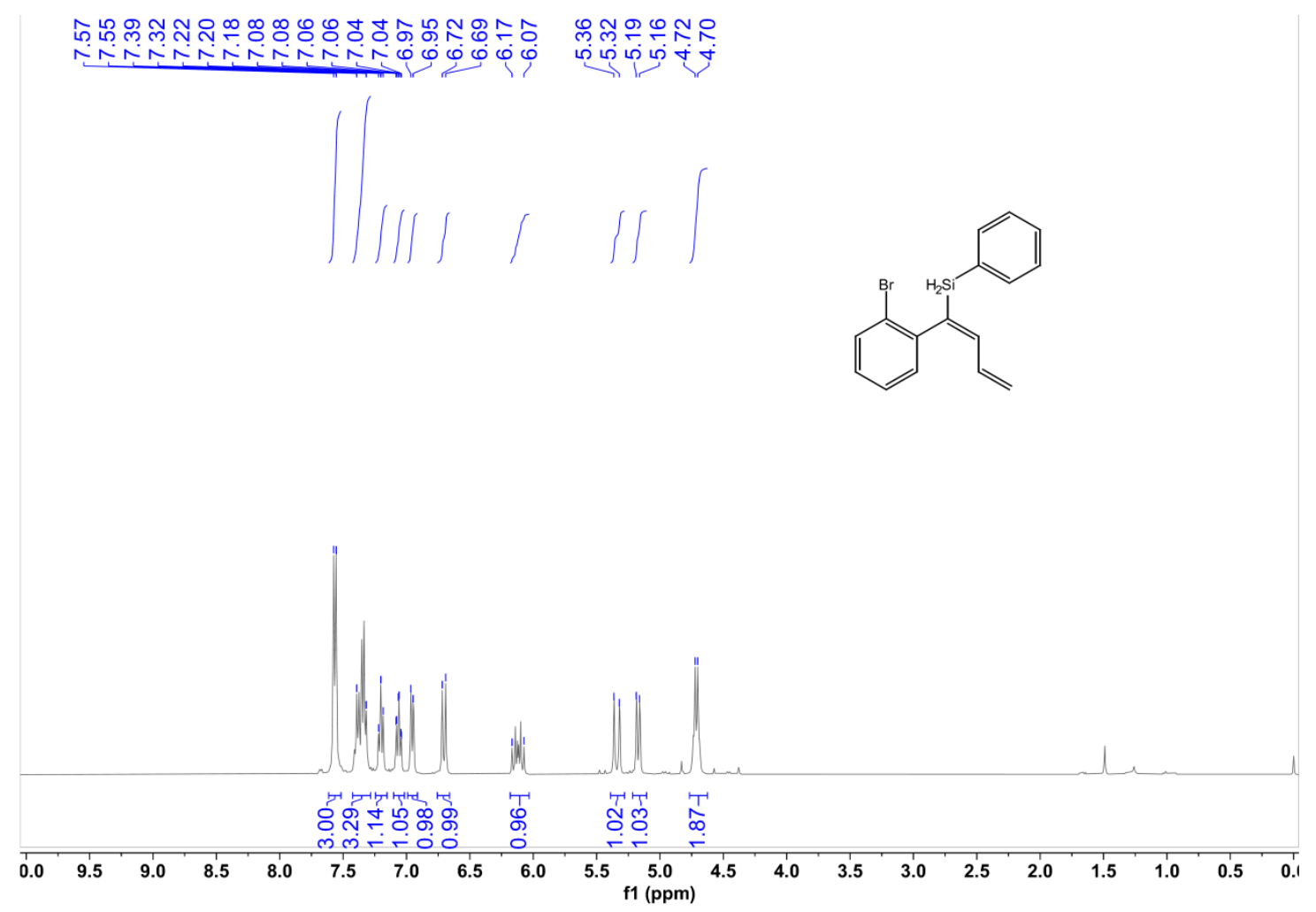

Figure $\mathrm{S32}{ }^{1} \mathrm{H} \mathrm{NMR}\left(\mathrm{CDCl}_{3}, 400 \mathrm{M}\right)$ spectrum of $3 \mathrm{k}$

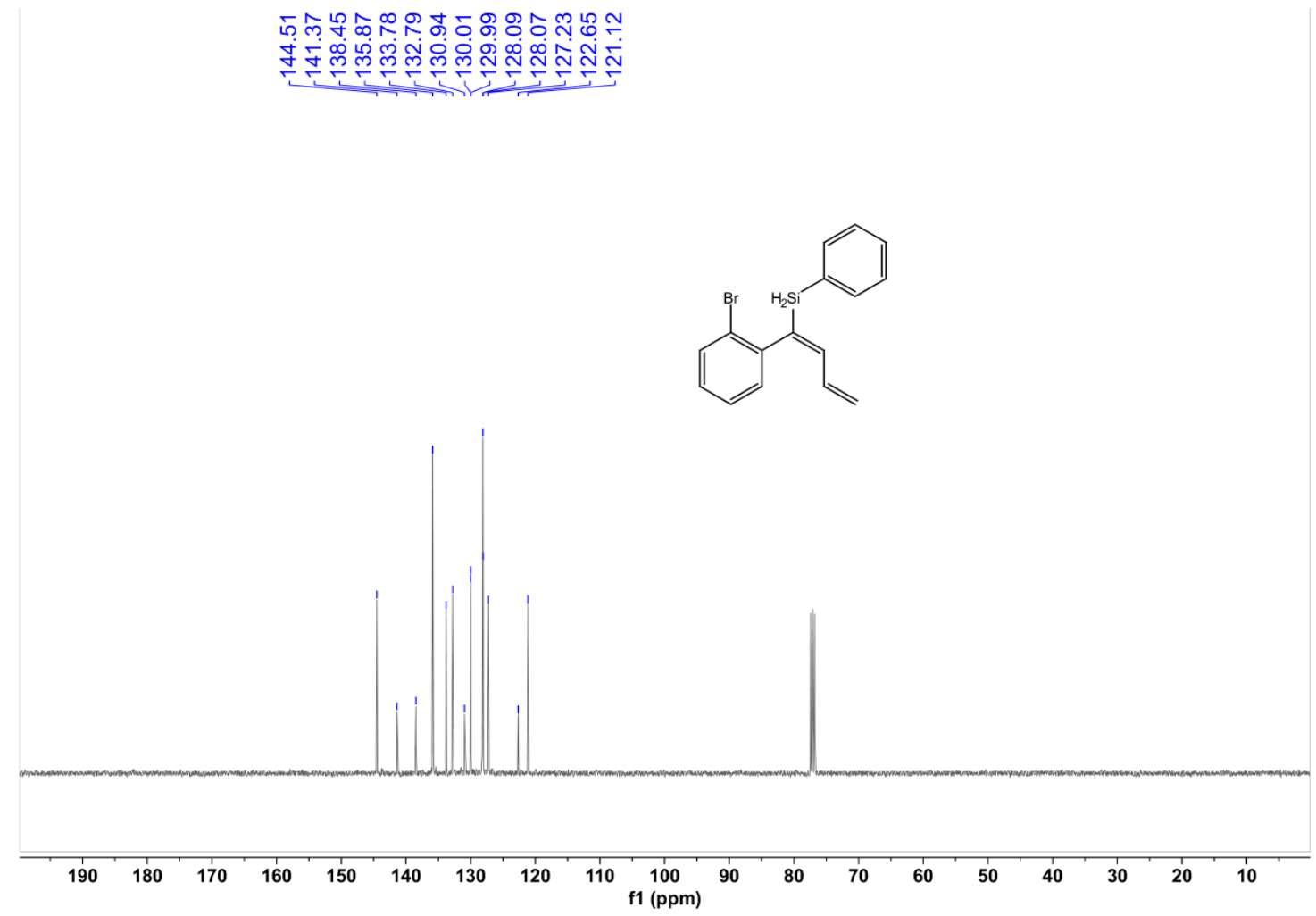

Figure $\mathrm{S33}^{13} \mathrm{C} \mathrm{NMR}\left(\mathrm{CDCl}_{3}, 100 \mathrm{M}\right)$ spectrum of $3 \mathrm{k}$ 


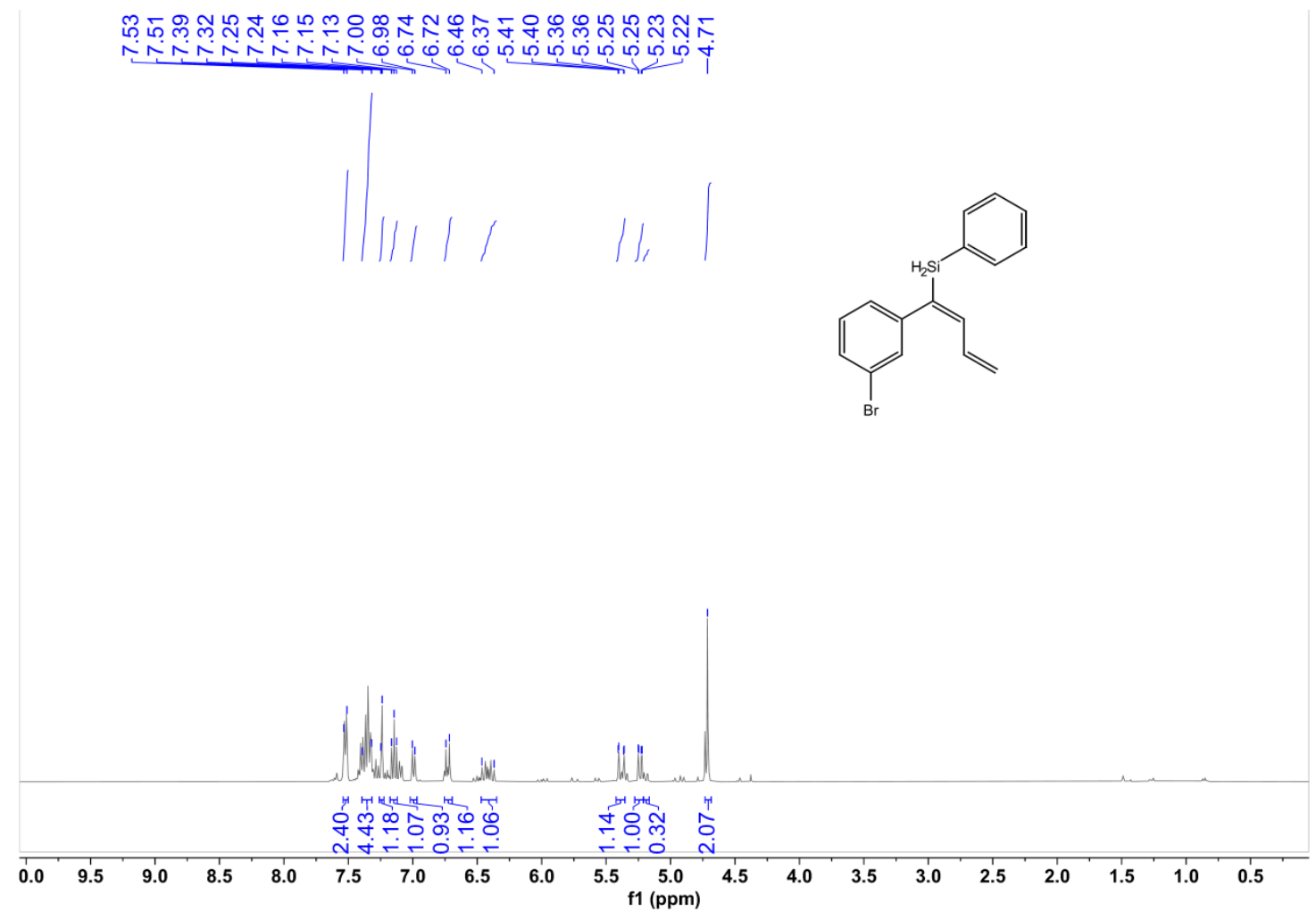

Figure S34 ${ }^{1} \mathrm{H}$ NMR $\left(\mathrm{CDCl}_{3}, 400 \mathrm{M}\right)$ spectrum of 31

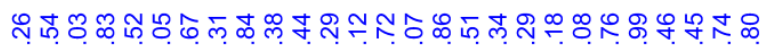

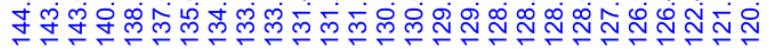
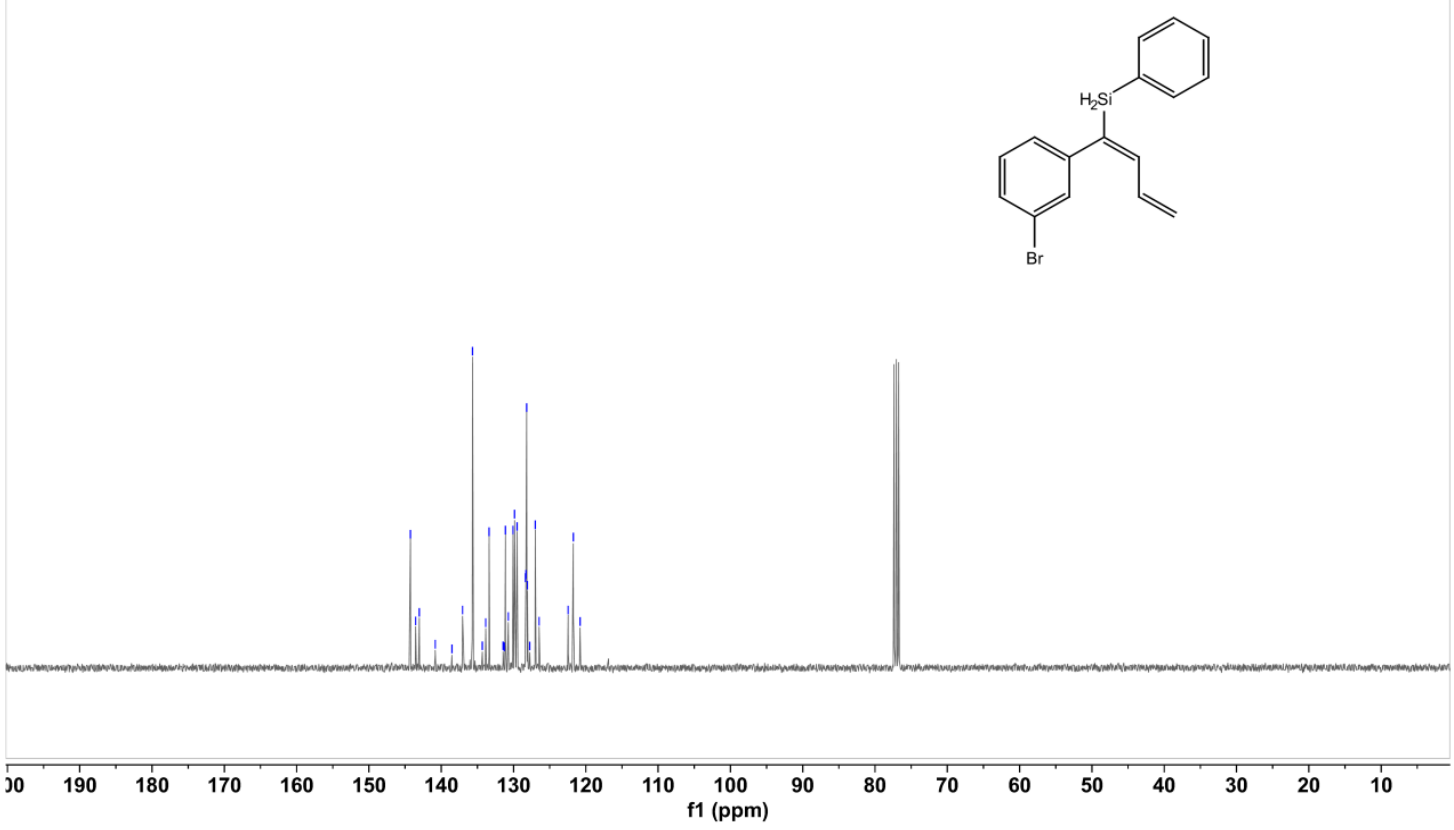

Figure $\mathrm{S35}^{13} \mathrm{C} \mathrm{NMR}\left(\mathrm{CDCl}_{3}, 100 \mathrm{M}\right)$ spectrum of 31 


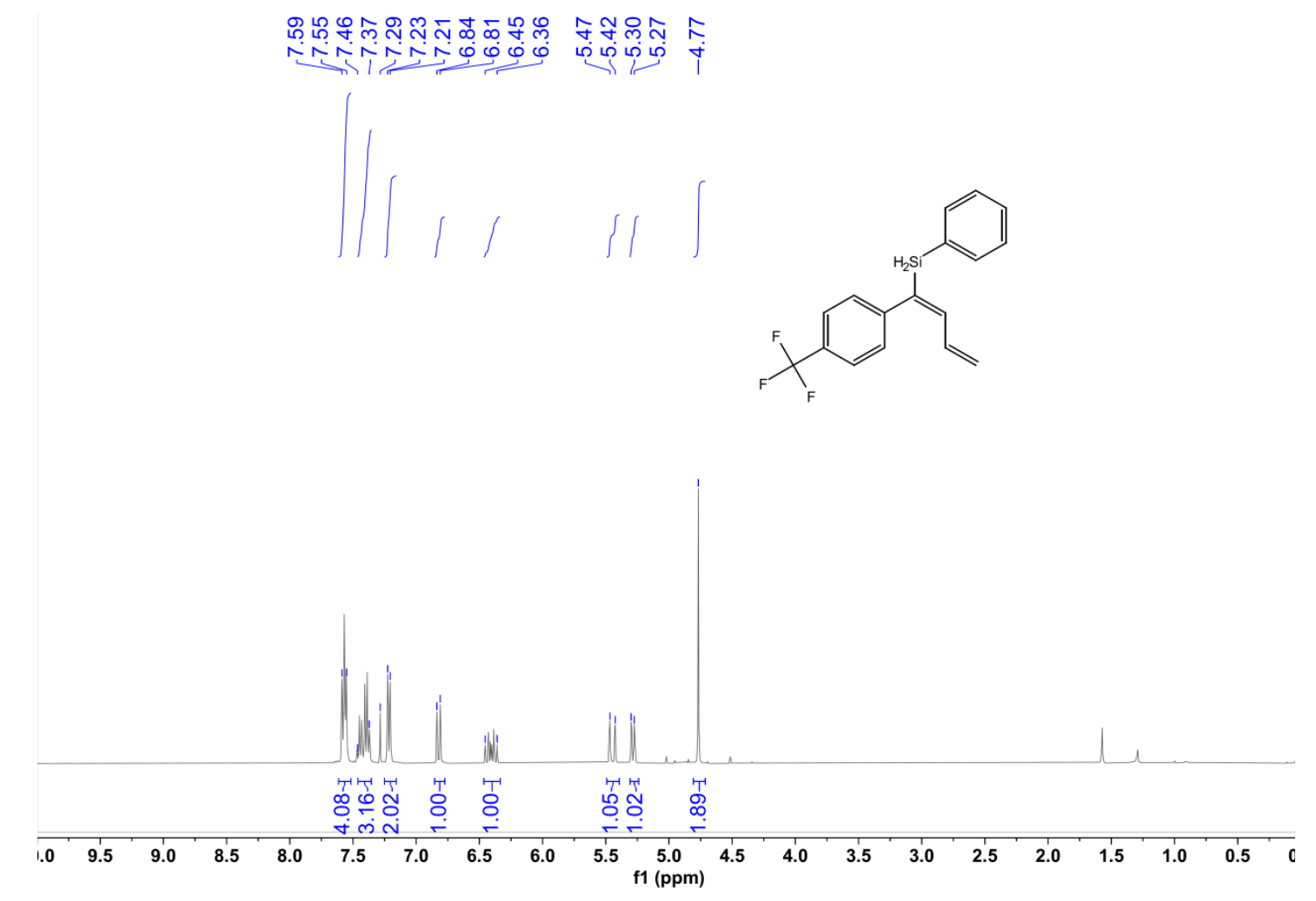

Figure S36 ${ }^{1} \mathrm{H}$ NMR $\left(\mathrm{CDCl}_{3}, 400 \mathrm{M}\right)$ spectrum of $3 \mathrm{~m}$

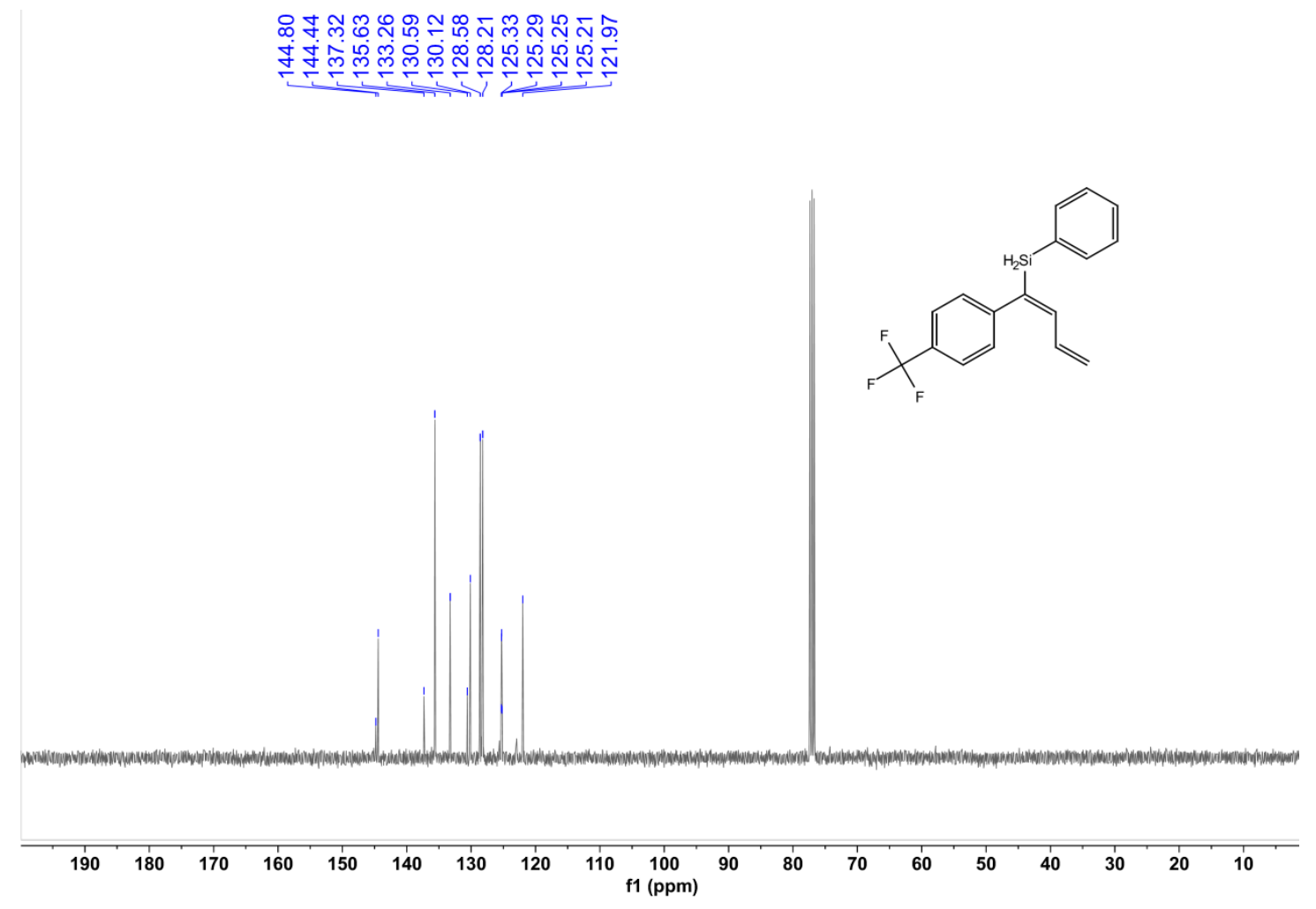

Figure $\mathrm{S37}{ }^{13} \mathrm{C} \mathrm{NMR}\left(\mathrm{CDCl}_{3}, 100 \mathrm{M}\right)$ spectrum of $3 \mathrm{~m}$ 


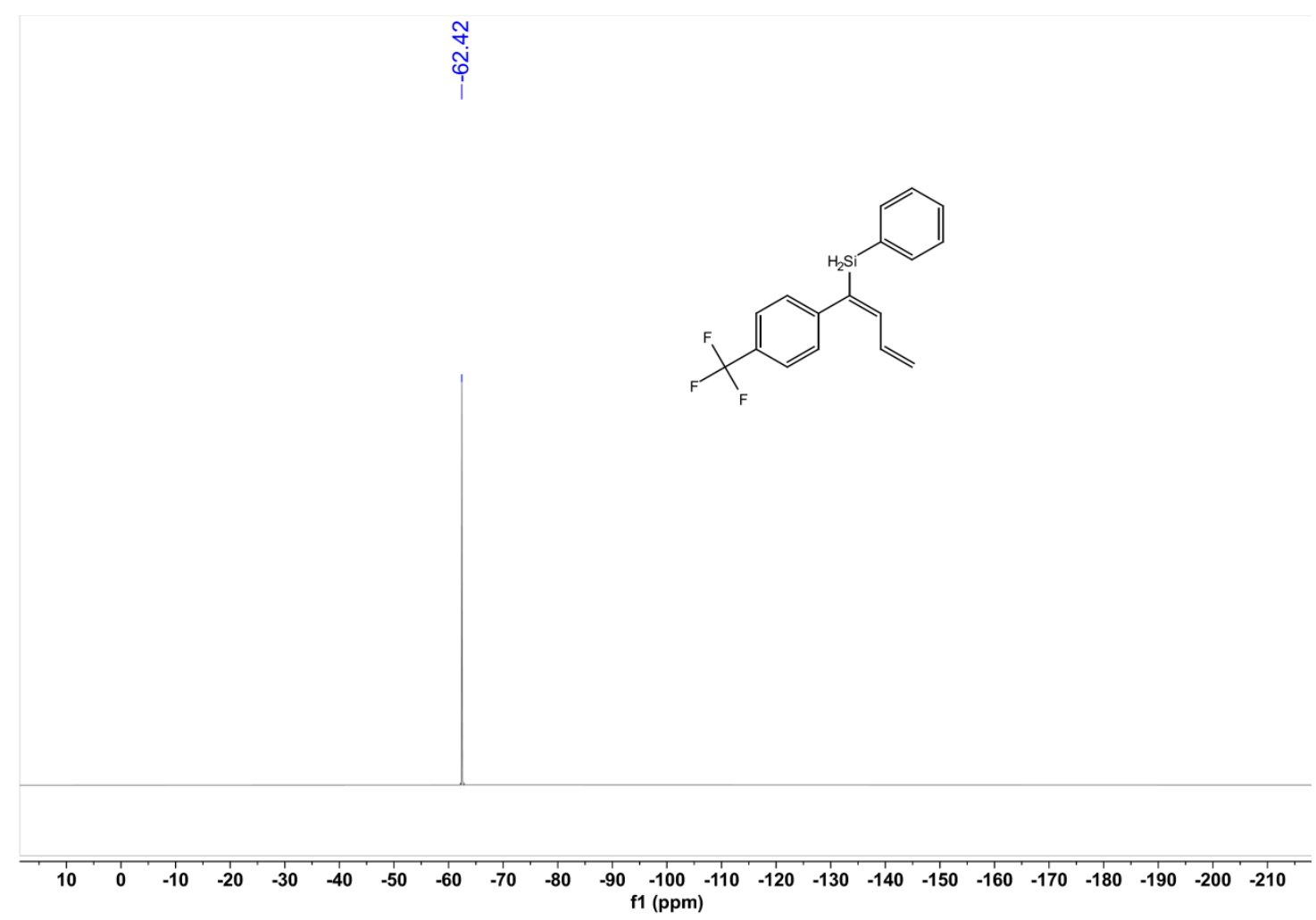

Figure $\mathrm{S38}^{19} \mathrm{~F}$ NMR $\left(\mathrm{CDCl}_{3}, 376 \mathrm{MHz}\right)$ spectrum of $3 \mathrm{~m}$
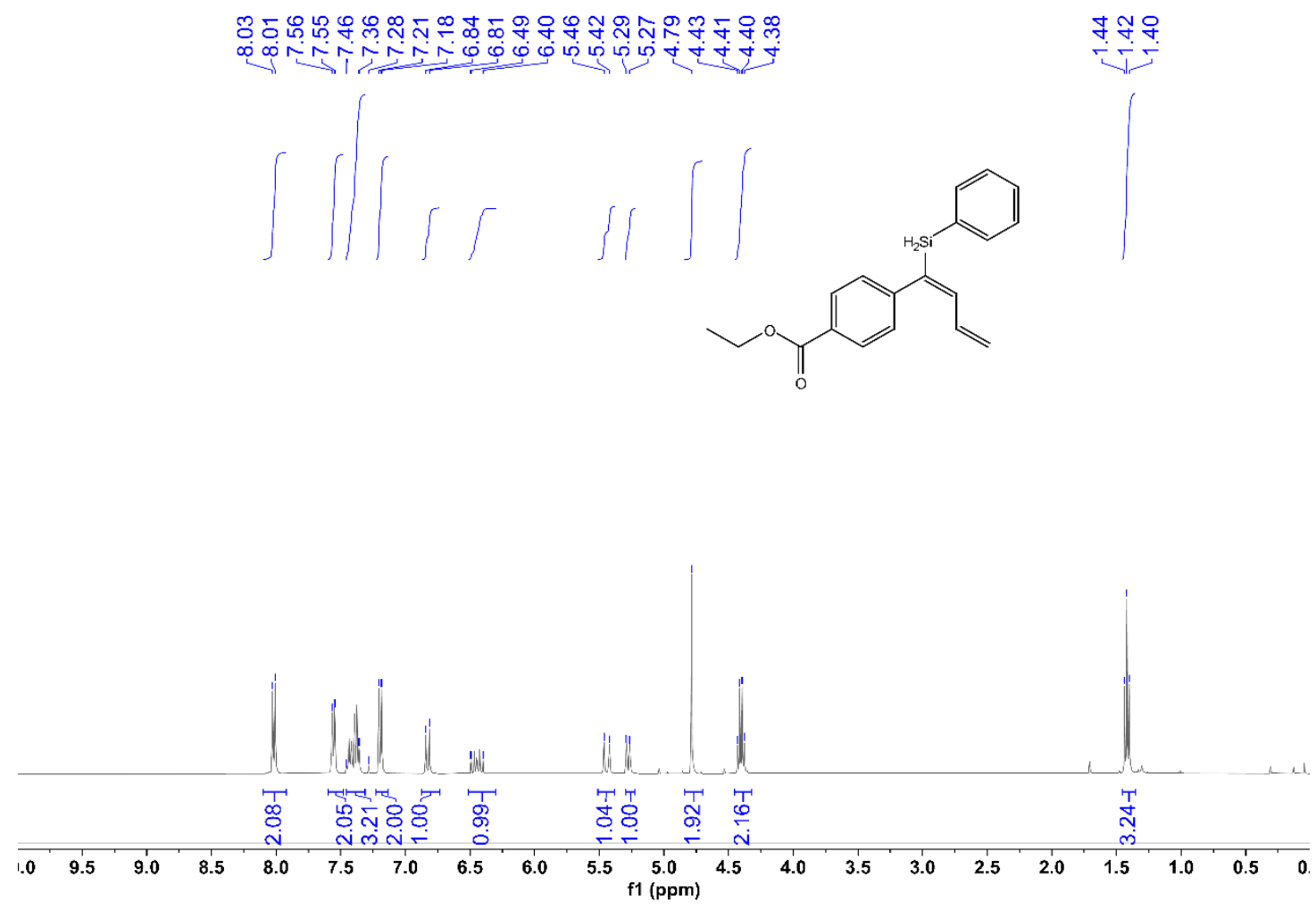

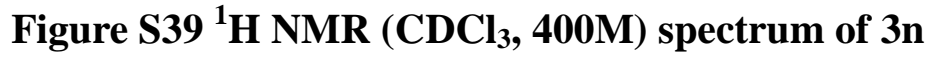




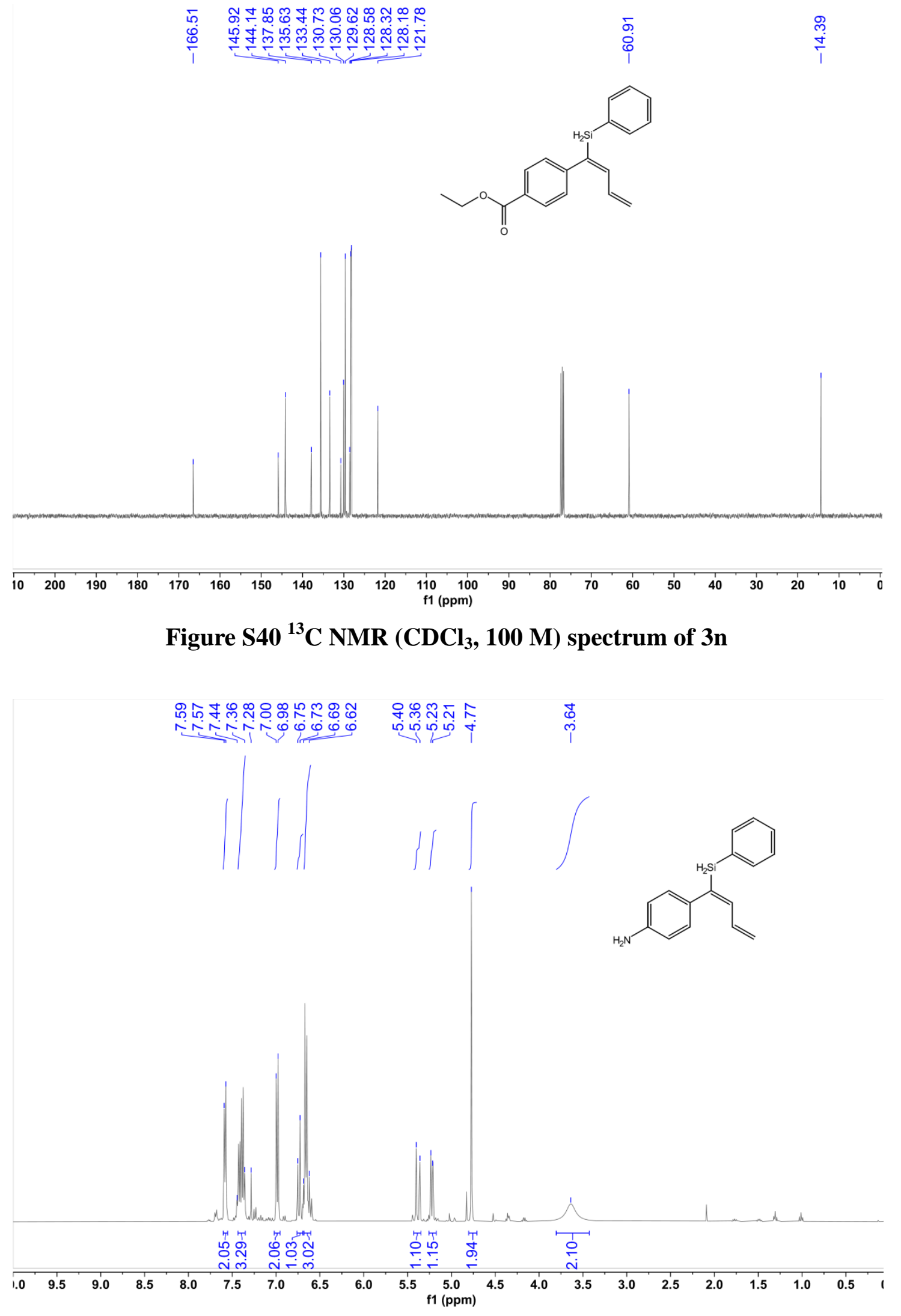

Figure $\mathrm{S41}{ }^{1} \mathrm{H} \mathrm{NMR}\left(\mathrm{CDCl}_{3}, 400 \mathrm{M}\right)$ spectrum of $3 \mathrm{o}$ 

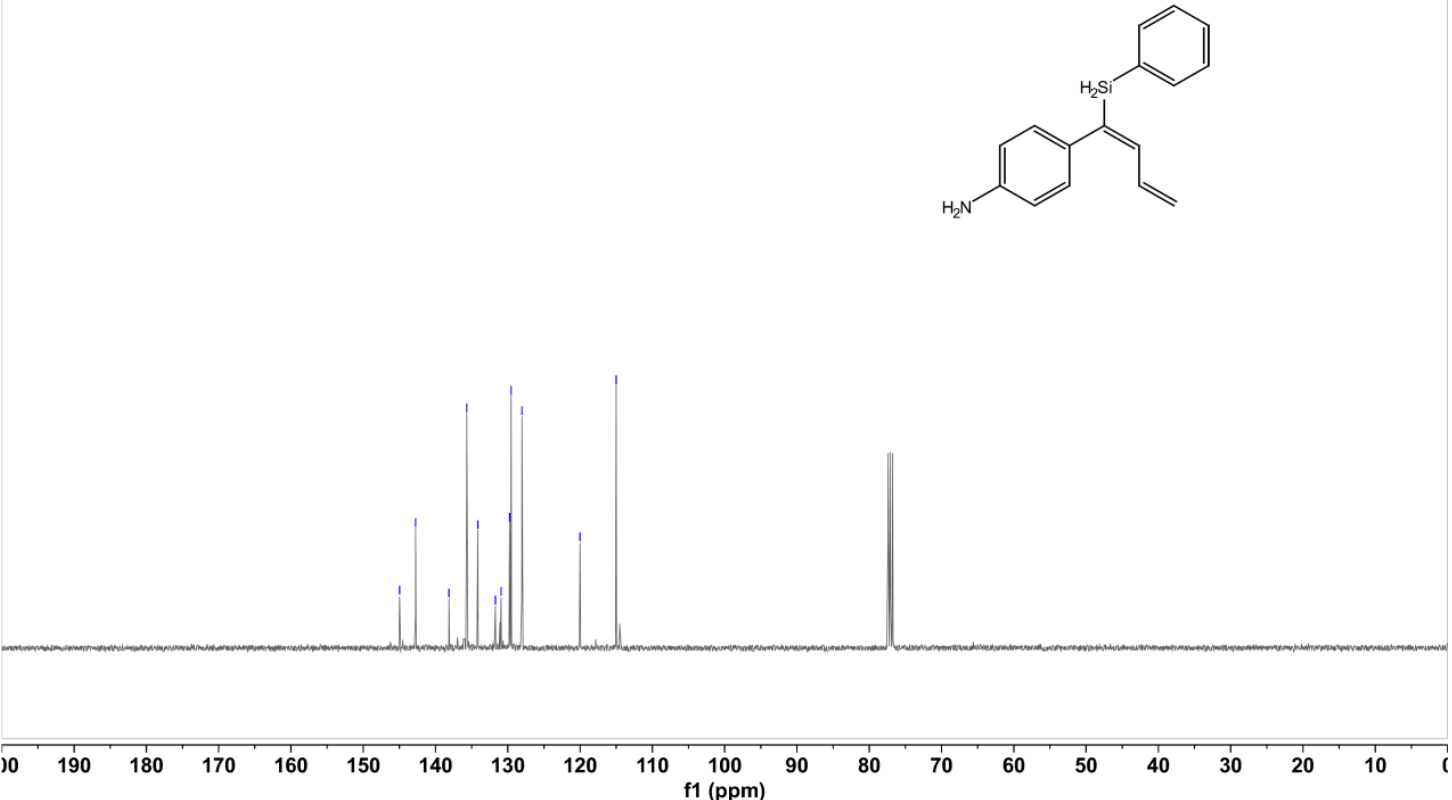

Figure $\mathrm{S42}{ }^{13} \mathrm{C} \mathrm{NMR}\left(\mathrm{CDCl}_{3}, 100 \mathrm{M}\right)$ spectrum of 30

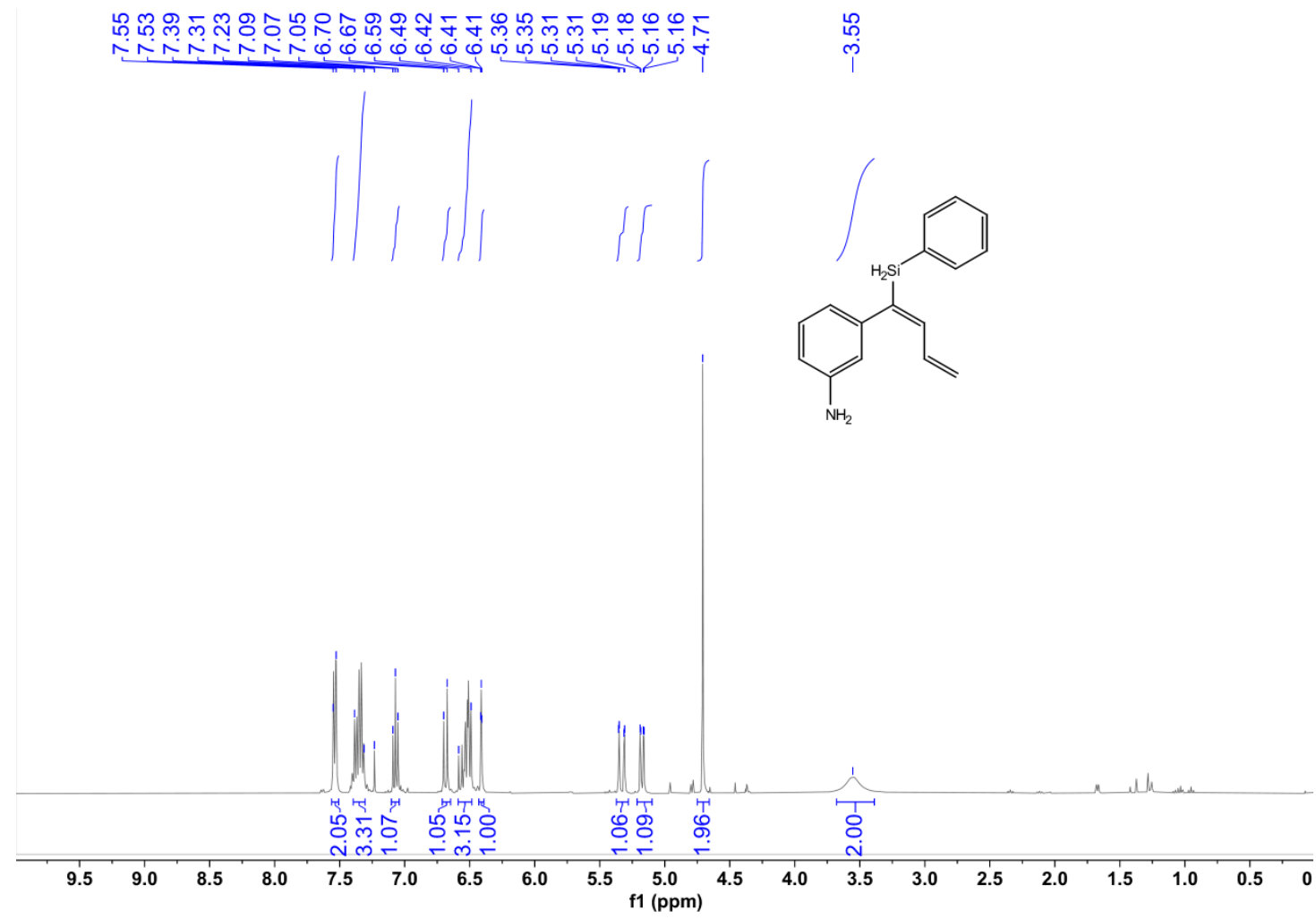

Figure $\mathrm{S} 43{ }^{1} \mathrm{H} \mathrm{NMR}\left(\mathrm{CDCl}_{3}, 400 \mathrm{M}\right)$ spectrum of $3 \mathrm{p}$ 

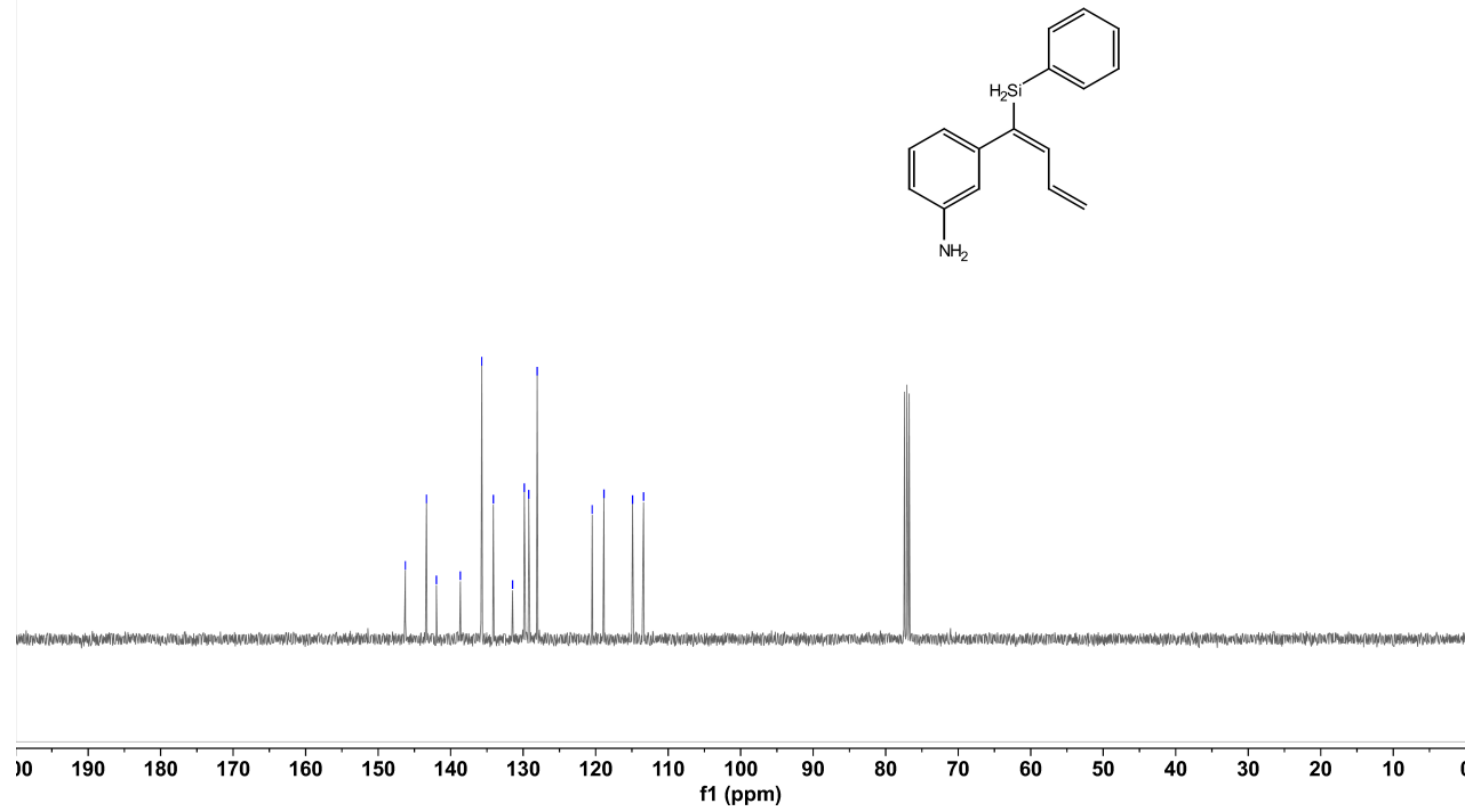

Figure $\mathrm{S44}{ }^{13} \mathrm{C} \mathrm{NMR}\left(\mathrm{CDCl}_{3}, 100 \mathrm{M}\right)$ spectrum of $3 \mathrm{p}$

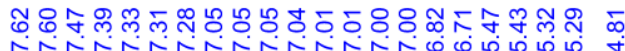

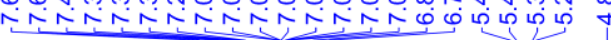
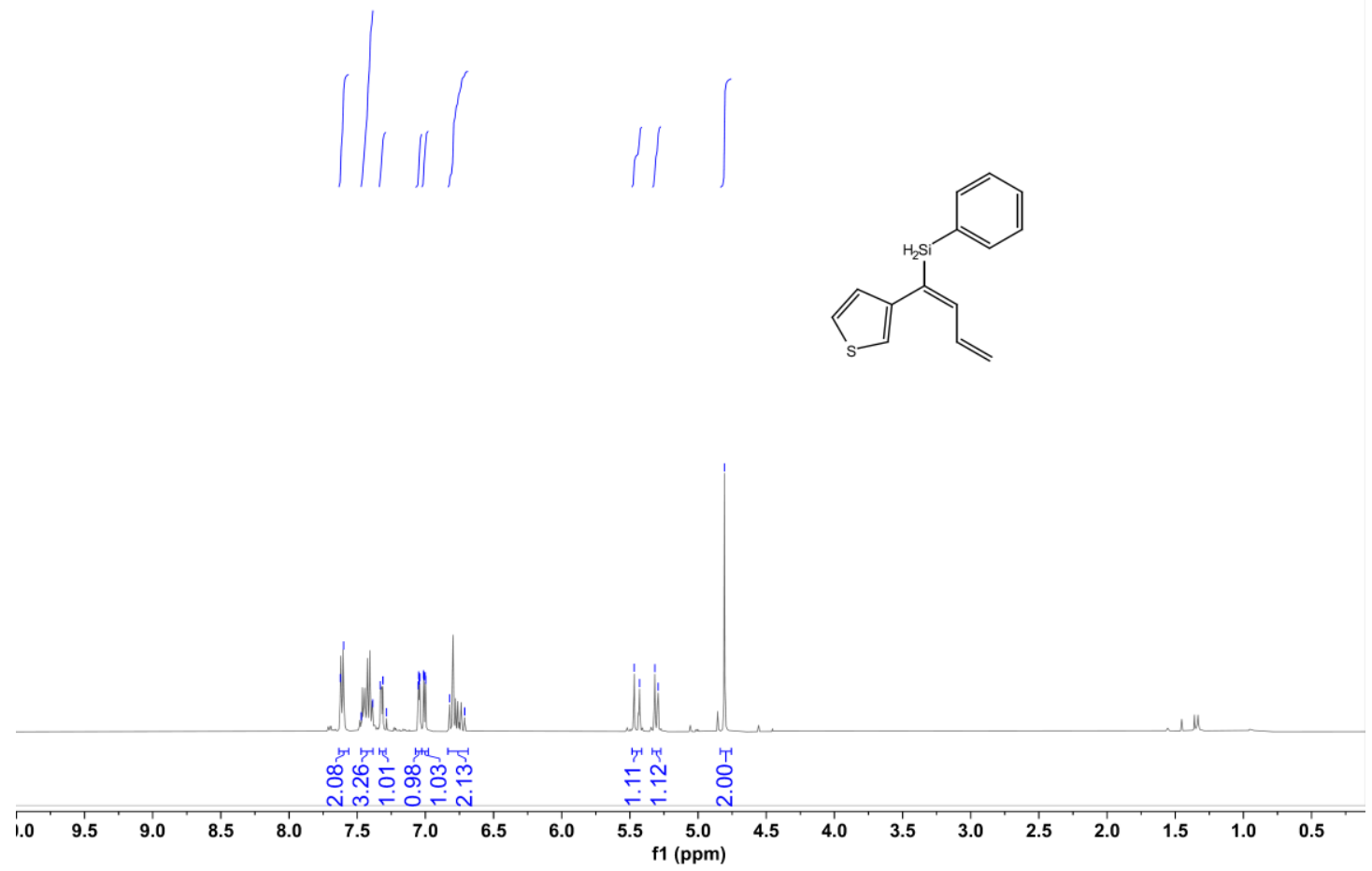

Figure $\mathrm{S45}{ }^{1} \mathrm{H}$ NMR $\left(\mathrm{CDCl}_{3}, 400 \mathrm{M}\right)$ spectrum of $3 \mathrm{q}$ 

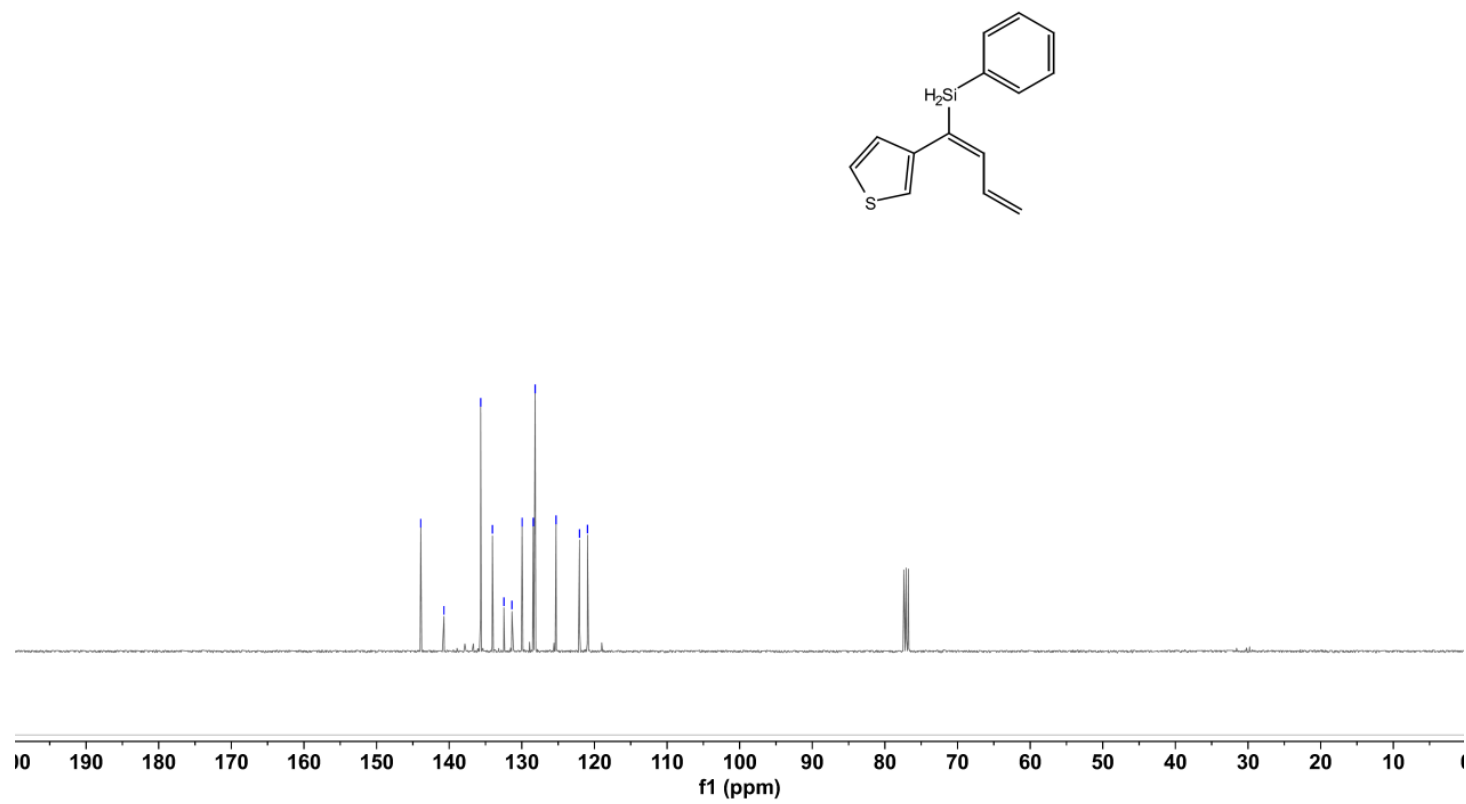

Figure $\mathrm{S46}^{13} \mathrm{C} \mathrm{NMR}\left(\mathrm{CDCl}_{3}, 100 \mathrm{M}\right)$ spectrum of $3 q$

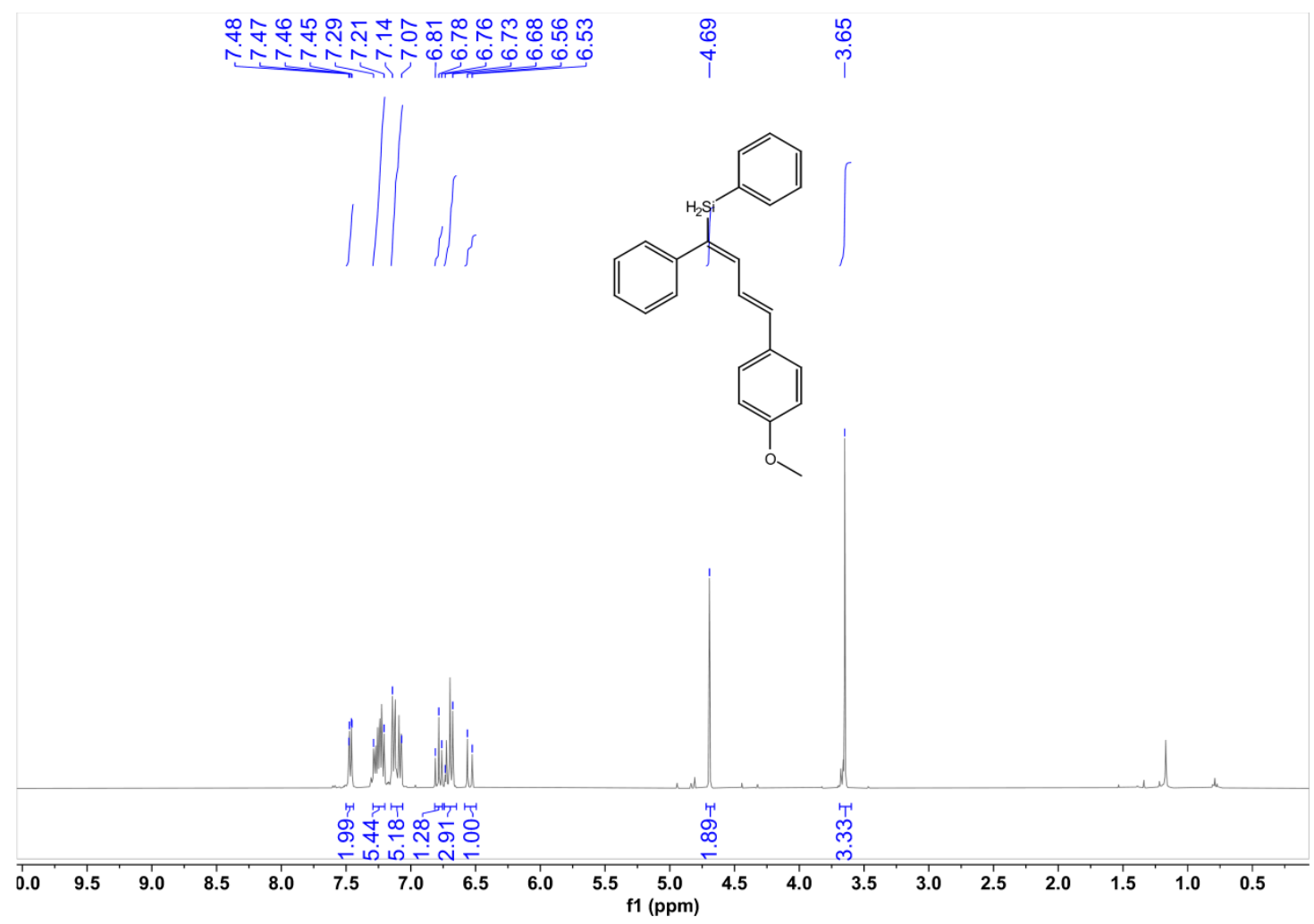

Figure $\mathrm{S} 47{ }^{1} \mathrm{H}$ NMR $\left(\mathrm{CDCl}_{3}, 400 \mathrm{M}\right)$ spectrum of $3 \mathrm{r}$ 
<smiles>COc1ccc(/C=C/C=C(/Nc2ccccc2)c2ccccc2)cc1</smiles>

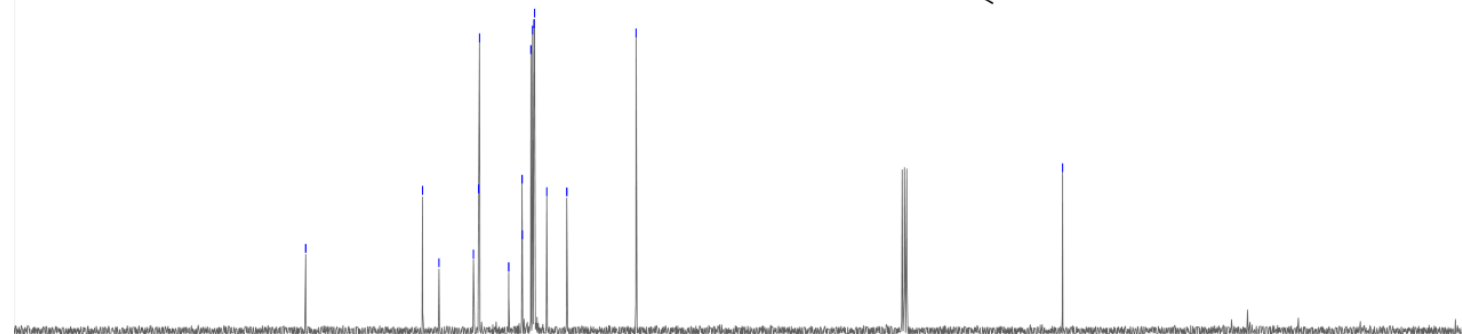

$\begin{array}{llllllllllllllllllllll}10 & 190 & 180 & 170 & 160 & 150 & 140 & 130 & 120 & 110 \begin{array}{c}100 \\ \mathrm{f} 1(\mathrm{ppm})\end{array} & 90 & 80 & 70 & 60 & 50 & 40 & 30 & 20 & 10 & 1\end{array}$

Figure $\mathrm{S}_{4}{ }^{13} \mathrm{C}$ NMR $\left(\mathrm{CDCl}_{3}, 100 \mathrm{M}\right)$ spectrum of $3 \mathrm{r}$

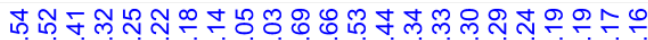

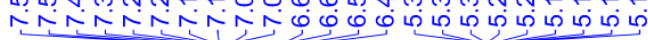
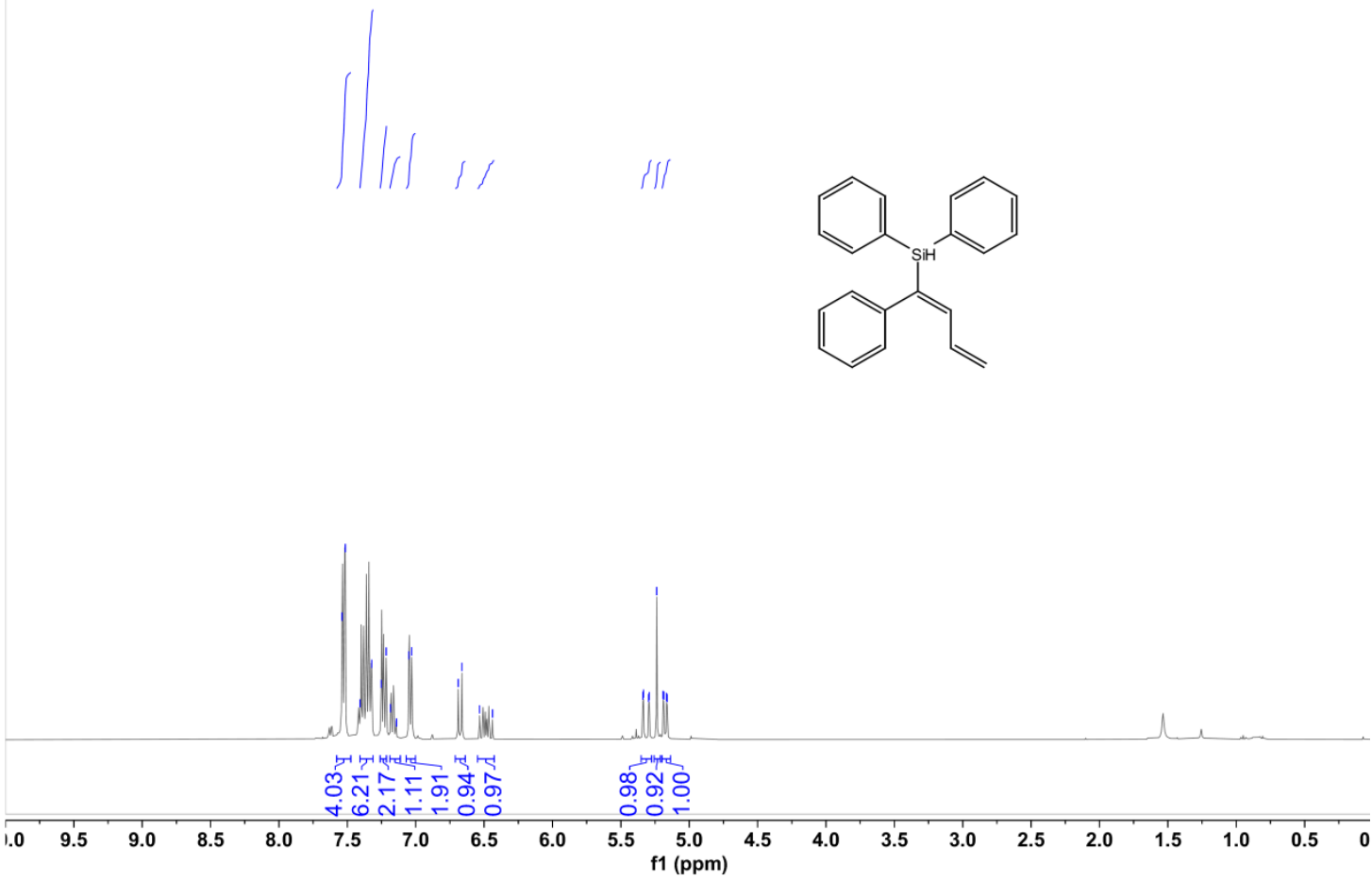

Figure $\mathrm{S49}{ }^{1} \mathrm{H}$ NMR $\left(\mathrm{CDCl}_{3}, 400 \mathrm{M}\right)$ spectrum of $3 \mathrm{a}^{\prime}$ 


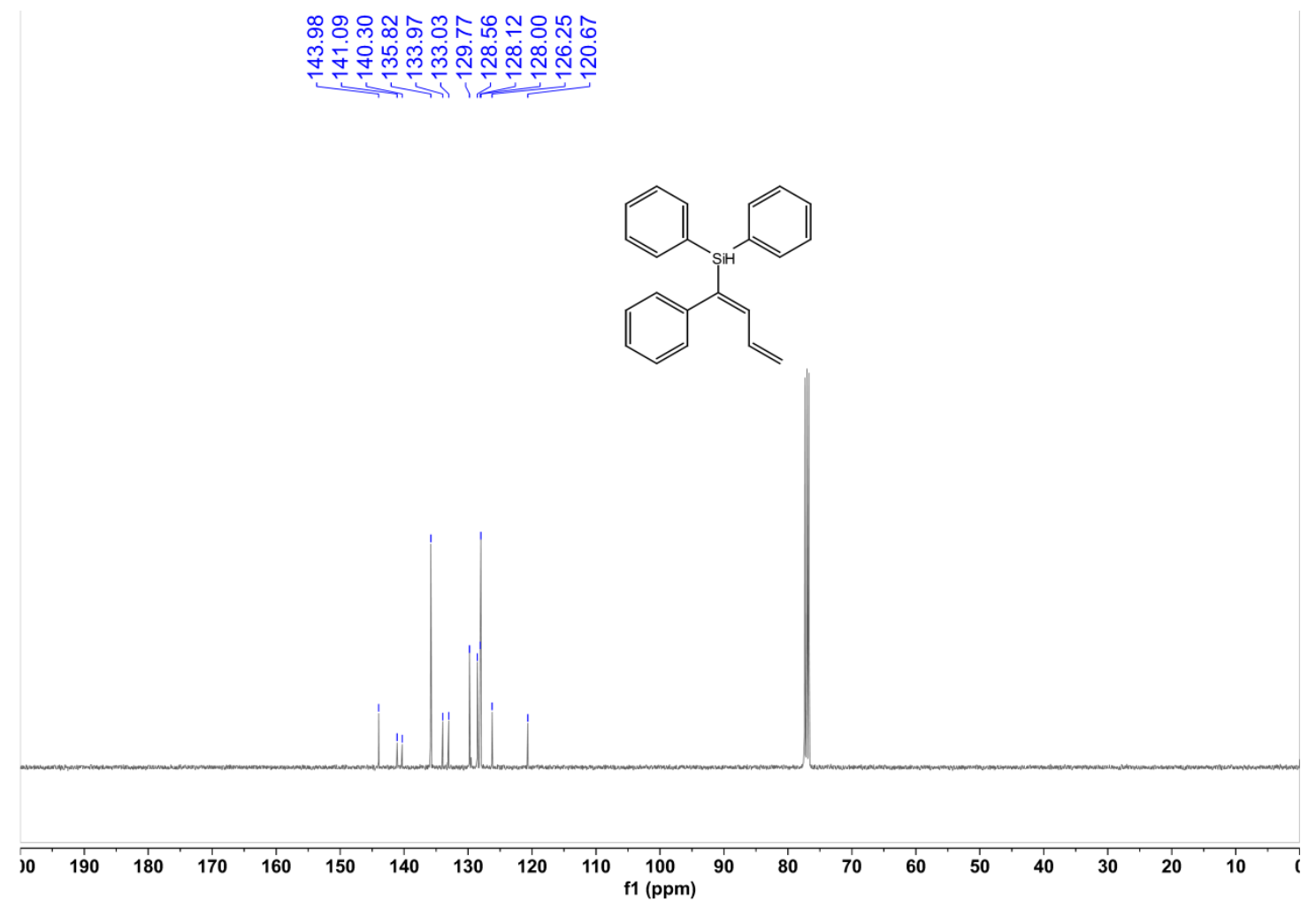

Figure $\mathrm{S50}^{13} \mathrm{C} \mathrm{NMR}\left(\mathrm{CDCl}_{3}, 100 \mathrm{M}\right)$ spectrum of $3 \mathrm{a}^{\prime}$

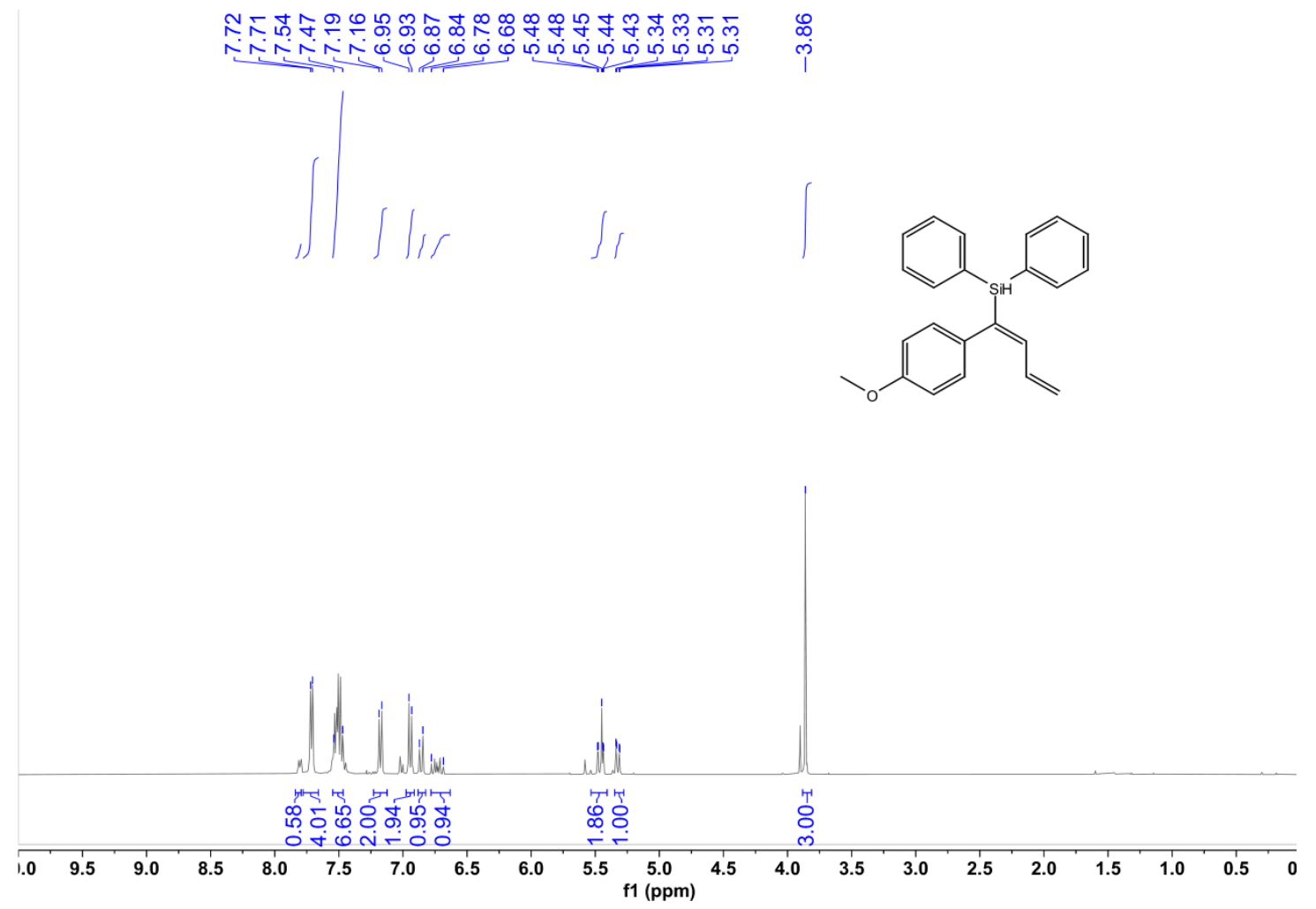

Figure $\mathrm{S51}^{1} \mathrm{H} \mathrm{NMR}\left(\mathrm{CDCl}_{3}, 400 \mathrm{M}\right)$ spectrum of 3d' 


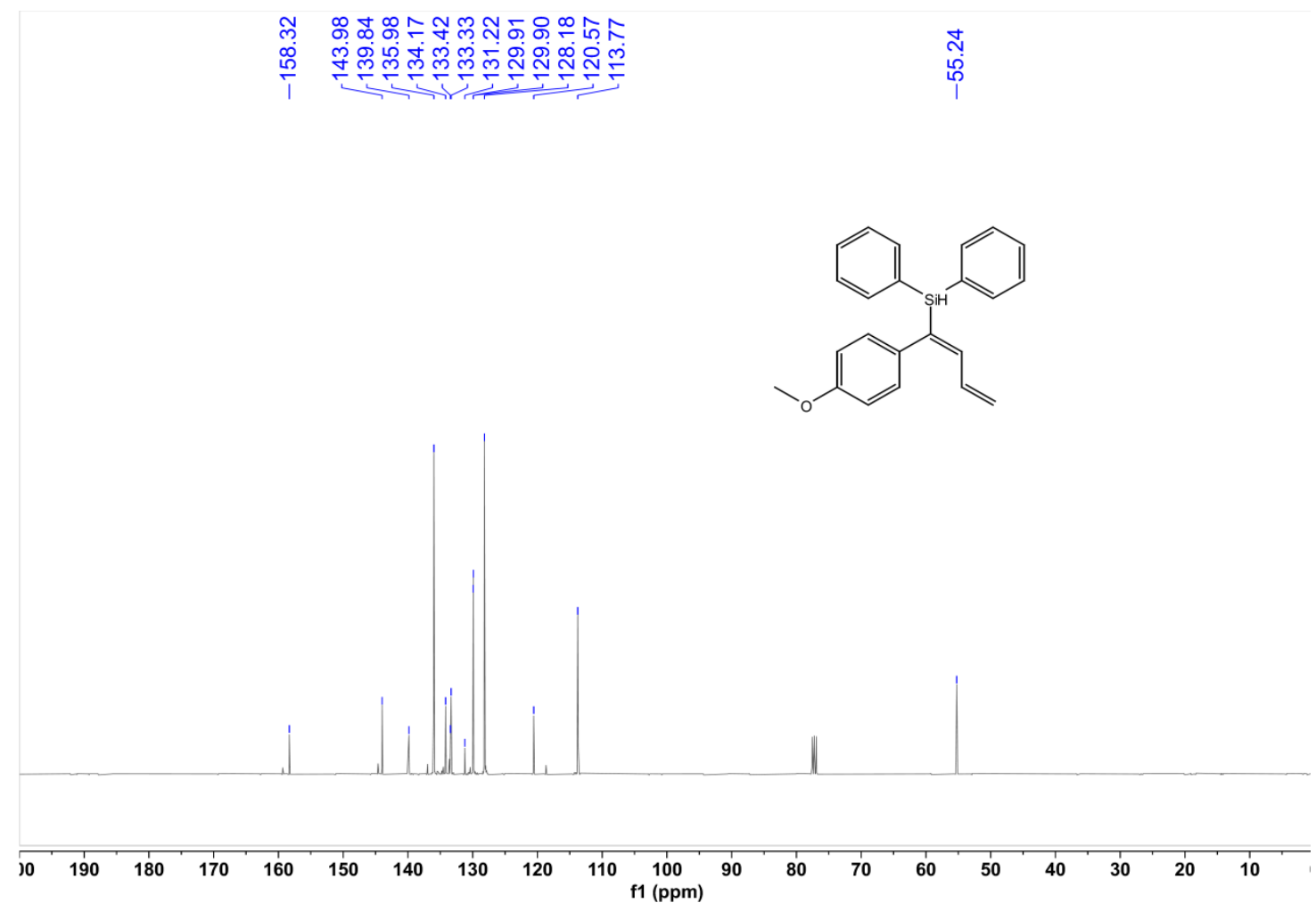

Figure $\mathrm{S52}^{13} \mathrm{C} \mathrm{NMR}\left(\mathrm{CDCl}_{3}, 100 \mathrm{M}\right)$ spectrum of 3d'

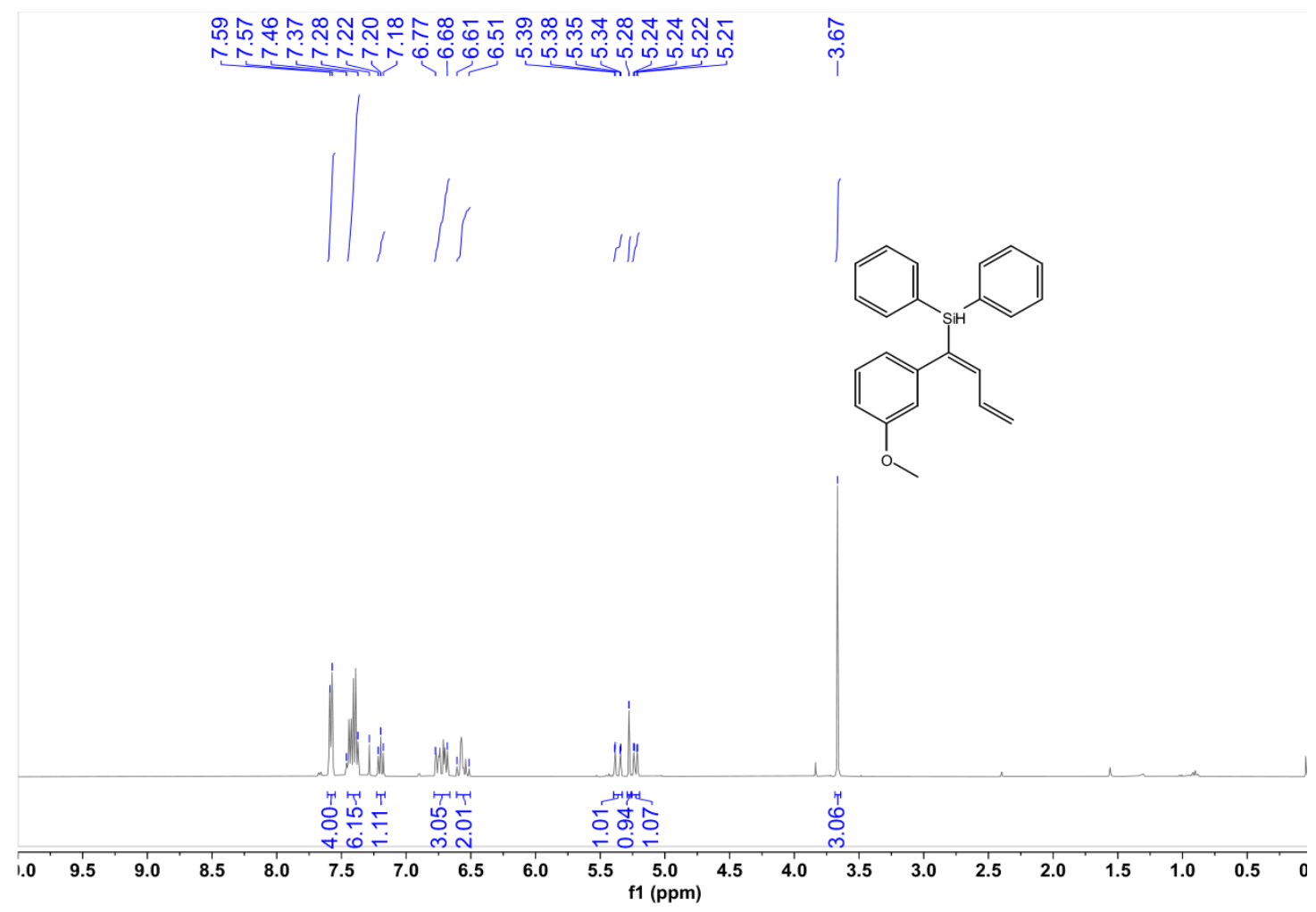

Figure $\mathrm{S53}^{1} \mathrm{H}$ NMR $\left(\mathrm{CDCl}_{3}, 400 \mathrm{M}\right)$ spectrum of $3 \mathrm{e}^{\prime}$ 

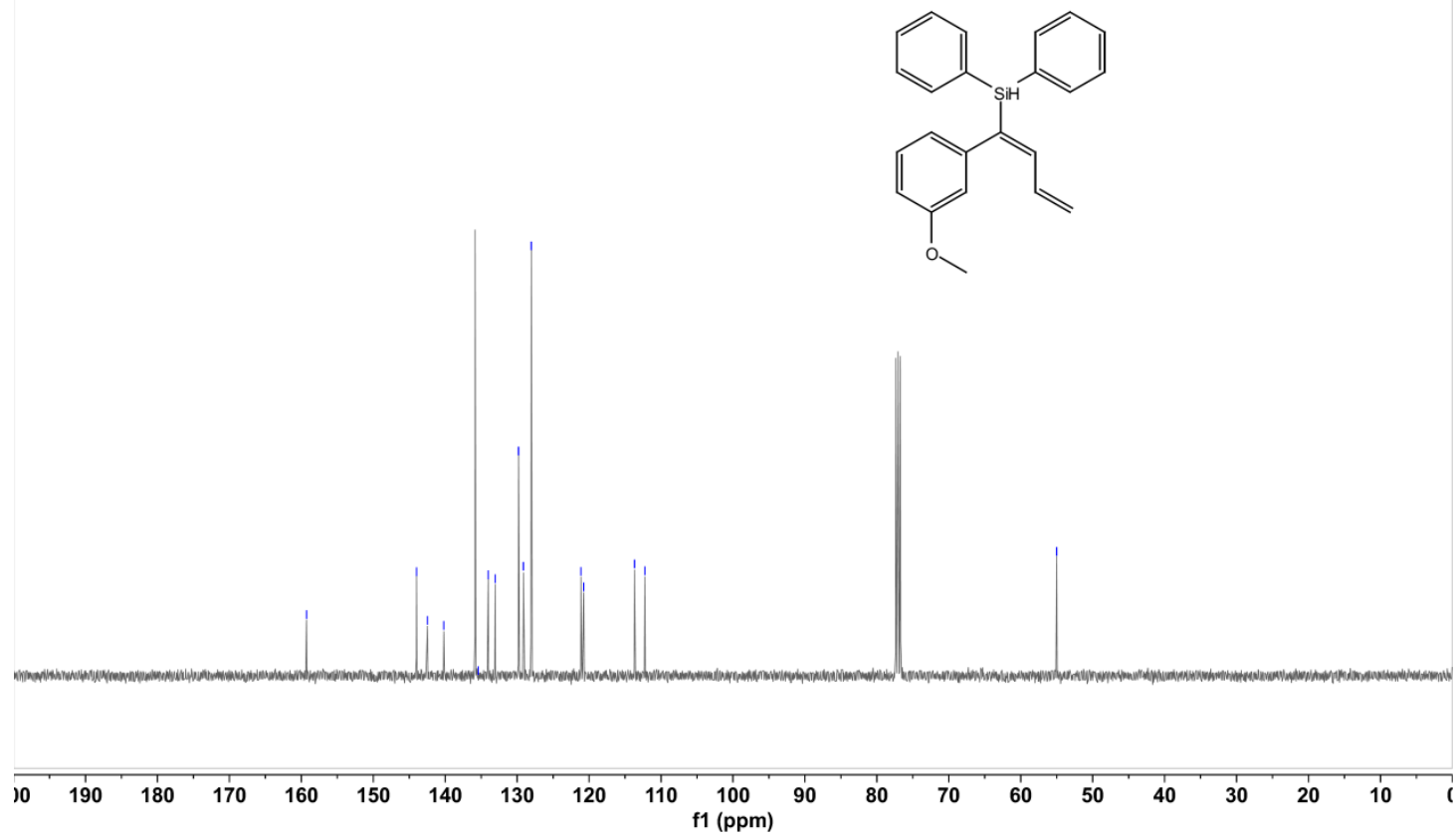

Figure $554{ }^{13} \mathrm{C} \mathrm{NMR}\left(\mathrm{CDCl}_{3}, 100 \mathrm{M}\right)$ spectrum of $3 \mathrm{e}^{\prime}$

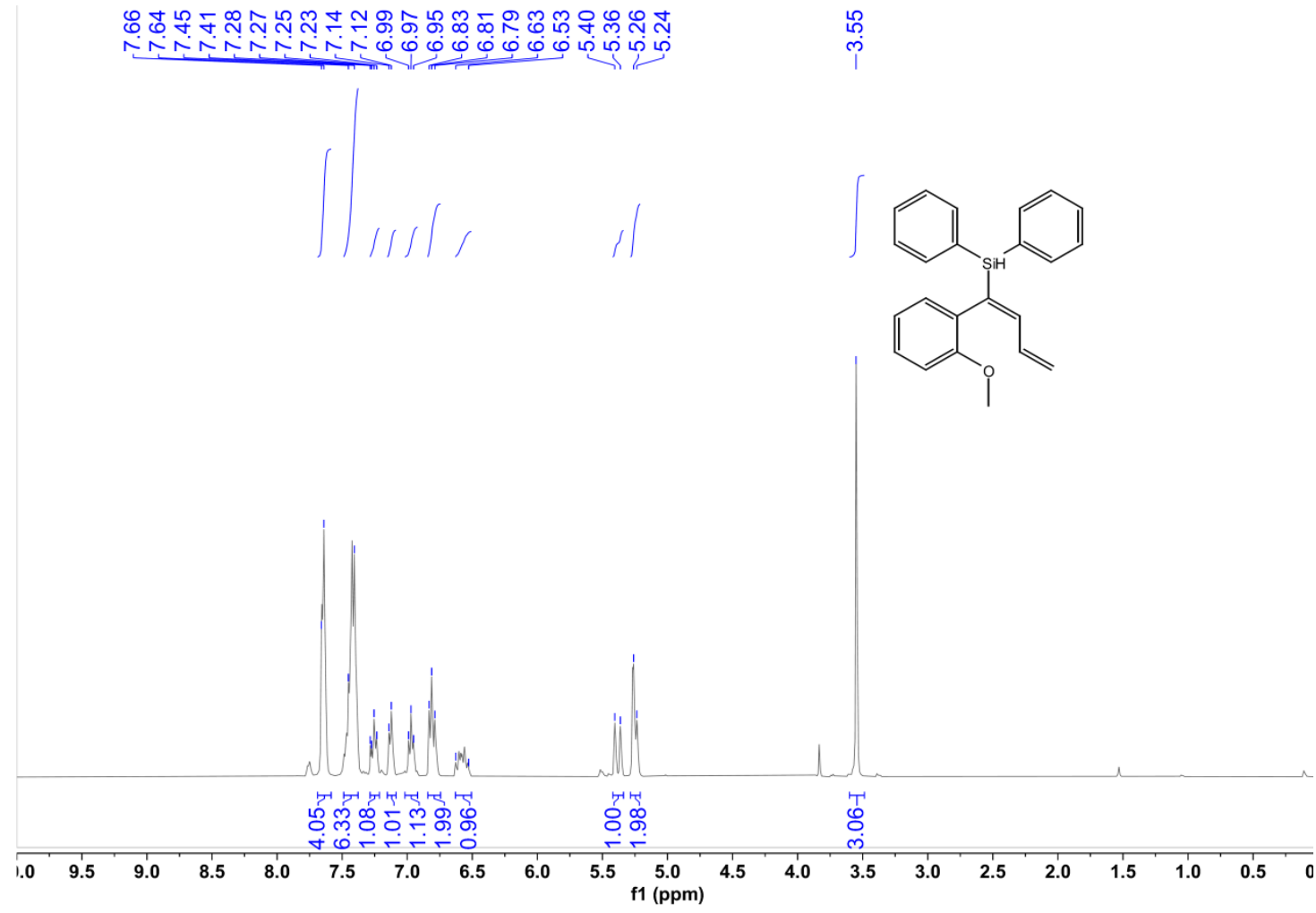

Figure $\mathrm{S55}^{1} \mathrm{H} \mathrm{NMR}\left(\mathrm{CDCl}_{3}, 400 \mathrm{M}\right)$ spectrum of $3 \mathrm{f}^{\prime}$ 

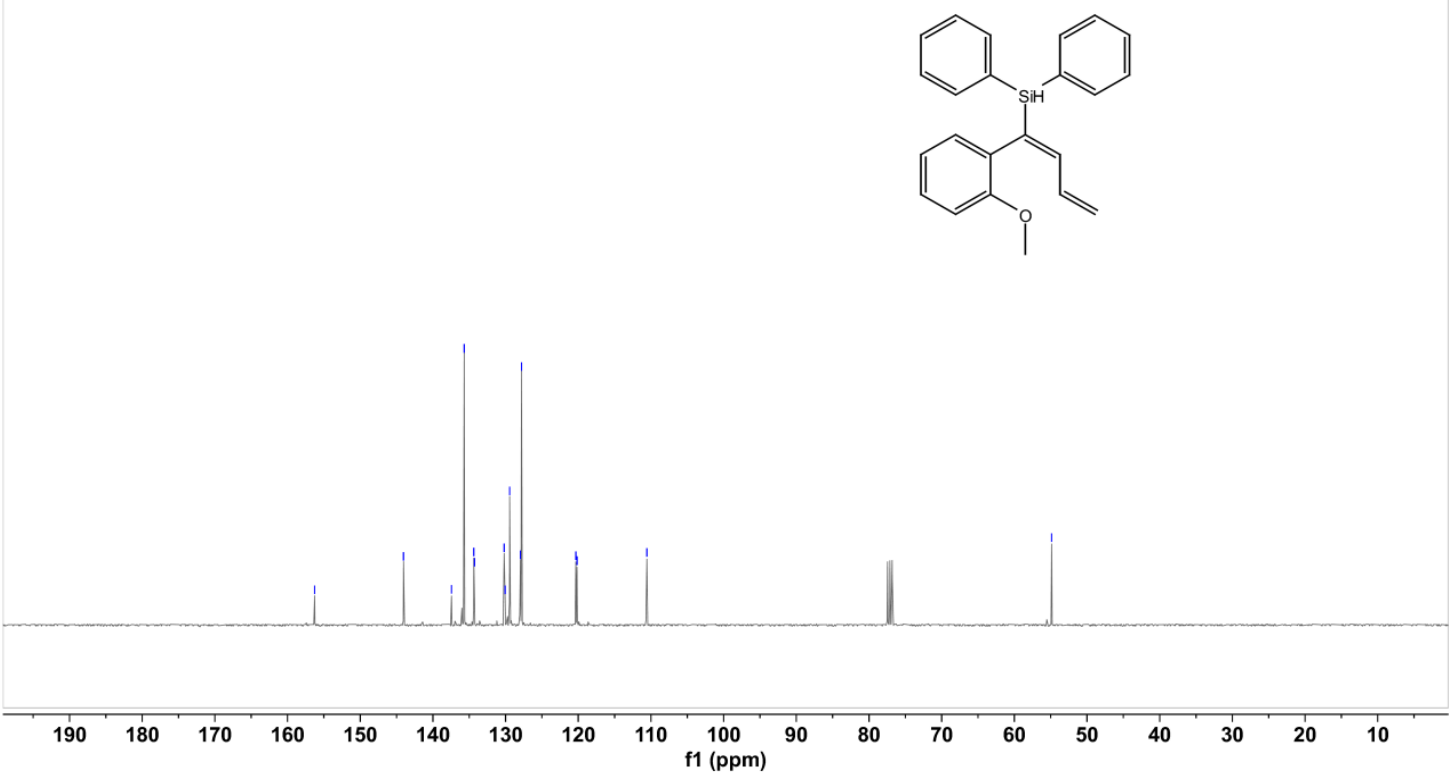

Figure $\mathrm{S56}^{13} \mathrm{C} \mathrm{NMR}\left(\mathrm{CDCl}_{3}, 100 \mathrm{M}\right)$ spectrum of $3 \mathrm{f}^{\prime}$

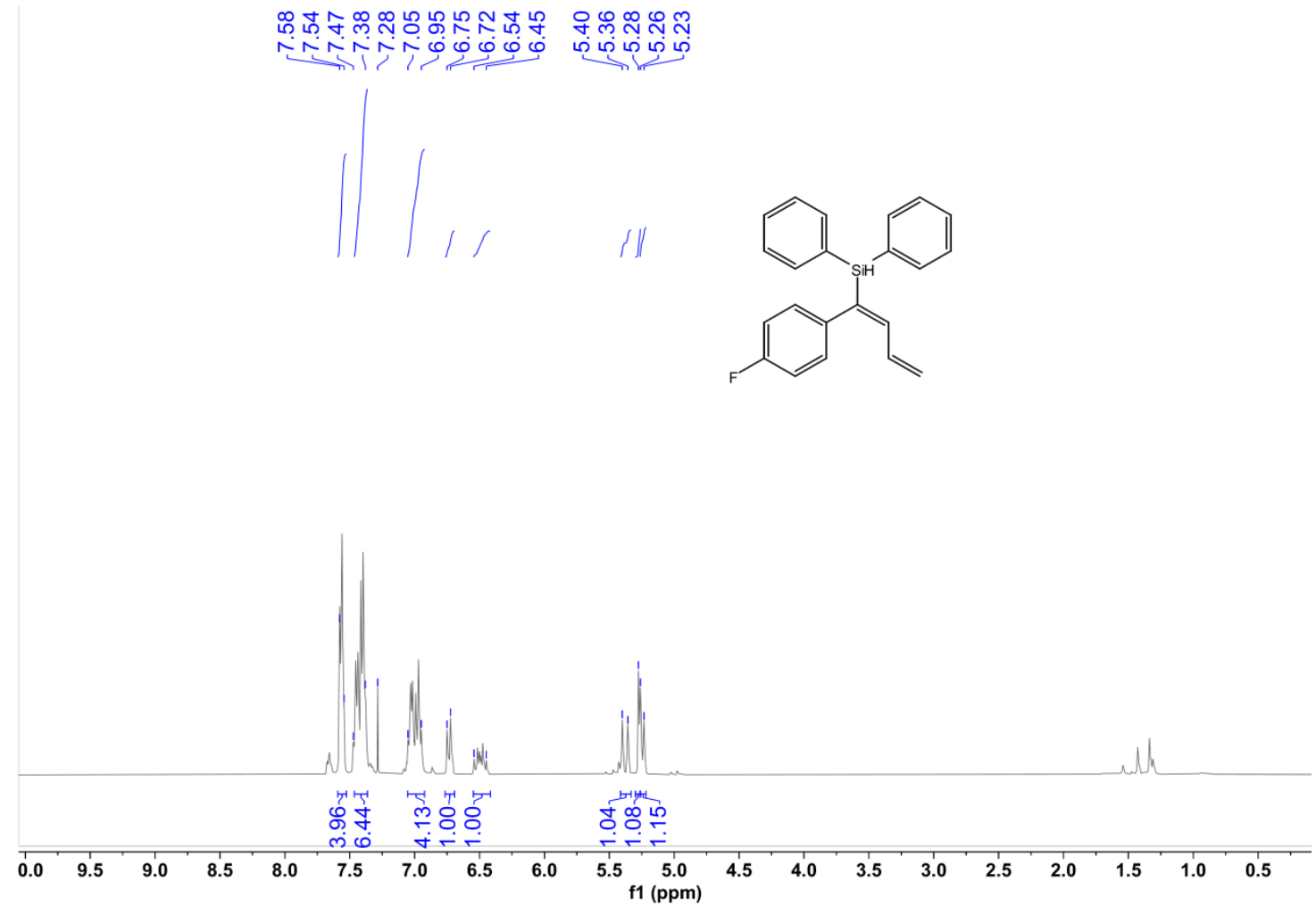

Figure S57 ${ }^{1} \mathrm{H}$ NMR $\left(\mathrm{CDCl}_{3}, 400 \mathrm{M}\right)$ spectrum of $3 \mathrm{~h}^{\prime}$ 


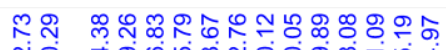

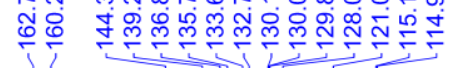
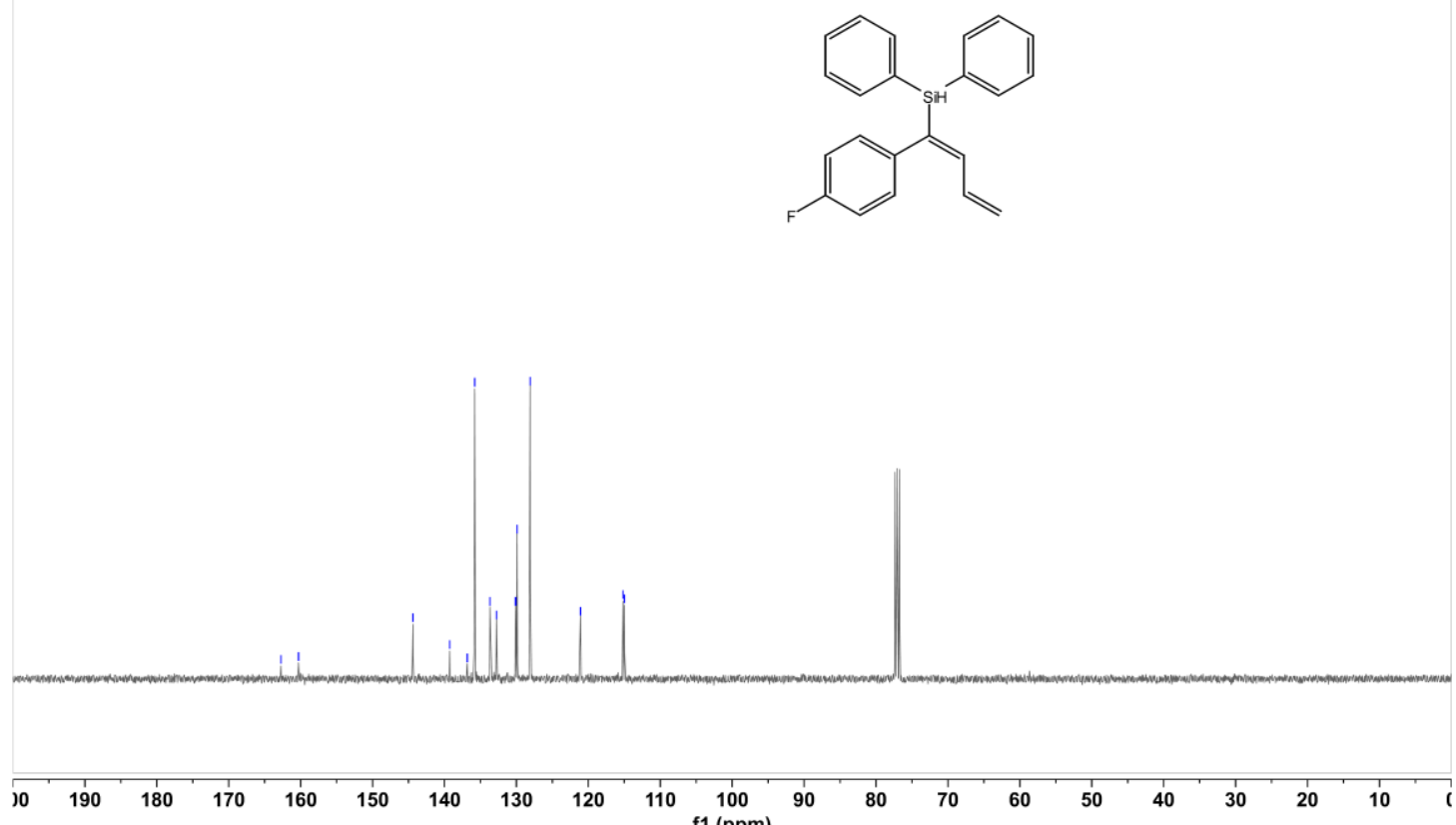

Figure $\mathrm{S58}^{13} \mathrm{C} \mathrm{NMR}\left(\mathrm{CDCl}_{3}, 100 \mathrm{M}\right)$ spectrum of $3 \mathrm{~h}^{\prime}$

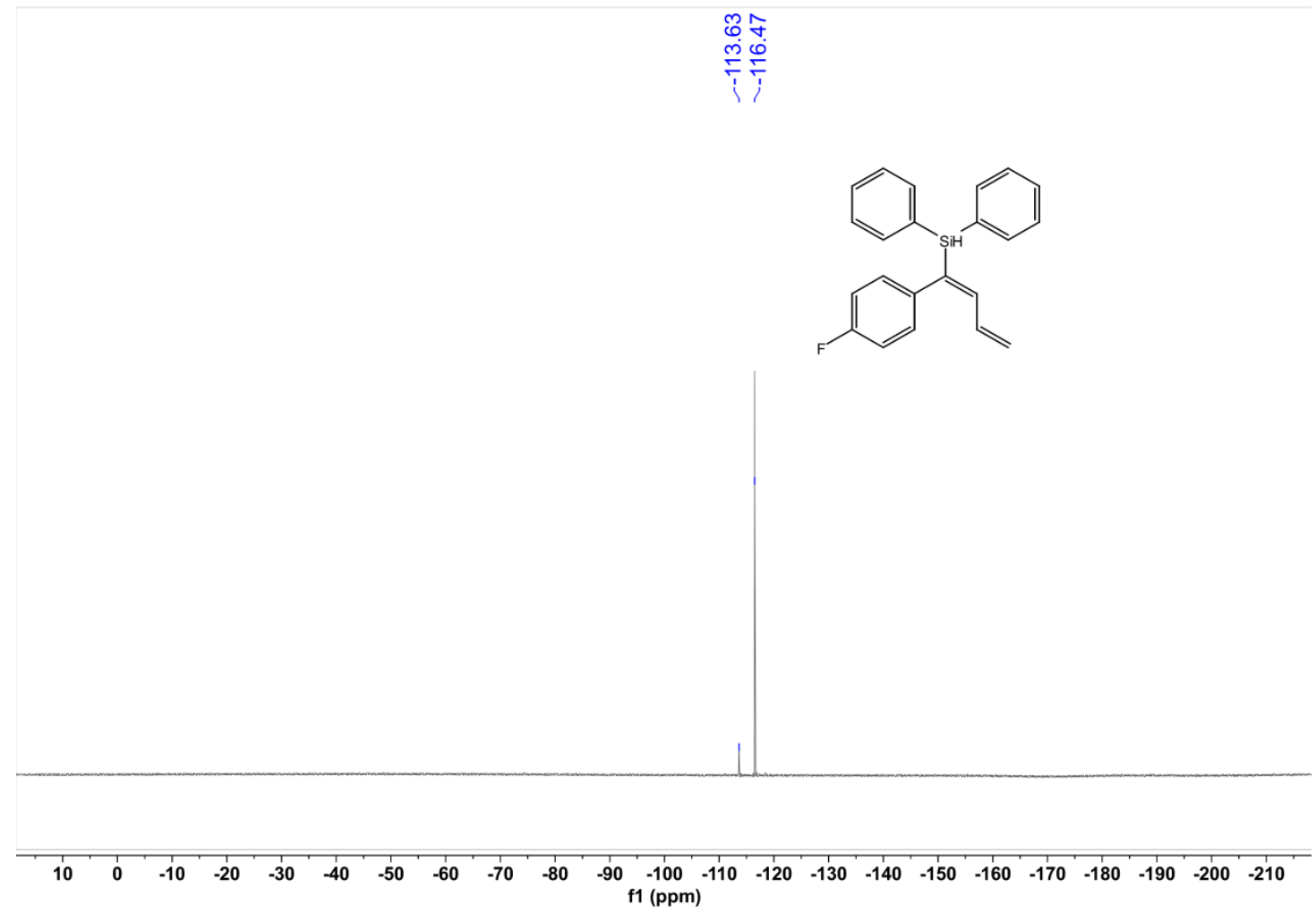

Figure $559{ }^{19} \mathrm{~F}$ NMR $\left(\mathrm{CDCl}_{3}, 376 \mathrm{MHz}\right)$ spectrum of 3h' 


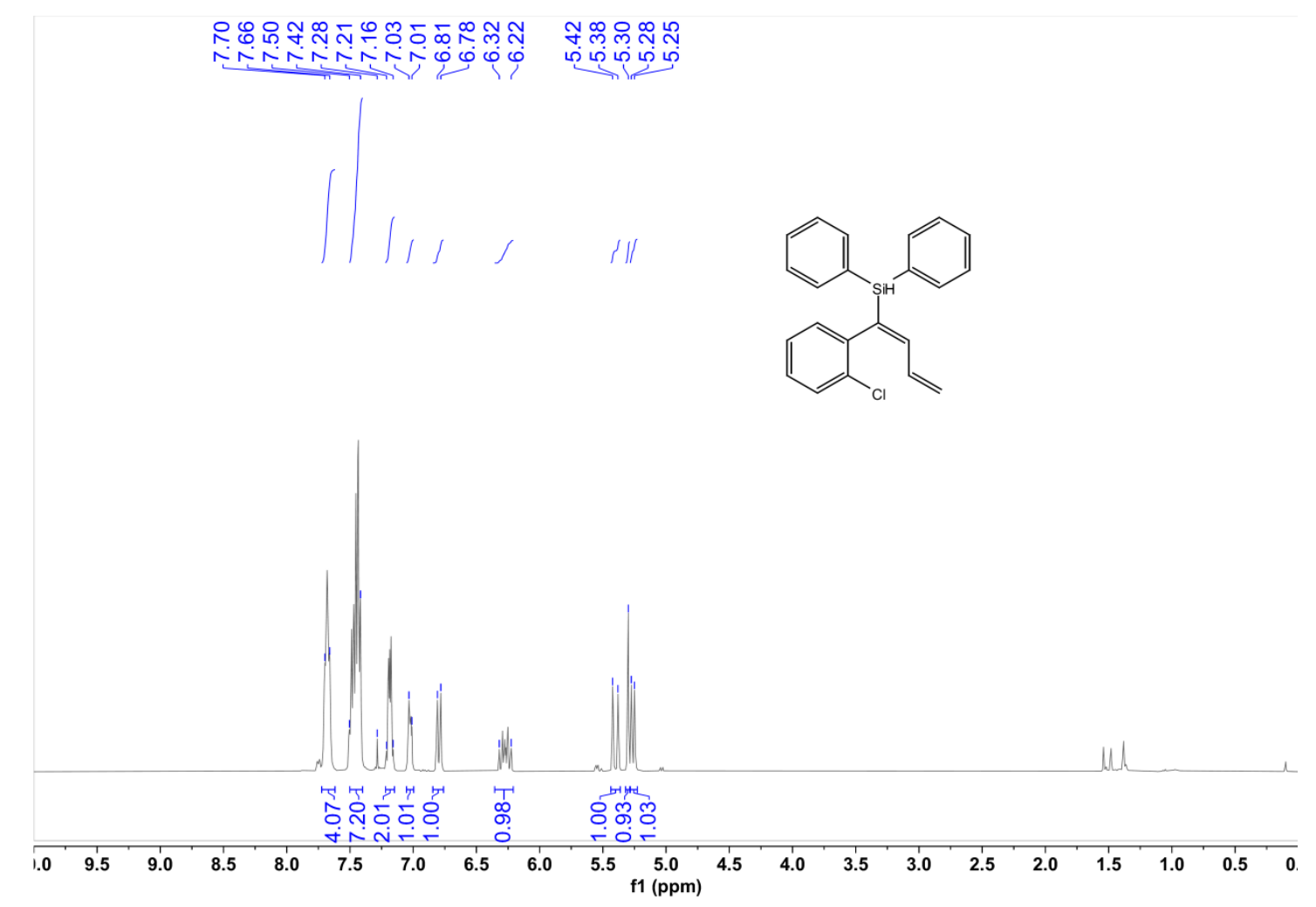

Figure $\mathrm{S60}{ }^{1} \mathrm{H} \mathrm{NMR}\left(\mathrm{CDCl}_{3}, 400 \mathrm{M}\right)$ spectrum of $3 \mathrm{j}$ '
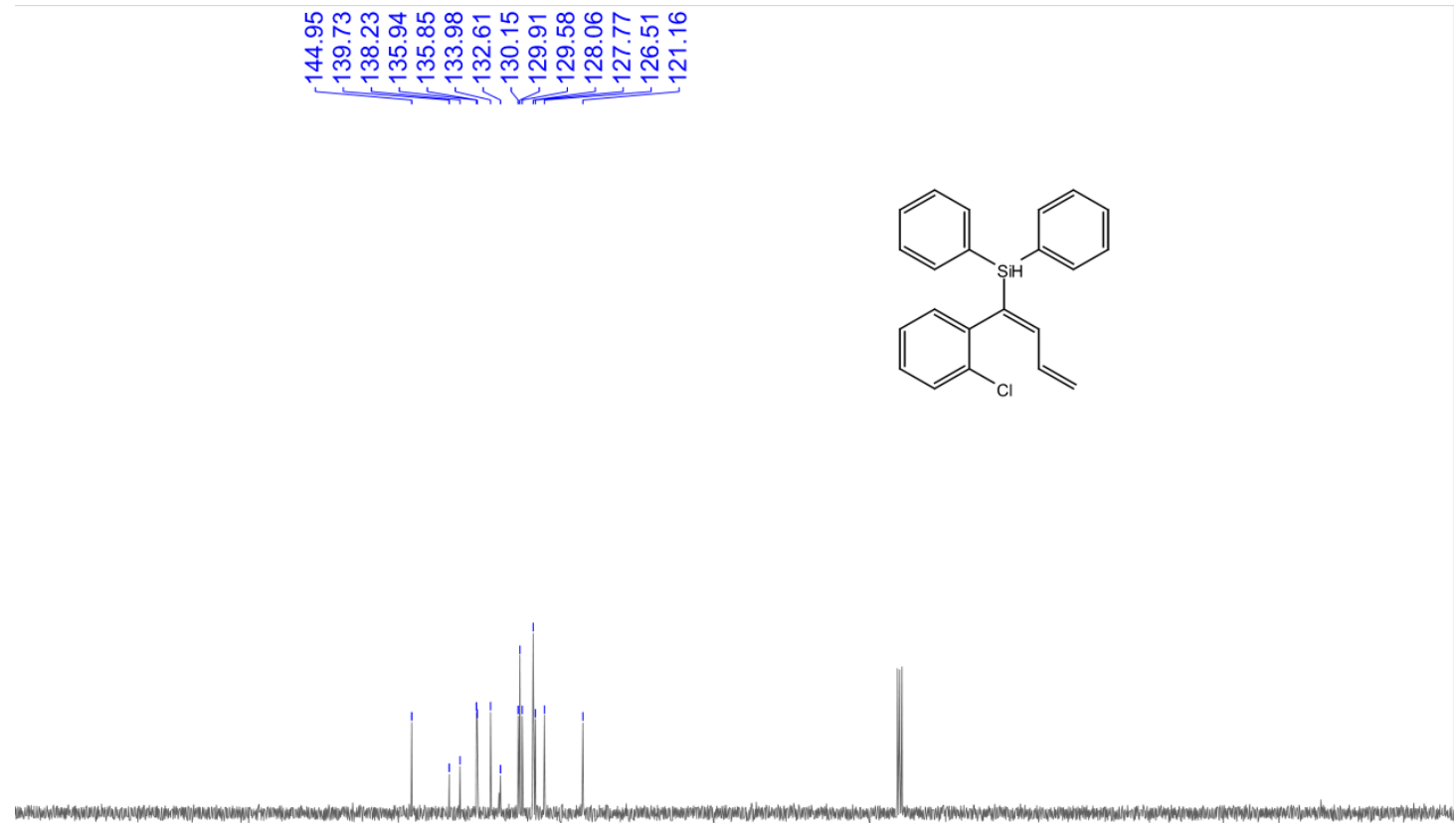

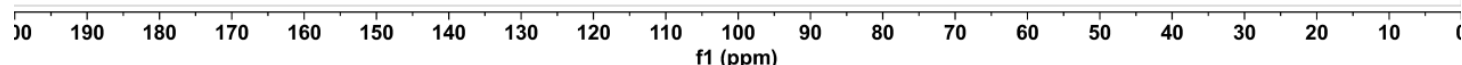

Figure $\mathrm{S}^{13}{ }^{13} \mathrm{C} \mathrm{NMR}\left(\mathrm{CDCl}_{3}, 100 \mathrm{M}\right)$ spectrum of $3 \mathrm{j}$ ' 


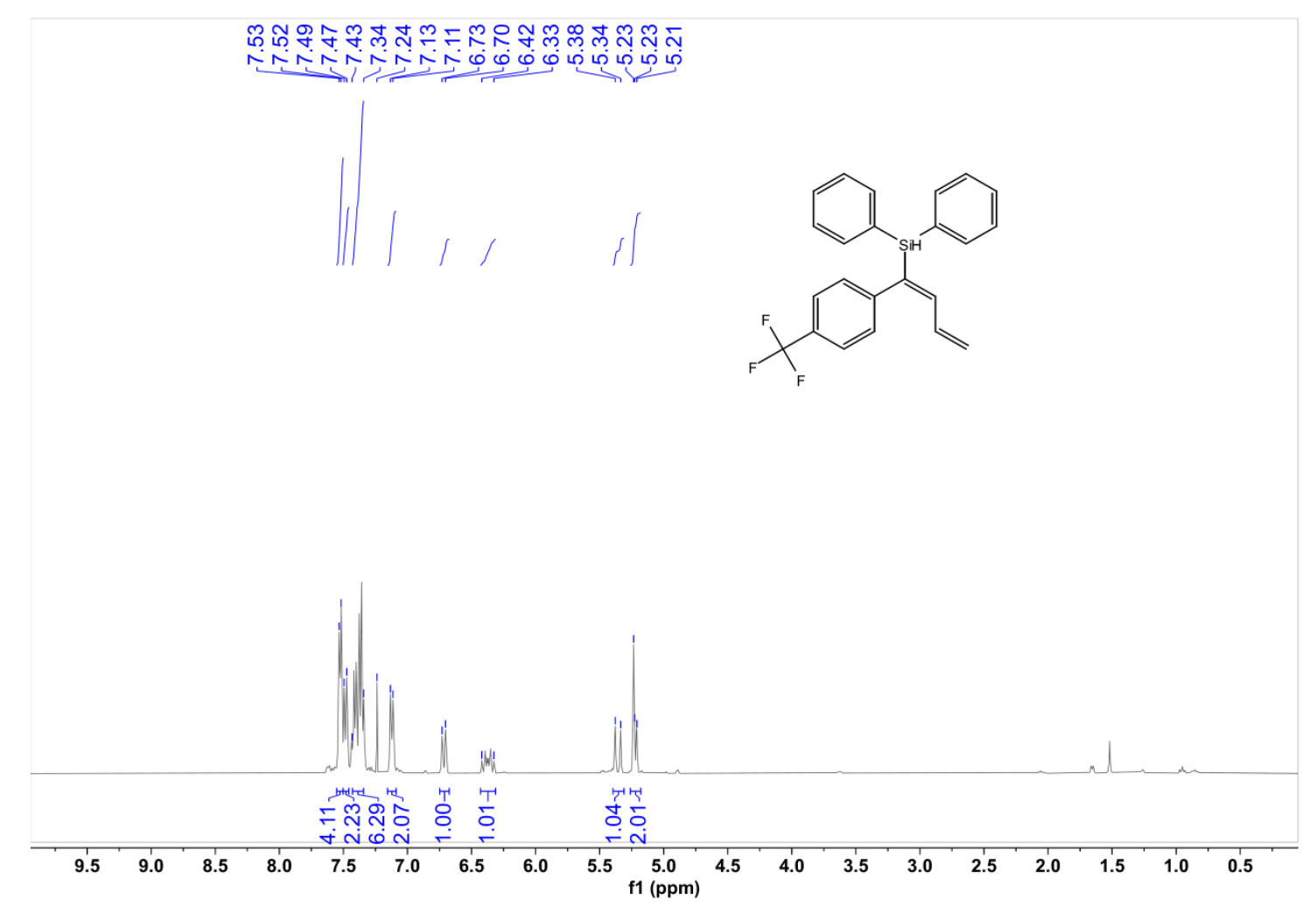

Figure $\mathrm{S62}{ }^{1} \mathrm{H} \mathrm{NMR}\left(\mathrm{CDCl}_{3}, 400 \mathrm{M}\right)$ spectrum of $3 \mathrm{~m}^{\prime}$
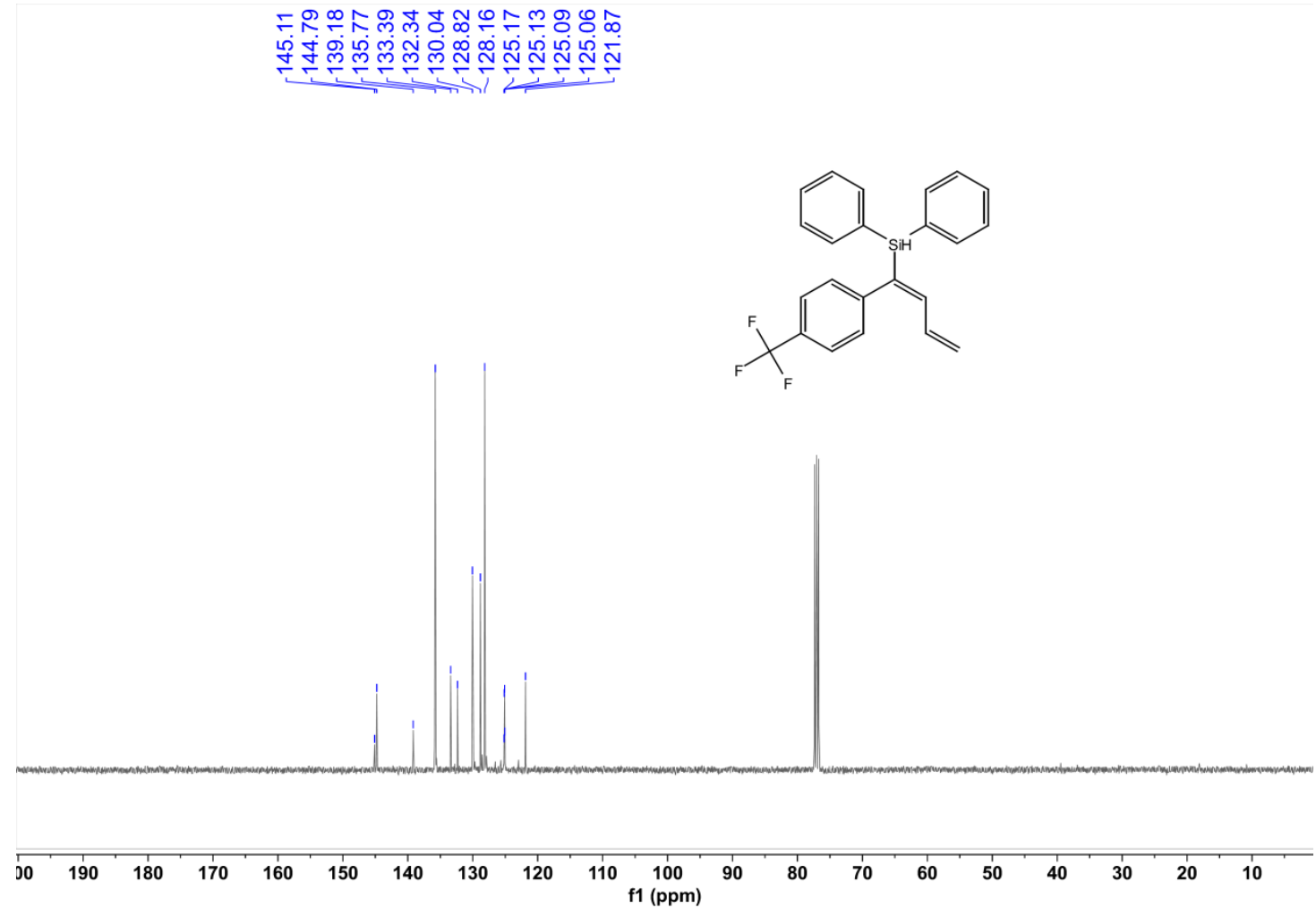

Figure $\mathrm{S} 63{ }^{13} \mathrm{C} \mathrm{NMR}\left(\mathrm{CDCl}_{3}, 100 \mathrm{M}\right)$ spectrum of $3 \mathrm{~m}^{\prime}$ 


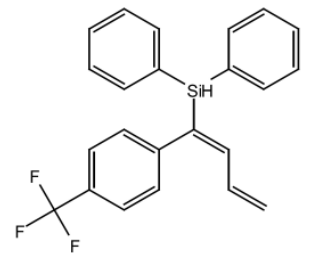

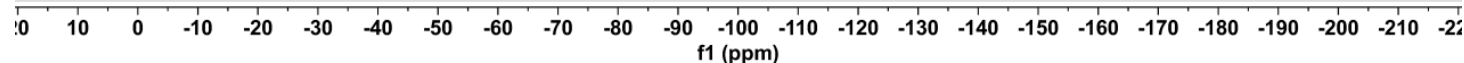

Figure $\mathrm{S} 4{ }^{19} \mathrm{~F} \mathrm{NMR}\left(\mathrm{CDCl}_{3}, 376 \mathrm{MHz}\right)$ spectrum of 3m'

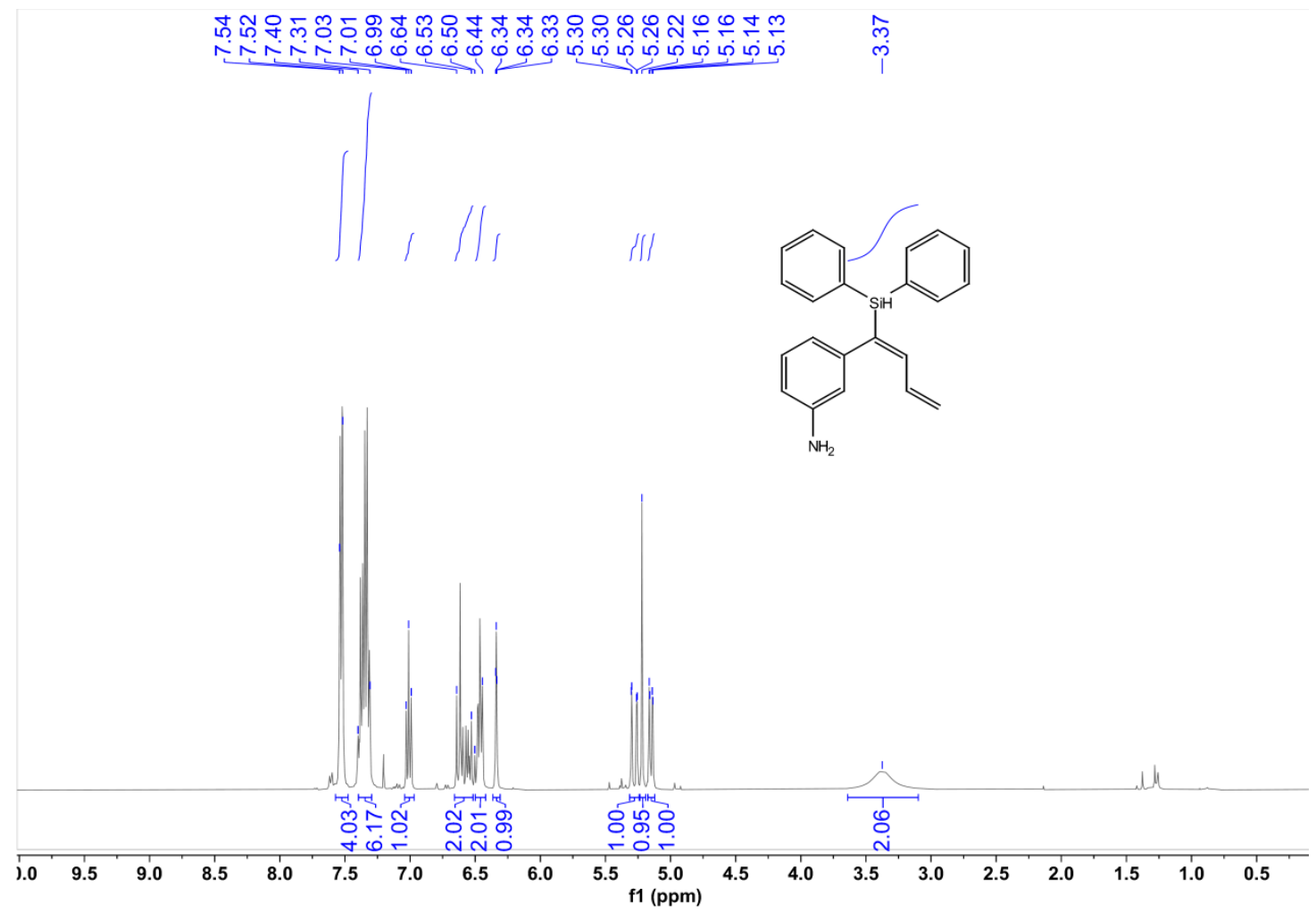

Figure $\mathrm{S}^{2}{ }^{1} \mathrm{H}$ NMR $\left(\mathrm{CDCl}_{3}, 400 \mathrm{M}\right)$ spectrum of $3 \mathrm{p}^{\prime}$ 

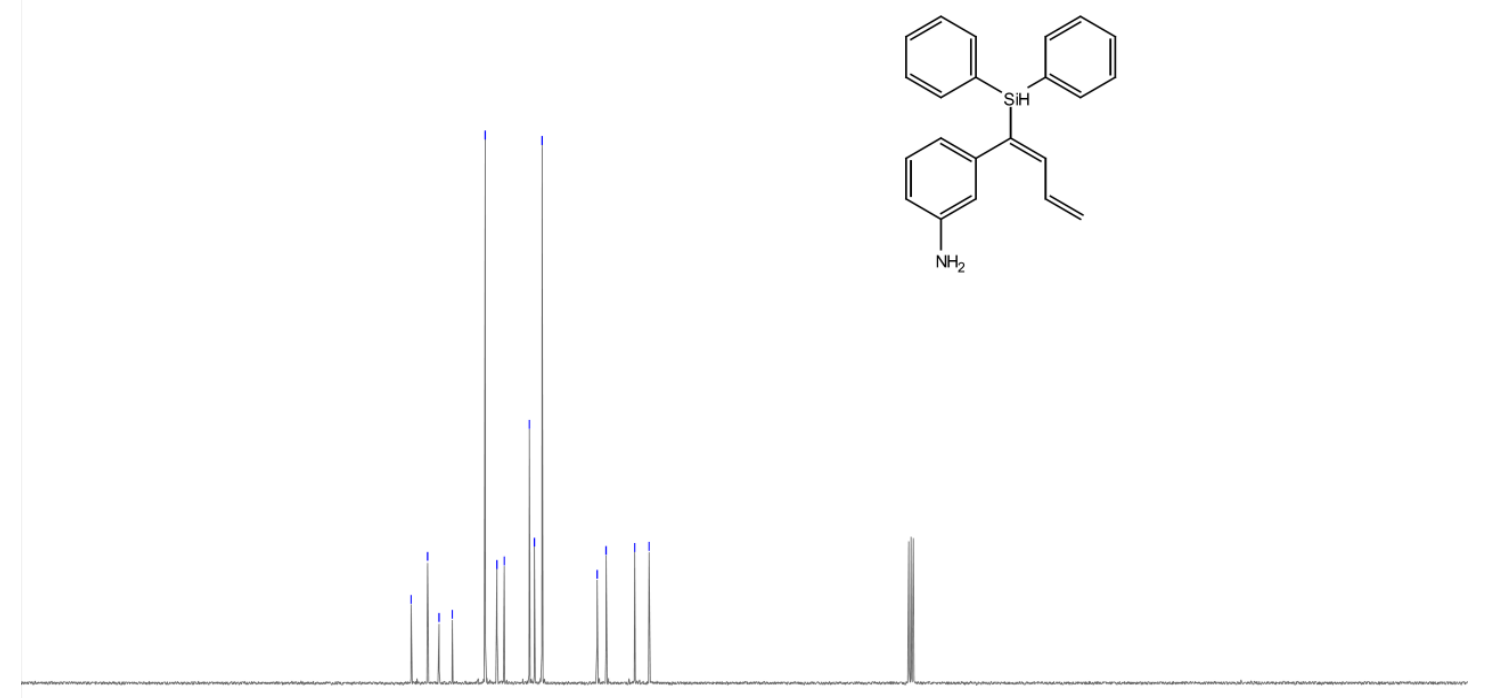

\begin{tabular}{llllllllllllllllllllll}
\hline 10 & 190 & 180 & 170 & 160 & 150 & 140 & 130 & 120 & 110 & $\begin{array}{c}100 \\
\mathrm{f} 1(\mathrm{ppm})\end{array}$ & 90 & 80 & 70 & 60 & 50 & 40 & 30 & 20 & 10 & 1
\end{tabular}

Figure $\mathrm{S66}{ }^{13} \mathrm{C} \mathrm{NMR}\left(\mathrm{CDCl}_{3}, 100 \mathrm{M}\right)$ spectrum of 3p'

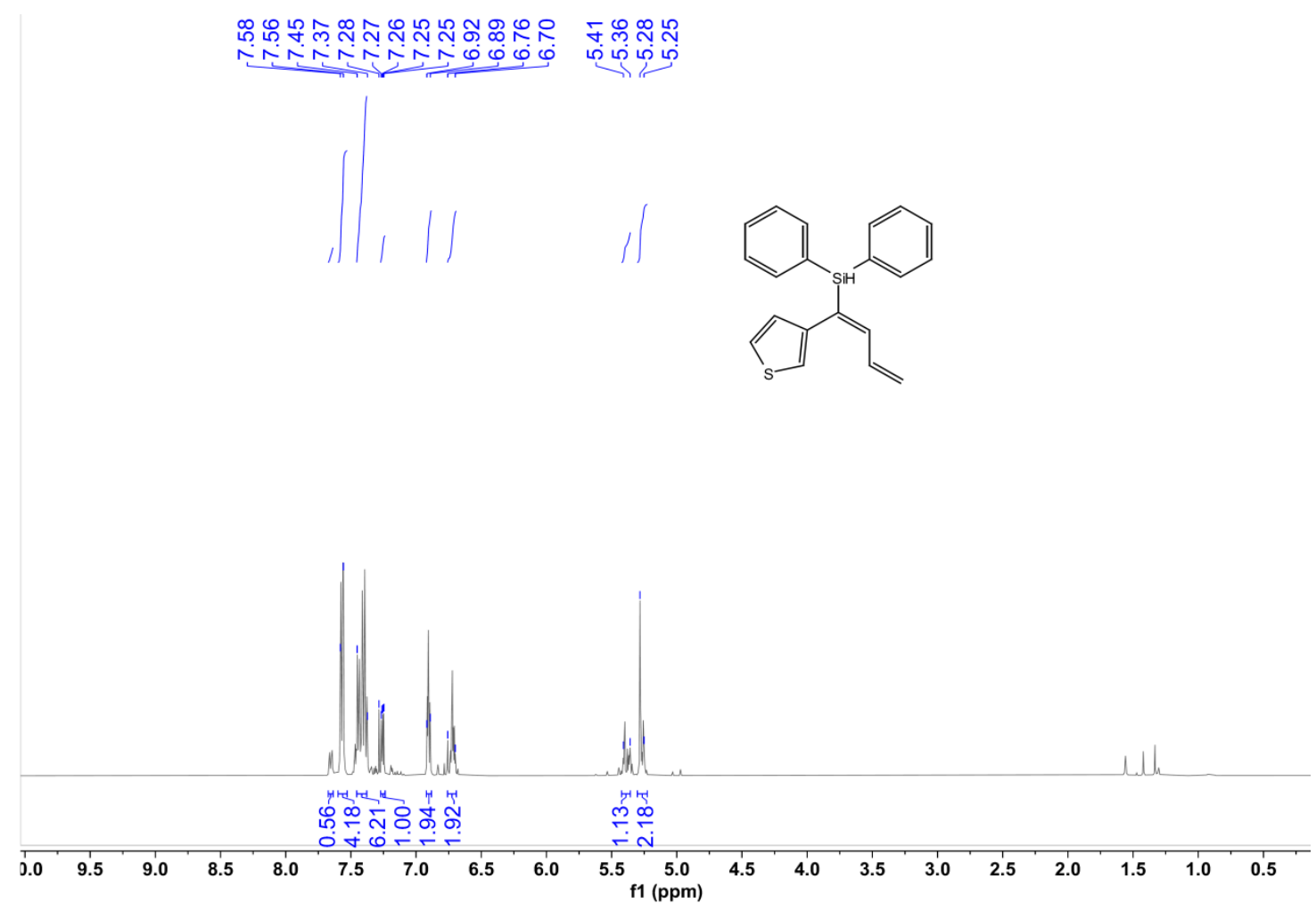

Figure $\mathrm{S67}{ }^{1} \mathrm{H}$ NMR $\left(\mathrm{CDCl}_{3}, 400 \mathrm{M}\right)$ spectrum of $3 \mathrm{q}^{\prime}$ 

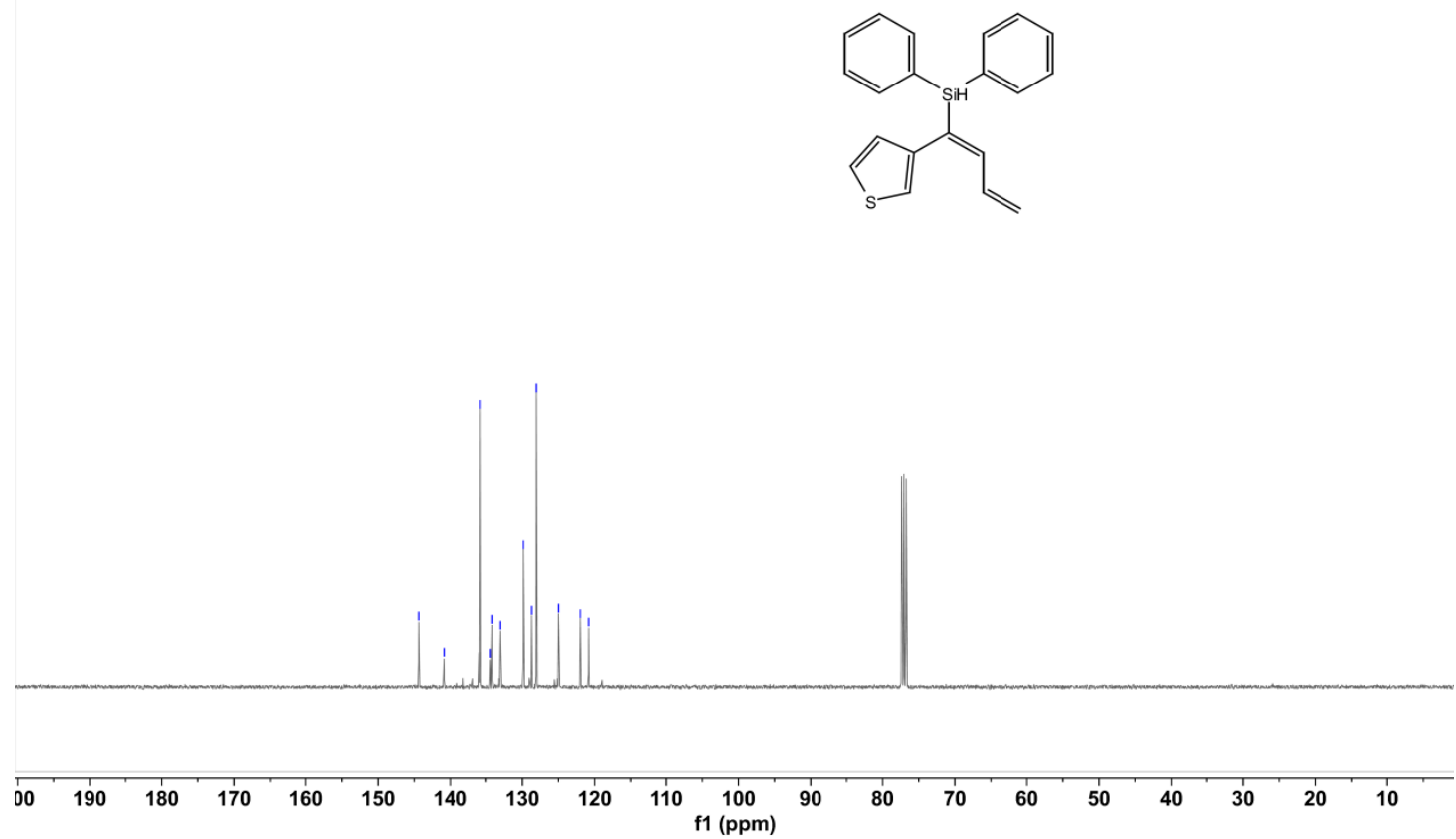

Figure $568{ }^{13} \mathrm{C}$ NMR $\left(\mathrm{CDCl}_{3}, 100 \mathrm{M}\right)$ spectrum of $3 \mathrm{q}^{\prime}$

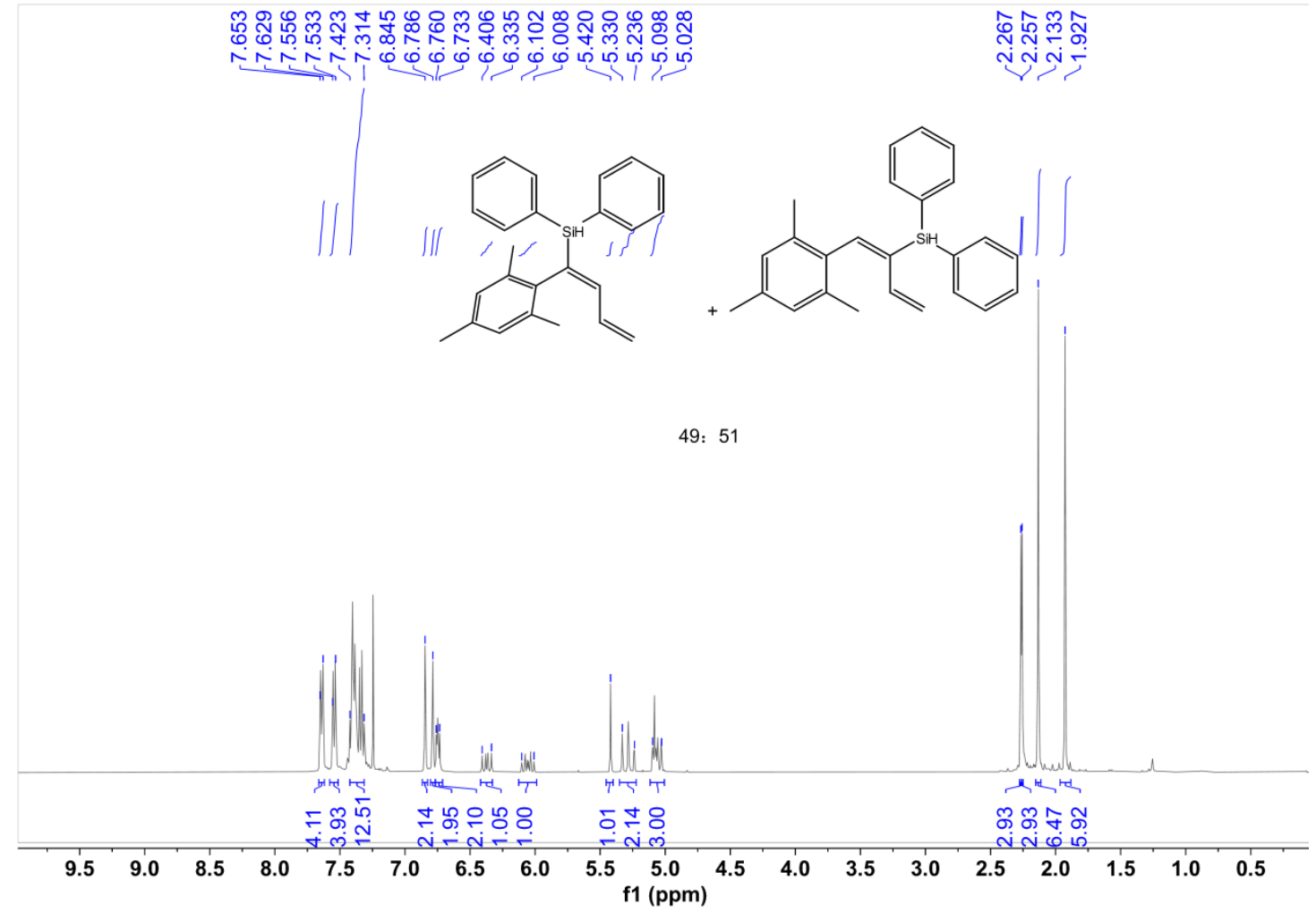

Figure $569{ }^{1} \mathrm{H}$ NMR $\left(\mathrm{CDCl}_{3}, 400 \mathrm{M}\right)$ spectrum of the mixture of $3 \mathrm{~s}^{\prime}$ and $4 \mathrm{~s}^{\prime}$ 


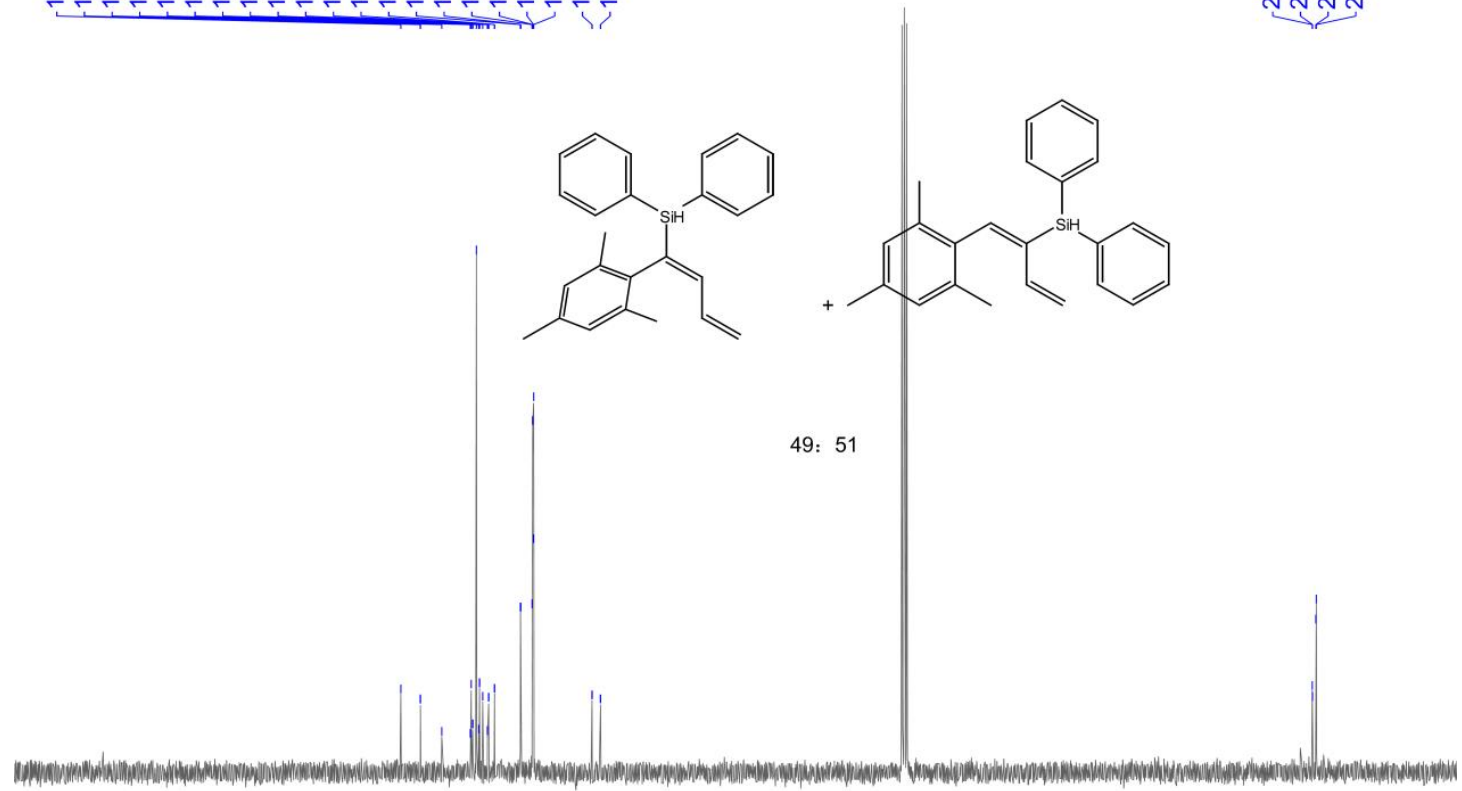

$\begin{array}{llllllllll}190 & 180 & 170 & 160 & 150 & 140 & 130 & 120 & 110 & 100\end{array}$

80

f1 (ppm)

Figure $\mathrm{S70}^{13} \mathrm{C} \mathrm{NMR}\left(\mathrm{CDCl}_{3}, 100 \mathrm{M}\right)$ spectrum of the mixture of $3 \mathrm{~s}^{\prime}$ and $4 \mathrm{~s}^{\prime}$

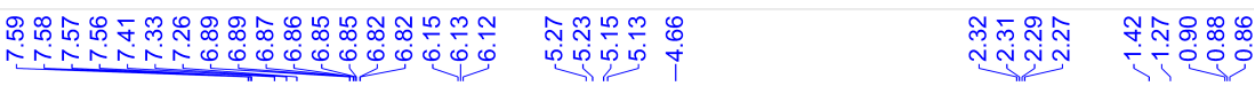

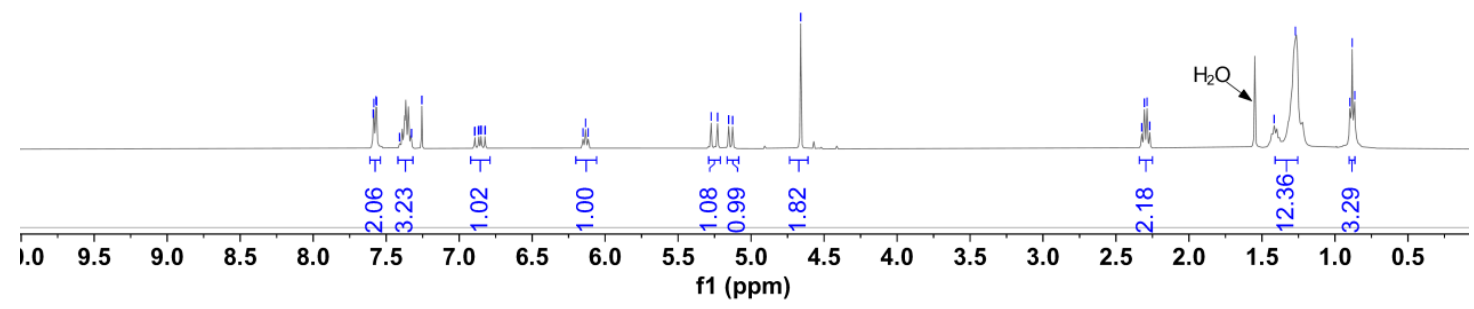

Figure S71 ${ }^{1} \mathrm{H}$ NMR $\left(\mathrm{CDCl}_{3}, 400 \mathrm{M}\right)$ spectrum of $4 \mathrm{t}$ 

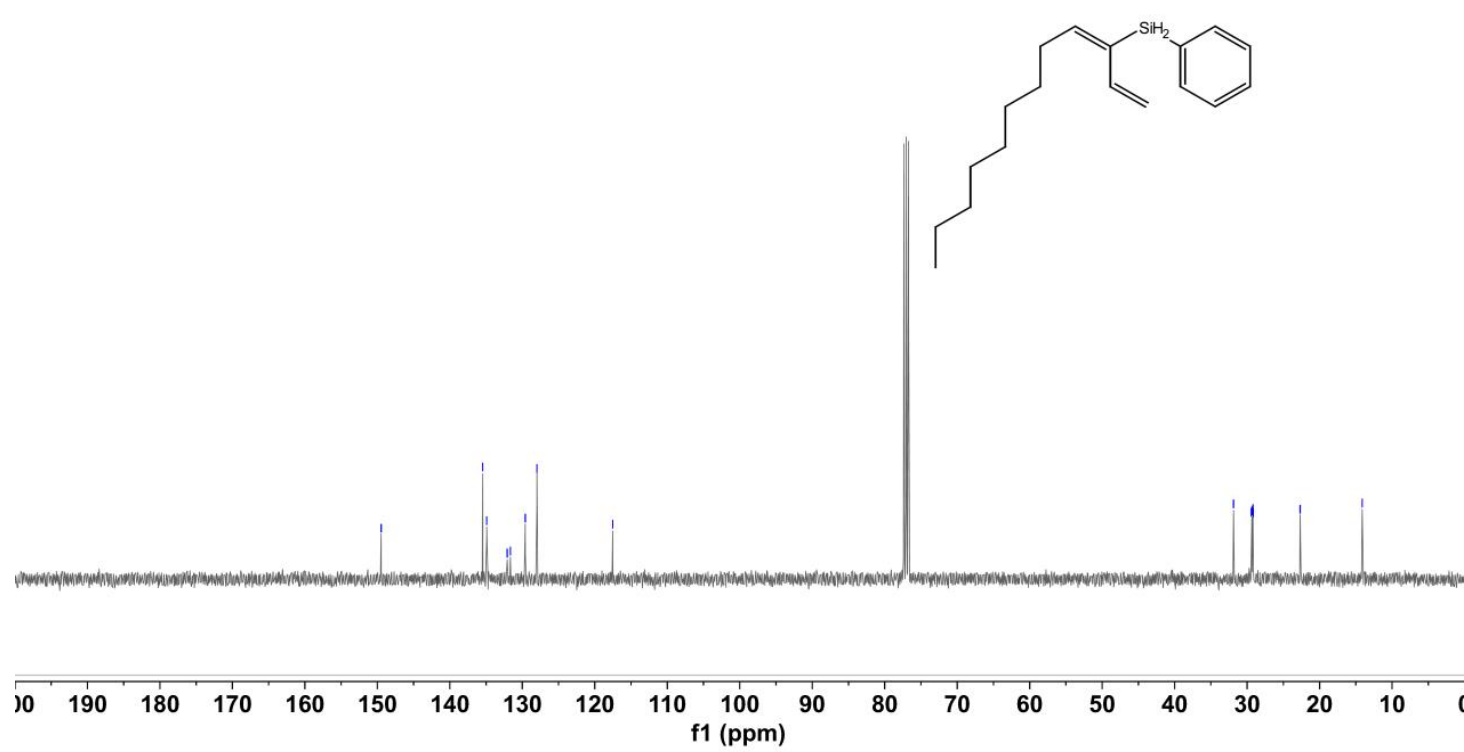

Figure $\mathrm{S}_{2}{ }^{13} \mathrm{C}$ NMR $\left(\mathrm{CDCl}_{3}, 100 \mathrm{M}\right)$ spectrum of $4 \mathrm{t}$

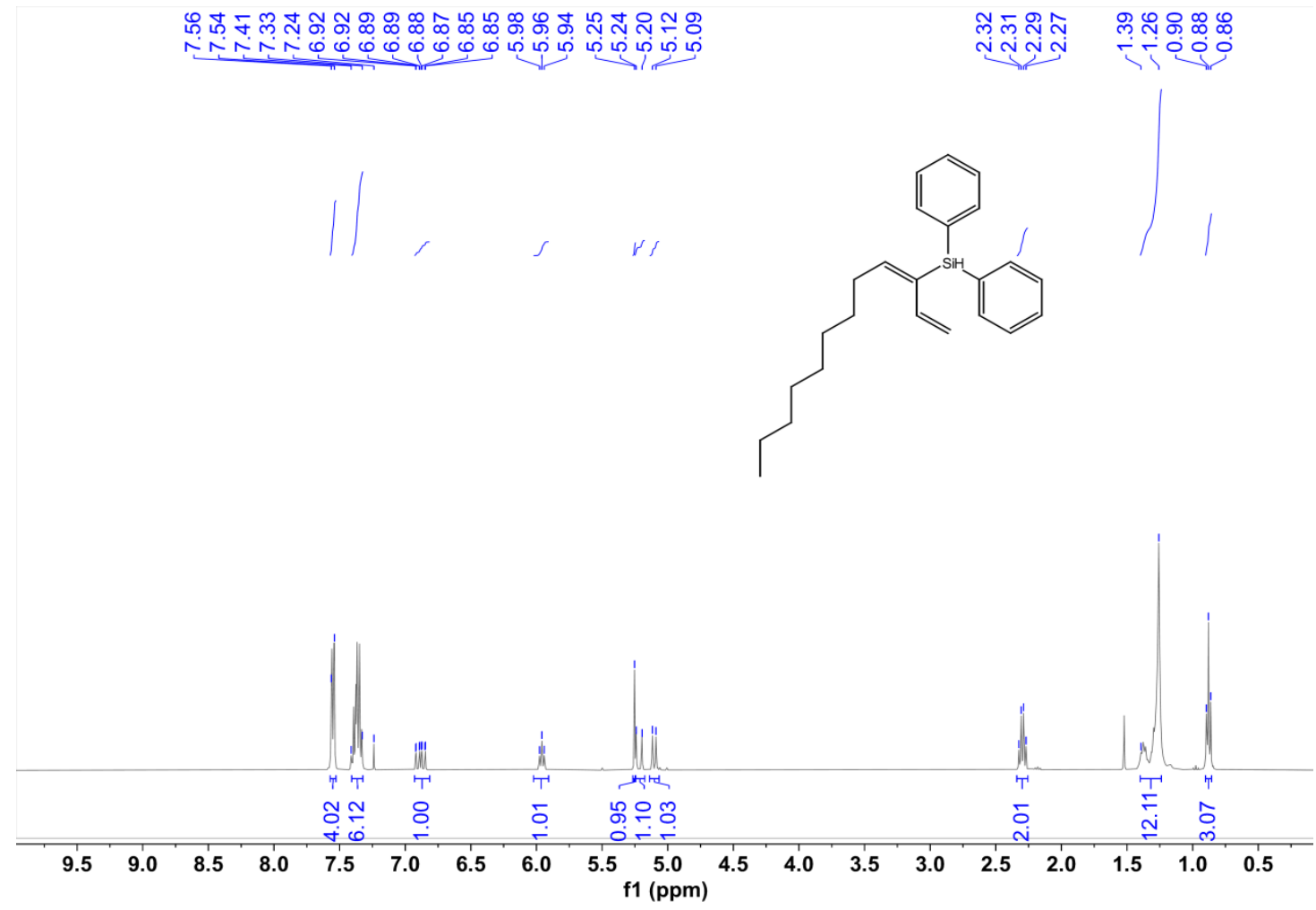

Figure $\mathrm{S73}^{1} \mathrm{H} \mathrm{NMR}\left(\mathrm{CDCl}_{3}, 400 \mathrm{M}\right)$ spectrum of $4 \mathrm{t}^{\prime}$ 


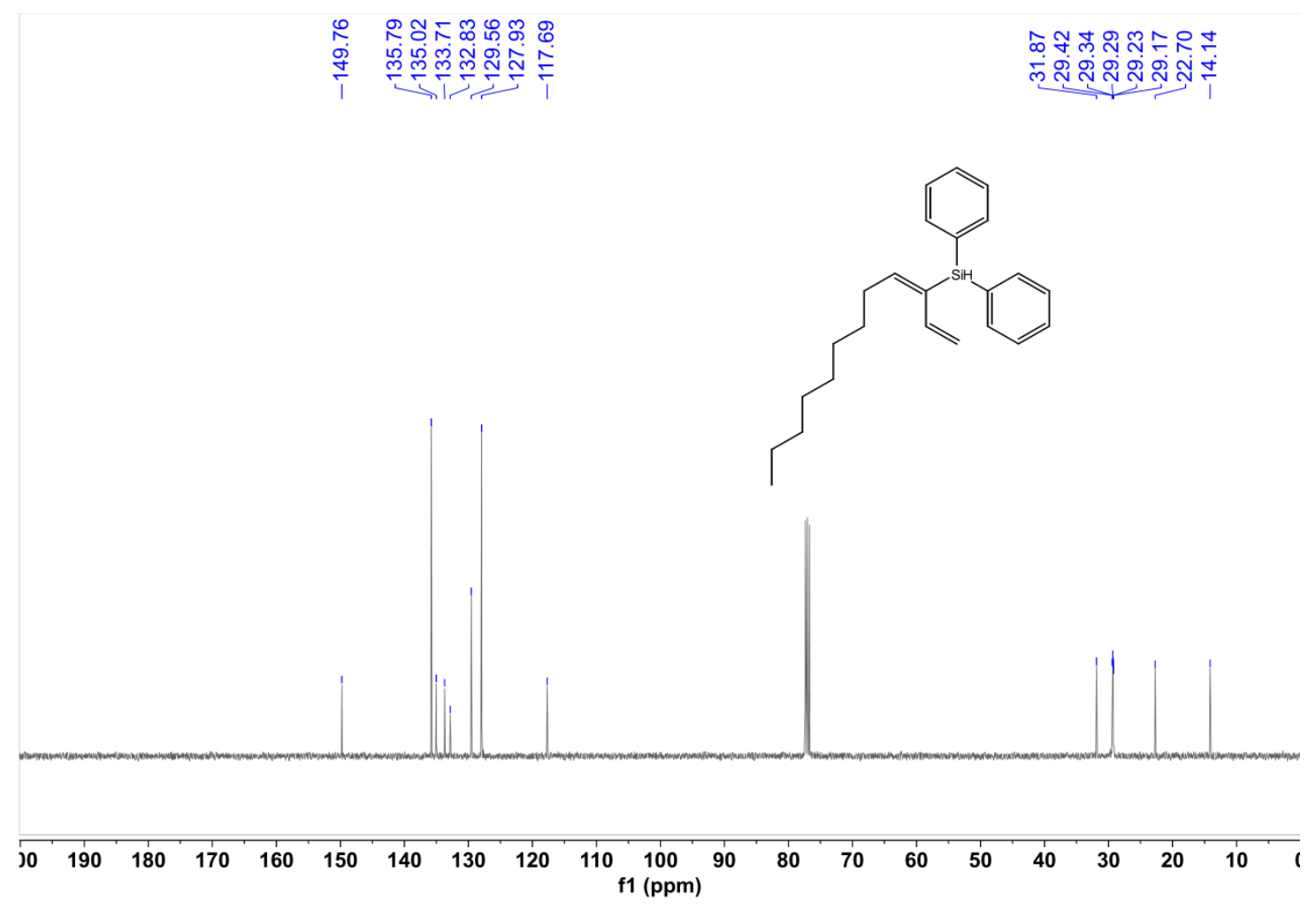

Figure S74 ${ }^{13} \mathrm{C}$ NMR $\left(\mathrm{CDCl}_{3}, 100 \mathrm{M}\right)$ spectrum of $4 \mathrm{t}^{\prime}$

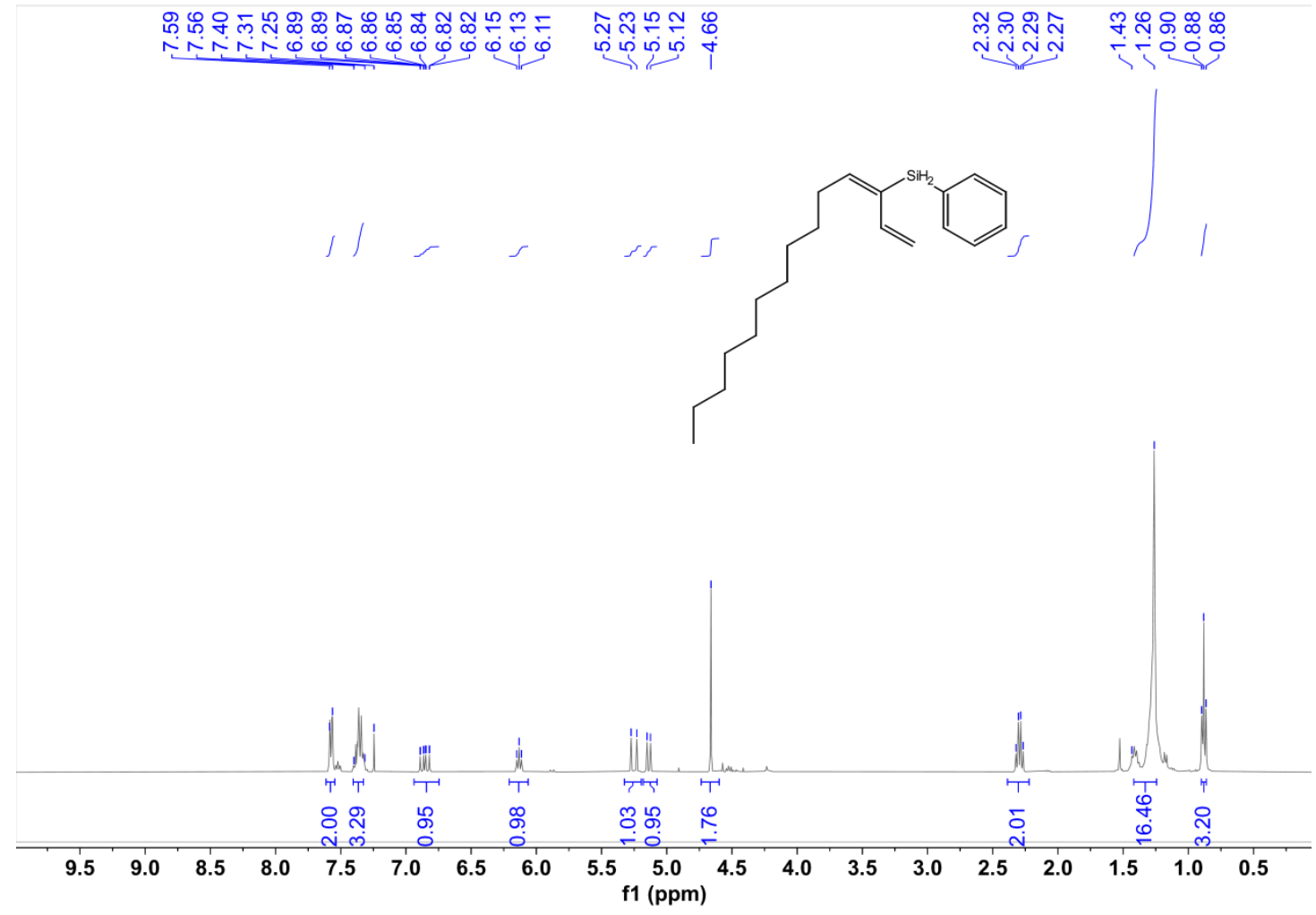

Figure $\mathrm{S75}{ }^{1} \mathrm{H} \mathrm{NMR}\left(\mathrm{CDCl}_{3}, 400 \mathrm{M}\right)$ spectrum of $4 \mathrm{u}$ 


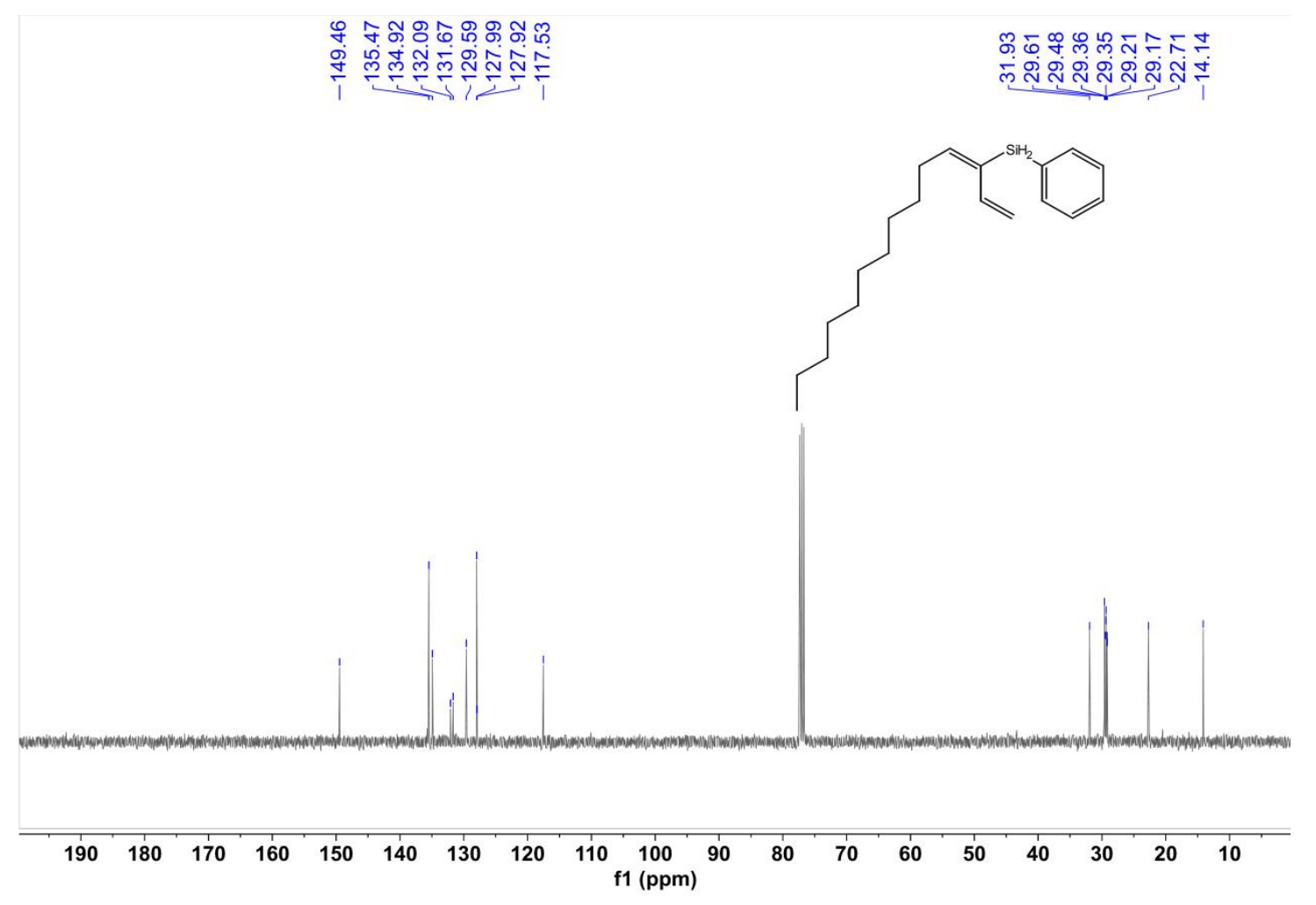

Figure $\mathrm{S76}^{13} \mathrm{C} \mathrm{NMR}\left(\mathrm{CDCl}_{3}, 100 \mathrm{M}\right)$ spectrum of $4 \mathrm{u}$

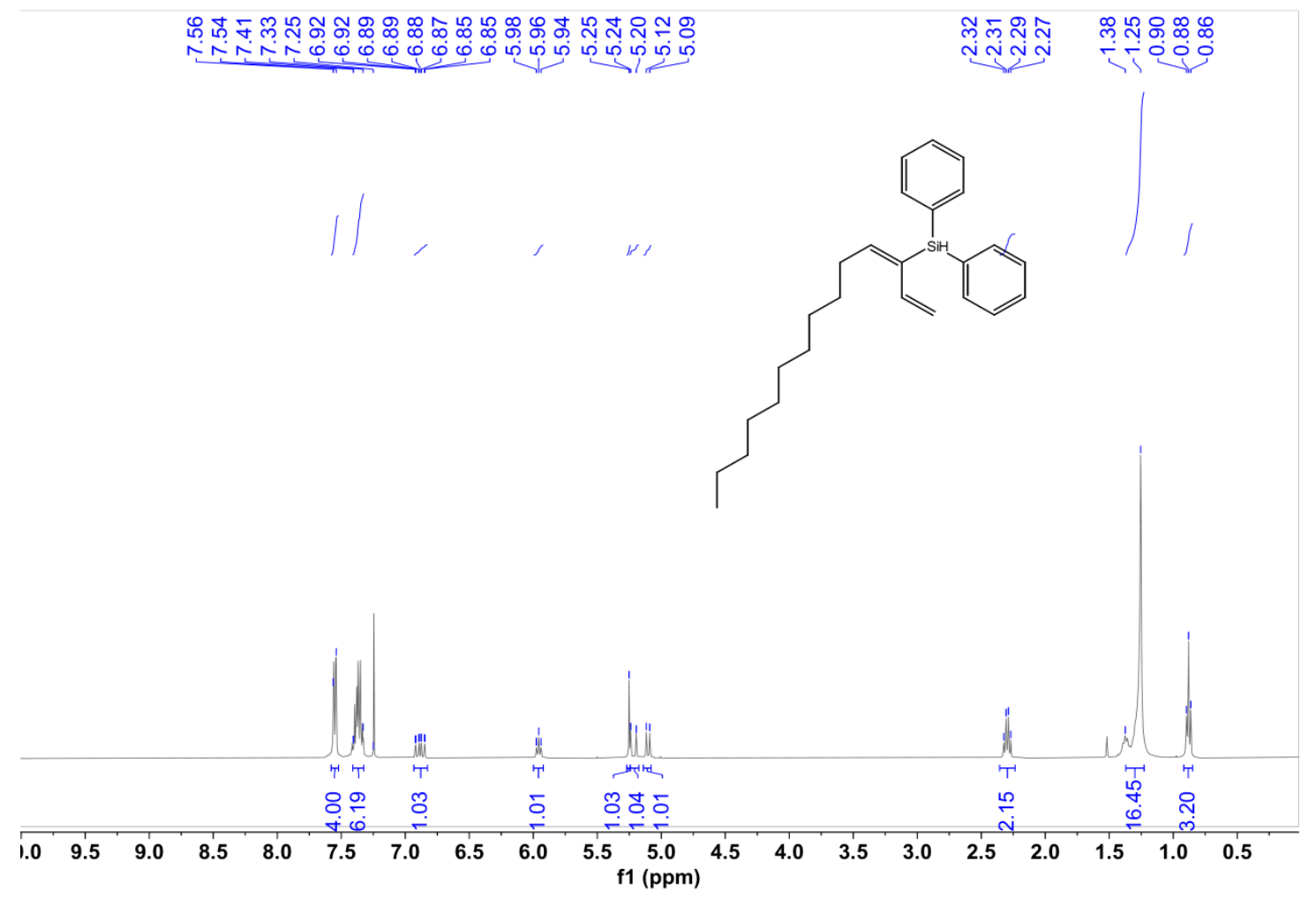

Figure S77 ${ }^{1} \mathrm{H}$ NMR $\left(\mathrm{CDCl}_{3}, 400 \mathrm{M}\right)$ spectrum of $4 \mathrm{u}^{\prime}$ 


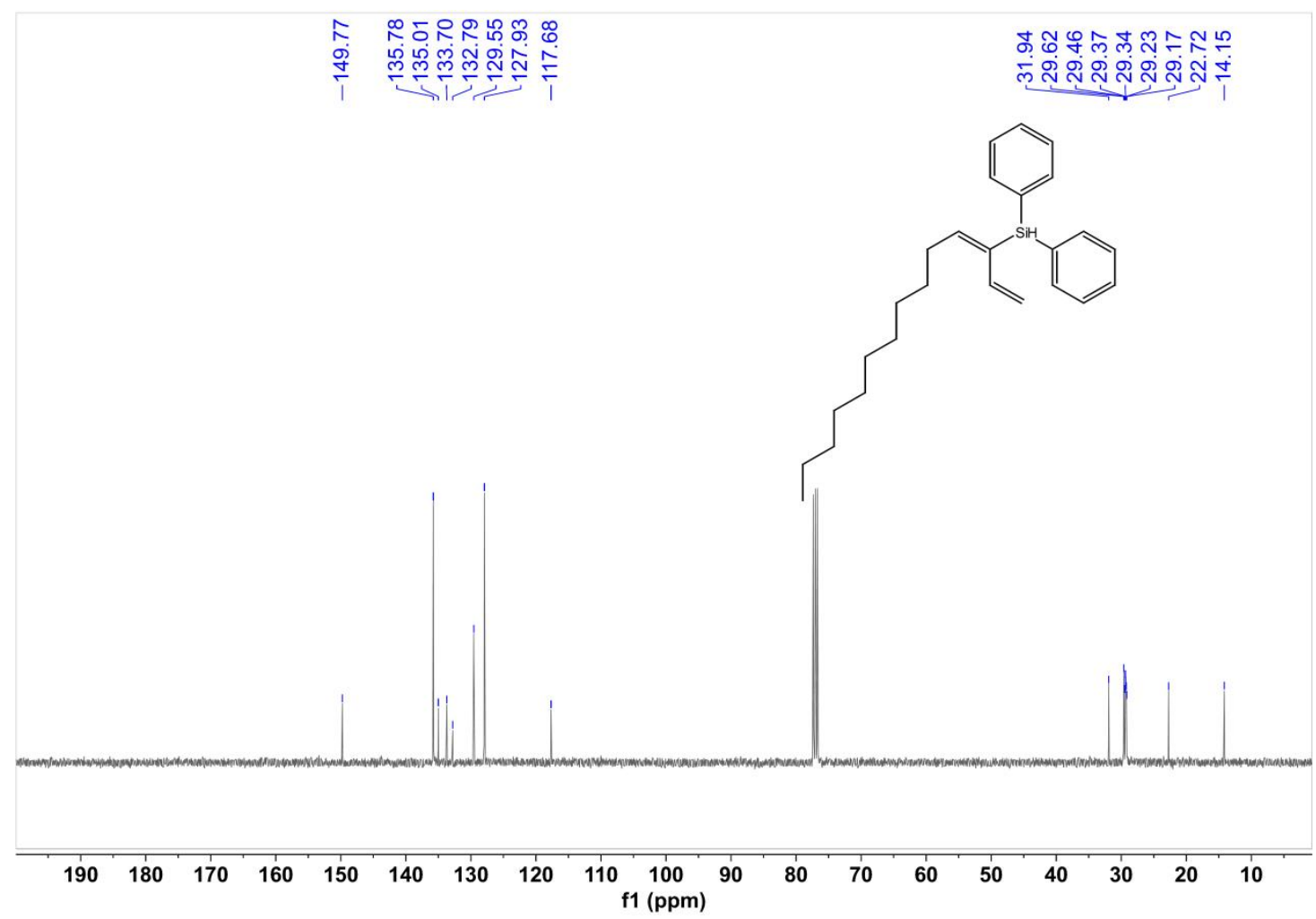

Figure $\mathrm{S78}^{13} \mathrm{C} \mathrm{NMR}\left(\mathrm{CDCl}_{3}, 100 \mathrm{M}\right)$ spectrum of $4 \mathrm{u}^{\prime}$
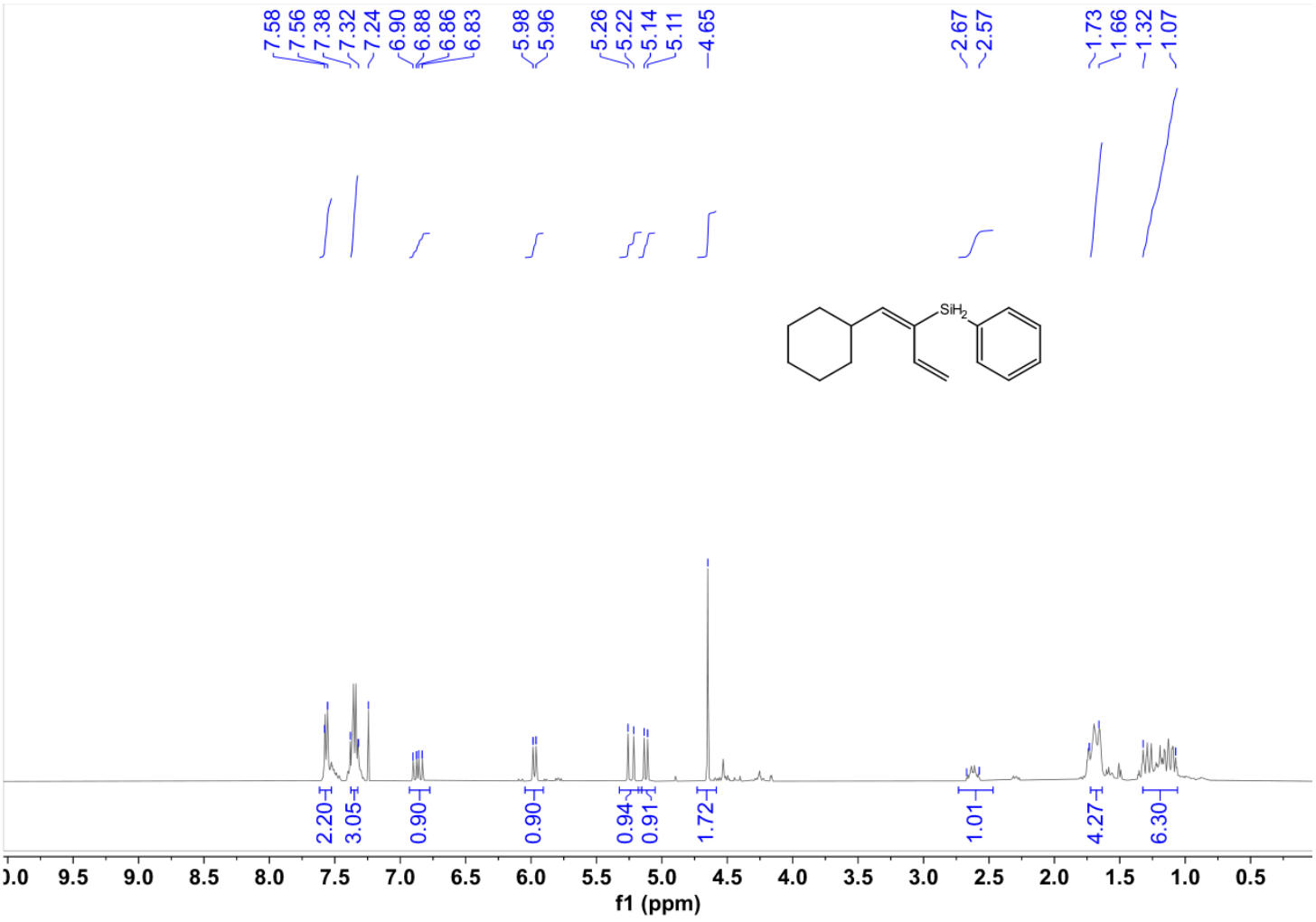

Figure $\mathrm{S79}{ }^{1} \mathrm{H} \mathrm{NMR}\left(\mathrm{CDCl}_{3}, 400 \mathrm{M}\right)$ spectrum of $4 \mathrm{v}$ 

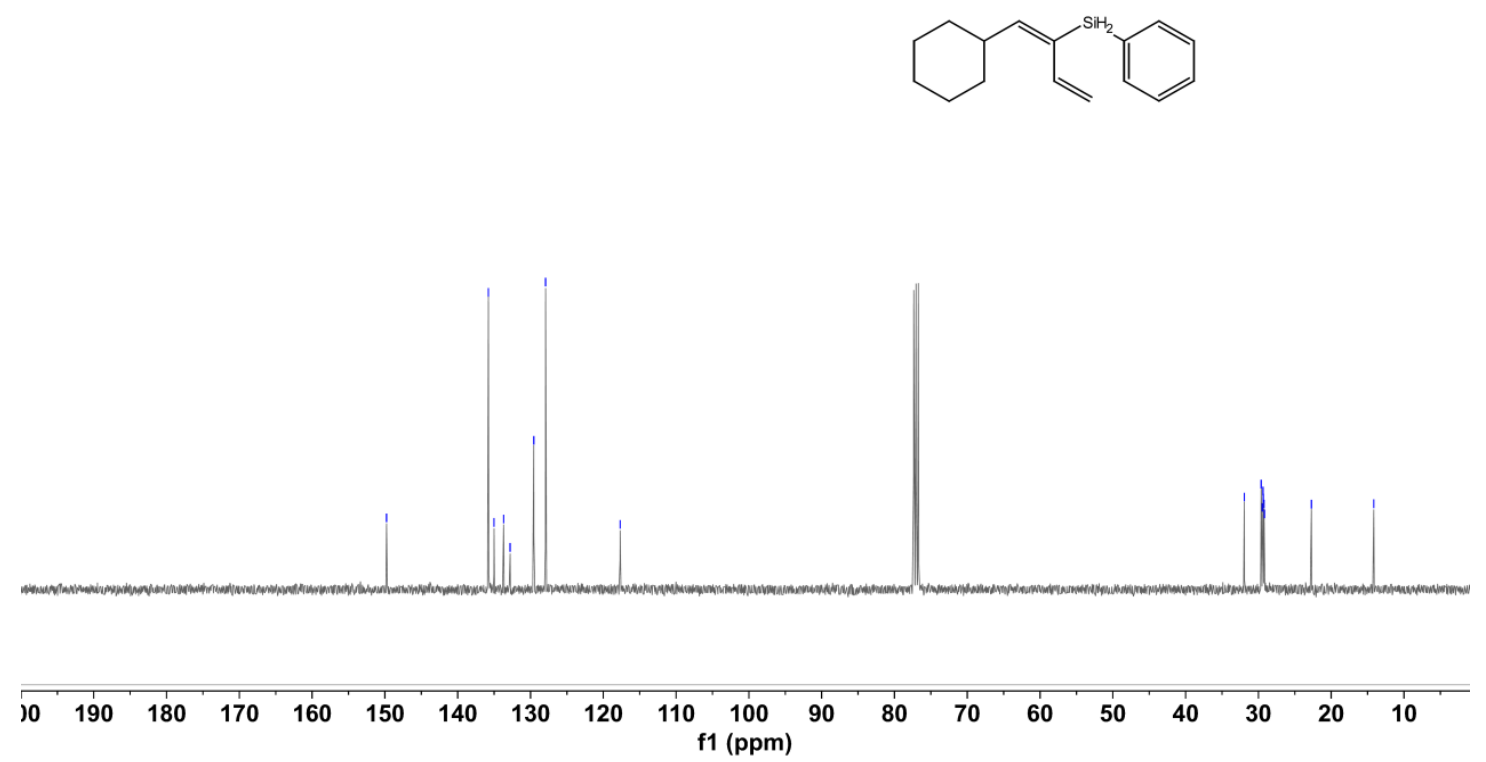

Figure $\mathrm{S80}^{13} \mathrm{C} \mathrm{NMR}\left(\mathrm{CDCl}_{3}, 100 \mathrm{M}\right)$ spectrum of $4 \mathrm{v}$
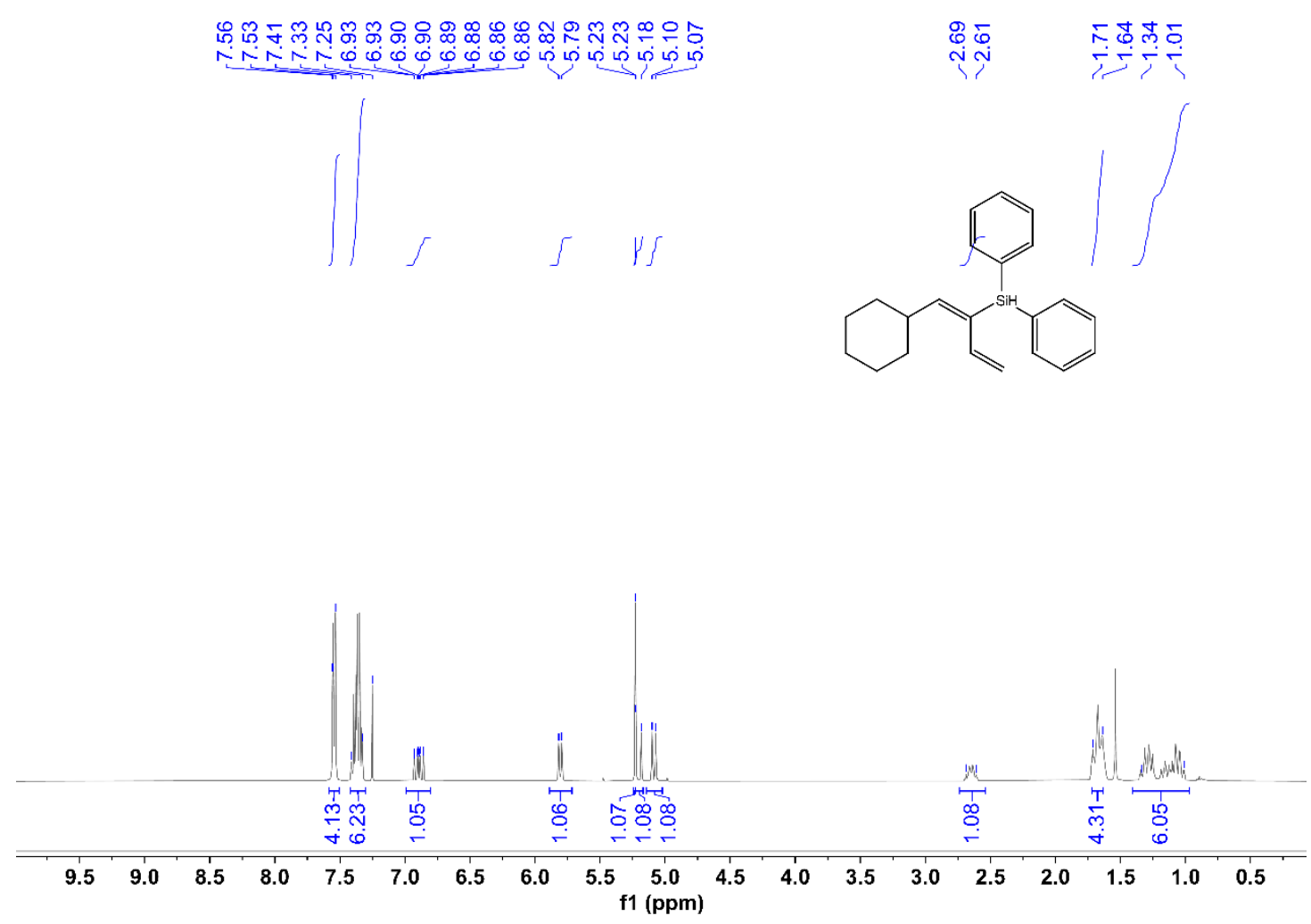

Figure S81 ${ }^{1} \mathrm{H}$ NMR $\left(\mathrm{CDCl}_{3}, 400 \mathrm{M}\right)$ spectrum of $4 \mathrm{v}^{\prime}$ 


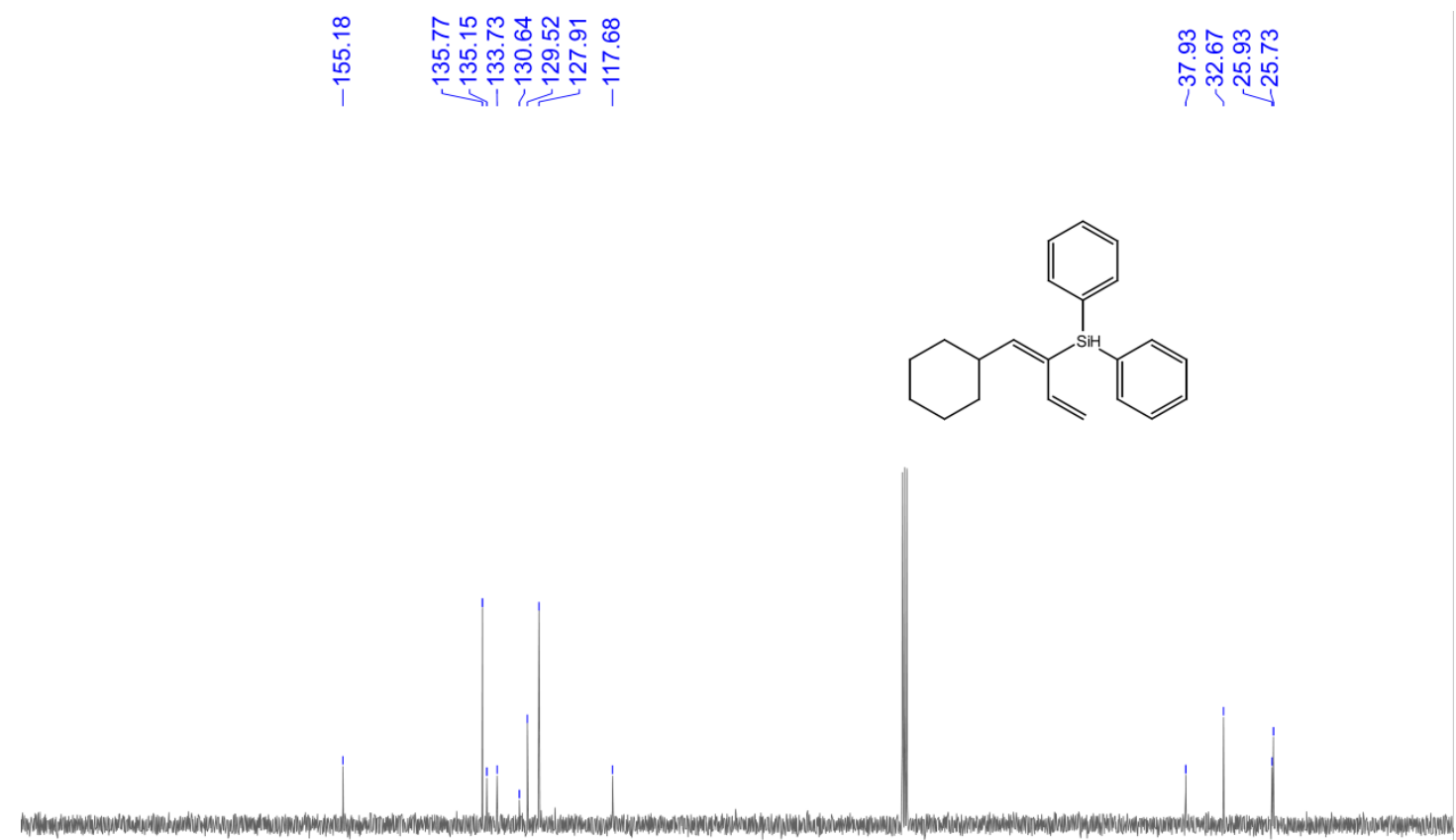

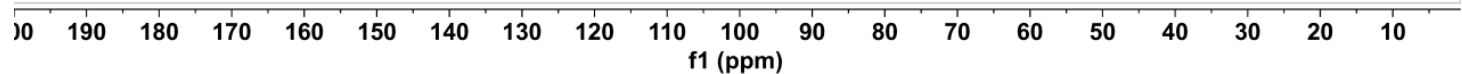

Figure $\mathrm{S82}{ }^{13} \mathrm{C} \mathrm{NMR}\left(\mathrm{CDCl}_{3}, 100 \mathrm{M}\right)$ spectrum of $4 \mathrm{v}^{\prime}$
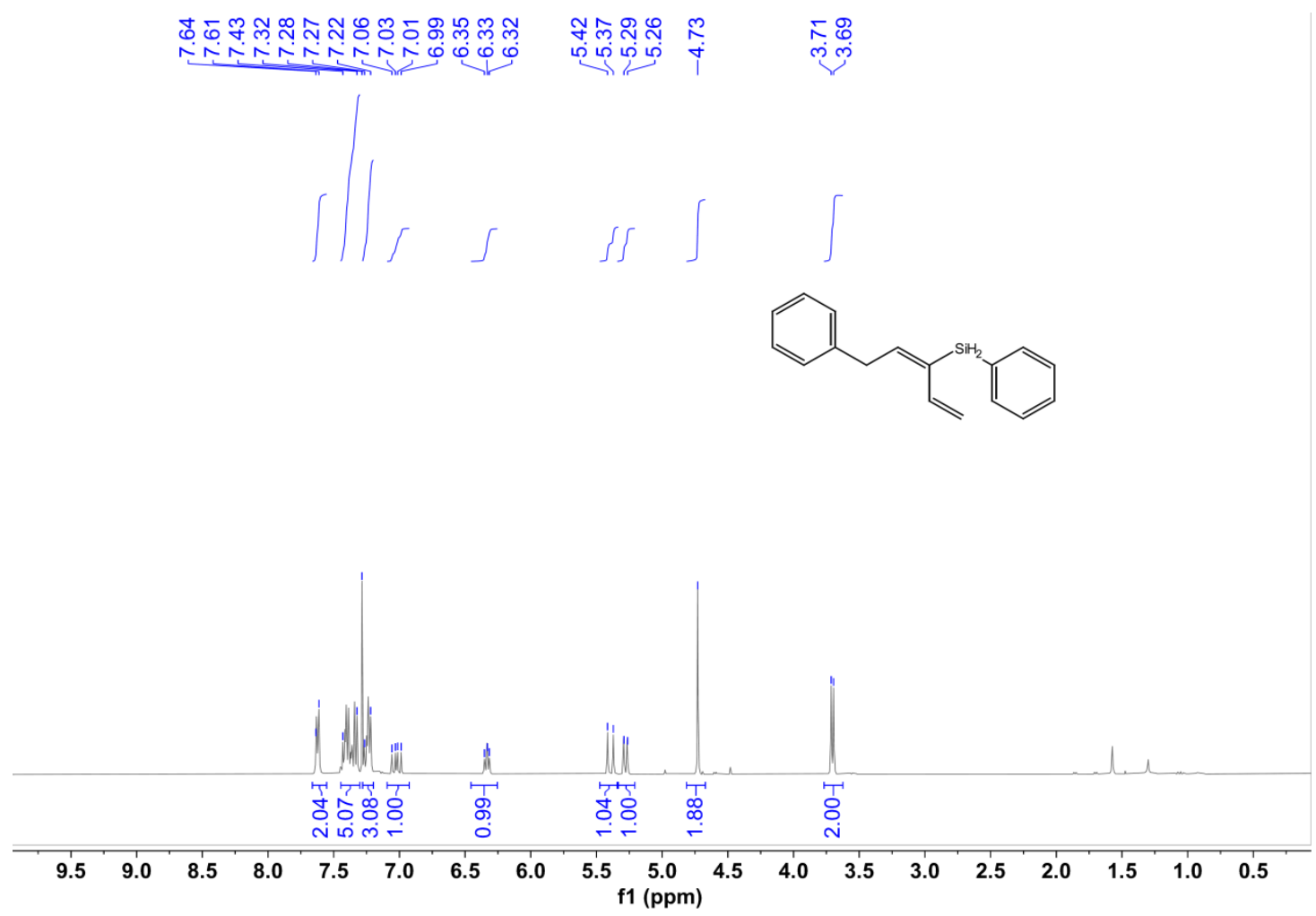

Figure $\mathrm{S83}^{1} \mathrm{H}$ NMR $\left(\mathrm{CDCl}_{3}, 400 \mathrm{M}\right)$ spectrum of $4 \mathrm{w}$ 

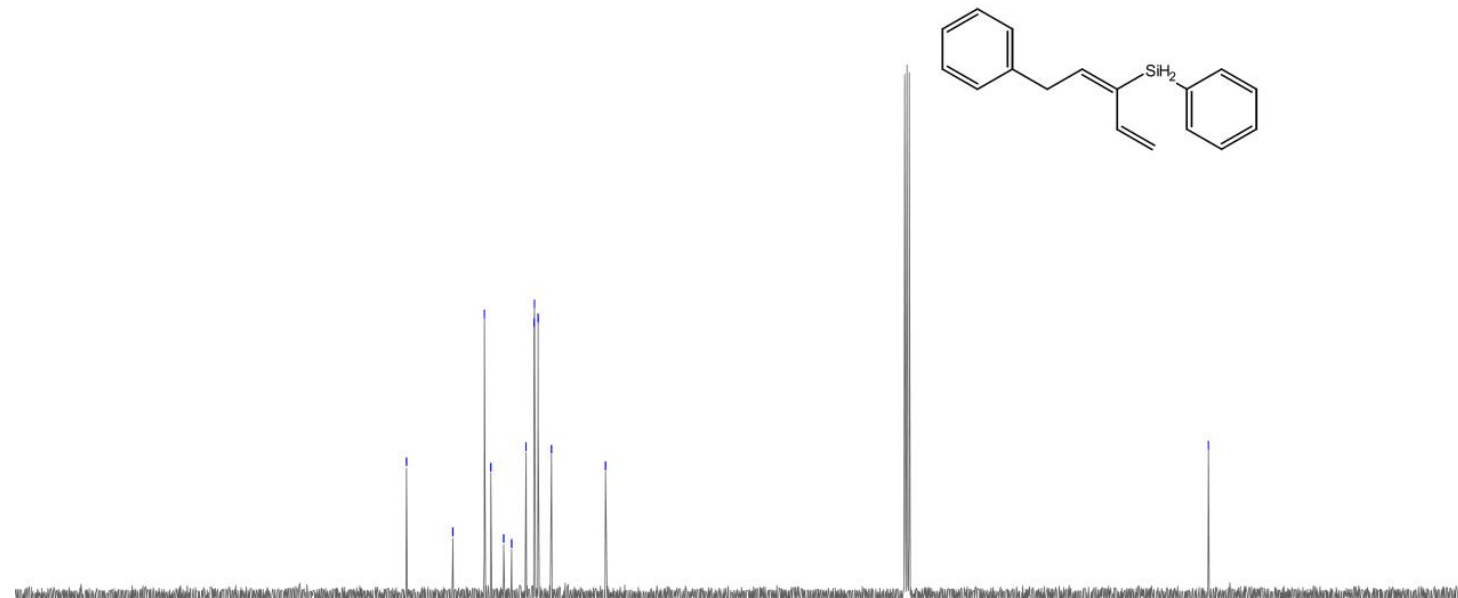

\begin{tabular}{lllllllllllllllllllll}
\hline 00 & 190 & 180 & 170 & 160 & 150 & 140 & 130 & 120 & $\begin{array}{c}110 \\
\mathrm{f} 1(\mathrm{ppm})\end{array}$ & 90 & 80 & 70 & 60 & 50 & 40 & 30 & 20 & 10
\end{tabular}

Figure S84 ${ }^{13} \mathrm{C}$ NMR $\left(\mathrm{CDCl}_{3}, 100 \mathrm{M}\right)$ spectrum of $4 \mathrm{w}$

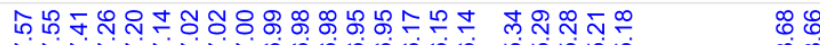

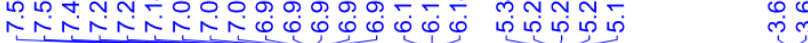
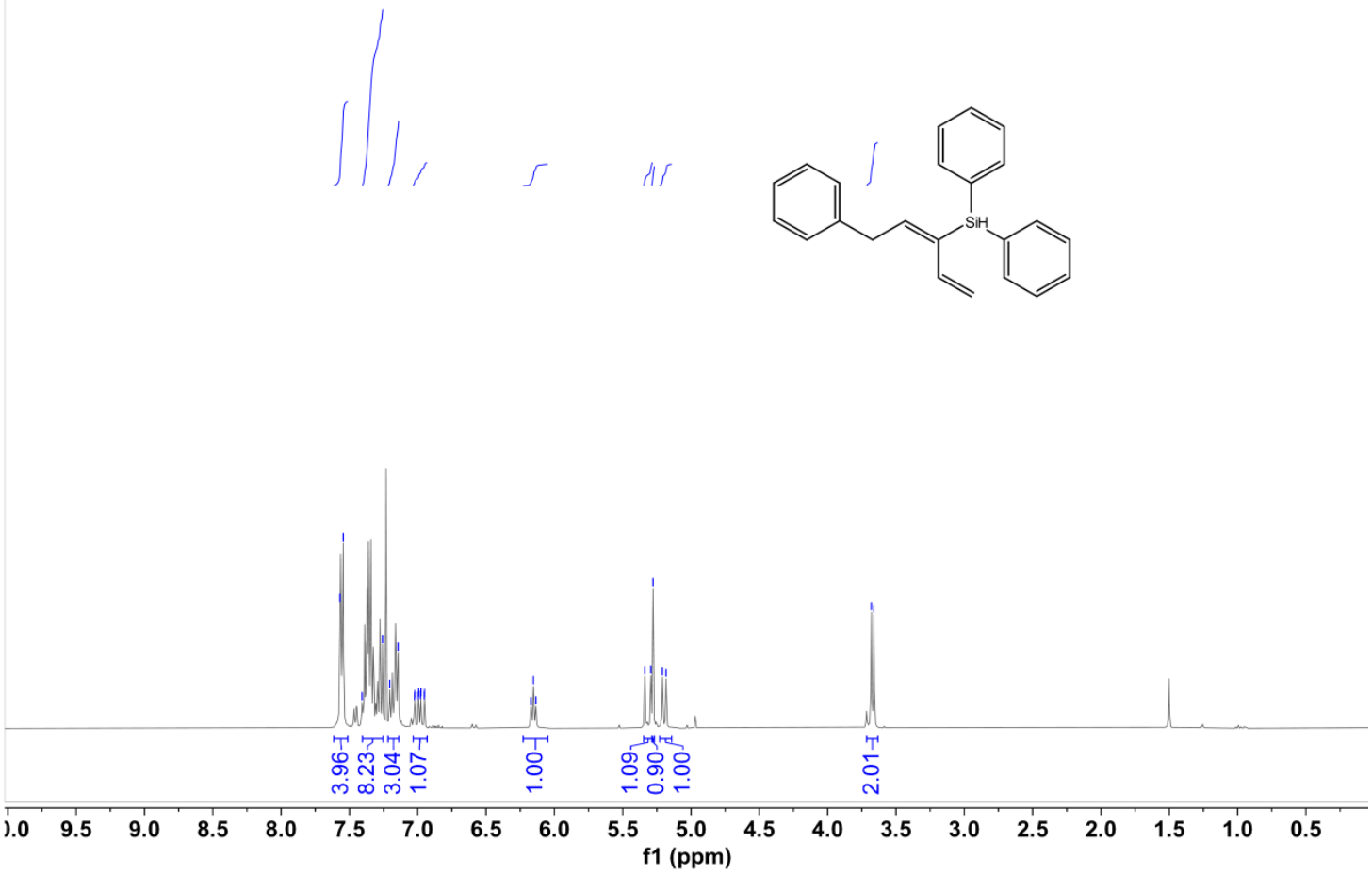

Figure $\mathrm{S85}{ }^{1} \mathrm{H} \mathrm{NMR}\left(\mathrm{CDCl}_{3}, 400 \mathrm{M}\right)$ spectrum of $4 \mathrm{w}^{\prime}$ 

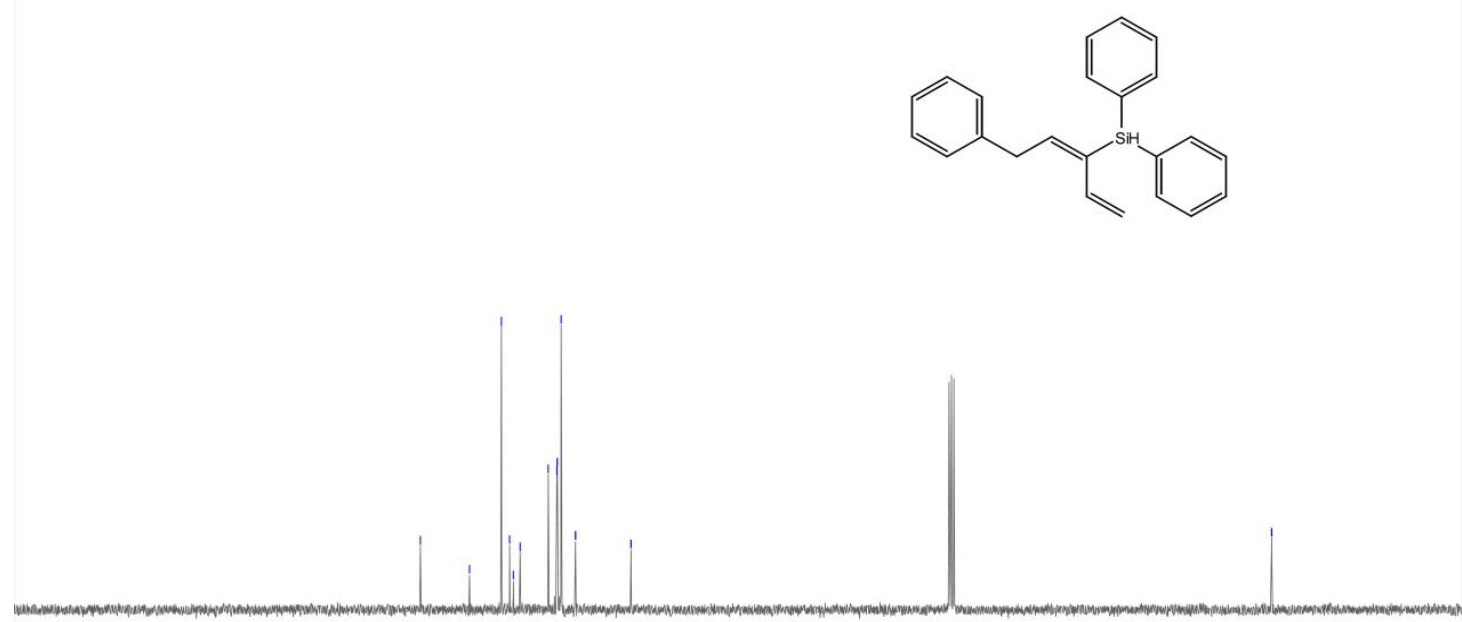

$\begin{array}{llllllllll}190 & 180 & 170 & 160 & 150 & 140 & 130 & 120 & 110 & 100\end{array}$

Figure $\mathrm{S86}^{13} \mathrm{C} \mathrm{NMR}\left(\mathrm{CDCl}_{3}, 100 \mathrm{M}\right)$ spectrum of $4 \mathrm{w}^{\prime}$
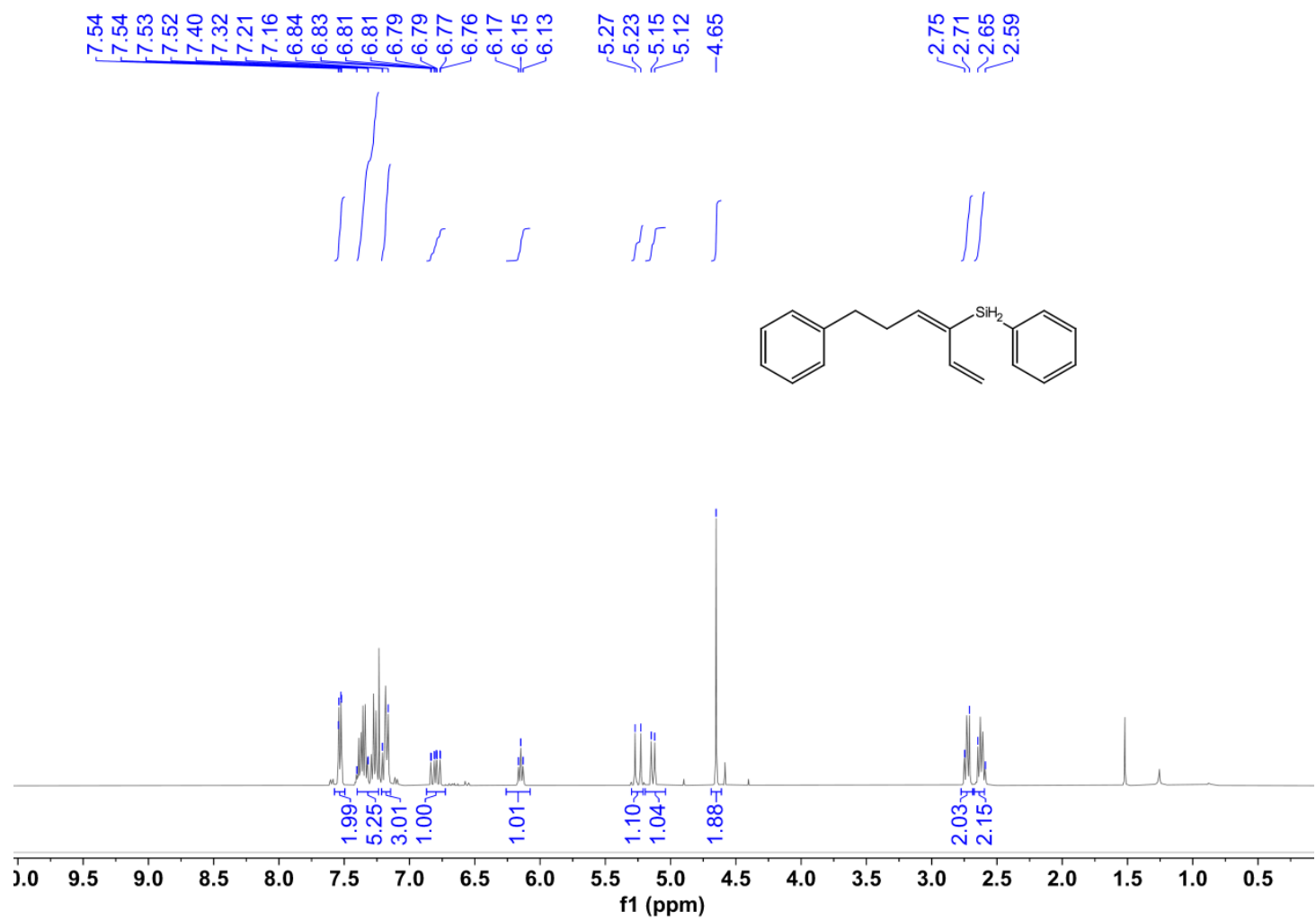

Figure $\mathrm{S87}{ }^{1} \mathrm{H}$ NMR $\left(\mathrm{CDCl}_{3}, 400 \mathrm{M}\right)$ spectrum of $4 \mathrm{x}$ 

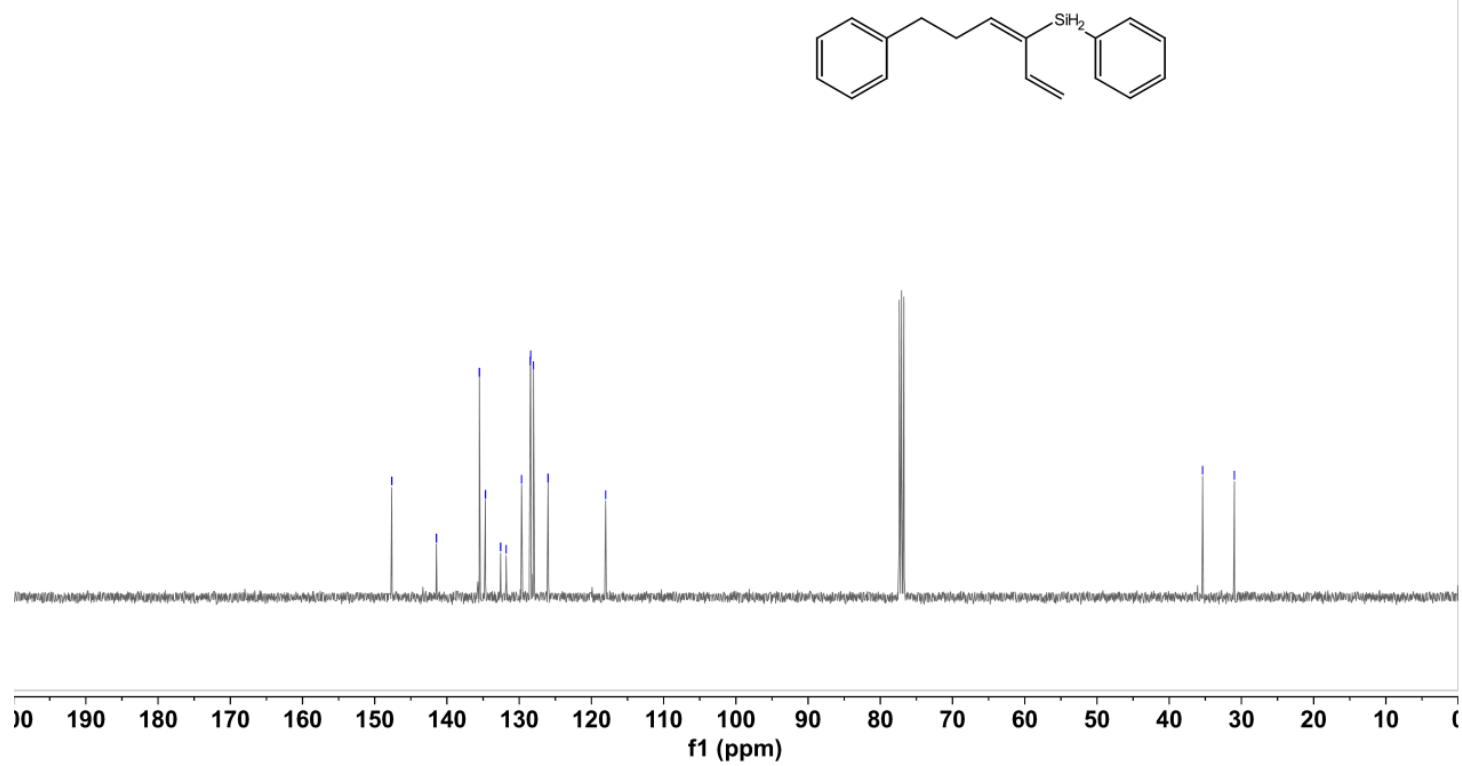

Figure $\mathrm{Ss8}^{13} \mathrm{C} \mathrm{NMR}\left(\mathrm{CDCl}_{3}, 100 \mathrm{M}\right)$ spectrum of $4 \mathrm{x}$

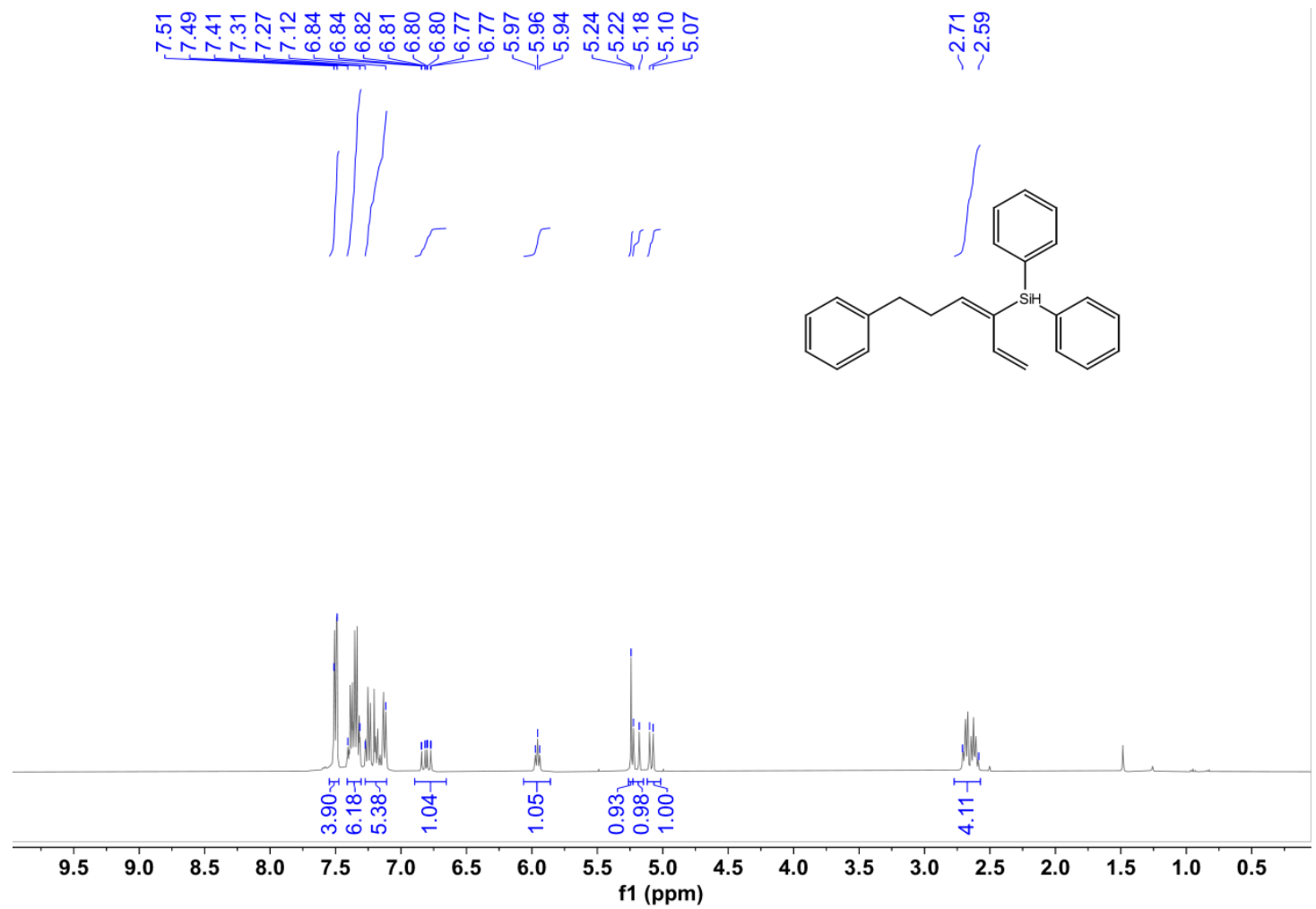

Figure $\mathrm{S89}^{1} \mathrm{H}$ NMR $\left(\mathrm{CDCl}_{3}, 400 \mathrm{M}\right)$ spectrum of $4 \mathrm{x}^{\prime}$ 

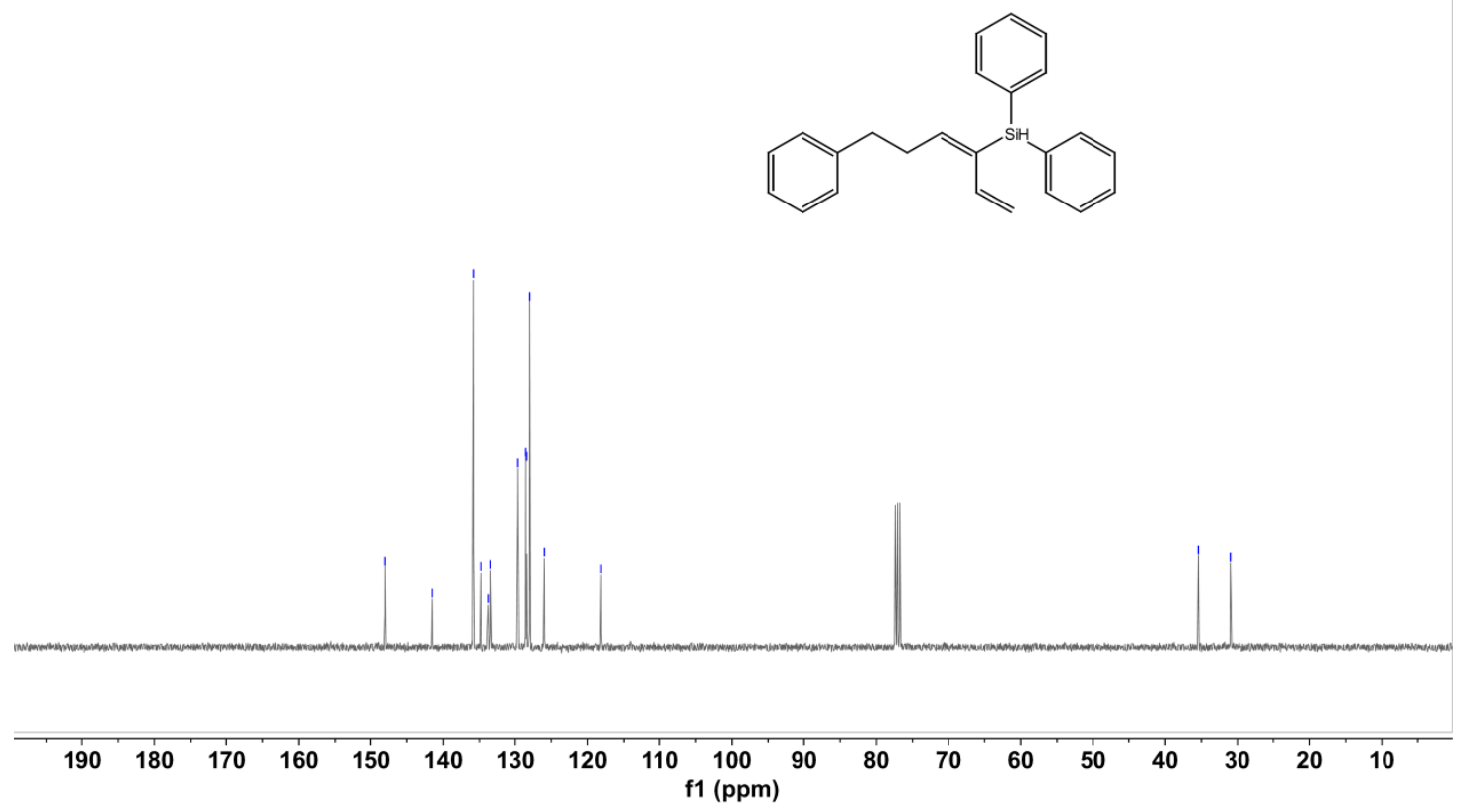

Figure S90 ${ }^{13} \mathrm{C} \mathrm{NMR}\left(\mathrm{CDCl}_{3}, 100 \mathrm{M}\right)$ spectrum of $4 \mathrm{x}^{\prime}$

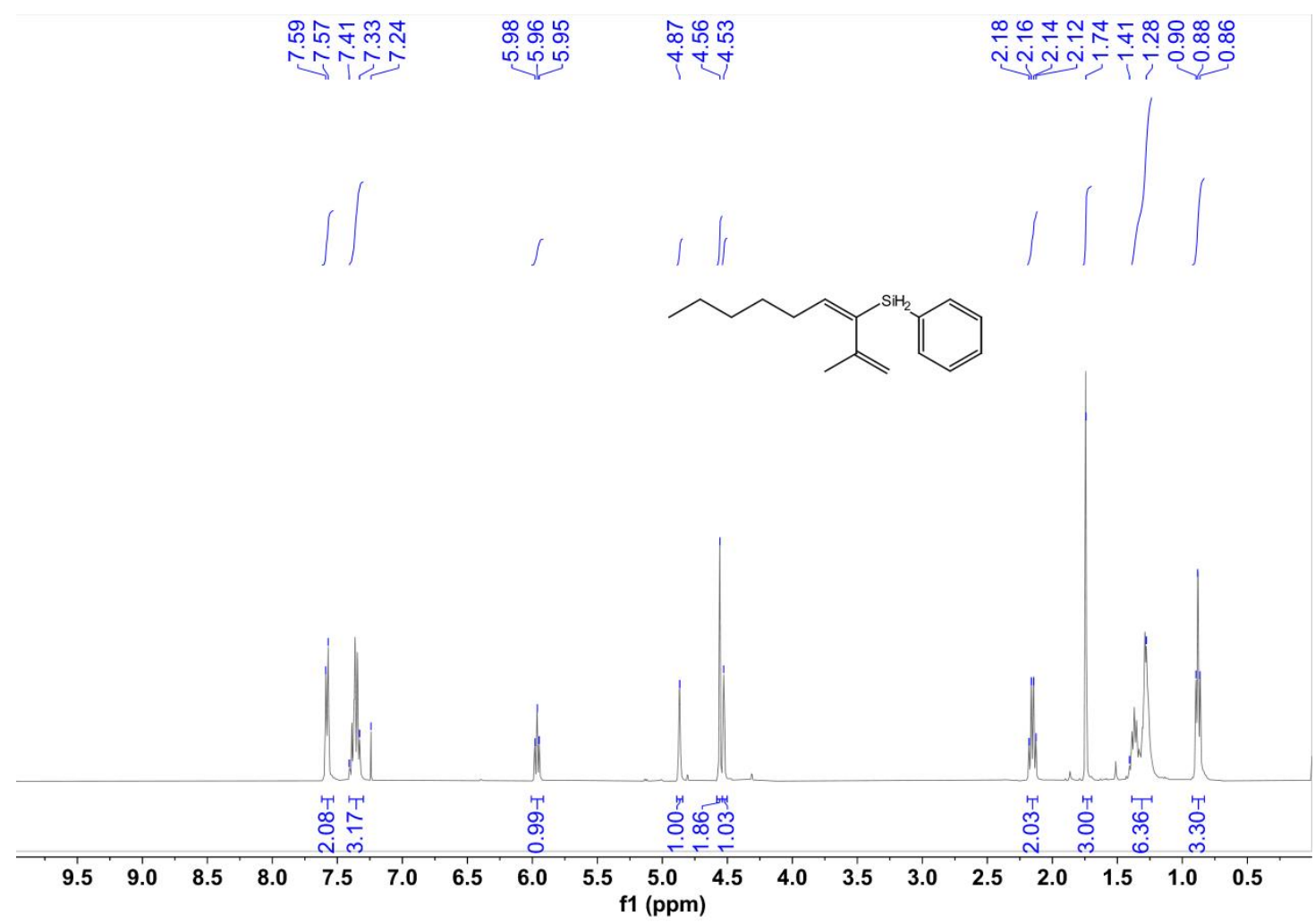

Figure $\mathrm{S91}{ }^{1} \mathrm{H}$ NMR $\left(\mathrm{CDCl}_{3}, 400 \mathrm{M}\right)$ spectrum of $4 \mathrm{y}$ 


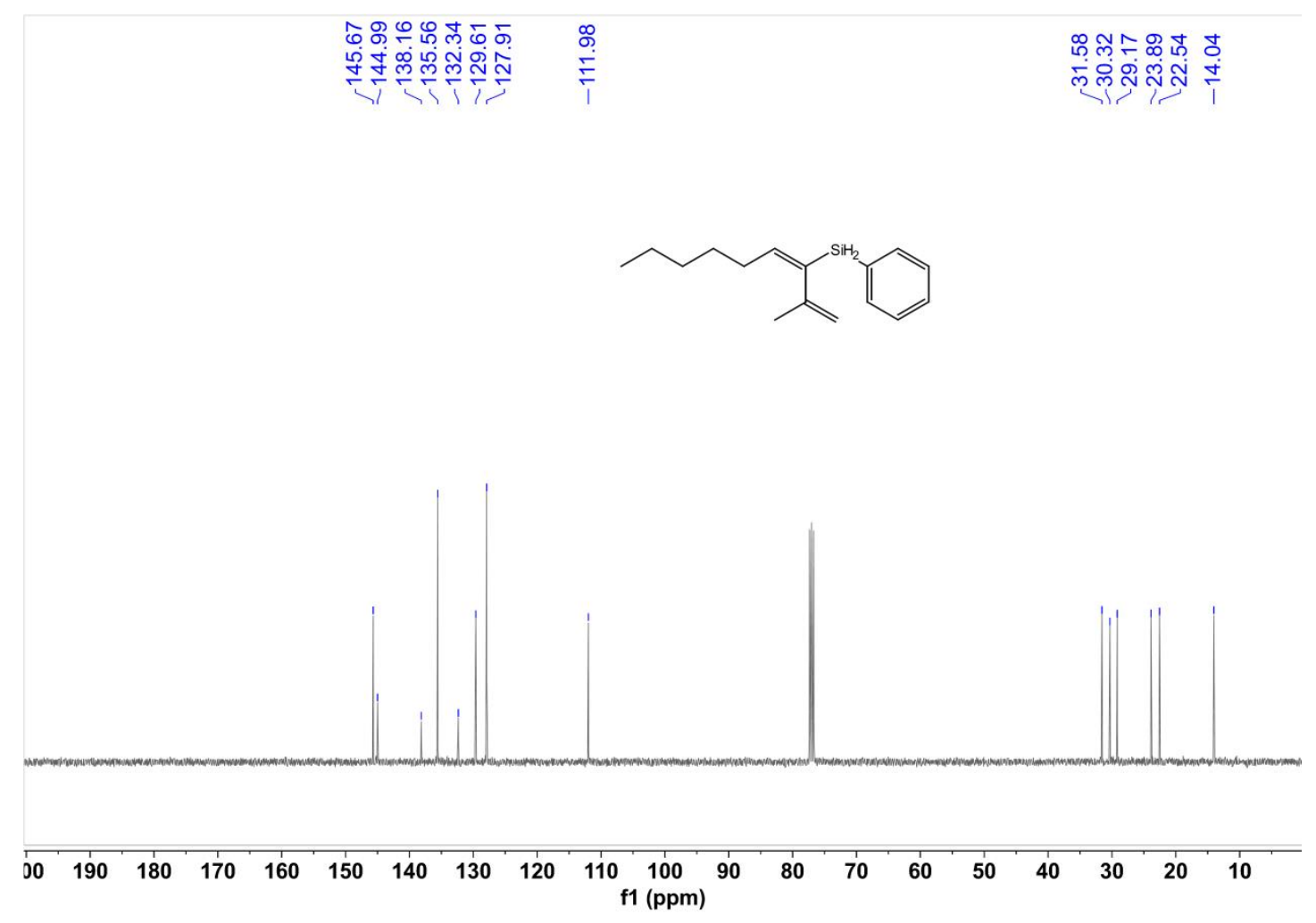

Figure $\mathrm{S92}{ }^{13} \mathrm{C} \mathrm{NMR}\left(\mathrm{CDCl}_{3}, 100 \mathrm{M}\right)$ spectrum of $4 \mathrm{y}$

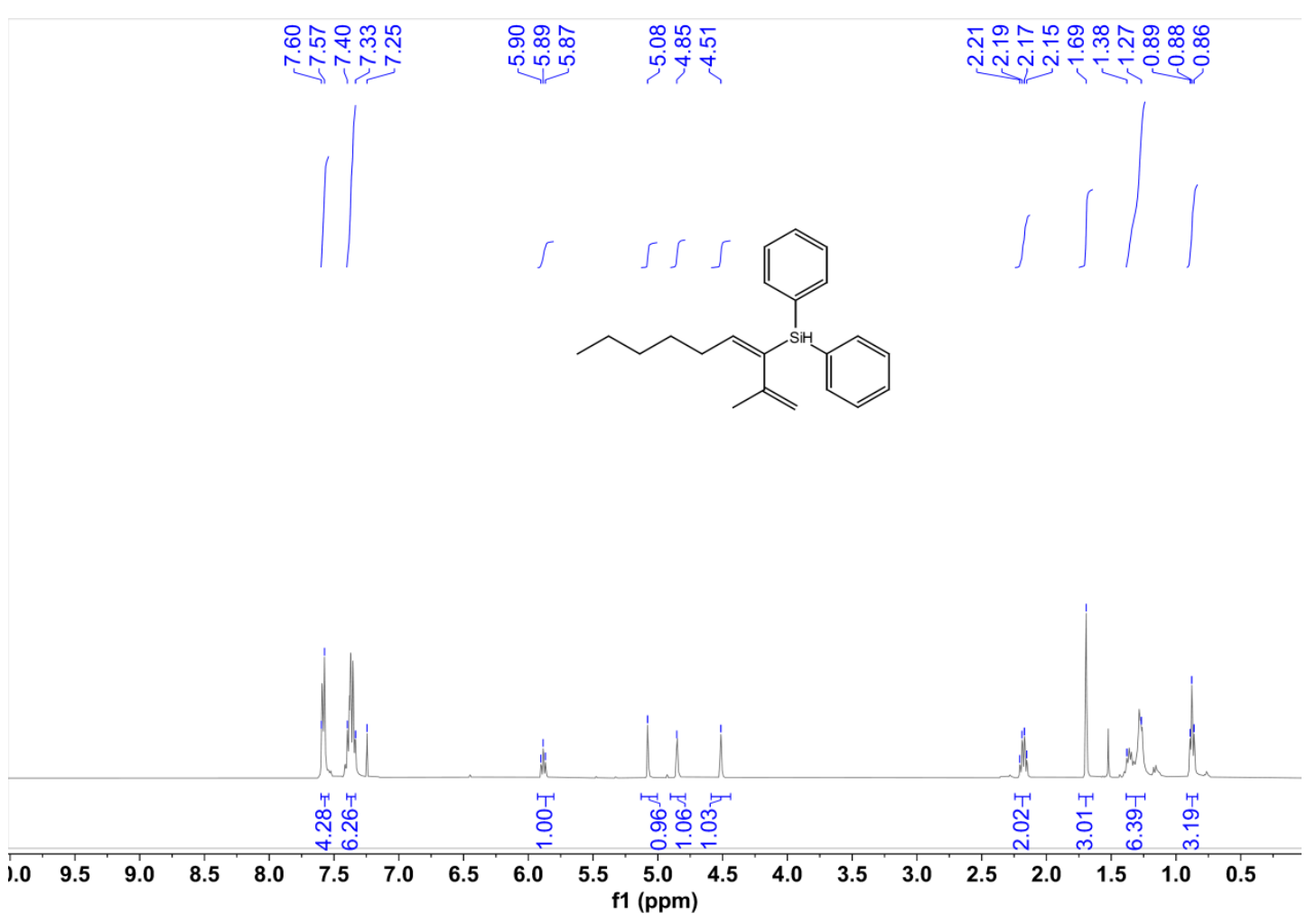

Figure $\mathrm{S93}{ }^{1} \mathrm{H} \mathrm{NMR}\left(\mathrm{CDCl}_{3}, 400 \mathrm{M}\right)$ spectrum of $4 \mathrm{y}^{\prime}$ 

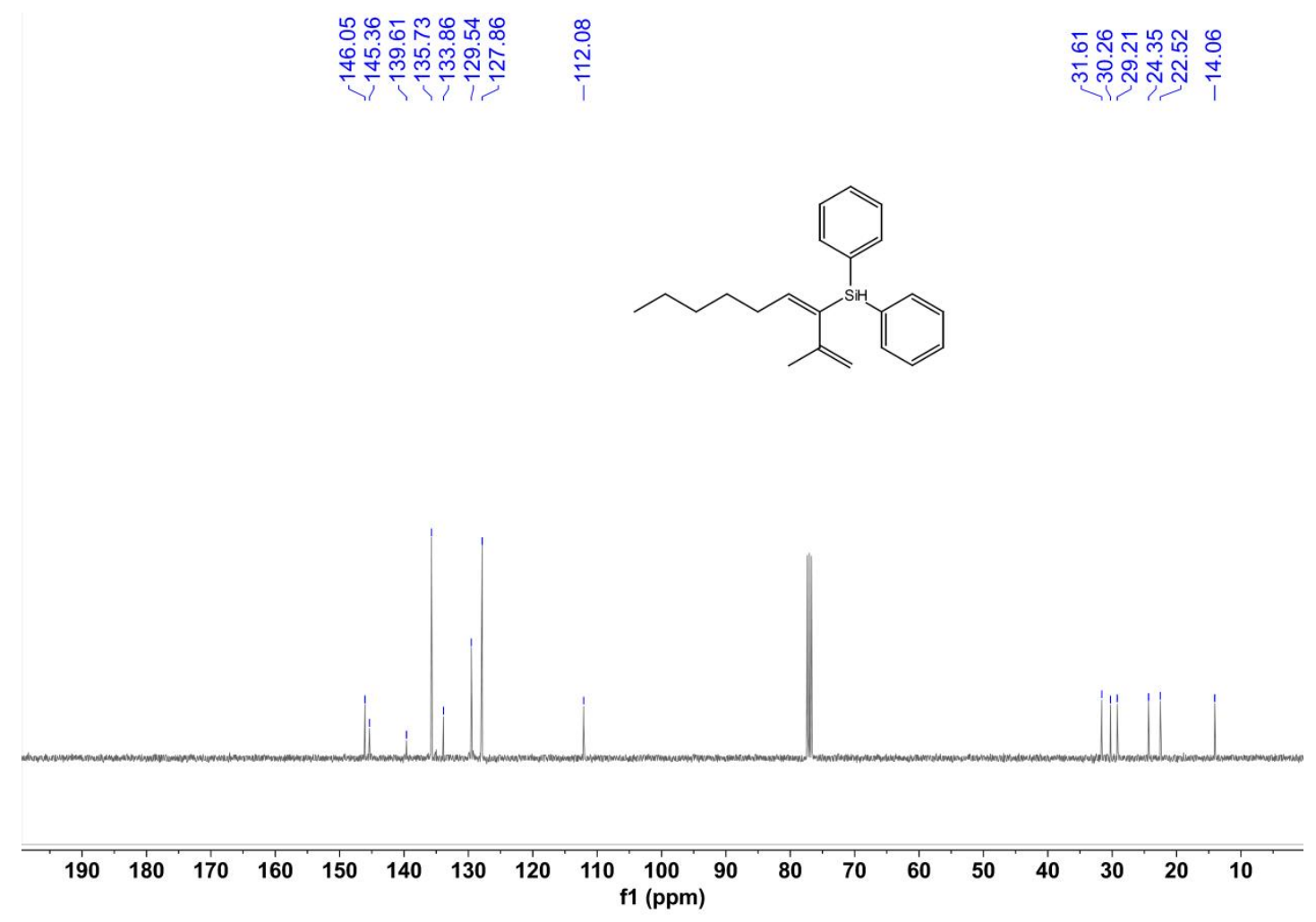

Figure S94 ${ }^{13} \mathrm{C}$ NMR $\left(\mathrm{CDCl}_{3}, 100 \mathrm{M}\right)$ spectrum of $4 \mathrm{y}^{\prime}$

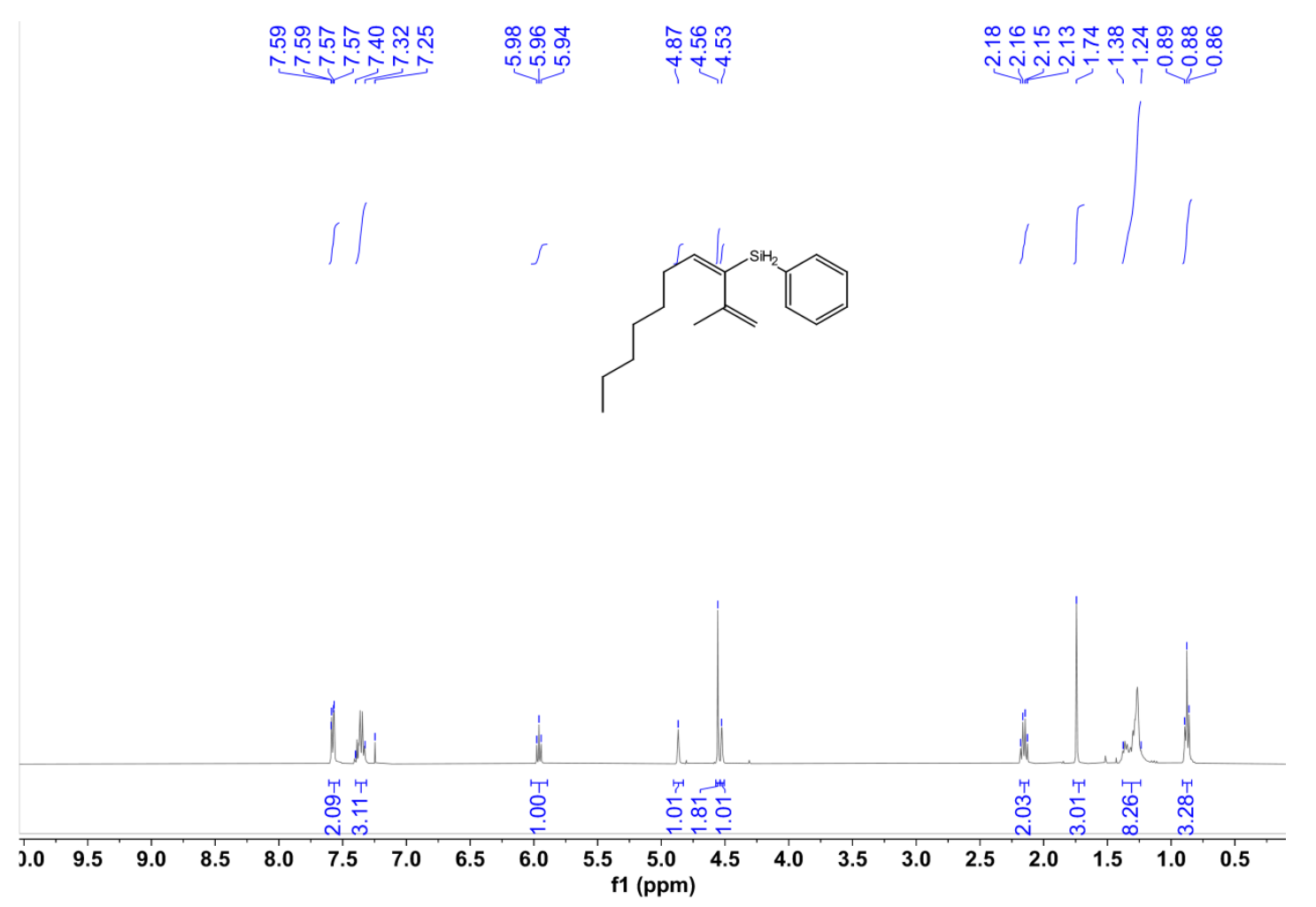

Figure $\mathrm{S95}{ }^{1} \mathrm{H} \mathrm{NMR}\left(\mathrm{CDCl}_{3}, 400 \mathrm{M}\right)$ spectrum of $4 \mathrm{z}$ 

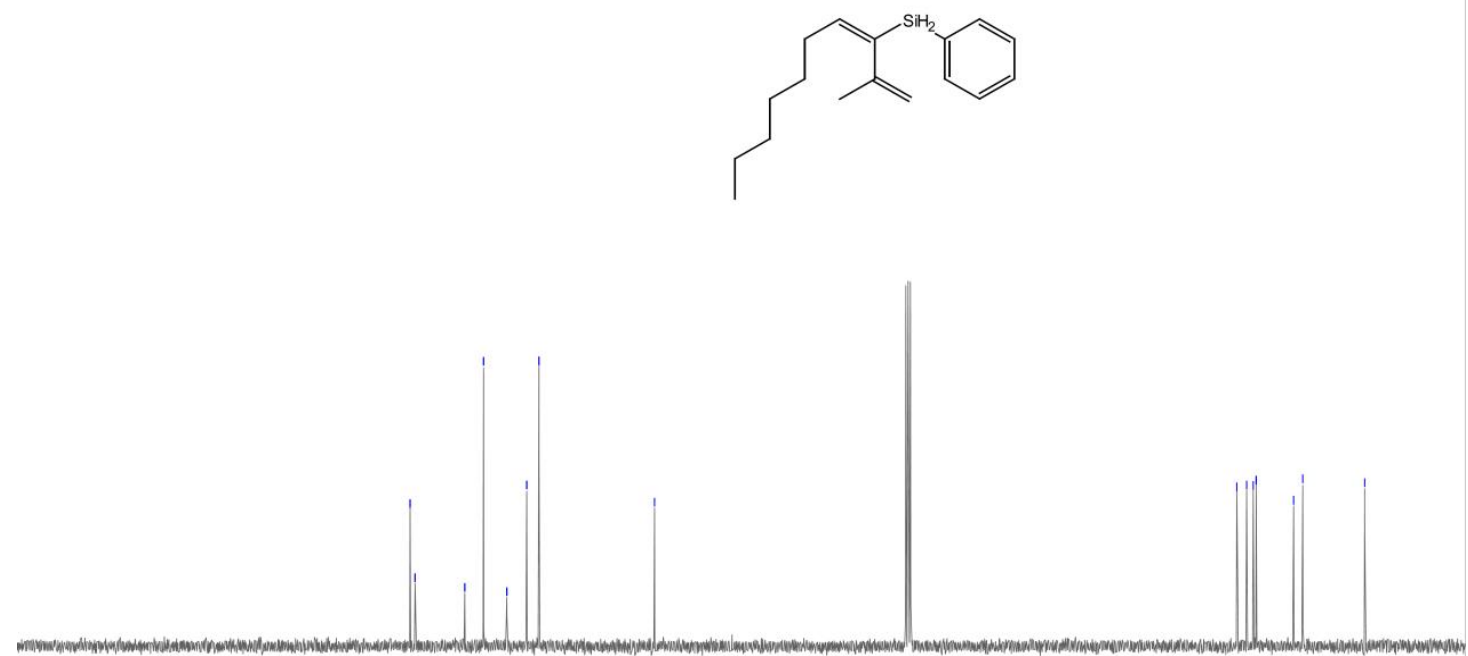

\begin{tabular}{llllllllllllllllllll}
\hline 10 & 190 & 180 & 170 & 160 & 150 & 140 & 130 & 120 & $\begin{array}{c}110 \\
\mathrm{f} 1(\mathrm{ppm})\end{array}$ & 90 & 80 & 70 & 60 & 50 & 40 & 30 & 20 & 10
\end{tabular}

Figure S96 ${ }^{13} \mathrm{C} \mathrm{NMR}\left(\mathrm{CDCl}_{3}, 100 \mathrm{M}\right)$ spectrum of $4 \mathrm{z}$
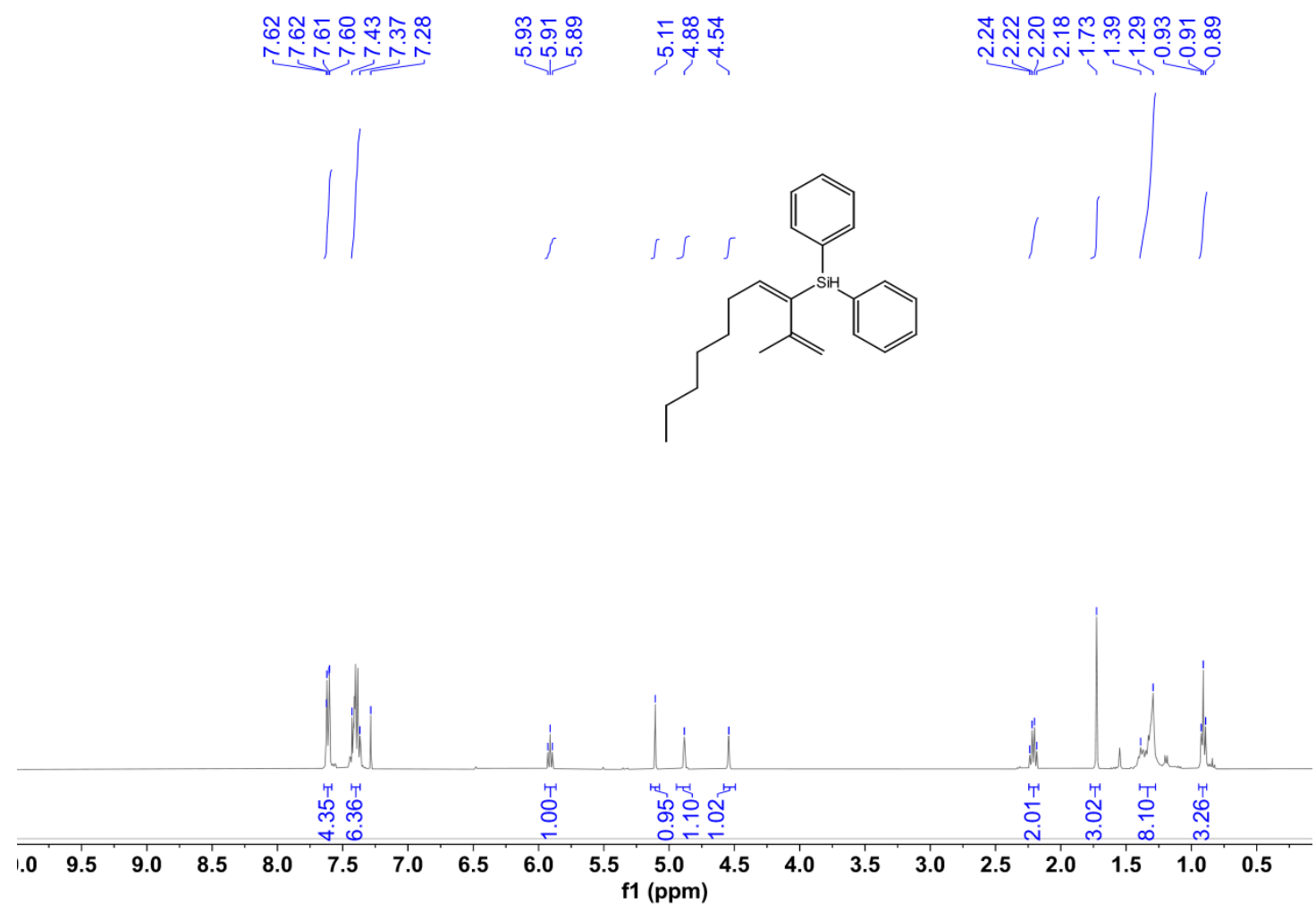

Figure $597{ }^{1} \mathrm{H}$ NMR $\left(\mathrm{CDCl}_{3}, 400 \mathrm{M}\right)$ spectrum of $4 \mathrm{z}^{\prime}$ 


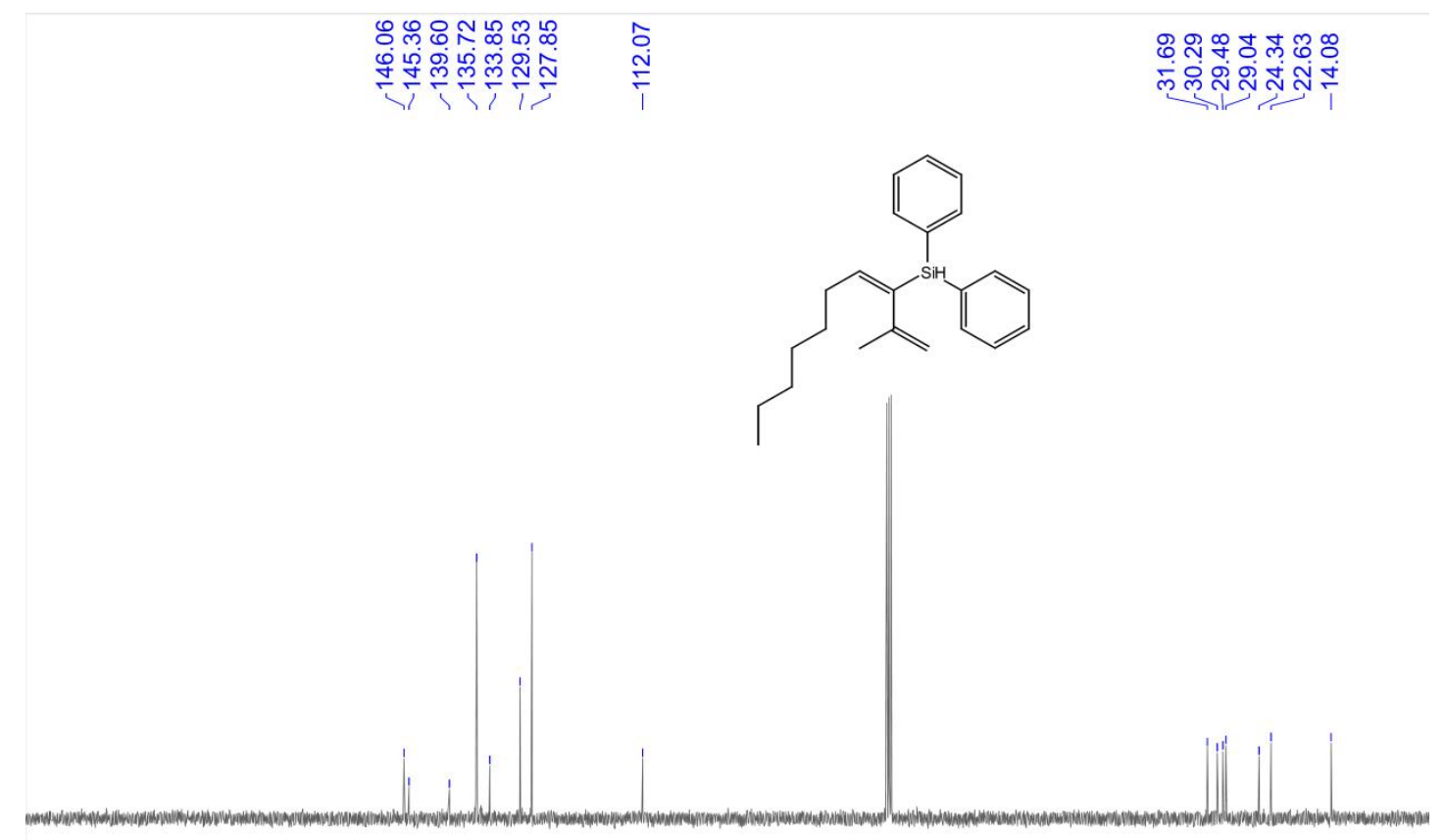

\begin{tabular}{lllllllllllllllllllll}
\hline 10 & 190 & 180 & 170 & 160 & 150 & 140 & 130 & 120 & $110 \begin{array}{l}100 \\
\mathrm{f} 1(\mathrm{ppm})\end{array}$ & 90 & 80 & 70 & 60 & 50 & 40 & 30 & 20 & 10 & 1
\end{tabular}

Figure $\mathrm{S98}{ }^{13} \mathrm{C} \mathrm{NMR}\left(\mathrm{CDCl}_{3}, 100 \mathrm{M}\right)$ spectrum of $4 \mathrm{z}^{\prime}$
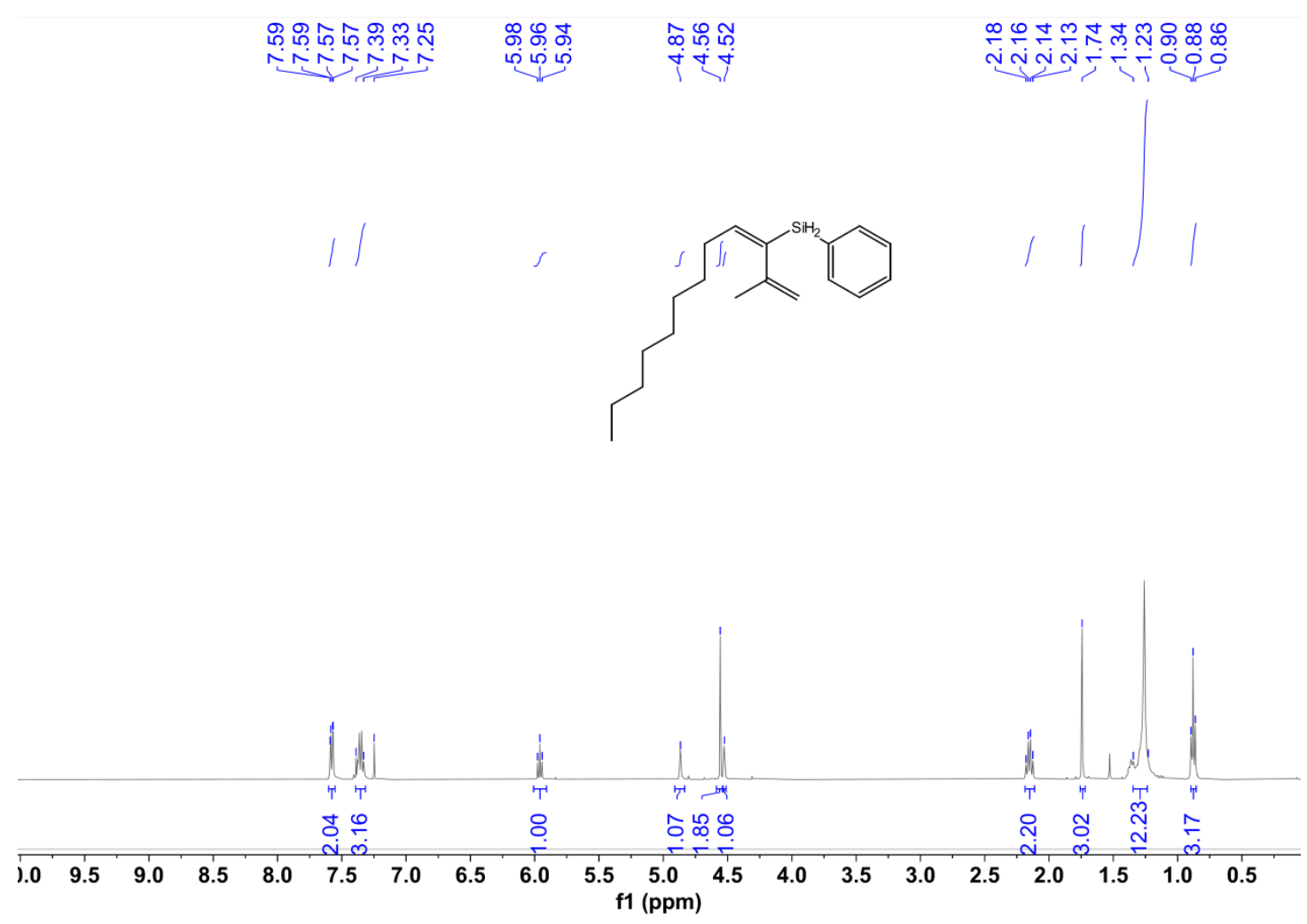

Figure S99 ${ }^{1} \mathrm{H}$ NMR $\left(\mathrm{CDCl}_{3}, 400 \mathrm{M}\right)$ spectrum of $4 \mathrm{aa}$ 


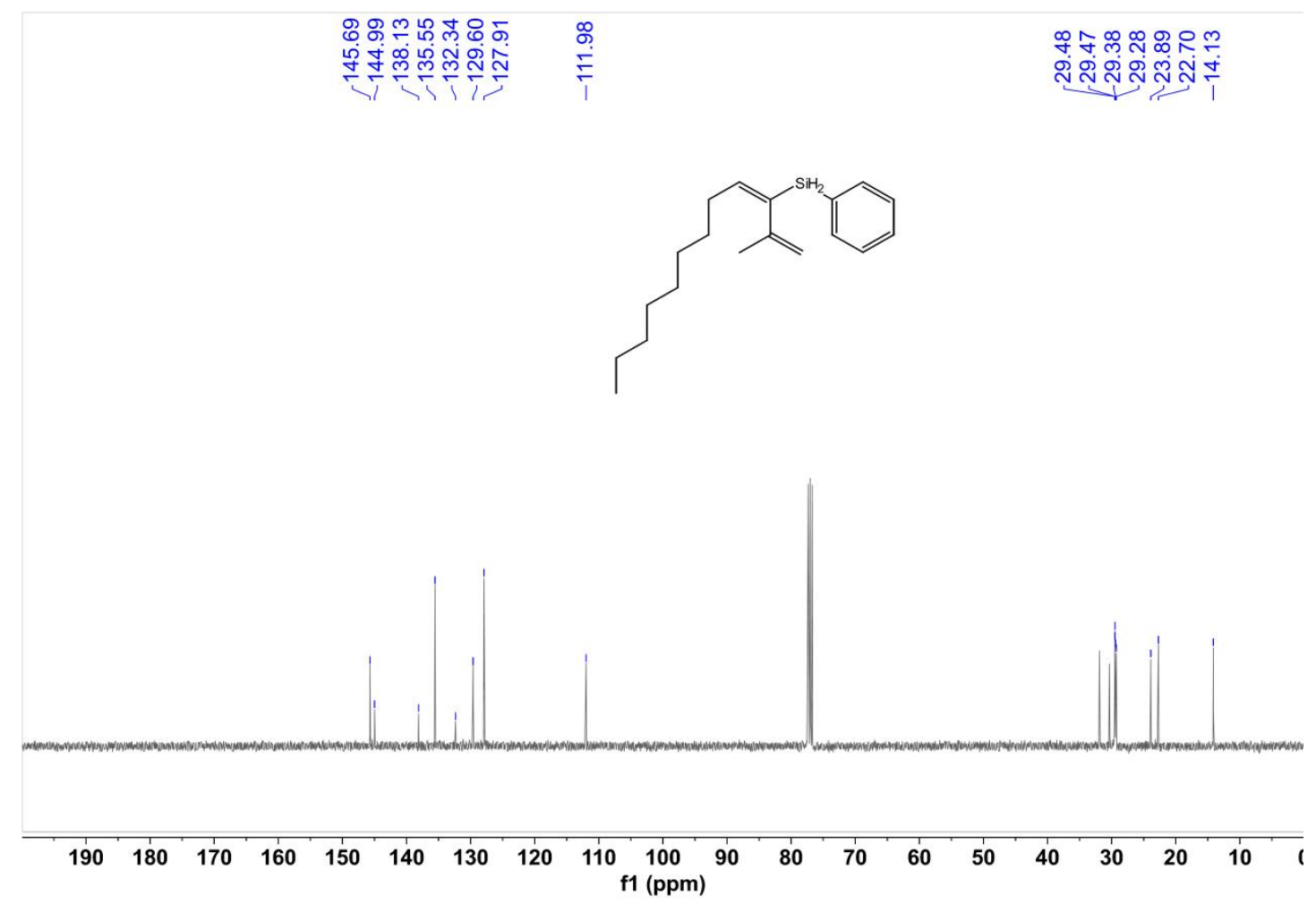

Figure $\mathrm{S100}{ }^{13} \mathrm{C} \mathrm{NMR}\left(\mathrm{CDCl}_{3}, 100 \mathrm{M}\right)$ spectrum of $4 \mathrm{aa}$

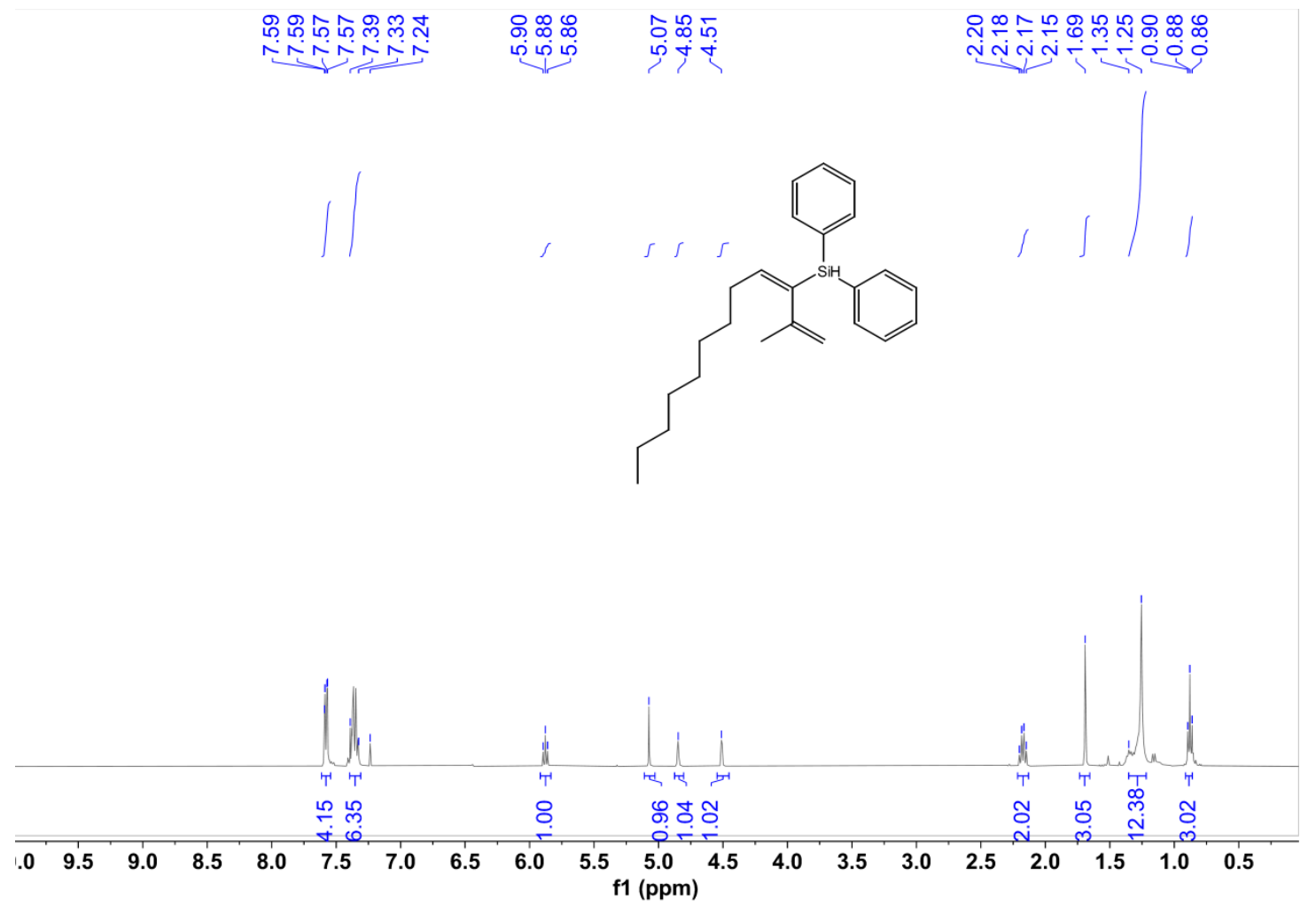

Figure $\mathrm{S101}^{1} \mathrm{H} \mathrm{NMR}\left(\mathrm{CDCl}_{3}, 400 \mathrm{M}\right)$ spectrum of 4aa' 


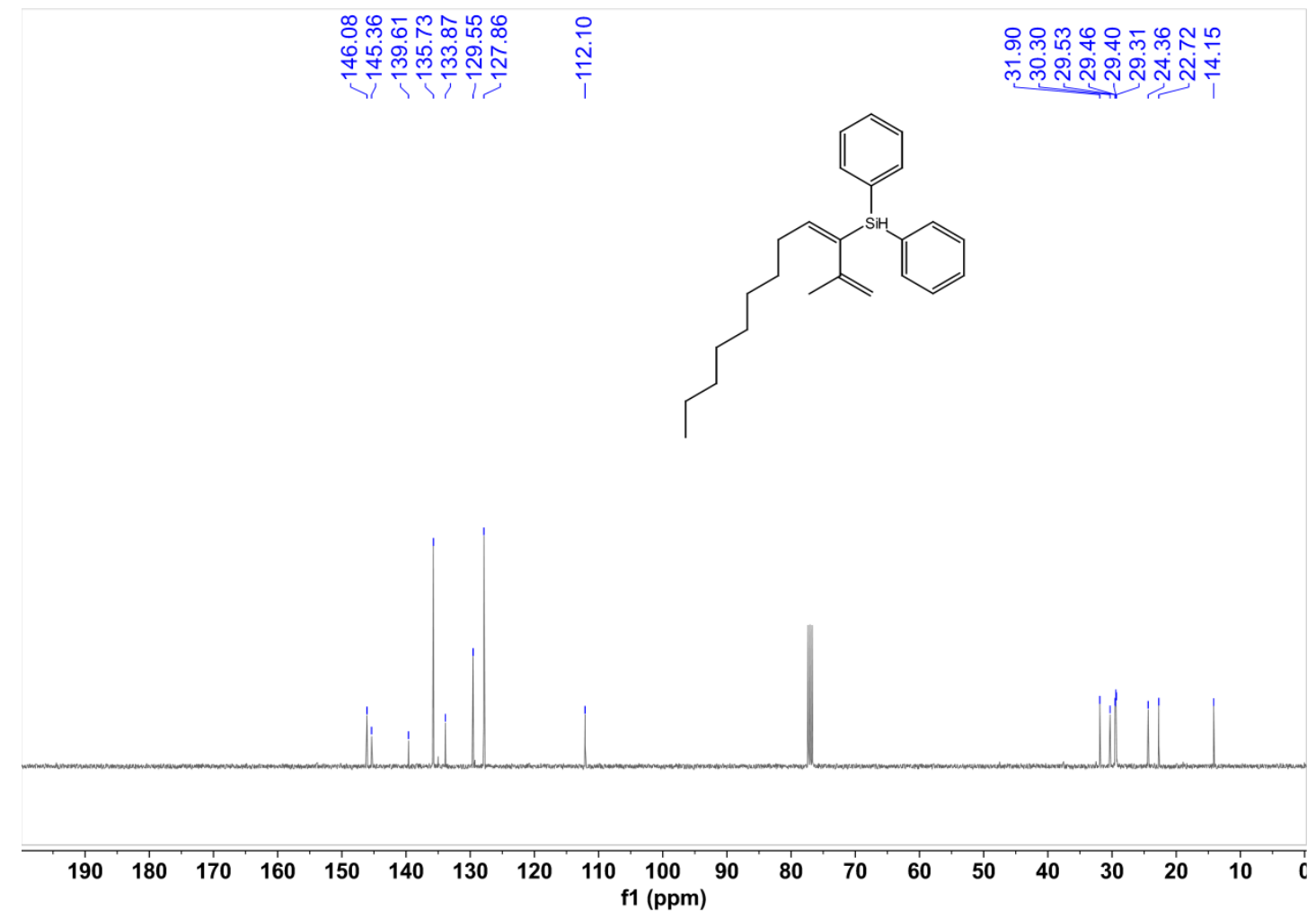

Figure $\mathrm{S102}^{13} \mathrm{C} \mathrm{NMR}\left(\mathrm{CDCl}_{3}, 100 \mathrm{M}\right)$ spectrum of 4aa'

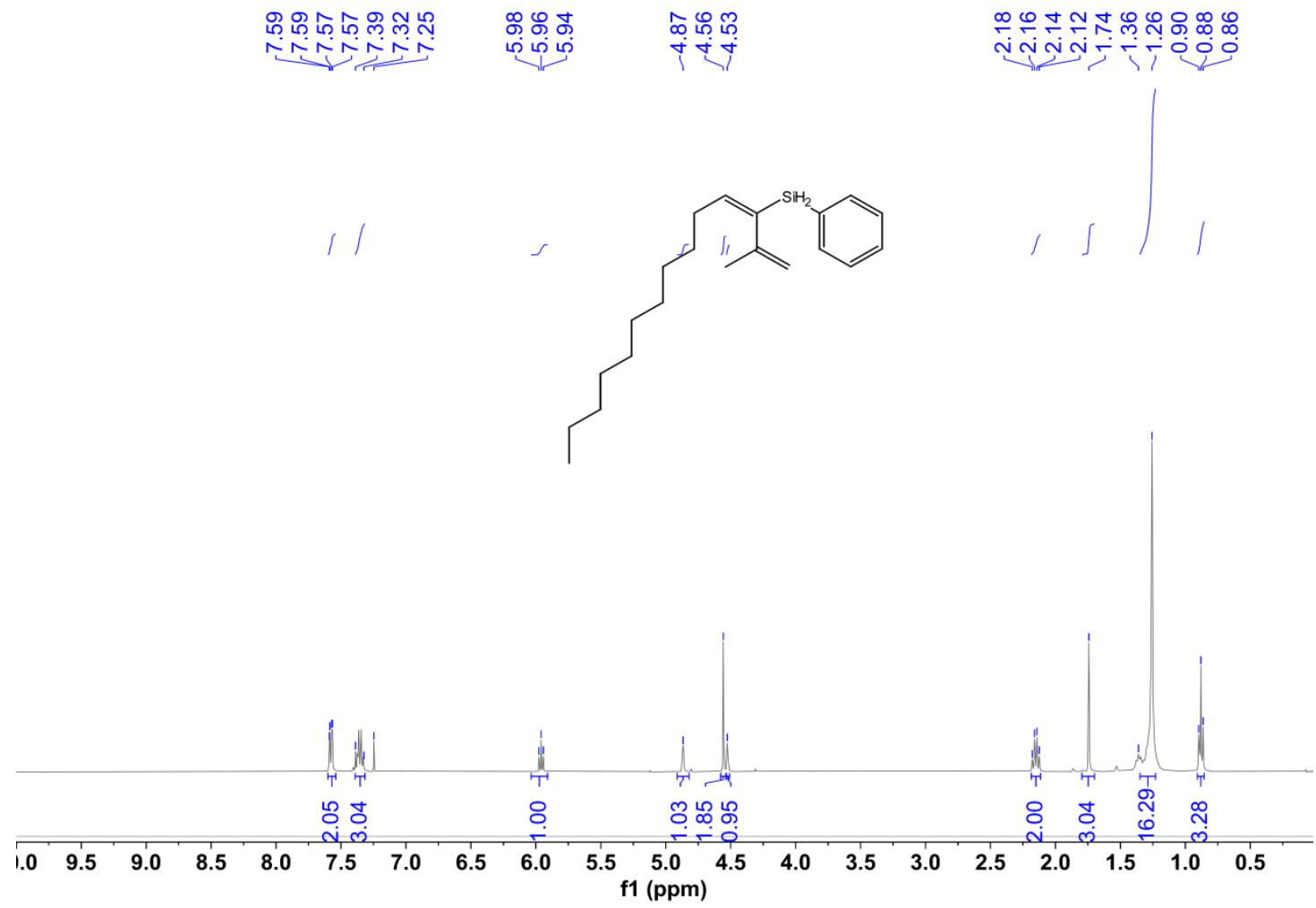

Figure $\mathrm{S103}^{1} \mathrm{H}$ NMR $\left(\mathrm{CDCl}_{3}, 400 \mathrm{M}\right)$ spectrum of $4 \mathrm{ab}$ 

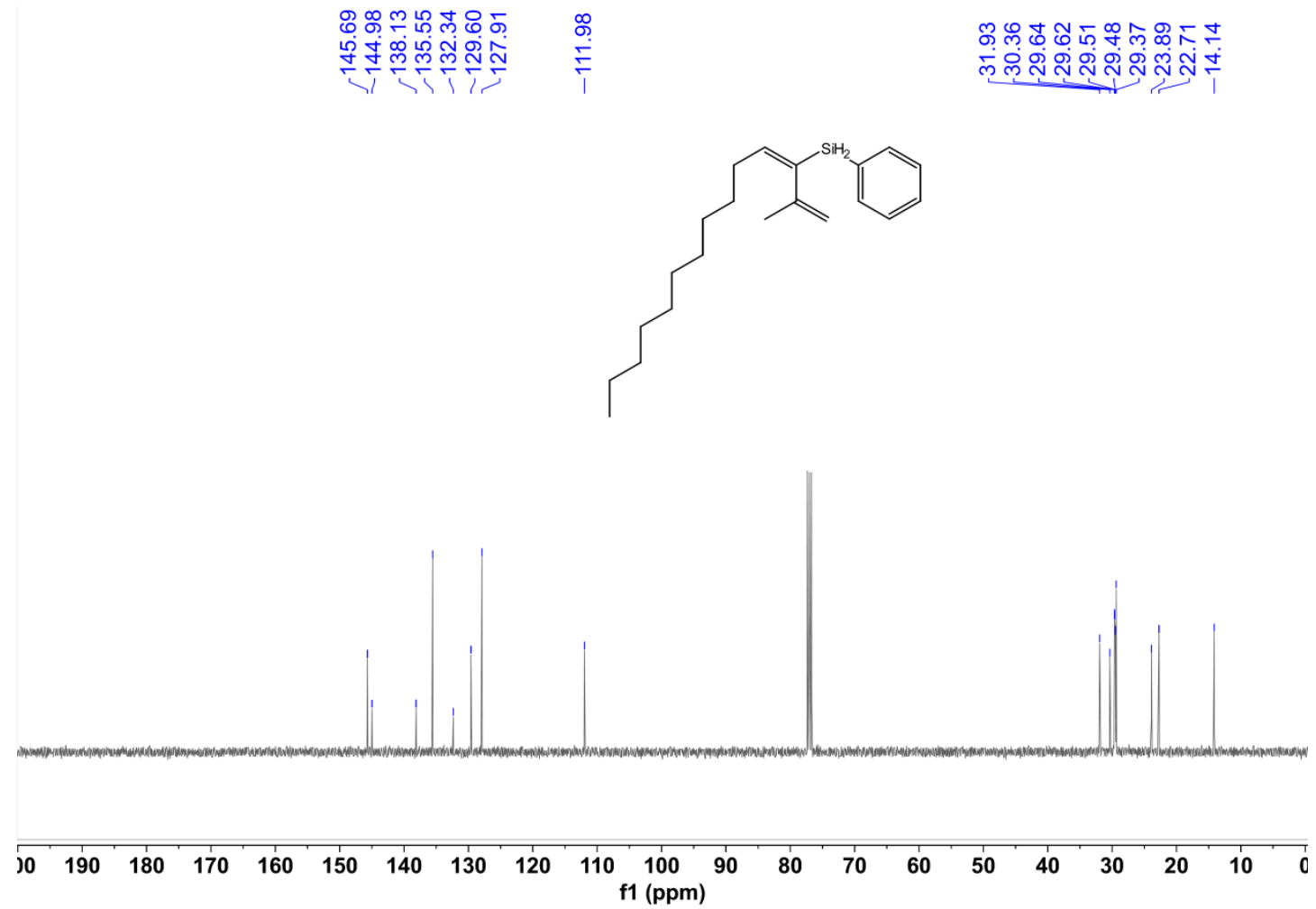

Figure S104 ${ }^{13} \mathrm{C}$ NMR $\left(\mathrm{CDCl}_{3}, 100 \mathrm{M}\right)$ spectrum of $4 \mathrm{ab}$

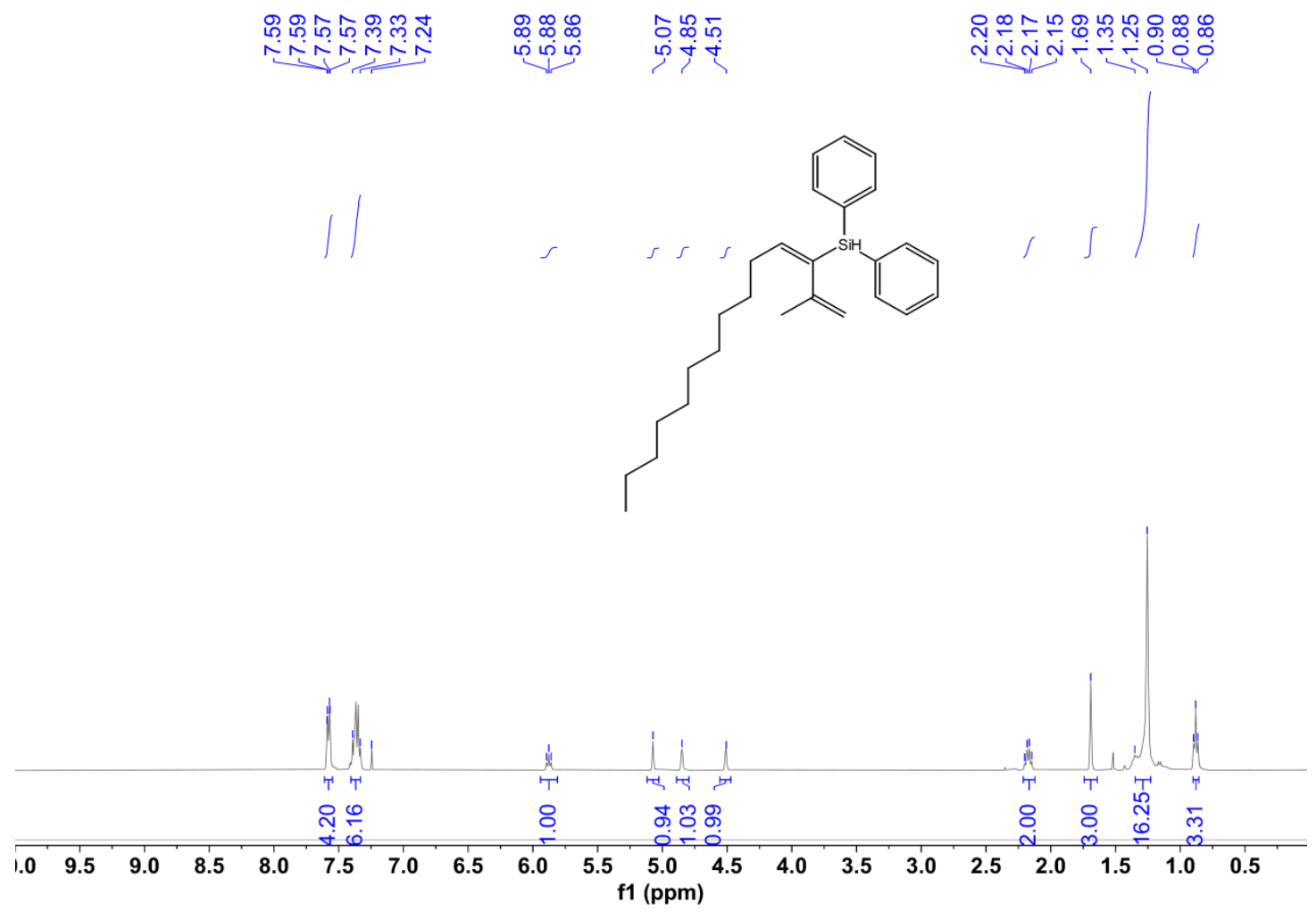

Figure $\mathrm{S105}^{1} \mathrm{H} \mathrm{NMR}\left(\mathrm{CDCl}_{3}, 400 \mathrm{M}\right)$ spectrum of 4ab' 


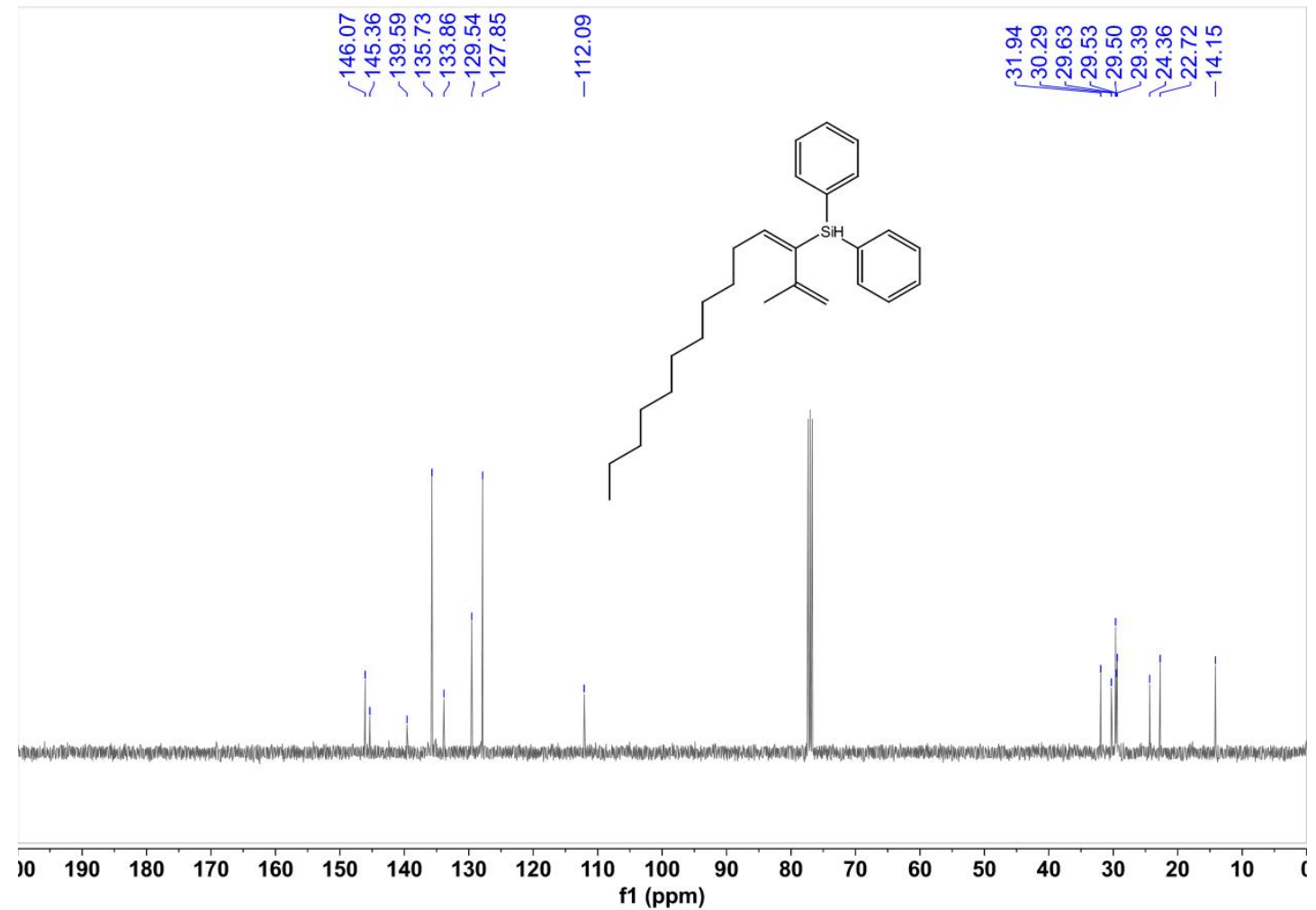

Figure $\mathrm{S106}^{13} \mathrm{C} \mathrm{NMR}\left(\mathrm{CDCl}_{3}, 100 \mathrm{M}\right)$ spectrum of 4ab'
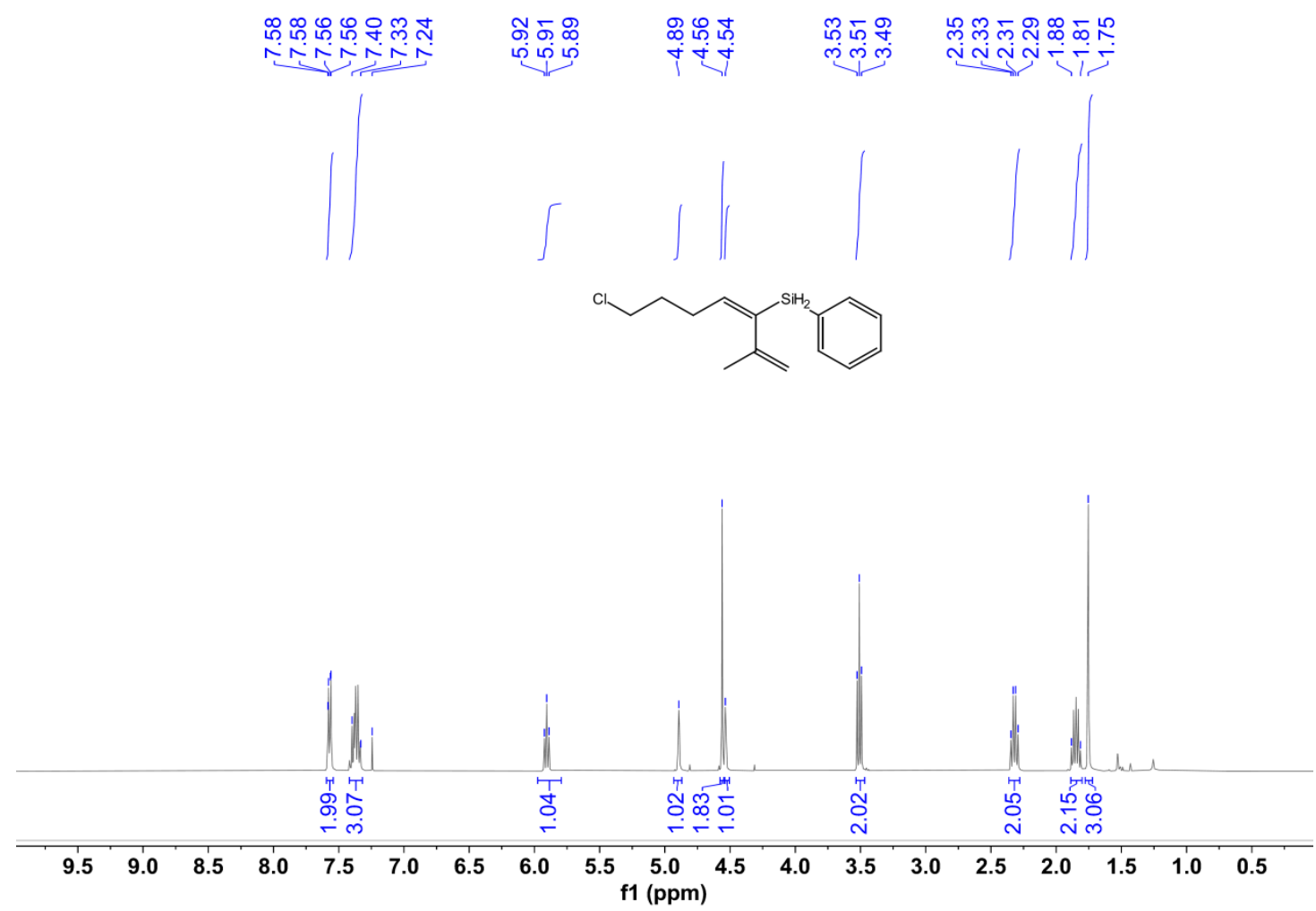

Figure $\mathrm{S107}{ }^{1} \mathrm{H}$ NMR $\left(\mathrm{CDCl}_{3}, 400 \mathrm{M}\right)$ spectrum of $4 \mathrm{ac}$ 


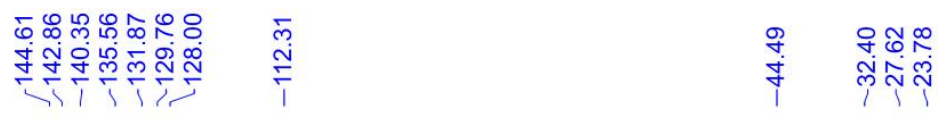
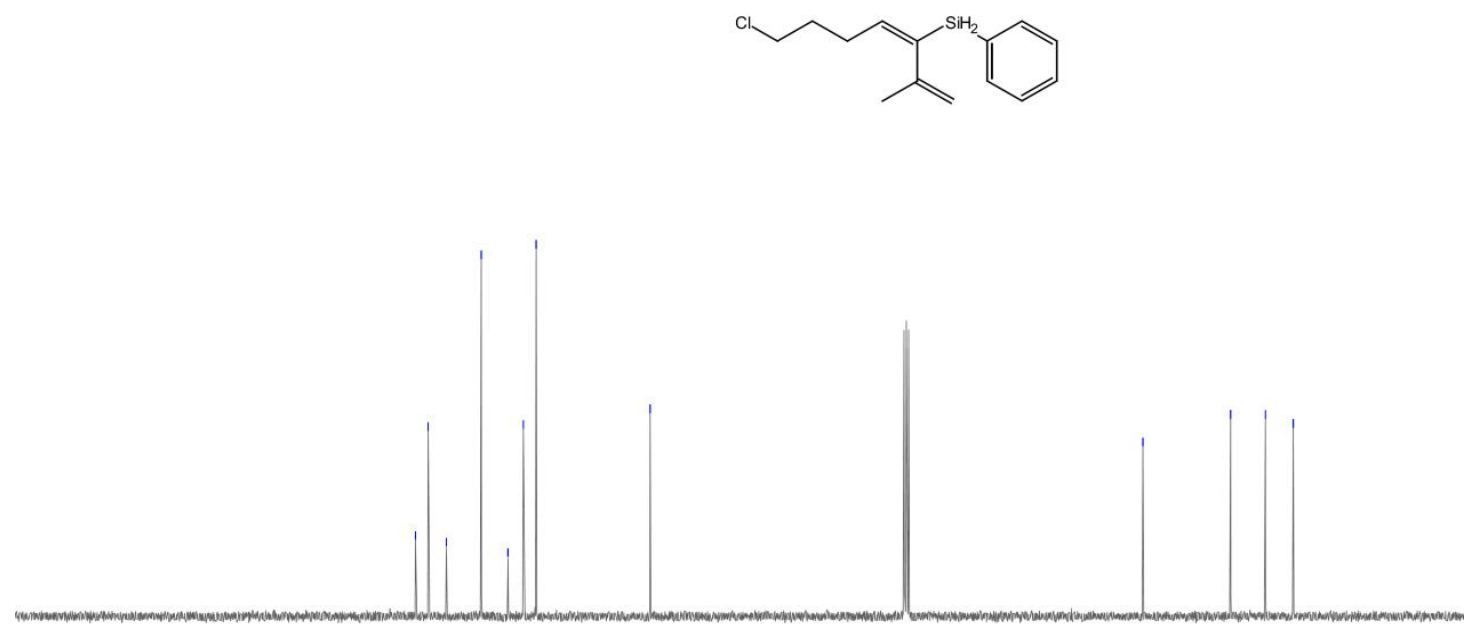

$\begin{array}{lllllllllllllllllllllllll}190 & 180 & 170 & 160 & 150 & 140 & 130 & 120 & 110 & 100 & 90 & 80 & 70 & 60 & 50 & 40 & 30 & 20 & 10\end{array}$

Figure $\mathrm{S108}^{13} \mathrm{C}$ NMR $\left(\mathrm{CDCl}_{3}, 100 \mathrm{M}\right)$ spectrum of $4 \mathrm{ac}$
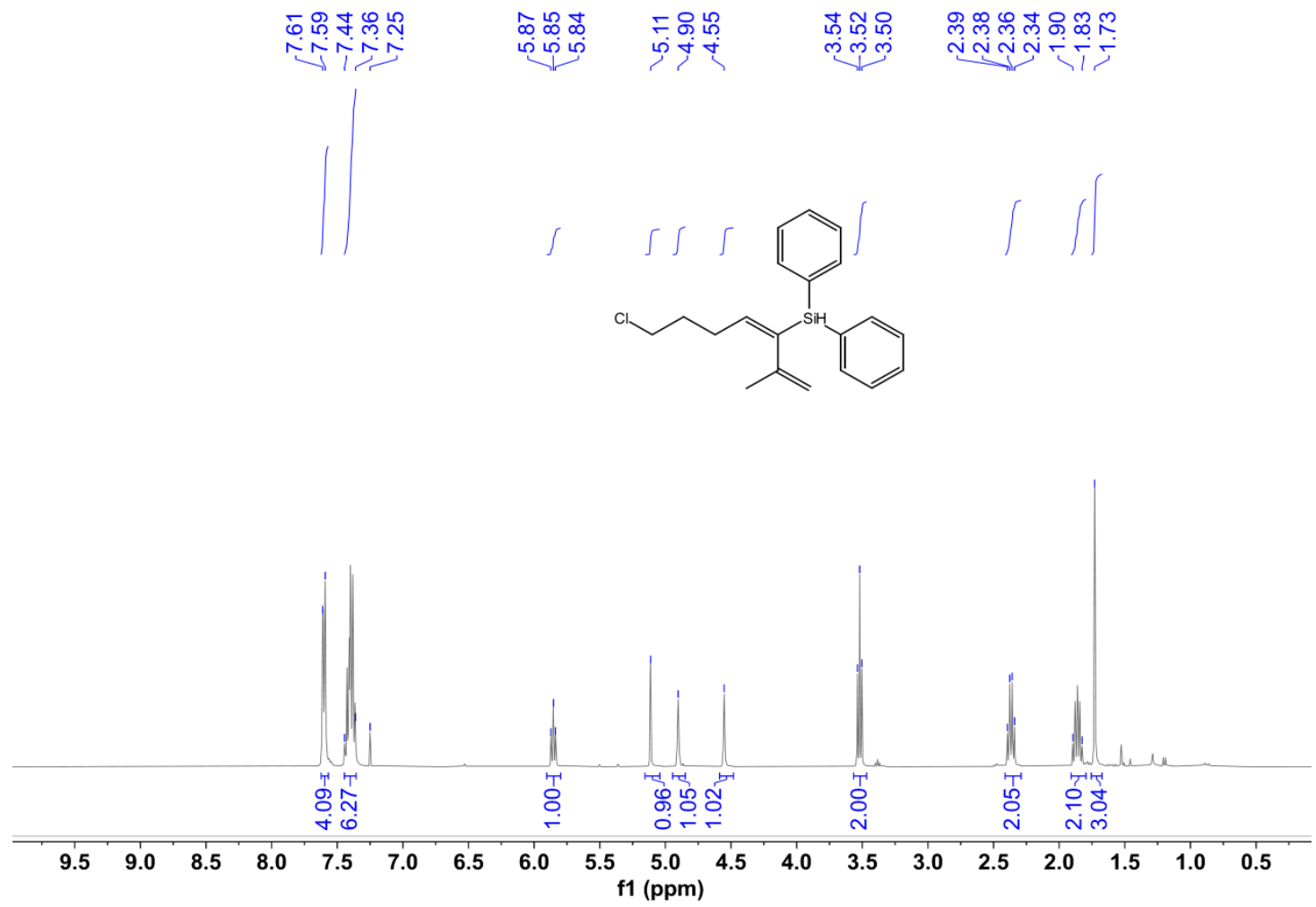

Figure $\mathrm{S109}{ }^{1} \mathrm{H}$ NMR $\left(\mathrm{CDCl}_{3}, 400 \mathrm{M}\right)$ spectrum of $4 \mathrm{ac}^{\prime}$ 


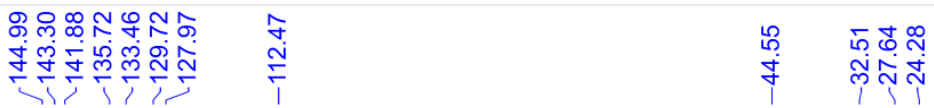

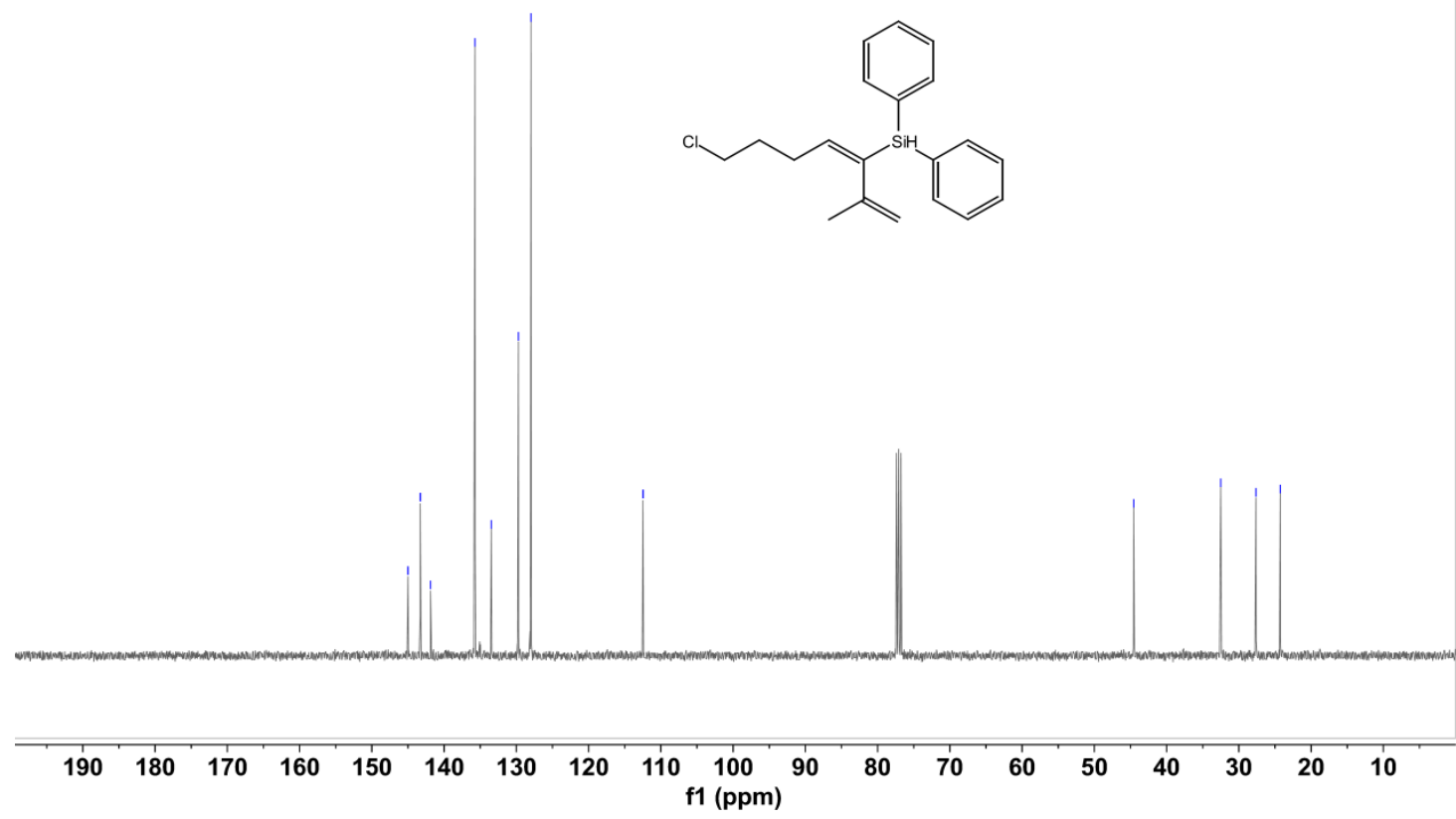

Figure $\mathrm{S110}{ }^{13} \mathrm{C}$ NMR $\left(\mathrm{CDCl}_{3}, 100 \mathrm{M}\right)$ spectrum of $4 \mathrm{ac}^{\prime}$
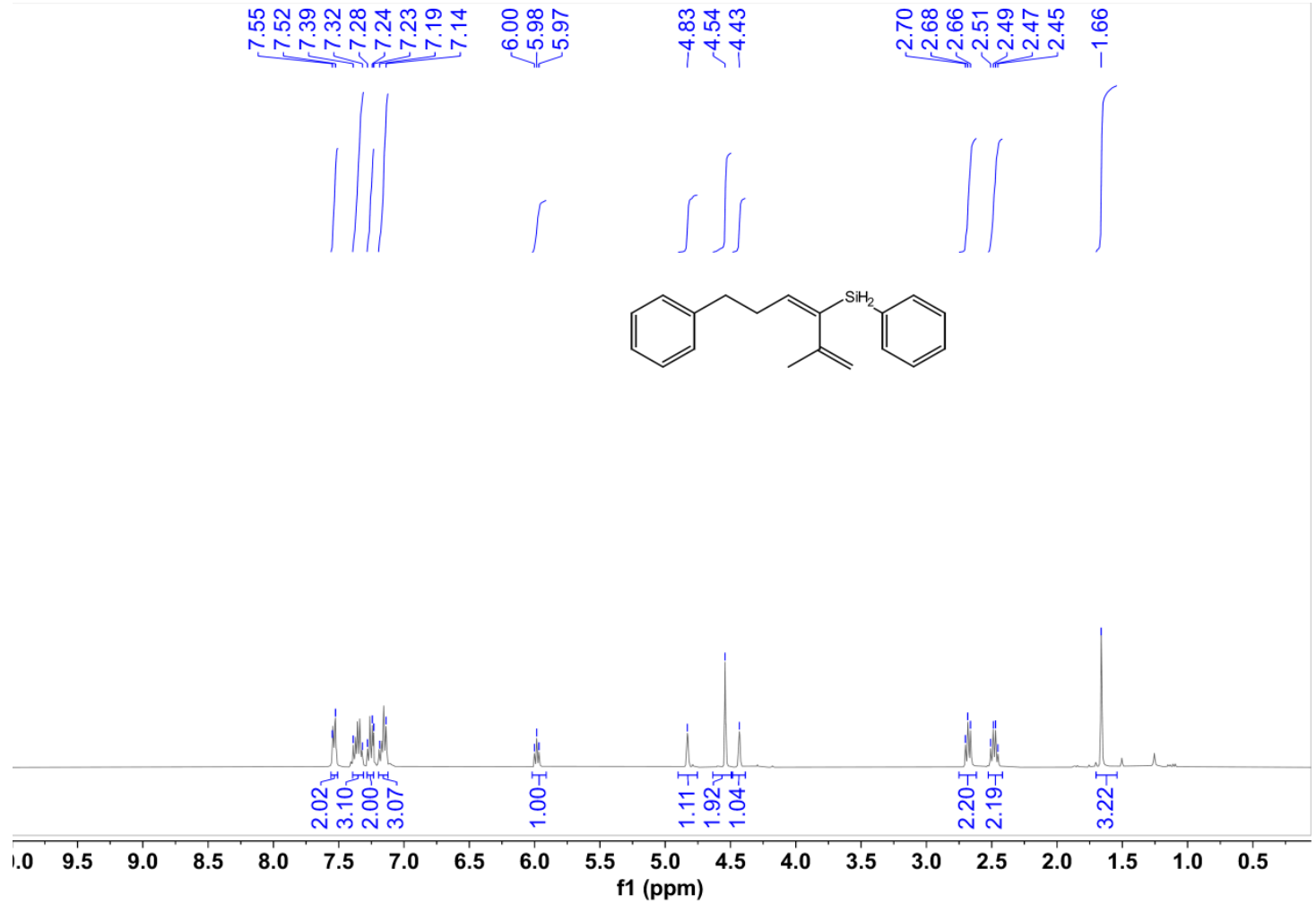

Figure $\mathrm{S111}{ }^{1} \mathrm{H}$ NMR $\left(\mathrm{CDCl}_{3}, 400 \mathrm{M}\right)$ spectrum of $4 \mathrm{ad}$ 

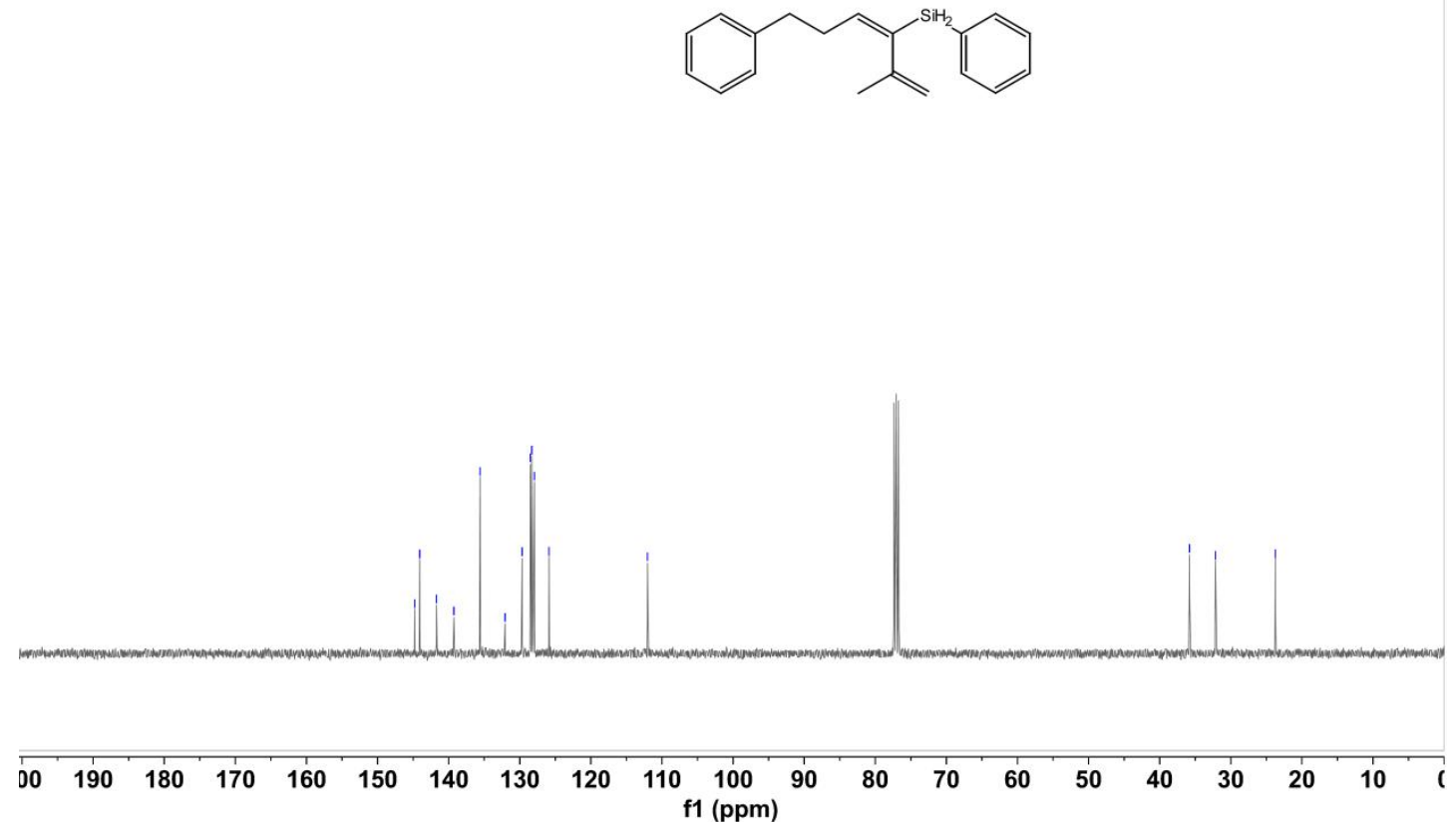

Figure $\mathrm{S}_{112}{ }^{13} \mathrm{C} \mathrm{NMR}\left(\mathrm{CDCl}_{3}, 100 \mathrm{M}\right)$ spectrum of $4 \mathrm{ad}$
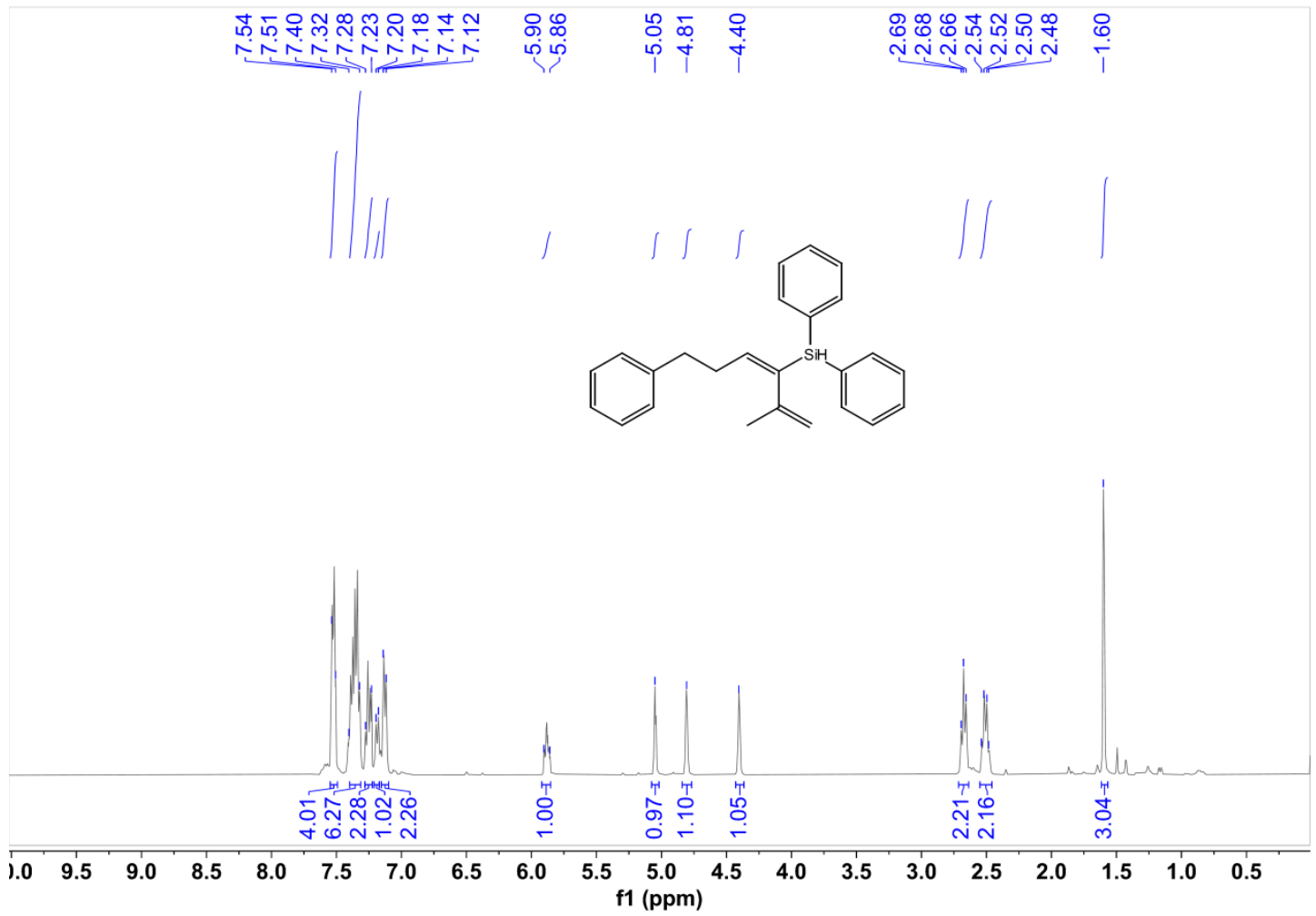

Figure $\mathrm{S113}^{1} \mathrm{H} \mathrm{NMR}\left(\mathrm{CDCl}_{3}, 400 \mathrm{M}\right)$ spectrum of 4ad' 
<smiles>C=C(C)/C(=C\CCc1ccccc1)[SH](c1ccccc1)c1ccccc1</smiles>

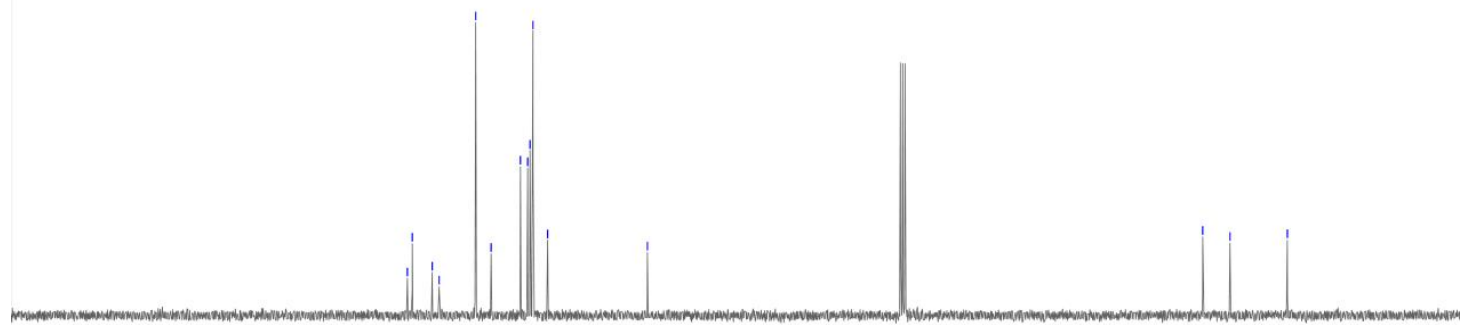

$\begin{array}{llllllllllllllllllllll}190 & 180 & 170 & 160 & 150 & 140 & 130 & 120 & 110 \begin{array}{c}100 \\ \mathrm{f} 1(\mathrm{ppm})\end{array} & 90 & 80 & 70 & 60 & 50 & 40 & 30 & 20 & 10\end{array}$

Figure $\mathrm{S114}{ }^{13} \mathrm{C} \mathrm{NMR}\left(\mathrm{CDCl}_{3}, 100 \mathrm{M}\right)$ spectrum of 4ad'

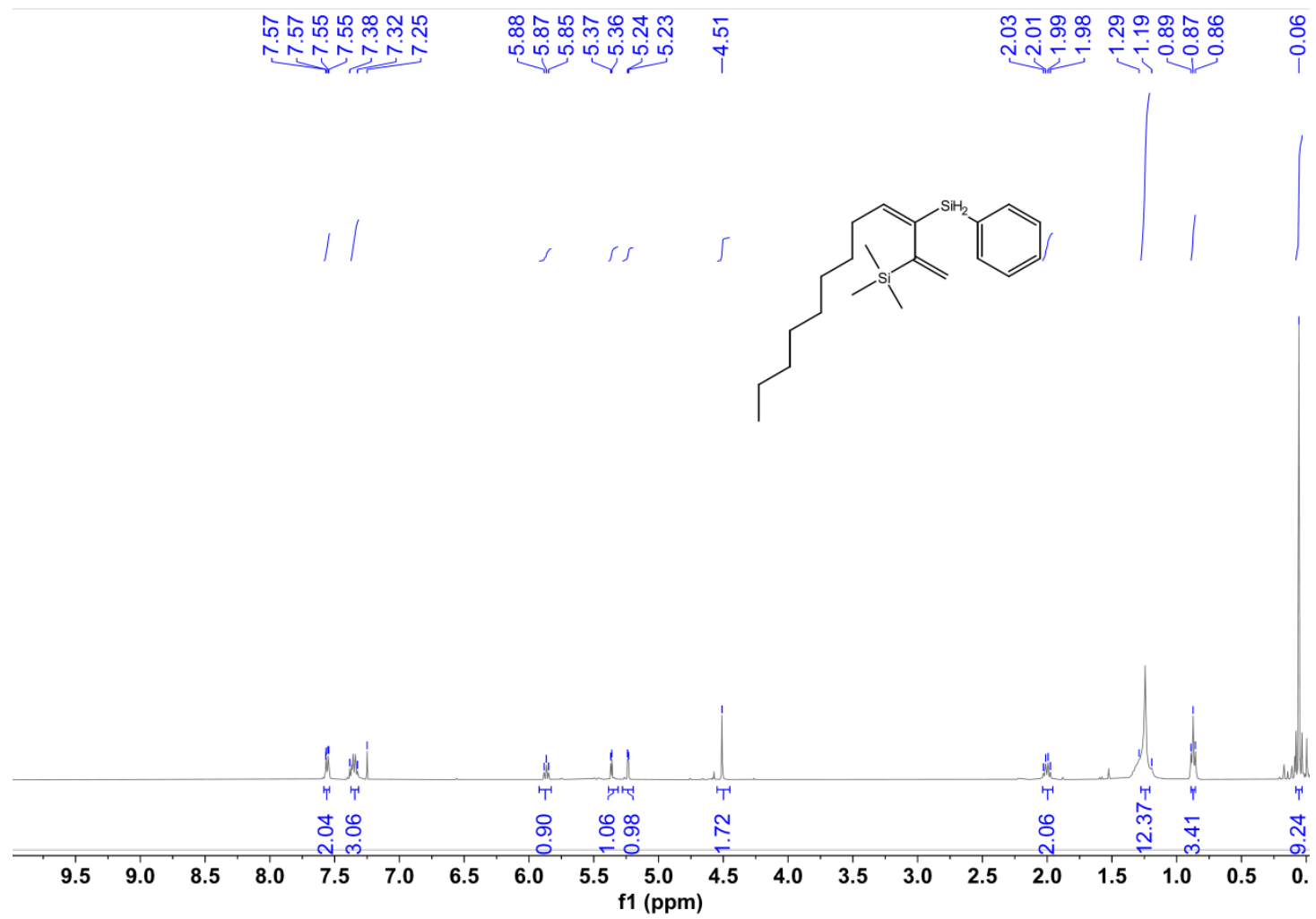

Figure $\mathrm{S115}^{1} \mathrm{H}$ NMR $\left(\mathrm{CDCl}_{3}, 400 \mathrm{M}\right)$ spectrum of $4 \mathrm{ae}$ 


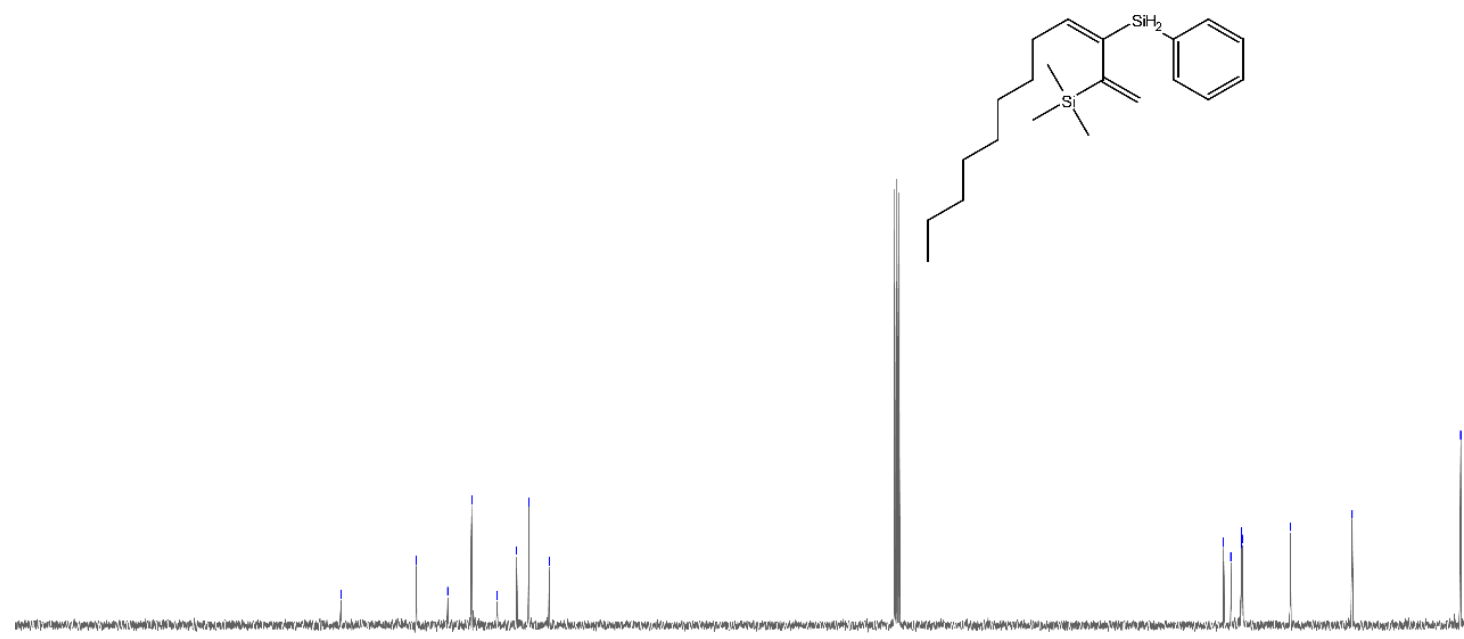

\begin{tabular}{lllllllllllllllllllllllllllll}
\hline 10 & 190 & 180 & 170 & 160 & 150 & 140 & 130 & 120 & 110 & 100 & 90 & 80 & 70 & 60 & 50 & 40 & 30 & 20 & 10 & 0
\end{tabular}

Figure $\mathrm{S116}^{13} \mathrm{C} \mathrm{NMR}\left(\mathrm{CDCl}_{3}, 100 \mathrm{M}\right)$ spectrum of $4 \mathrm{ae}$

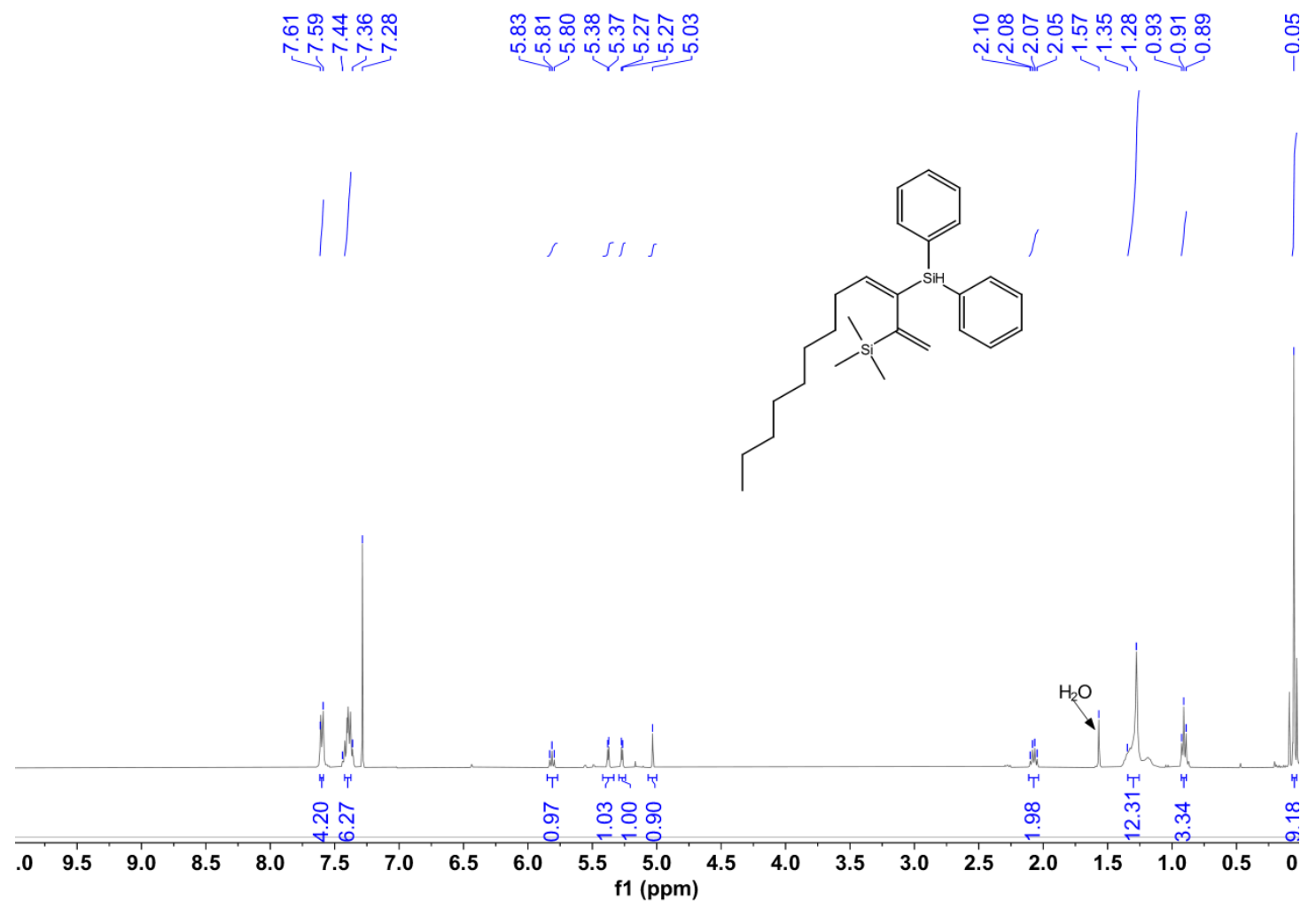

Figure $\mathrm{S117}^{1} \mathrm{H}$ NMR $\left(\mathrm{CDCl}_{3}, 400 \mathrm{M}\right)$ spectrum of $4 \mathrm{ae}^{\prime}$ 


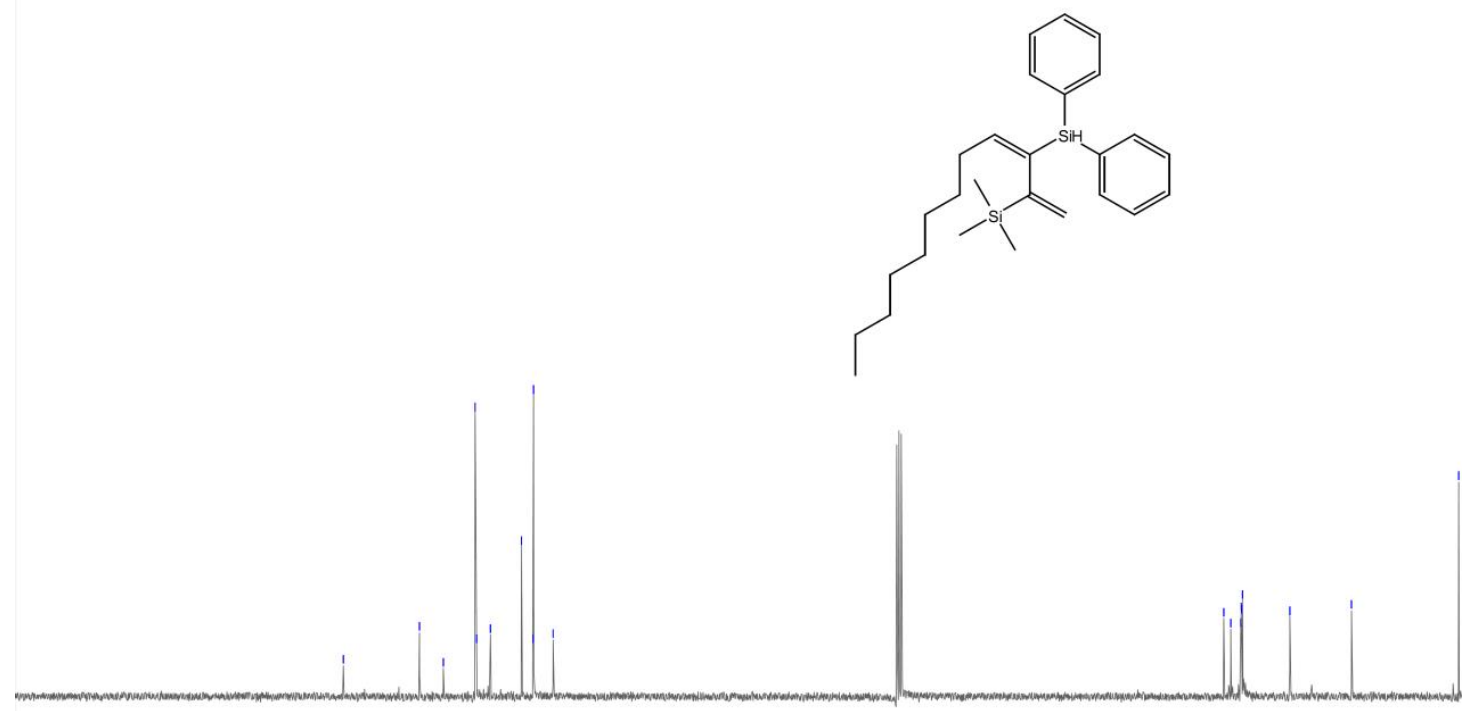

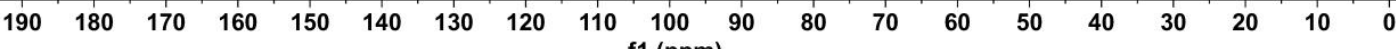

Figure $\mathrm{S118}^{13} \mathrm{C}$ NMR $\left(\mathrm{CDCl}_{3}, 100 \mathrm{M}\right)$ spectrum of $4 \mathrm{ae}^{\prime}$

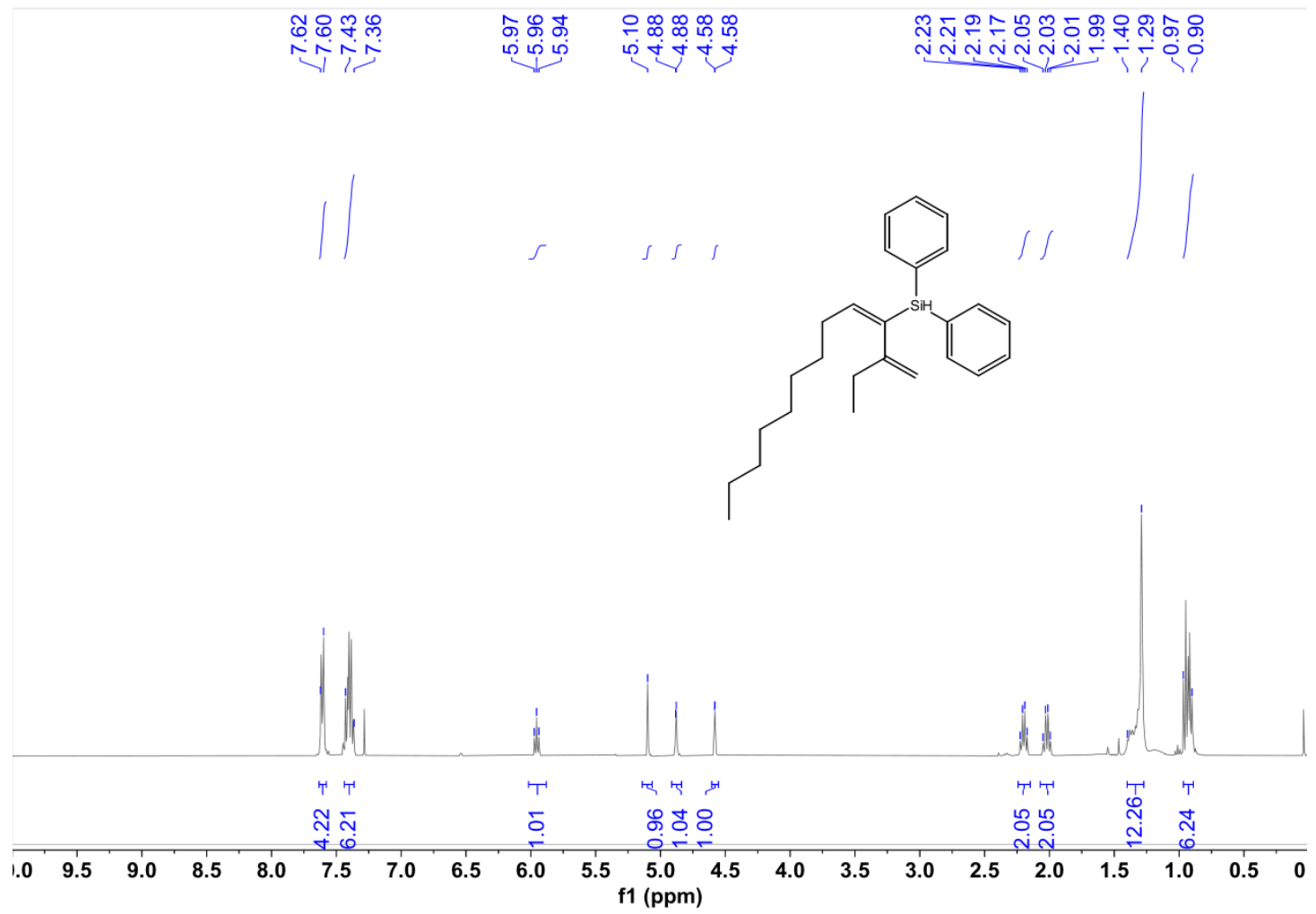

Figure $\mathrm{S119}{ }^{1} \mathrm{H} \mathrm{NMR}\left(\mathrm{CDCl}_{3}, 400 \mathrm{M}\right)$ spectrum of $4 \mathrm{af}^{\prime}$ 

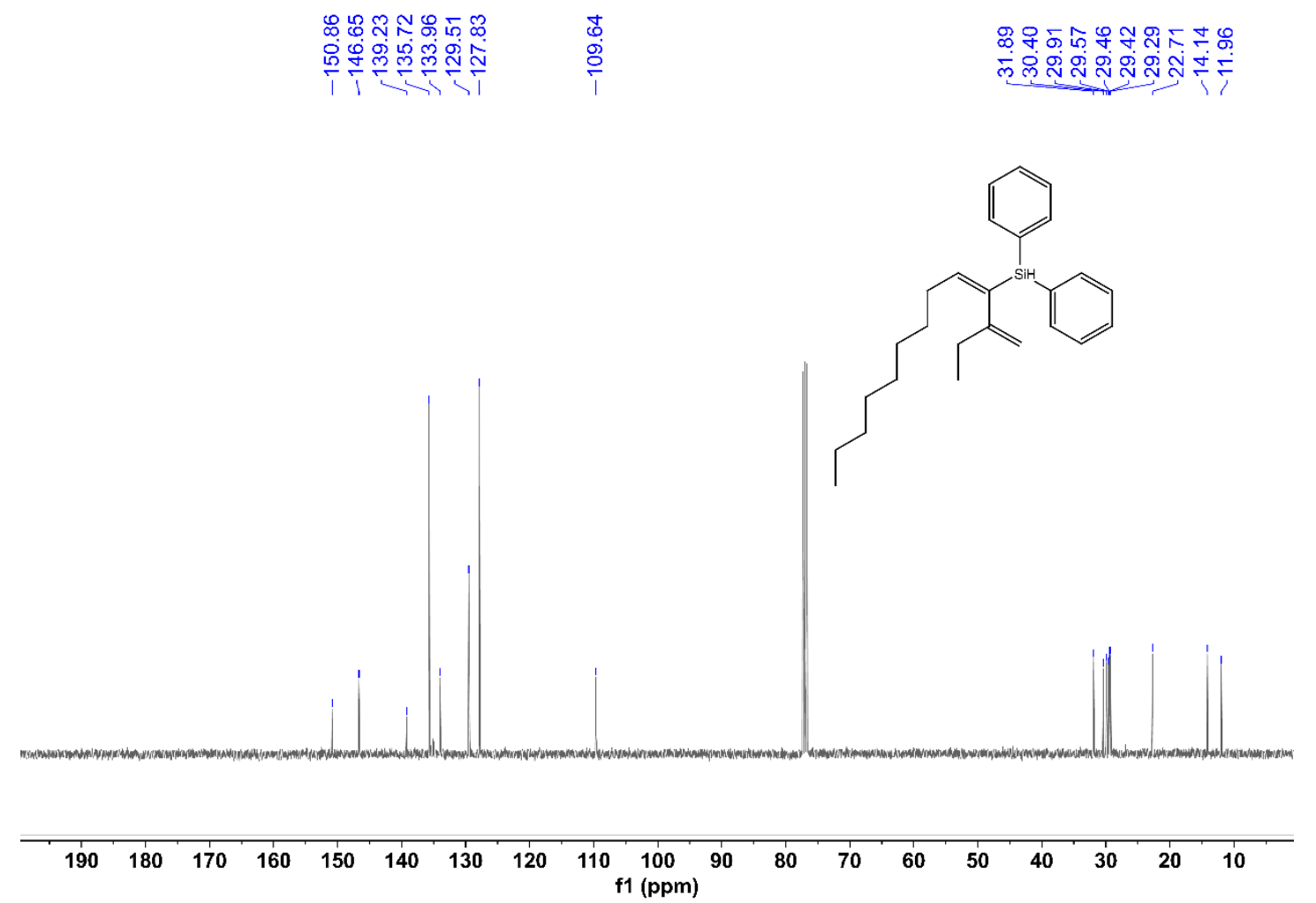

Figure $\mathrm{S120}^{13} \mathrm{C} \mathrm{NMR}\left(\mathrm{CDCl}_{3}, 100 \mathrm{M}\right)$ spectrum of 4af'

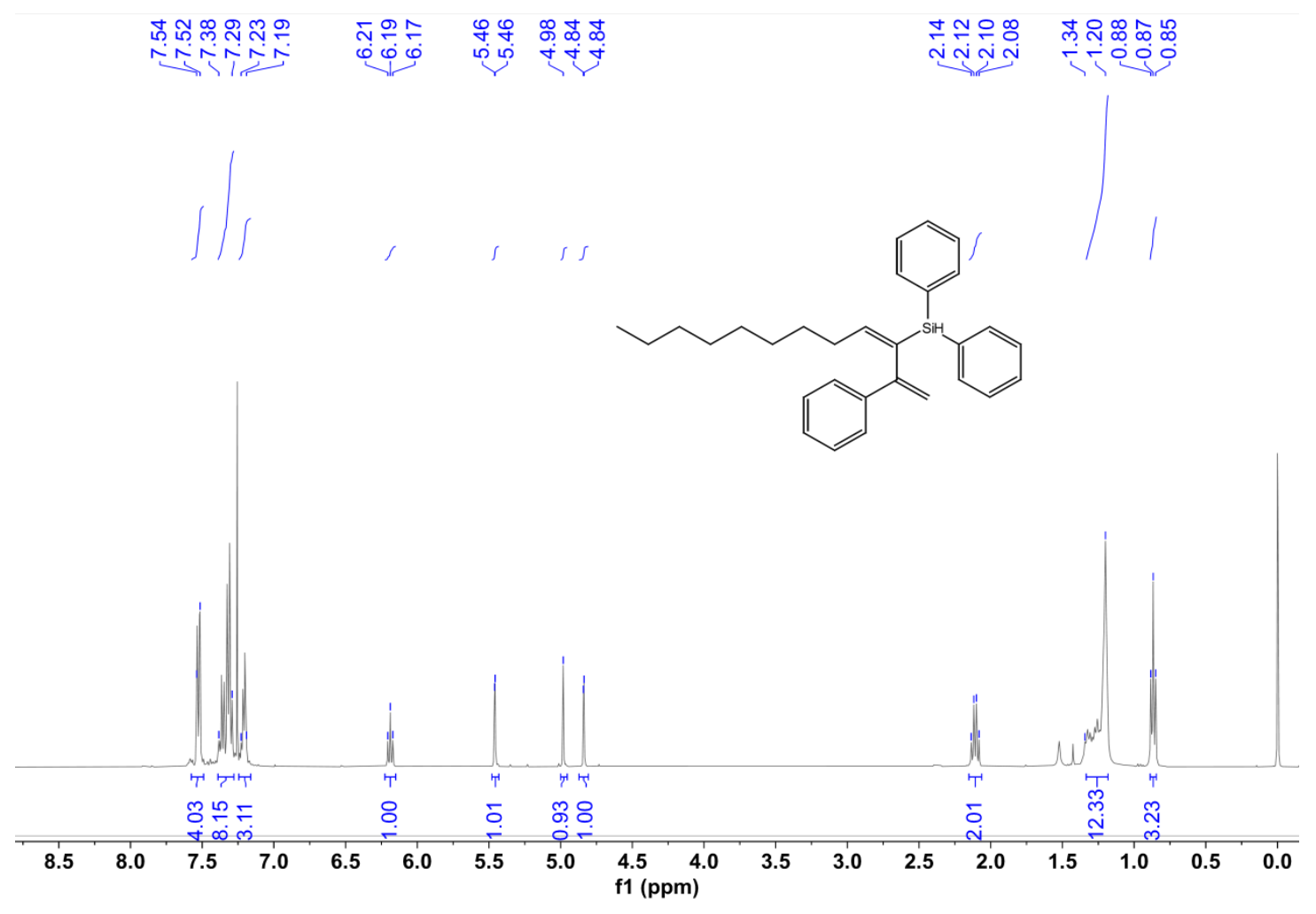

Figure $\mathrm{S}_{121}{ }^{1} \mathrm{H}$ NMR $\left(\mathrm{CDCl}_{3}, 400 \mathrm{M}\right)$ spectrum of 4ag' 


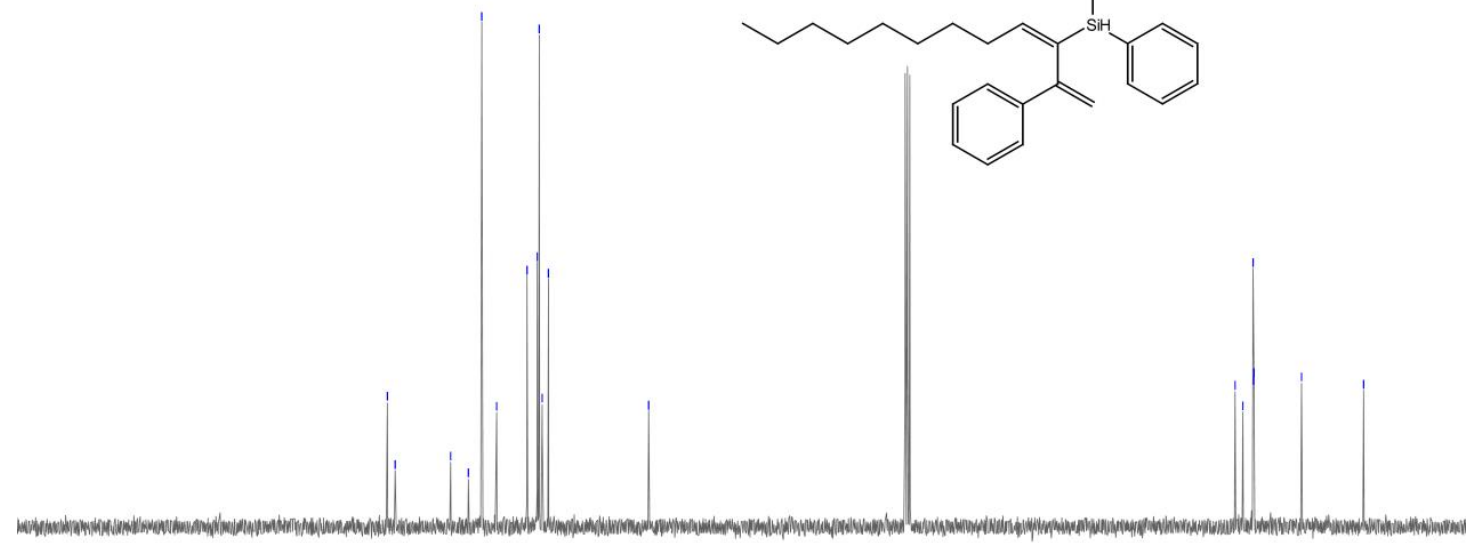

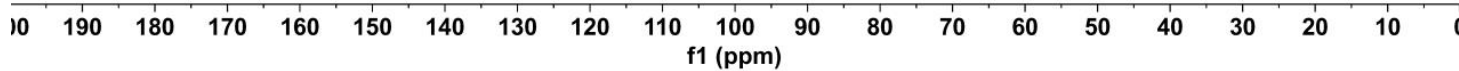

Figure $\mathrm{S122}^{13} \mathrm{C} \mathrm{NMR}\left(\mathrm{CDCl}_{3}, 100 \mathrm{M}\right)$ spectrum of 4ag'

\begin{tabular}{|c|c|c|}
\hline & 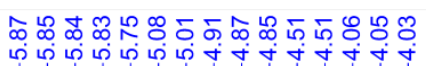 & Nָ Nָ \\
\hline
\end{tabular}

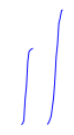

is 19<smiles>C=CCCCCCCCCC(=O)CC</smiles><smiles>Ic1ccccc1</smiles>

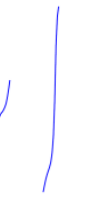<smiles>C=C(C)/C(=C/CCCCCCC)Nc1ccccc1</smiles>

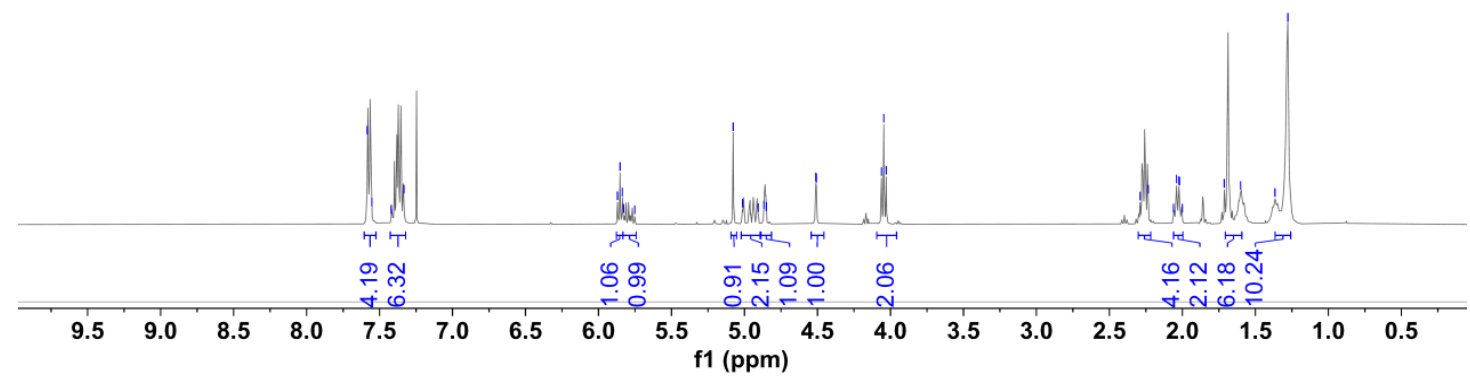

Figure $\mathrm{S123}^{1} \mathrm{H}$ NMR $\left(\mathrm{CDCl}_{3}, 400 \mathrm{M}\right)$ spectrum of 4ah' 


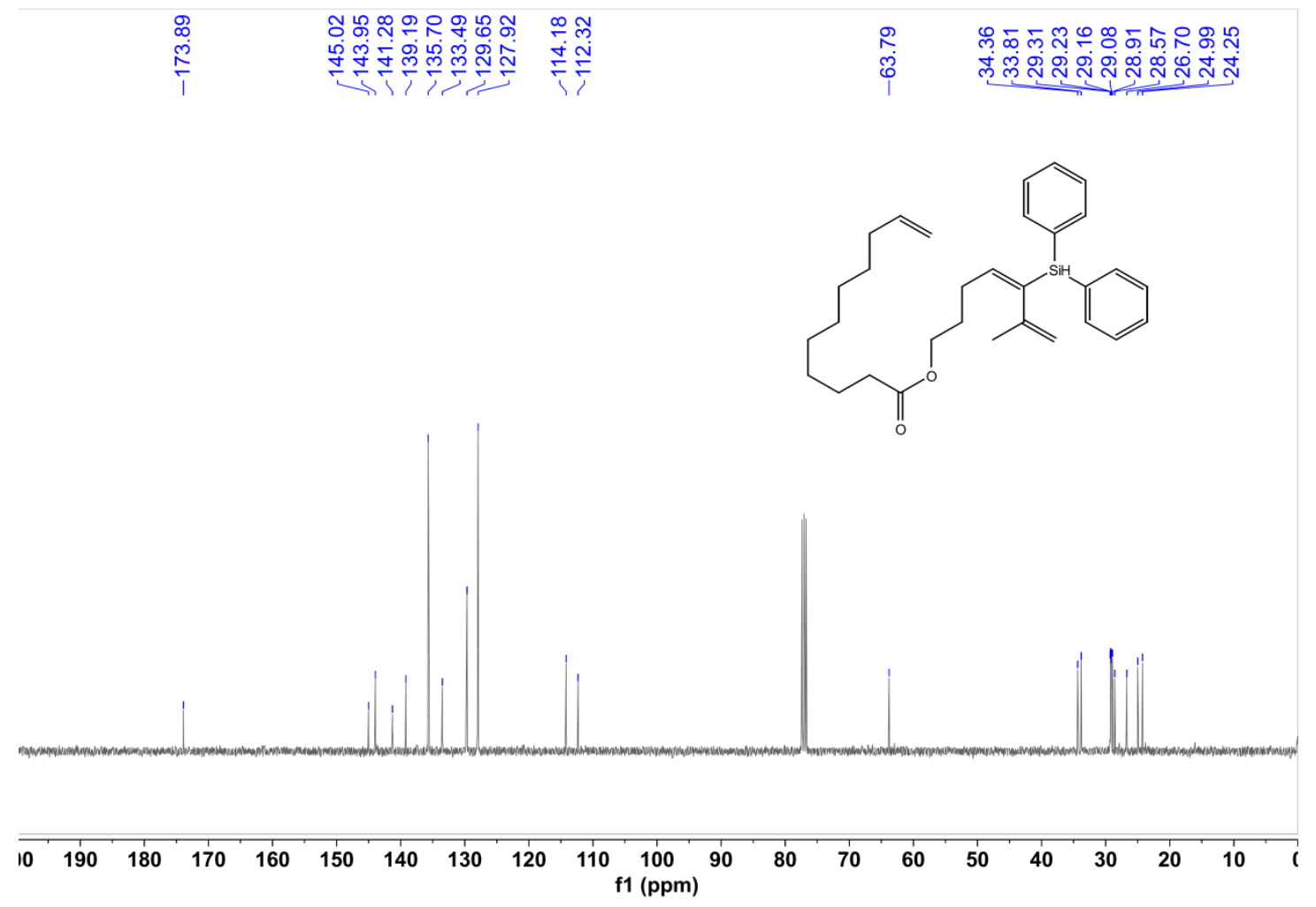

Figure $\mathrm{S}_{24}{ }^{13} \mathrm{C} \mathrm{NMR}\left(\mathrm{CDCl}_{3}, 100 \mathrm{M}\right)$ spectrum of 4ah'
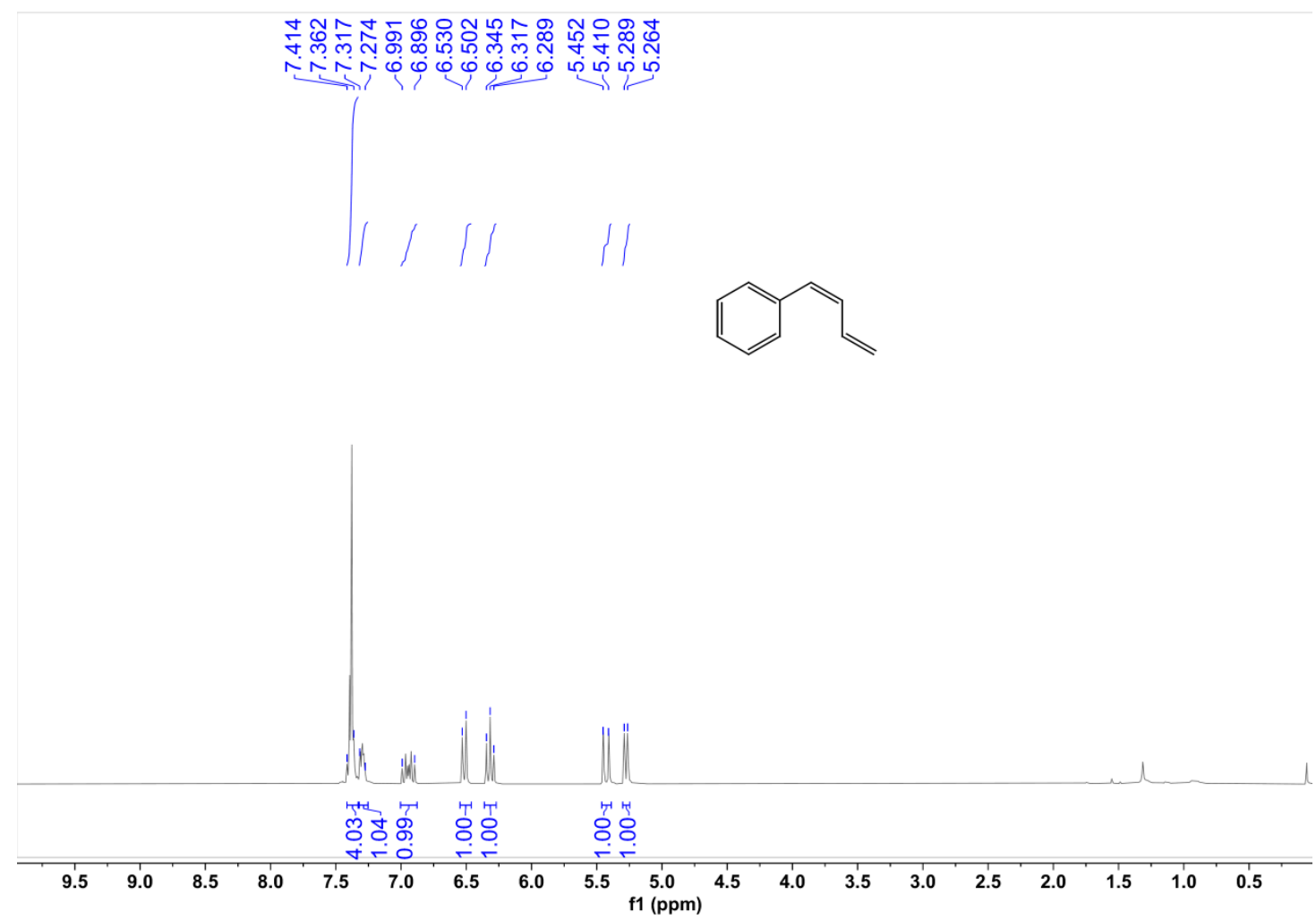

Figure $\mathrm{S125}^{1} \mathrm{H} \mathrm{NMR}\left(\mathrm{CDCl}_{3}, 400 \mathrm{M}\right)$ spectrum of $5 \mathrm{a}$ 


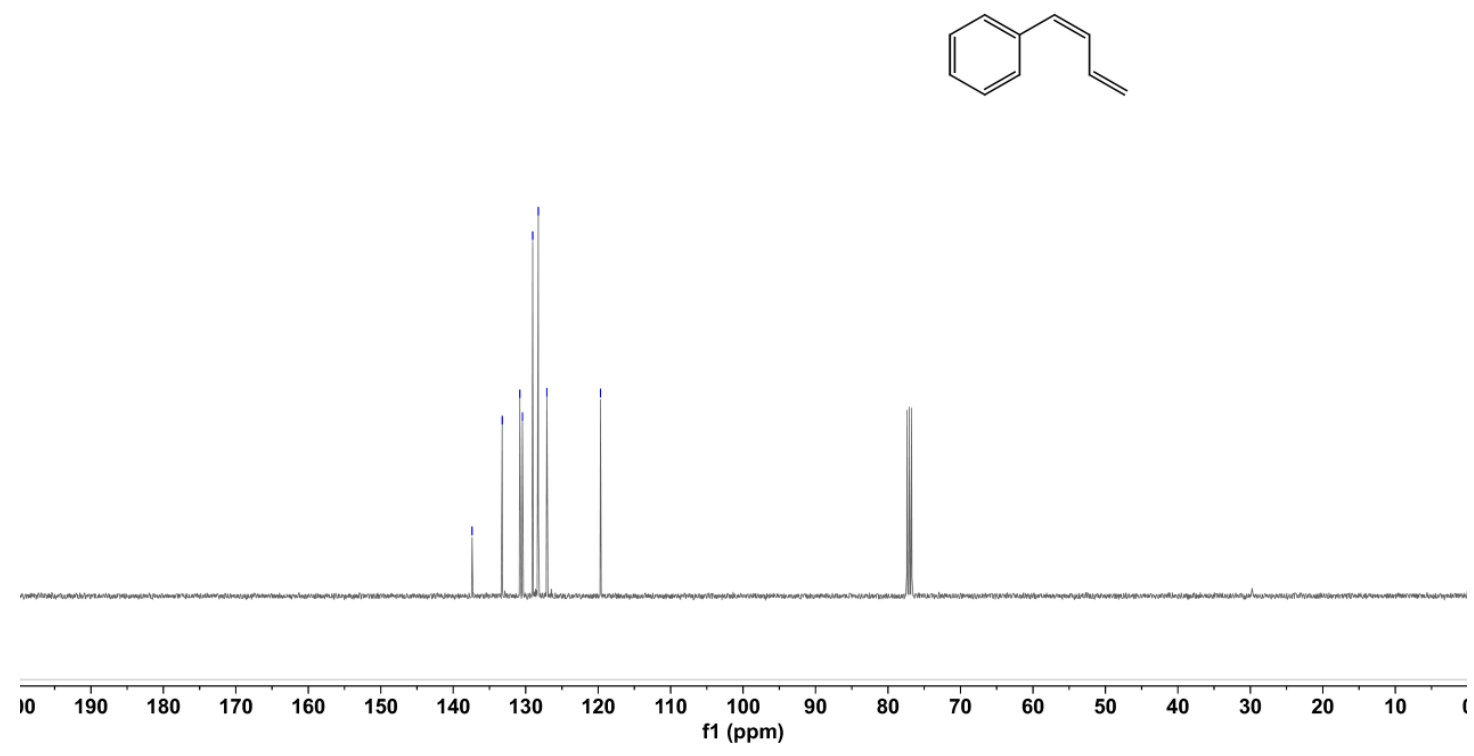

Figure $\mathrm{S126}^{13} \mathrm{C} \mathrm{NMR}\left(\mathrm{CDCl}_{3}, 100 \mathrm{M}\right)$ spectrum of 5a

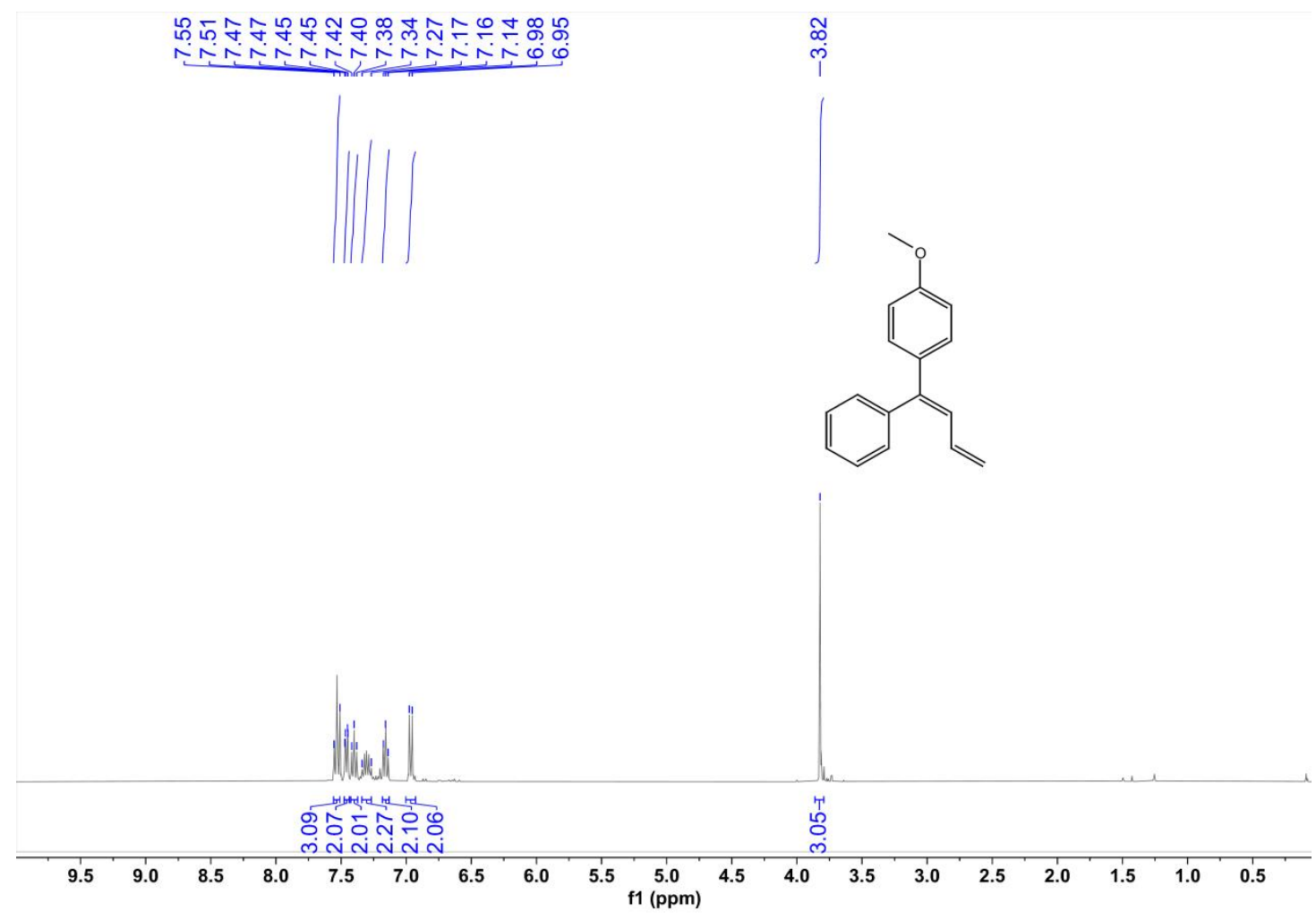

Figure $\mathrm{S127}{ }^{1} \mathrm{H} \mathrm{NMR}\left(\mathrm{CDCl}_{3}, 400 \mathrm{M}\right)$ spectrum of $6 \mathrm{a}$ 


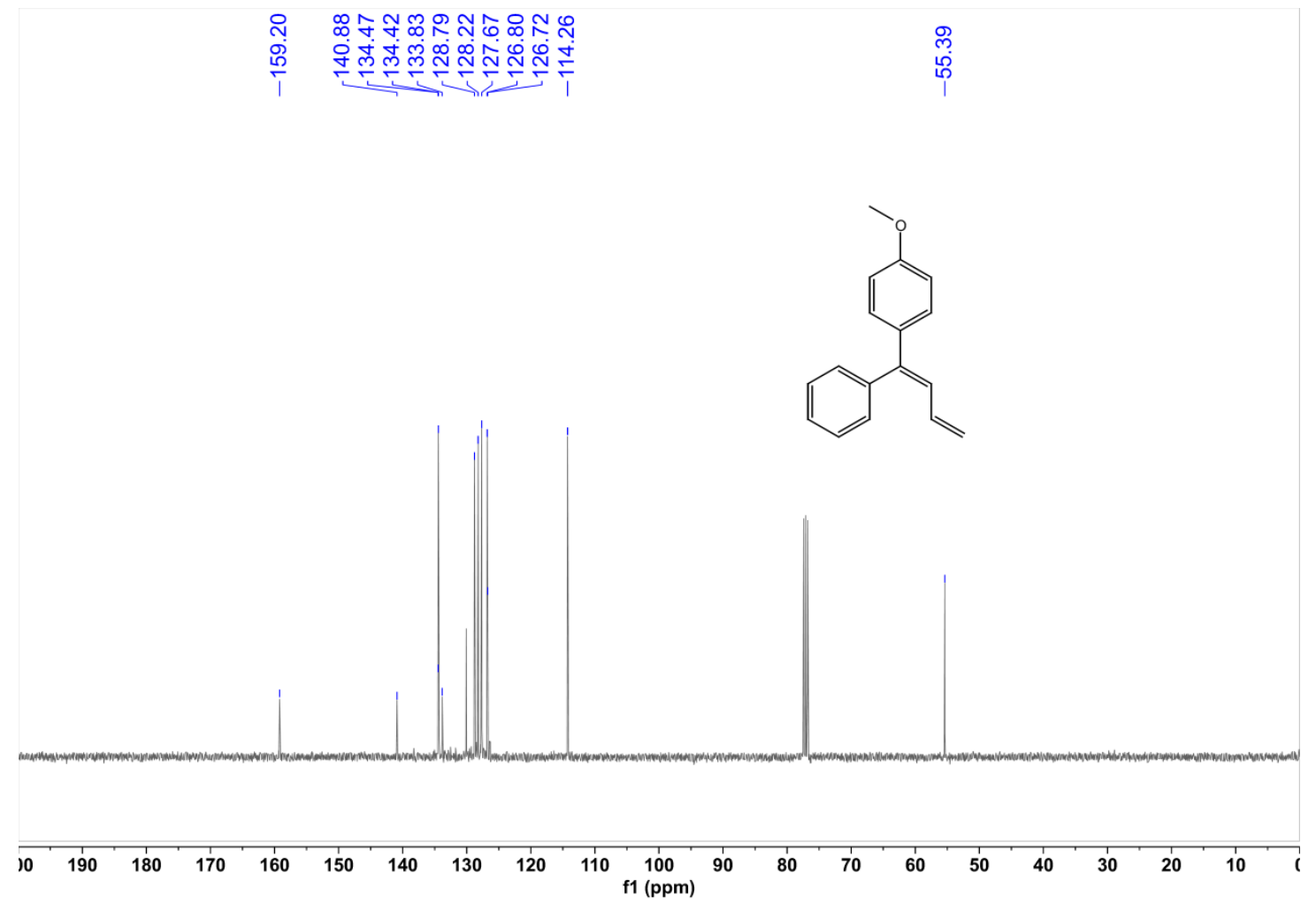

Figure $\mathrm{S128}^{13} \mathrm{C} \mathrm{NMR}\left(\mathrm{CDCl}_{3}, 100 \mathrm{M}\right)$ spectrum of $6 \mathrm{a}$

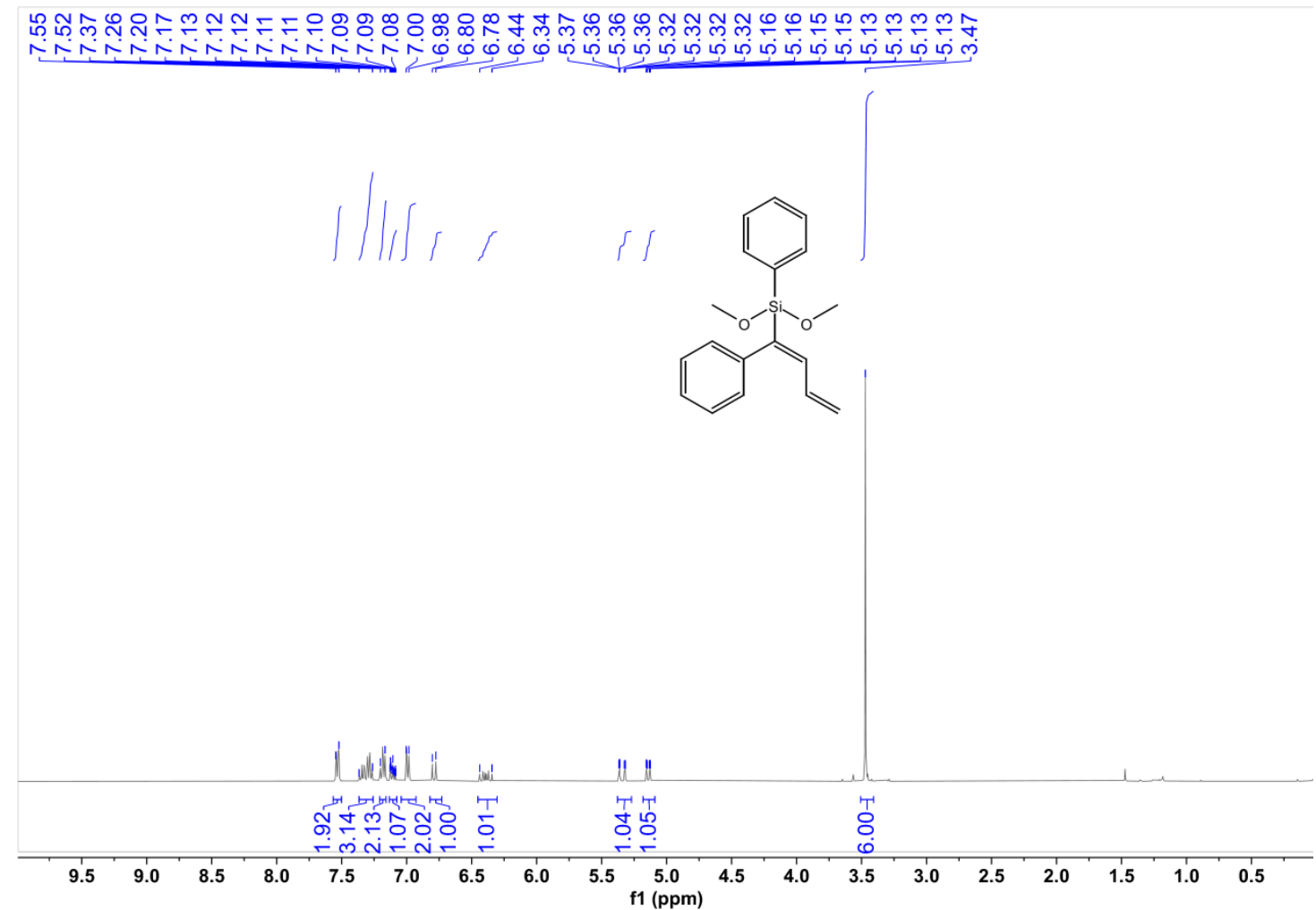

Figure $\mathrm{S129}^{1} \mathrm{H}$ NMR $\left(\mathrm{CDCl}_{3}, 400 \mathrm{M}\right)$ spectrum of $7 \mathrm{a}$ 


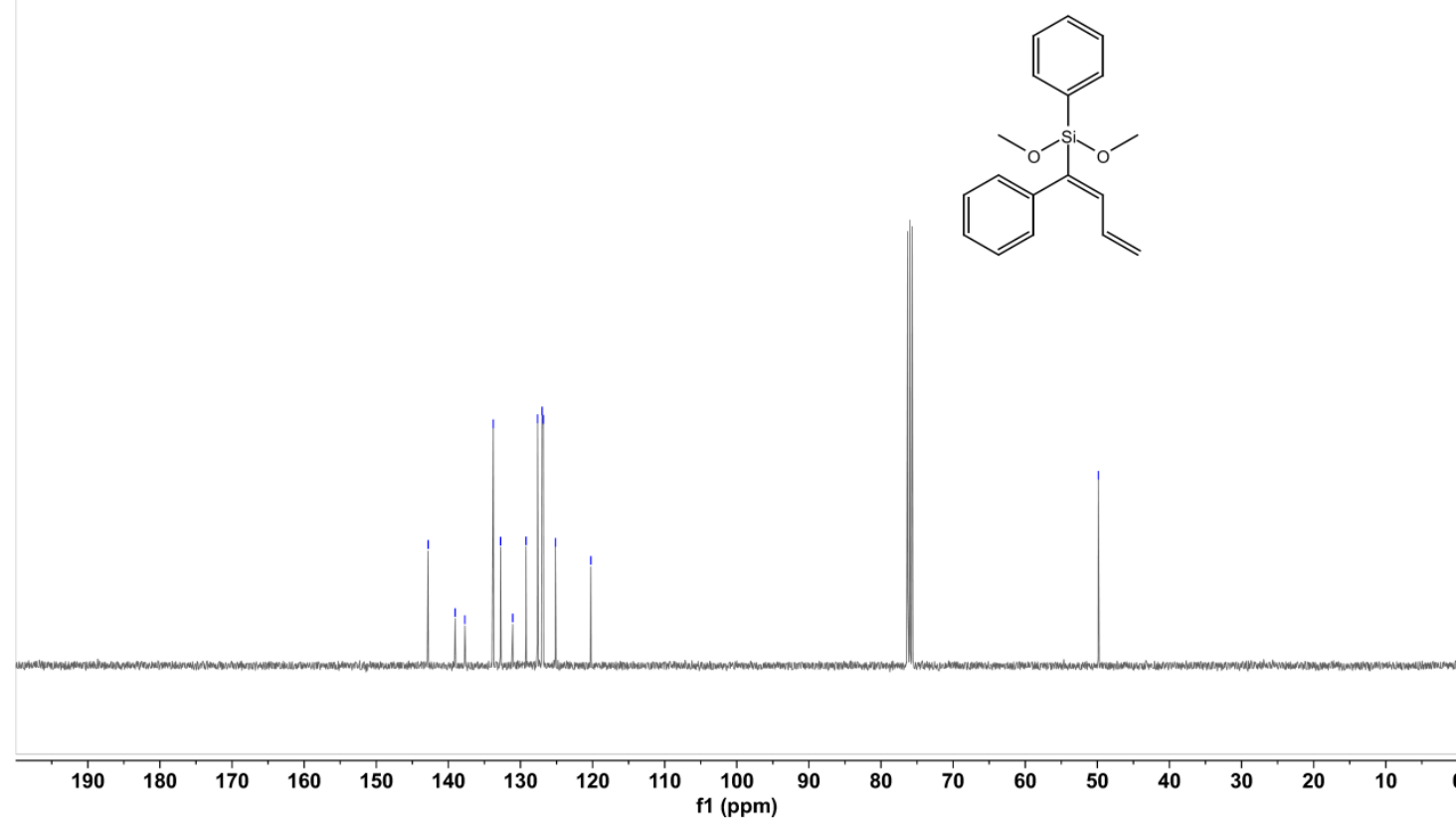

Figure S130 ${ }^{13} \mathrm{C} \mathrm{NMR}\left(\mathrm{CDCl}_{3}, 100 \mathrm{M}\right)$ spectrum of 7a 